Supporting Information for

\title{
Assessing Ligand and Counterion Effects in the Noble Metal Catalyzed Cycloisomerization Reactions of 1,6-Allenynes: a Combined Experimental and Theoretical Approach
}

Florian Jaroschik, ${ }^{\ddagger}$ Antoine Simonneau, ${ }^{\star}$ Gilles Lemière, ${ }^{\ddagger}$ Kevin Cariou, ${ }^{\ddagger}$ Nicolas Agenet, ${ }^{*}$ Hani Amouri, ${ }^{\star}$ Corinne Aubert, ${ }^{\ddagger}$ Jean-Philippe Goddard, ${ }^{\ddagger}$ Denis Lesage, ${ }^{\ddagger}$ Max Malacria, ${ }^{\star}$ Yves Gimbert, ${ }^{*}, \mathbb{I}$ Vincent Gandon ${ }^{*}$, and Louis Fensterbank ${ }^{*}, \$$

\footnotetext{
* Sorbonne Universités, UPMC Univ Paris 06 and CNRS, IPCM (UMR 8232), F-75005 Paris, France

${ }^{\S}$ Institut de Chimie Moléculaire et des Matériaux d'Orsay, CNRS UMR 8182, Univ. Paris-Sud, Université ParisSaclay, bâtiment 420, 91405 Orsay cedex (France)

"Univ Grenoble Alpes and CNRS, DCM (UMR 5250), F-38000 Grenoble, France

* louis.fensterbank@ upmc.fr, vincent.gandon@u-psud.fr, yves.gimbert@ ujf-grenoble.fr
}

\section{Table of Contents}

$\begin{array}{ll}\text { 1. General Experimental Conditions } & \text { S2 }\end{array}$

2. General Catalytic Procedures for Tables 1-4 S3

3. Synthesis and Characterization of Allenyne $9 \quad \mathrm{~S} 4$

4. Characterisation of Cycloisomerization Products $2,3,4,6,7,8,10,11,13,14,15$ S4

5. Experiments with Deuterated Allenynes 1-D and 1-D6 $\quad$ S11

6. Characterization of Product 18 and Synthesis of Gold Complexes 19 and $20 \quad$ S13

7. DFT calculations, including Figures S1-S6, Tables S1-S9 and Schemes S1-S2 S15

$\begin{array}{ll}\text { 8. References } & \text { S110 }\end{array}$

9. NMR Spectra $\quad$ S111 


\section{General Experimental Conditions}

THF and diethyl ether were distilled over sodium-benzophenone ketyl, toluene over sodium and dichloromethane over calcium hydride. $n$-Butyllithium was purchased as a $2.5 \mathrm{M}$ solution in hexanes. Gold complexes $\mathbf{C 1}$ and $\mathbf{C 5}$ were prepared according to literature procedures. ${ }^{1,2}$ Triphenylphosphine gold chloride, the complexes C2, C3, C4, C6, the ligand L1 and simple gold and silver salts were purchased. All commercially available chemicals were used as received. Allenynes 1, 1-D, 1-D 6 , 5 and 12 were prepared according to the literature. ${ }^{3,4}$ Thin layer chromatography (TLC) was performed on silica gel. $60 \AA$ Silica gel (35-70 mm) was used for column chromatography. NMR spectra $\left({ }^{1} \mathrm{H},{ }^{13} \mathrm{C},{ }^{31} \mathrm{P}\right.$, DEPT, COSY, HMQC, HMBC, NOE) were recorded at room temperature on 250, 400, 500 and $600 \mathrm{MHz}$ spectrometers. Chemical shifts are given in $\mathrm{ppm}$, referenced to the residual solvent resonance as internal standard $\left(\mathrm{CHCl}_{3} \delta=7.26\right.$ and $\mathrm{C}_{6} \mathrm{H}_{6} \delta=7.16$ for ${ }^{1} \mathrm{H}$ NMR; $\mathrm{CDCl}_{3} \delta=77.16$ and $\mathrm{C}_{6} \mathrm{H}_{6} \delta=$ 128.06 for ${ }^{13} \mathrm{C}$ NMR), or to the phosphorus resonance of $\mathrm{H}_{3} \mathrm{PO}_{4}(\delta=0.0 \mathrm{ppm})$ for ${ }^{31} \mathrm{P} \mathrm{NMR}$. Coupling constants $(\mathrm{J})$ are given in Hertz $(\mathrm{Hz})$. The terms $\mathrm{m}, \mathrm{s}, \mathrm{d}, \mathrm{t}$ and $\mathrm{q}$ refer to multiplet, singlet, doublet, triplet and quartet; br means that the signal is broad. When possible, ${ }^{1} \mathrm{H}$ and ${ }^{13} \mathrm{C}$ signals were assigned on the basis of DEPT and 2D-NMR (COSY, HMBC) experiments. Due to the gold quadrupole, ${ }^{13} \mathrm{C}$ NMR signals of carbon atoms of triple bonds attached to gold and in propargylic positions are very broad and are not observed. Low resolution mass spectra (MS) and high resolution mass spectra (HRMS) were measured by the Service de Spectrométrie de Masse de l'Université Pierre et Marie Curie, Paris VI. Infrared spectra (IR) were recorded on an ATR spectrometer. Absorbance frequencies are given in $\mathrm{cm}^{-1}$ at the peak maximum and at the medium $\mathrm{C} \equiv \mathrm{C}$ triple bond band.

All the ESI mass spectra were recorded using a Quattro II triple-quadrupole mass spectrometer equipped with a Z-spray source. The data were acquired using Masslynx software (version 4.0). The ESI capillary voltage was maintained at $3.5 \mathrm{kV}$ (positive mode), and the cone voltage $(\mathrm{Vc})$ was set at $20 \mathrm{~V}$. Nitrogen was used as the desolvation and nebulization gas. The source and desolvation temperatures were kept at $90{ }^{\circ} \mathrm{C}$ and $30^{\circ} \mathrm{C}$, respectively. The stock solutions $(1 \mu \mathrm{g} / \mu \mathrm{L})$ were made in $\mathrm{CH}_{2} \mathrm{Cl}_{2}$ and the working standard were prepared by further diluting the stock solution to achieve a final concentration of $40 \mu \mathrm{M}$ for the $\mathbf{C 1}$ catalyst, $800 \mu \mathrm{M}$ for substrate $\mathbf{1}$. $4 \%$ of $\mathrm{CH}_{3} \mathrm{OD}$ (or $\mathrm{CD}_{3} \mathrm{OD}$ ) was added to the $\mathbf{1}$ solution. The sample solutions were mixed in a microreactor ${ }^{5}$ using two infusion pumps operating at flow rates of $300 \mu \mathrm{L} / \mathrm{h}$ (for C1) and $120 \mu \mathrm{L} / \mathrm{h}$ (for 1) prior to the introduction into 
the ESI source of the mass spectrometer. The ESI mass spectra were recorded by scanning the first analyzer Q1.

\section{General Catalytic Procedures for Table 1, 2, 3 and 4}

All reactions were conducted under an atmosphere of argon.

\section{General procedure A :}

(L) $\mathrm{AuCl}(1 \mathrm{~mol} \%)$ was added to a solution of $\mathrm{AgX}(1 \mathrm{~mol} \%)$ in anhydrous $\mathrm{CH}_{2} \mathrm{Cl}_{2}(0.025 \mathrm{M})$. The mixture was stirred for $5 \mathrm{~min}$ and the reaction was cooled to the given temperature. Then the substrate was added and the reaction was followed by TLC. When the reaction was complete the mixture was filtered through a short pad of silica. The solvent was then evaporated under reduced pressure and purification by flash column chromatography over silica gel afforded the cycloisomerization products.

\section{General procedure B :}

The catalyst (x mol\%) was added to a solution of the substrate in anhydrous $\mathrm{CH}_{2} \mathrm{Cl}_{2}$, toluene or diethyl ether $(0.025 \mathrm{M})$ at the given temperature. The reaction was followed by TLC. When the reaction was complete the mixture was filtered through a short pad of silica. The solvent was then evaporated under reduced pressure and purification by flash column chromatography over silica gel afforded the cycloisomerization products.

\section{General procedure C:}

$\mathrm{AgX}(5 \mathrm{~mol} \%)$ and phosphine ligand $\mathbf{L}(5 \mathrm{~mol} \%)$ were placed in a round bottom flask and kept under an argon atmosphere. Then $\mathrm{CH}_{2} \mathrm{Cl}_{2}(0.025 \mathrm{M})$ was added at room temperature and the reaction was stirred for $30 \mathrm{~min}$ before adding the substrate. The reaction continued until complete disappearance of the starting material as indicated by TLC. The mixture was then filtered through a short pad of silica. The solvent was evaporated under reduced pressure and purification by flash column chromatography over silica gel afforded the cycloisomerization products. 


\section{Synthesis and Characterization of Allenyne 9}

$N$-(2,4-Dimethyl-penta-2,3-dienyl)-4-methyl- $N$-prop-2-y nyl-benzenesulfonamide (9)

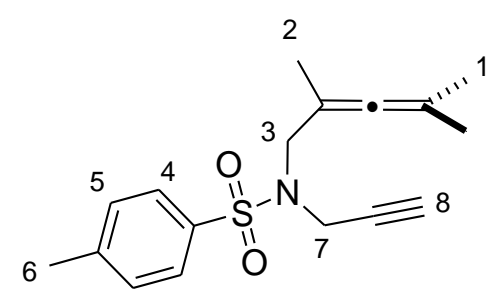

To a solution of 2,4-dimethyl-penta-2,3-dien-1-ol (448 mg, $4.0 \mathrm{mmol}, 1$ eq.) in THF (40 mL) at $0^{\circ} \mathrm{C}$ were successively added tosylpropargylamine (836 mg, $4.0 \mathrm{mmol}, 1$ eq.) and $\mathrm{PPh}_{3}$ (1.049 g, $4.0 \mathrm{mmol}, 1$ eq.). Then DIAD (0.792 mL, $4.0 \mathrm{mmol}, 1$ eq.) was added dropwise to this mixture over $45 \mathrm{~min}$. The reaction was stirred overnight and concentrated under reduced pressure. Purification by flash chromatography (PE/diethyl ether: 9/1) afforded the allene 9 (777 $\mathrm{mg}, 64 \%)$ as a colorless oil.

${ }^{1} \mathbf{H}$ NMR $\left(\mathbf{C D C l}_{3}, 250 \mathrm{MHz}\right) \delta 7.72\left(\mathrm{~d}, 2 \mathrm{H}, J=8.0 \mathrm{~Hz}, \mathrm{H}_{4}\right), 7.27\left(\mathrm{~d}, 2 \mathrm{H}, J=8.0 \mathrm{~Hz}, \mathrm{H}_{5}\right)$, $4.10\left(\mathrm{~d}, 2 \mathrm{H}, J=2.5 \mathrm{~Hz}, \mathrm{H}_{7}\right), 3.70\left(\mathrm{~s}, 2 \mathrm{H}, \mathrm{H}_{3}\right), 2.41\left(\mathrm{~s}, 3 \mathrm{H}, \mathrm{H}_{2}\right), 1.93\left(\mathrm{t}, 1 \mathrm{H}, J=2.5 \mathrm{~Hz}, \mathrm{H}_{5}\right)$, $1.65\left(\mathrm{~s}, 3 \mathrm{H}, \mathrm{H}_{2}\right), 1.64\left(\mathrm{~s}, 6 \mathrm{H}, \mathrm{H}_{1}\right)$.

${ }^{13}$ C NMR (CDCl 3 , 62.5 MHz) $\delta 201.6$ (C allene), $143.5(\mathrm{C}), 136.2(\mathrm{C}), 129.5(2 \mathrm{CH}), 127.9$ $(2 \mathrm{CH}), 95.6(\mathrm{C}), 91.3(\mathrm{C}), 76.5\left(\mathrm{C}_{\text {alkyne }}\right), 73.5\left(\mathrm{C}_{\text {alkyne}}\right), 50.9\left(\mathrm{CH}_{2}\right), 35.4\left(\mathrm{CH}_{2}\right), 21.7\left(\mathrm{CH}_{3}\right)$, $20.7\left(2 \mathrm{CH}_{3}\right), 16.6\left(\mathrm{CH}_{3}\right)$.

IR (neat) $3275,3031,2980,2909,2861,1598,1495,1445,1161 \mathrm{~cm}^{-1}$.

HRMS calculated for $\left[\mathrm{C}_{17} \mathrm{H}_{21} \mathrm{NNaO}_{2} \mathrm{~S}^{+}: 326.1190\right.$, found: 326.1195 .

\section{Characterisation of Cycloisomerization Products $2,3,4,6,7,8,10,11,13,14,15$}

5,5-Bis-methoxymethyl-1,7-dimethyl-2,4,5,6-tetrahydro-1H-indene $(2)^{3 \mathrm{a}}$

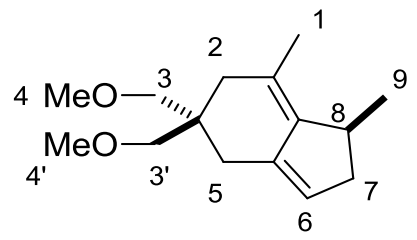

Colorless oil.

${ }^{1} \mathbf{H}$ NMR (400 MHz, CDCl $) \delta 5.41$ (bs, $\left.\mathrm{H}_{6}\right), 3.30$ (s, 3H, H4), 3.28 (s, 3H, H4), 3.19 (d, 2H, $\left.J=1.5 \mathrm{~Hz}, \mathrm{H}_{3}\right), 3.13\left(\mathrm{~s}, 2 \mathrm{H}, \mathrm{H}_{3}{ }^{\prime}\right), 2.83\left(\mathrm{qd}, 1 \mathrm{H}, J=7.1,6.6 \mathrm{~Hz}, \mathrm{H}_{8}\right), 2.64$ (dd, 1H, $J=16.8$, 
$\left.6.6 \mathrm{~Hz}, \mathrm{H}_{7}\right), 2.18\left(\mathrm{~s}, 2 \mathrm{H}, \mathrm{H}_{2}\right), 2.00\left(\mathrm{~d}, 1 \mathrm{H}, J=16.9 \mathrm{~Hz}, \mathrm{H}_{5}\right), 1.90\left(\mathrm{~d}, 1 \mathrm{H}, J=16.8 \mathrm{~Hz}, \mathrm{H}_{7}\right)$, $1.88\left(\mathrm{~d}, 1 \mathrm{H}, J=16.9 \mathrm{~Hz}, \mathrm{H}_{5}\right), 1.70\left(\mathrm{~s}, 3 \mathrm{H}, \mathrm{H}_{1}\right), 1.00\left(\mathrm{~d}, 3 \mathrm{H}, J=7.1 \mathrm{~Hz}, \mathrm{H}_{9}\right)$.

${ }^{1} \mathbf{H}$ NMR (400 MHz, C $\left.\mathbf{6}_{6} \mathbf{D}_{6}\right) \delta .46(\mathrm{~s}, 1 \mathrm{H}), 3.28(\mathrm{~s}, 2 \mathrm{H}), 3.24(\mathrm{~d}, J=3.6 \mathrm{~Hz}, 2 \mathrm{H}), 3.15(\mathrm{~s}$, $3 \mathrm{H}), 3.12(\mathrm{~s}, 3 \mathrm{H}), 2.82$ (q, $J=6.9 \mathrm{~Hz}, 1 \mathrm{H}), 2.66(\mathrm{dd}, J=16.8,5.8 \mathrm{~Hz}, 1 \mathrm{H}), 2.50-2.35$ (m, 2H), $2.17(\mathrm{~d}, J=17.2 \mathrm{~Hz}, 1 \mathrm{H}), 2.06(\mathrm{~d}, J=17.3 \mathrm{~Hz}, 1 \mathrm{H}), 1.92$ (d, $J=16.8 \mathrm{~Hz}, 1 \mathrm{H}), 1.70$ (s, $3 \mathrm{H}), 1.07(\mathrm{~d}, J=7.0 \mathrm{~Hz}, 3 \mathrm{H})$.

${ }^{13}$ C NMR (100 MHz, CDCl $) \delta 143.3(\mathrm{C}), 138.5(\mathrm{C}), 125.2(\mathrm{CH}), 120.3(\mathrm{C}), 76.5\left(\mathrm{CH}_{2}\right)$, $75.9\left(\mathrm{CH}_{2}\right), 59.6\left(\mathrm{CH}_{3}\right), 59.5\left(\mathrm{CH}_{3}\right), 40.1\left(\mathrm{CH}_{2}\right), 39.5(\mathrm{C}), 36.0\left(\mathrm{CH}_{2}\right), 33.2(\mathrm{CH}), 29.5\left(\mathrm{CH}_{2}\right)$, $21.7\left(\mathrm{CH}_{3}\right), 19.4\left(\mathrm{CH}_{3}\right)$.

IR (neat) 2953, 2918, 2888, 2846, 1476, 1446, 1116, $1105 \mathrm{~cm}^{-1}$.

Elemental Analysis for $\mathrm{C}_{15} \mathrm{H}_{24} \mathrm{O}_{2}$ : C 76.23, H 10.24. Found C 75.95, H 10.46.

2-Isopropenyl-5,5-bis-methoxymethyl-1-methyl-3-methylene-cyclohexene $(3)^{3 \mathrm{~b}}$

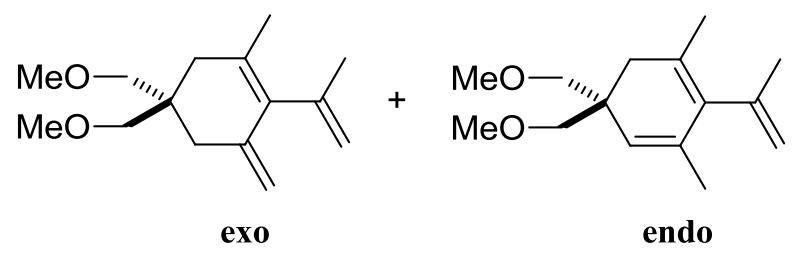

Colorless oil.

${ }^{1}$ H NMR (400 MHz, $\left.\mathbf{C D C l}_{3}\right) \delta 5.22$ (s, 1H, =CH, endo), 5.10-5.09 (m, 1H, $\mathrm{CH}_{3} \mathrm{C}=\mathrm{CH}$, exo), 5.06-5.05 (m, 1H, $\mathrm{CH}_{3} \mathrm{C}=\mathrm{CH}$, endo), 4.83 (s, 1H, =CHH, exo), 4.73 (s, 1H, =CHH, exo), 4.63-4.62 (m, 1H, $\mathrm{CH}_{3} \mathrm{C}=\mathrm{CH} H$, exo), 4.60-4.59 (m, $1 \mathrm{H}, \mathrm{CH}_{3} \mathrm{C}=\mathrm{CH} H$, endo), 3.32 (s, 6H, $\mathrm{MeO}$, exo), 3.31 (s, 6H, $\mathrm{MeO}$, endo), 3.24 (d, $2 \mathrm{H}, J=8.6 \mathrm{~Hz}, \mathrm{CH}_{2} \mathrm{O}$, endo), 3.20 (s, 4H, $\mathrm{CH}_{2} \mathrm{O}$, exo), 3.19 (d, $2 \mathrm{H}, J=8.6 \mathrm{~Hz}, \mathrm{CH}_{2} \mathrm{O}$, endo), 2.25 (s, 2H, $\mathrm{CH}_{2}$, exo), 2.08 (s, $2 \mathrm{H}, \mathrm{CH}_{2}$, endo), 2.02 (s, 2H, $\mathrm{CH}_{2}$, exo), 1.78 (s, 3H, $\mathrm{CH}_{3}$, exo), 1.76 (s, 3H, $\mathrm{CH}_{3}$, endo), 1.72 (s, 3H, $\mathrm{CH}_{3}$, endo), 1.70 (d, $3 \mathrm{H}, J=1.2 \mathrm{~Hz}, \mathrm{CH}_{3}$, endo), 1.69 (s, $3 \mathrm{H}, \mathrm{CH}_{3}$, exo).

${ }^{13}$ C NMR (100 MHz, CDCl $) \delta 144.1$ (C), 143.8 (C), 140.8 (C), 136.0 (C), 134.5 (C), 134.0 (C), 129.7 (C), 127.4 (C), 122.8 (=CH, endo), $114.9\left(\mathrm{CH}_{3} \mathrm{C}=\mathrm{CH} 2\right.$, exo), $114.6\left(\mathrm{CH}_{3} \mathrm{C}=\mathrm{CH}_{2}\right.$, endo), 110.4 (s, $1 \mathrm{H},=\mathrm{CH}_{2}$, exo), 76.0 (2C, $\mathrm{CH}_{2} \mathrm{O}$, exo), $75.2\left(2 \mathrm{C}, \mathrm{CH}_{2} \mathrm{O}\right.$, endo), $59.5(2 \mathrm{C}+2 \mathrm{C}$, $\mathrm{MeO}$, exo+ endo), 40.3 (C, exo), 38.7 (C, endo), $36.6\left(\mathrm{CH}_{2}\right.$, exo), $36.4\left(\mathrm{CH}_{2}\right.$, exo $), 34.3\left(\mathrm{CH}_{2}\right.$, endo), $24.1\left(\mathrm{CH}_{3}\right.$, endo), $23.2\left(\mathrm{CH}_{3}\right.$, exo), $21.0\left(\mathrm{CH}_{3}\right), 20.5\left(\mathrm{CH}_{3}\right), 20.4\left(\mathrm{CH}_{3}\right)$.

IR (neat) $3077,2978,2919,2886,2824,1644,1625,1606,1447,1376, \mathrm{~cm}^{-1}$. 


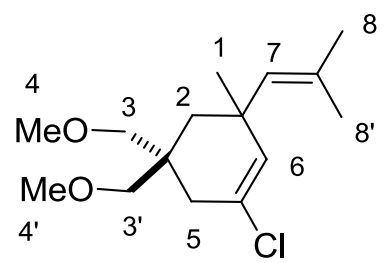

Colorless oil.

${ }^{1}$ H NMR (400 MHz, CDCl $) \delta 5.85\left(\mathrm{~s}, 1 \mathrm{H}, \mathrm{H}_{6}\right), 5.14\left(\mathrm{~m}, 1 \mathrm{H}, \mathrm{H}_{7}\right), 3.31\left(\mathrm{~s}, 3 \mathrm{H}, \mathrm{H}_{4}\right), 3.23(\mathrm{~s}$, $3 \mathrm{H}, \mathrm{H}_{4}$ ) $, 3.22\left(\mathrm{~d}, 2 \mathrm{H}, J=3.0 \mathrm{~Hz}, \mathrm{H}_{3}\right), 3.19\left(\mathrm{~d}, 2 \mathrm{H}, J=4.0 \mathrm{~Hz}, \mathrm{H}_{3}, 2.23(\mathrm{dd}, 1 \mathrm{H}, J=14.0\right.$, $\left.2.0 \mathrm{~Hz}, \mathrm{H}_{5}\right), 2.07$ (d, 1H, $\left.J=14.0 \mathrm{~Hz}, \mathrm{H}_{5}\right), 1.77$ (d, $\left.1 \mathrm{H}, J=13.6 \mathrm{~Hz}, \mathrm{H}_{2}\right), 1.69$ (d, 3H, $J=1.2$ $\left.\mathrm{Hz}, \mathrm{H}_{8}\right), 1.65$ (d, 3H, $J=1.2 \mathrm{~Hz}, \mathrm{H}_{8}$ ), 1.32 (d, 1H, $\left.J=13.6 \mathrm{~Hz}, \mathrm{H}_{2}\right), 1.12$ (s, 3H, $\mathrm{H}_{1}$ ).

${ }^{13}$ C NMR (100 MHz, CDCl $) \delta 133.4(\mathrm{CH}), 132.0(\mathrm{CH}), 130.0(\mathrm{C}), 128.7(\mathrm{C}), 77.5\left(\mathrm{CH}_{2}\right)$, $74.6\left(\mathrm{CH}_{2}\right), 59.3\left(\mathrm{CH}_{3}\right), 58.8\left(\mathrm{CH}_{3}\right), 40.8\left(\mathrm{CH}_{2}\right), 39.5(\mathrm{C}), 38.5\left(\mathrm{CH}_{2}\right), 37.5(\mathrm{C}), 30.9\left(\mathrm{CH}_{3}\right)$, $28.0\left(\mathrm{CH}_{3}\right), 19.0\left(\mathrm{CH}_{3}\right)$.

IR (neat) 2960, 2922, 2889, 1703, 1656, 1476, 1371, 1175, 1100.

HRMS calculated for $\left[\mathrm{C}_{15} \mathrm{H}_{25} \mathrm{CINaO}_{2}\right]^{+}: 295.1435$, found 295.1437 .

Dimethyl 1,2-dihydro-1,7-dimethyl-4H-indene-5,5(6H)-dicarboxylate $(6)^{3 a}$<smiles>COC(C)[C@@]1(C(C)=O)CC2=CC[C@@H](C)C2=C(C)C1</smiles>

Colorless oil.

${ }^{1}$ H NMR (300 MHz, CDCl $) \delta 5.49$ (s, 1H), 3.67 (s, 3H), 3.67 (s, 3H), $2.91-2.72$ (m, 3H), $2.71-2.51(\mathrm{~m}, 1 \mathrm{H}), 2.56(\mathrm{~s}, 2 \mathrm{H}), 1.91(\mathrm{~d}, J=17.0 \mathrm{~Hz}, 1 \mathrm{H}), 1.73(\mathrm{~s}, 3 \mathrm{H}), 0.95$ (d, $J=7.0 \mathrm{~Hz}$, $3 \mathrm{H})$.

${ }^{13}$ C NMR (75 MHz, CDCl $) \delta 171.9$ (C), 171.8 (C), 144.1 (C), 136.0 (C), 126.0 (CH), 120.2 (C), $54.9(\mathrm{C}), 52.8\left(\mathrm{CH}_{3}\right), 52.6\left(\mathrm{CH}_{3}\right), 40.2\left(\mathrm{CH}_{2}\right), 36.8\left(\mathrm{CH}_{2}\right), 33.1(\mathrm{CH}), 31.1\left(\mathrm{CH}_{2}\right), 21.4$ $\left(\mathrm{CH}_{3}\right), 19.1\left(\mathrm{CH}_{3}\right)$.

IR (neat) 2954, 2908, 2847, 1733, 1435, 1245, 1174, $1045 \mathrm{~cm}^{-1}$.

HRMS calculated for $\left[\mathrm{C}_{15} \mathrm{H}_{20} \mathrm{NaO}_{4}\right]^{+}: 287.1259$, found: 287.1250 . 
Dimethyl 3-methyl-5-methylene-4-(prop-1-en-2-yl)cyclohex-3-ene-1,1-dicarboxylate (7)

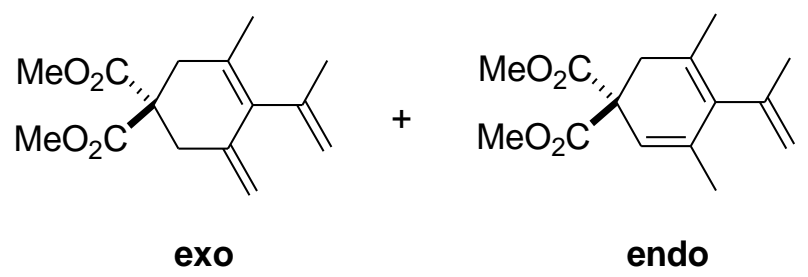

Colorless oil.

${ }^{1}$ H NMR (500 MHz, $\mathbf{C D C l}_{3}$ ) $\delta 5.59$ (s, 1H, =CH, endo), 5.08 (s, 1H, $\mathrm{CH}_{3} \mathrm{C}=\mathrm{CHH}$, exo), 5.06 (s, $1 \mathrm{H}, \mathrm{CH}_{3} \mathrm{C}=\mathrm{CHH}$, endo), $4.86(\mathrm{~s}, 1 \mathrm{H},=\mathrm{CHH}$, exo), $4.80(\mathrm{~s}, 1 \mathrm{H},=\mathrm{CH} H$, exo), $4.59(\mathrm{~s}, 1 \mathrm{H}$, $\mathrm{CH}_{3} \mathrm{C}=\mathrm{CH} H$, exo), 4.59 (s, $1 \mathrm{H}, \mathrm{CH}_{3} \mathrm{C}=\mathrm{CH} H$, endo), 3.70 (s, 6H, $\mathrm{MeO}$, endo), 3.69 (s, 6H, MeO, endo), 2.84 (s, 2H, $\mathrm{CH}_{2}$, exo), 2.67 (s, 2H, $\mathrm{CH}_{2}$, endo), 2.62 (s, $2 \mathrm{H}, \mathrm{CH}_{2}$, exo), 1.76 (s, $3 \mathrm{H}, \mathrm{CH}_{3}$, endo), 1.74 (d, $J=1.5 \mathrm{~Hz}, 3 \mathrm{H}, \mathrm{CH}_{3}$, endo), 1.73 (s, 6H, $2 \mathrm{CH}_{3}$, exo), 1.71 (s, 3H, $\mathrm{CH}_{3}$, endo).

${ }^{13}$ C NMR (125 MHz, CDCl 3 ) $\delta 171.6$ (C, endo), 171.4 (C, exo), 143.0 (C, endo), 142.9 (C, exo), 138.7 (C, exo), 136.3 (C, exo), 135.5 (C, endo), 134.7 (C, endo), 129.3 (C, exo), 127.8 (C, endo), 116.3 (=CH, endo), $115.3\left(\mathrm{CH}_{3} \mathrm{C}=\mathrm{CH}_{2}\right.$, exo), $115.2\left(\mathrm{CH}_{3} \mathrm{C}=\mathrm{CH}_{2}\right.$, endo), 111.4 (= $\mathrm{CH}_{2}$, exo), $54.7\left(\mathrm{C}\right.$, endo), $54.3(\mathrm{C}$, exo $), 52.8\left(2 \mathrm{CH}_{3}\right.$, endo $), 52.7\left(2 \mathrm{CH}_{3}\right.$, exo $), 37.0\left(2 \mathrm{CH}_{2}\right.$, exo), $35.7\left(\mathrm{CH}_{2}\right.$, endo), $23.8\left(\mathrm{CH}_{3}\right.$, endo), $23.0\left(\mathrm{CH}_{3}\right.$, exo), $20.7\left(\mathrm{CH}_{3}\right.$, exo $), 20.2\left(\mathrm{CH}_{3}\right.$, endo), $20.1\left(\mathrm{CH}_{3}\right.$, endo).

IR (neat) 3075, 2953, 2914, 2851, 1737, 1435, 1251, 1202, 1178, 1090, 1073, $898 \mathrm{~cm}^{-1}$.

HRMS calculated for $\left[\mathrm{C}_{15} \mathrm{H}_{20} \mathrm{NaO}_{4}\right]^{+}: 287.1259$, found: 287.1248 .

\section{Dimethyl 3-chloro-5-methyl-5-(2-methylprop-1-enyl)cyclohex-3-ene-1,1-dicarboxylate} (8)

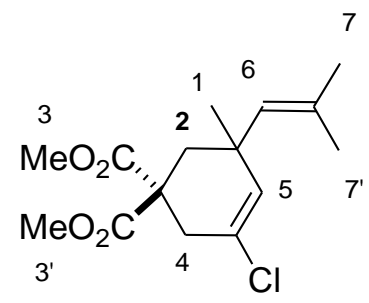

Colorless oil.

${ }^{1}$ H NMR (600 MHz, CDCl M $_{3} 5.79\left(\mathrm{~s}, 1 \mathrm{H}, \mathrm{H}_{5}\right), 4.92\left(\mathrm{~s}, 1 \mathrm{H}, \mathrm{H}_{6}\right), 3.71\left(\mathrm{~s}, 3 \mathrm{H}, \mathrm{H}_{3}\right), 3.62(\mathrm{~s}$, $3 \mathrm{H}, \mathrm{H}_{3}$ ) $) 2.89\left(\mathrm{~d}, 1 \mathrm{H}, J=17.4 \mathrm{~Hz}, \mathrm{H}_{4}\right), 2.56\left(\mathrm{~d}, 1 \mathrm{H}, J=17.4 \mathrm{~Hz}, \mathrm{H}_{4}\right), 2.38(\mathrm{~d}, 1 \mathrm{H}, J=14.4$

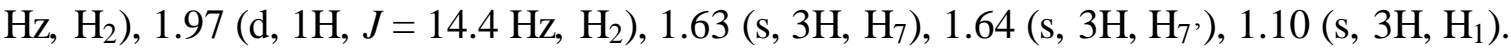


${ }^{13}$ C NMR (150 MHz, CDCl $) \delta 171.6(\mathrm{C}), 170.6(\mathrm{C}), 132.5(\mathrm{C}), 132.3(\mathrm{CH}), 130.8(\mathrm{CH})$, $126.9(\mathrm{C}), 53.4(\mathrm{C}), 52.9\left(\mathrm{CH}_{3}\right), 52.6\left(\mathrm{CH}_{3}\right), 40.0\left(\mathrm{CH}_{2}\right), 38.5(\mathrm{C}), 36.8\left(\mathrm{CH}_{2}\right), 30.2\left(\mathrm{CH}_{3}\right)$, $28.2\left(\mathrm{CH}_{3}\right), 18.7\left(\mathrm{CH}_{3}\right)$.

IR (neat) 2956, 2928, 2865, 1739, 1435, 1321, 1245, 1208, $1167 \mathrm{~cm}^{-1}$.

HRMS calculated for $\left[\mathrm{C}_{15} \mathrm{H}_{21} \mathrm{CINaO}_{4}\right]^{+}: 323.1026$, found 323.1024 .

2,3,5,6-tetrahydro-4,5-dimethyl-2-tosyl-1 H-cyclopenta[c]py ridine

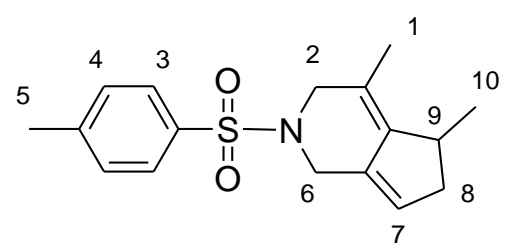

Colorless oil.

${ }^{1}$ H NMR $\left(\mathbf{C D C l}_{3}, 250 \mathrm{MHz}\right) \delta 7.63\left(\mathrm{~d}, 2 \mathrm{H}, \mathrm{J}=8.4 \mathrm{~Hz}, \mathrm{H}_{3}\right), 7.18\left(\mathrm{~d}, 2 \mathrm{H}, \mathrm{J}=8.4 \mathrm{~Hz}, \mathrm{H}_{4}\right), 5.49$ (s, 1H), $3.98\left(\mathrm{~d}, 1 \mathrm{H}, J=16 \mathrm{~Hz}, \mathrm{H}_{6}\right), 3.90\left(\mathrm{~d}, 1 \mathrm{H}, J=16 \mathrm{~Hz}, \mathrm{H}_{6}\right), 3.61\left(\mathrm{~d}, 2 \mathrm{H}, J=4.2 \mathrm{~Hz}, \mathrm{H}_{2}\right)$, $2.69\left(\mathrm{q}, 1 \mathrm{H}, J=6.6 \mathrm{~Hz}, \mathrm{H}_{9}\right), 2.55\left(\mathrm{dm}, 1 \mathrm{H}, J=18 \mathrm{~Hz}, \mathrm{H}_{8}\right), 2.39$ (s, 3H, H$)_{5}, 1.88$ (d, 1H, $J=$ $\left.18 \mathrm{~Hz}, \mathrm{H}_{8}\right), 1.64\left(\mathrm{~s}, 3 \mathrm{H}, \mathrm{H}_{1}\right), 0.86\left(\mathrm{~d}, 3 \mathrm{H}, J=6.6 \mathrm{~Hz}, \mathrm{H}_{10}\right)$

${ }^{13}$ C NMR (CDCl 3 , 75 MHz) $\delta 143.4(\mathrm{C}), 143.2$ (C), 134.3 (C), 134.1 (C), $129.4\left(\mathrm{CH}_{2}\right), 127.8$ $\left(\mathrm{CH}_{2}\right), 125.8(\mathrm{CH}), 118.6(\mathrm{C}), 49.0\left(\mathrm{CH}_{2}\right), 44.6\left(\mathrm{CH}_{2}\right), 40.2\left(\mathrm{CH}_{2}\right), 33.0(\mathrm{CH}), 21.6\left(\mathrm{CH}_{3}\right)$, $20.9\left(\mathrm{CH}_{3}\right), 16.6\left(\mathrm{CH}_{3}\right)$.

HRMS calculated for $\left[\mathrm{C}_{17} \mathrm{H}_{21} \mathrm{NNaO}_{2} \mathrm{~S}\right]^{+}: 326.1190$, found: 326.1195 .

1,2,3,6-tetrahydro-5-methyl-3-methylene-4-(prop-1-en-2-yl)-1-tosylpy ridine (11)

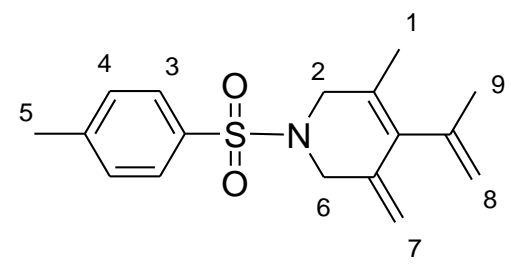

${ }^{1} \mathbf{H}$ NMR $\left(\mathbf{C D C l}_{\mathbf{3}}, \mathbf{4 0 0} \mathbf{M H z}\right) \delta 7.66\left(\mathrm{~d}, 2 \mathrm{H}, \mathrm{J}=8.4 \mathrm{~Hz}, \mathrm{H}_{3}\right), 7.21\left(\mathrm{~m}, 2 \mathrm{H}, \mathrm{J}=8.4 \mathrm{~Hz}, \mathrm{H}_{4}\right)$, $5.05\left(\mathrm{~m}, 1 \mathrm{H}, \mathrm{H}_{7}\right), 4.86\left(\mathrm{~s}, 1 \mathrm{H}, \mathrm{H}_{8}\right), 4.80\left(\mathrm{~s}, 1 \mathrm{H}, \mathrm{H}_{8}\right), 4.44\left(\mathrm{~m}, 1 \mathrm{H}, \mathrm{H}_{7}\right), 3.86\left(\mathrm{~s}, 2 \mathrm{H}, \mathrm{H}_{2}\right), 3.69$ (s, 2H, $\left.\mathrm{H}_{6}\right), 2.40\left(\mathrm{~s}, 3 \mathrm{H}, \mathrm{H}_{5}\right), 1.63\left(\mathrm{~m}, 3 \mathrm{H}, \mathrm{H}_{9}\right), 1.62\left(\mathrm{~s}, 3 \mathrm{H}, \mathrm{H}_{1}\right)$.

${ }^{13}$ C NMR (CDCl 3 , 100 MHz) $\delta 143.6$ (C), 141.3 (C), 136.3 (C), 135.6 (C), 134.0 (C), 129.6 $(\mathrm{CH}), 127.9(\mathrm{CH}), 127.4(\mathrm{C}), 115.9\left(\mathrm{CH}_{2}\right), 110.9\left(\mathrm{CH}_{2}\right), 49.5\left(\mathrm{CH}_{2}\right), 22.7\left(\mathrm{CH}_{3}\right), 21.6\left(\mathrm{CH}_{3}\right)$, $17.8\left(\mathrm{CH}_{3}\right)$. 
IR (neat) 3063, 2979, 2919, 1681, 1597, 1449, 1350, 1155, 1105, 1025.

HRMS calculated for $\left[\mathrm{C}_{17} \mathrm{H}_{22} \mathrm{NO}_{2} \mathrm{~S}\right]^{+}: 304.1371$, found: 304.1371 .

Diethyl-1,2-dihydro-1-methy l-4H-indene-5,5(6H)-dicarboxy late (13)

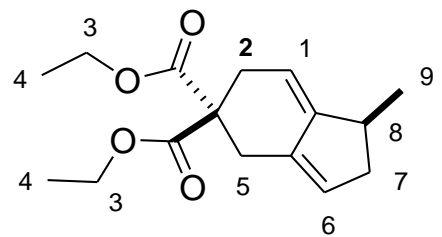

colorless oil.

${ }^{1}$ H NMR (CDCl 3 , 500 MHz) 5.62 (bs, 1H, H $\mathrm{H}_{6}$ ), 5.30 (bs, 1H, $\left.\mathrm{H}_{1}\right), 4.15$ (m, 4H, $\mathrm{H}_{3}$ ), 2.87 (bs, 2H, $\left.\mathrm{H}_{2}\right), 2.69\left(\mathrm{~m}, 3 \mathrm{H}, \mathrm{H}_{5}+\mathrm{H}_{8}\right), 2.65\left(\mathrm{dd}, 1 \mathrm{H}, J=18.0,6.0 \mathrm{~Hz}, \mathrm{H}_{7}\right), 1.97(\mathrm{~d}, 1 \mathrm{H}, J=18 \mathrm{~Hz}$, $\left.\mathrm{H}_{7}\right), 1.21\left(\mathrm{t}, 6 \mathrm{H}, J=7.5 \mathrm{~Hz}, \mathrm{H}_{4}\right), 1.04(\mathrm{~d}, 3 \mathrm{H}, J=7.0 \mathrm{~Hz})$.

${ }^{13} \mathbf{C ~ N M R}\left(\mathbf{C D C l}_{3}, 125\right.$ MHz) : 171.5 (C), 171.3 (C), 150.8 (C), 137.1 (C), 128.5 (CH), 110.8 $(\mathrm{CH}), 61.4\left(\mathrm{CH}_{2}\right), 61.4\left(\mathrm{CH}_{2}\right), 54.4(\mathrm{C}), 40.3\left(\mathrm{CH}_{2}\right), 34.4(\mathrm{CH}), 31.25\left(\mathrm{CH}_{2}\right), 31.21\left(\mathrm{CH}_{2}\right)$, $21.3\left(\mathrm{CH}_{3}\right), 14.2\left(\mathrm{CH}_{3}\right), 14.1\left(\mathrm{CH}_{3}\right)$.

IR (neat) 2979, 2959, 2709, 2846, 1732, 1463, 1444, 1366, 1304, 1249, 1180, $1062 \mathrm{~cm}^{-1}$. HRMS calculated for $\left[\mathrm{C}_{16} \mathrm{H}_{22} \mathrm{NaO}_{4}\right]^{+}: 301.1416$, found : 301.1412

Diethyl 5-methylene-4-(prop-1-en-2-yl)cyclohex-3-ene-1,1-dicarboxylate $(14)^{4}$

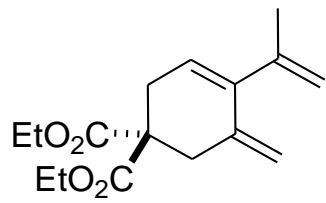

colorless oil.

${ }^{1}$ H NMR $\left(\mathbf{C D C l}_{3}, 400 \mathrm{MHz}\right) \delta 5.62(\mathrm{t}, 1 \mathrm{H}, J=4.6 \mathrm{~Hz}), 5.01(\mathrm{~s}, 1 \mathrm{H}), 4.98(\mathrm{~s}, 1 \mathrm{H}), 4.97$ (s, 1H), $4.83(\mathrm{~s}, 1 \mathrm{H}), 4.21-4.13(\mathrm{~m}, 4 \mathrm{H}), 2.87(\mathrm{bs}, 2 \mathrm{H}), 2.70(\mathrm{~d}, 2 \mathrm{H}, J=4.4 \mathrm{~Hz}), 1.84(\mathrm{~s}, 3 \mathrm{H})$, $1.22(\mathrm{t}, 6 \mathrm{H}, J=7.0 \mathrm{~Hz})$.

${ }^{13}$ C NMR (C6 $\mathbf{D}_{6} 100$ MHz) $\delta 171.0$ (2CO), 144.4 (C), 141.7 (C), $138.1(\mathrm{C}), 123.3(\mathrm{CH})$, 114.6 $\left(\mathrm{CH}_{2}\right), 114.0\left(\mathrm{CH}_{2}\right), 61.6\left(2 \mathrm{CH}_{2}\right), 54.3(\mathrm{C}), 37.4\left(\mathrm{CH}_{2}\right), 31.6\left(\mathrm{CH}_{2}\right), 23.1\left(\mathrm{CH}_{3}\right), 14.2$ $\left(2 \mathrm{CH}_{3}\right)$.

IR (neat) 2975, 2924, 2830, 1732, $1467 \mathrm{~cm}^{-1}$. 
Diethyl 3-chloro-5-(2-methylprop-1-enyl)cyclohex-3-ene-1,1-dicarboxylate (15)

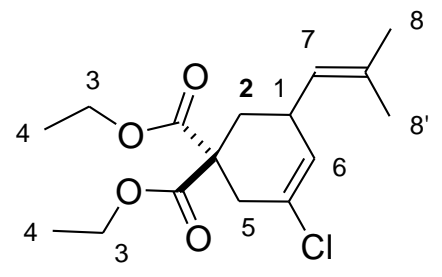

colorless oil.

${ }^{1} \mathbf{H}$ NMR (500 MHz, $\left.\mathbf{C D C l}_{3}\right) \delta 5.58\left(\mathrm{~m}, 1 \mathrm{H}, \mathrm{H}_{6}\right), 4.90\left(\mathrm{~d}, 1 \mathrm{H}, \mathrm{J}=9.0 \mathrm{~Hz}, \mathrm{H}_{7}\right), 4.15-4.22(\mathrm{~m}$, 4H, $\mathrm{H}_{3}$ ), 3.13 (br m, 1H, $\mathrm{H}_{1}$ ), 2.97 (d, 1H, $J=17.5 \mathrm{~Hz}, \mathrm{H}_{2}$ ), 2.67 (ddd, 1H, $J=17.5,4.0,2.5$ $\mathrm{Hz}, \mathrm{H}_{2}$ ), 2.34 (ddm, 1H, $J=13.0,4.0 \mathrm{~Hz}, \mathrm{H}_{5}$ ), 1.68 (s, 3H, $\left.\mathrm{H}_{8}\right), 1.62$ (s, 3H, $\mathrm{H}_{8}$ ), 1.58 (dd, $\left.1 \mathrm{H}, J=13.0,2.5 \mathrm{~Hz}, \mathrm{H}_{5}\right), 1.26\left(\mathrm{t}, 3 \mathrm{H}, \mathrm{J}=7.0 \mathrm{~Hz}, \mathrm{H}_{4}\right), 1.24\left(\mathrm{t}, 3 \mathrm{H}, \mathrm{J}=7.0 \mathrm{~Hz}, \mathrm{H}_{4}\right)$.

${ }^{13}$ C NMR (100 MHz, CDCl $) \delta 170.1(\mathrm{C}), 133.8(\mathrm{C}), 128.7(\mathrm{C}), 127.7(\mathrm{CH}), 125.9(\mathrm{CH})$, $61.7\left(\mathrm{CH}_{2}\right), 54.7(\mathrm{C}), 37.0\left(\mathrm{CH}_{2}\right), 33.8(\mathrm{CH}), 33.4\left(\mathrm{CH}_{2}\right), 25.7\left(\mathrm{CH}_{3}\right), 17.9\left(\mathrm{CH}_{3}\right), 14.2\left(\mathrm{CH}_{3}\right)$. IR (neat) 2979, 2934, 2910, 2874, 1736, 1445, 1367, 1296, 1245, $1179 \mathrm{~cm}^{-1}$. HRMS calculated for $\left[\mathrm{C}_{16} \mathrm{H}_{23} \mathrm{ClNaO}_{4}\right]^{+}: 337.1183$, found 337.1175 . 


\section{Experiments with Deuterated Allenynes 1-D and 1-D 6}

Allenyne 1-D

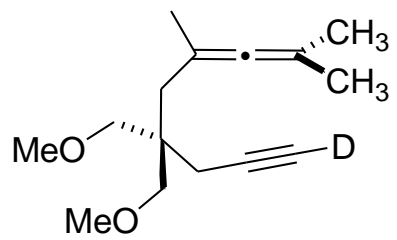

Colorless oil.

${ }^{1}$ H NMR (400 MHz, C $\left.6 \mathbf{D}_{6}\right) \delta 3.44(\mathrm{~s}, 4 \mathrm{H}), 3.15$ (s, 6H), 2.55 (s, 2H), 2.34 (s, 2H), 1.78 (s, $3 \mathrm{H}), 1.64(\mathrm{~s}, 6 \mathrm{H})$.

${ }^{13}$ C NMR (100 MHz, $\left.\mathbf{C D C l}_{3}\right) \delta$ 202.1, 142.6, 128.6, 75.8, 58.8, 43.1, 31.7, 31.0, 28.0, 21.8, 20.4, 13.8. Acetylenic CD could not be detected due to a too high signal/noise ratio.

IR (neat) $3300,2940,1540,1360,1190,1100 \mathrm{~cm}^{-1}$.

HRMS calculated for $\left[\mathrm{C}_{15} \mathrm{H}_{23} \mathrm{DNaO}_{2}\right]^{+}: 260.1731$, found: 260.1730 .

\section{Hydrindiene 2-D}

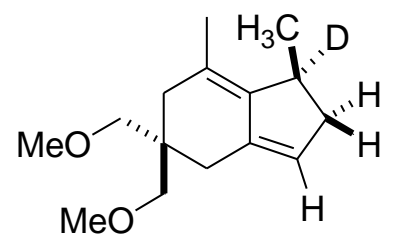

Colorless oil.

${ }^{1}$ H NMR (400 MHz, C $\left.\mathbf{6}_{6} \mathbf{D}_{6}\right) \delta 5.47(\mathrm{~s}, 1 \mathrm{H}), 3.29(\mathrm{~s}, 2 \mathrm{H}), 3.25(\mathrm{~d}, J=3.7 \mathrm{~Hz}, 2 \mathrm{H}), 3.15$ (s, $3 \mathrm{H}), 3.12(\mathrm{~s}, 3 \mathrm{H}), 2.66(\mathrm{~d}, J=16.9 \mathrm{~Hz}, 1 \mathrm{H}), 2.50-2.36(\mathrm{~m}, 2 \mathrm{H}), 2.18(\mathrm{~d}, J=17.2 \mathrm{~Hz}, 1 \mathrm{H})$, 2.07 (d, $J=17.2 \mathrm{~Hz}, 1 \mathrm{H}), 1.95-1.86(\mathrm{~m}, 1 \mathrm{H}), 1.70$ (s, 3H), 1.07 (s, 3H).

${ }^{13}$ C NMR (100 MHz, C $\left.\mathbf{C}_{6} \mathbf{D}_{6}\right) \delta 143.6(\mathrm{C}), 139.3(\mathrm{C}), 125.3(=\mathrm{CH}), 120.7(\mathrm{C}), 76.9\left(\mathrm{CH}_{2}\right), 75.8$ $\left(\mathrm{CH}_{2}\right), 59.1\left(\mathrm{CH}_{3}\right), 59.1\left(\mathrm{CH}_{3}\right), 40.4\left(\mathrm{CH}_{2}\right), 40.0(\mathrm{C}), 36.4\left(\mathrm{CH}_{2}\right), 33.2\left(\mathrm{t}, J_{\mathrm{C}-\mathrm{D}}=20.0 \mathrm{~Hz}, \mathrm{CD}\right)$, $30.0\left(\mathrm{CH}_{2}\right), 21.8\left(\mathrm{CH}_{3}\right), 19.5\left(\mathrm{CH}_{3}\right)$.

IR (neat) 2953, 2918, 2888, 2846, 1476, 1446, 1116, $1105 \mathrm{~cm}^{-1}$.

HRMS calculated for $\left[\mathrm{C}_{15} \mathrm{H}_{23} \mathrm{DNaO}_{2}\right]^{+}: 260.1731$, found: 260.1732 .

\section{Allenyne 1-D 6}

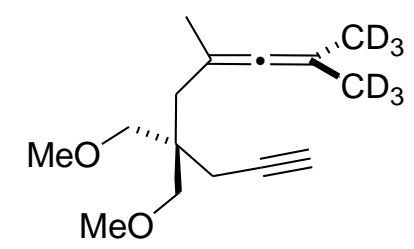

Colorless oil. 
${ }^{1}$ H NMR (400 MHz, C $\left.6 \mathbf{D}_{6}\right) \delta 3.44(\mathrm{~s}, 4 \mathrm{H}), 3.15(\mathrm{~s}, 6 \mathrm{H}), 2.56(\mathrm{~d}, J=2.7 \mathrm{~Hz}, 2 \mathrm{H}), 2.34(\mathrm{~s}$, 2H), $1.78(\mathrm{~s}, 3 \mathrm{H}), 1.77$ (t, $J=2.7 \mathrm{~Hz}, 1 \mathrm{H})$.

${ }^{13}$ C NMR (100 MHz, $\left.\mathbf{C D C l}_{3}\right) \delta 201.2$ ( $\left.\mathrm{C}_{\text {allene }}\right), 92.5$ (2 $\left.\mathrm{C}_{\text {allene }}\right), 81.8\left(\mathrm{C}_{\text {alkyne }}\right), 74.3\left(2 \mathrm{CH}_{2} \mathrm{O}\right)$, $70.0\left(\mathrm{C}_{\text {alkyne }}\right), 59.3\left(2 \mathrm{OCH}_{3}\right), 42.4(\mathrm{C}), 35.6\left(\mathrm{CH}_{2}\right), 22.3-22.1$ (m, overlapping with methyl and methylene signals, 2CD 3$), 22.3\left(\mathrm{CH}_{2}\right), 22.1\left(\mathrm{CH}_{3}\right)$.

IR (neat) $3305,1538,1364,1184,1110 \mathrm{~cm}^{-1}$.

HRMS calculated for $\left[\mathrm{C}_{15} \mathrm{H}_{18} \mathrm{D}_{6} \mathrm{NaO}_{2}\right]^{+}: 265.2045$, found: 265.2049 .

\section{Hydrindiene 2-D 6}

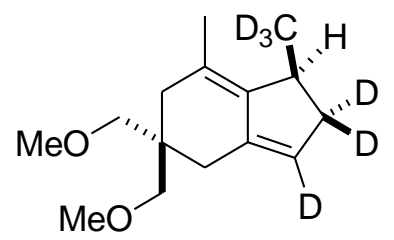

Colorless oil.

${ }^{1} \mathbf{H}$ NMR (300 MHz, C $\left.\mathbf{6}_{6} \mathbf{D}_{6}\right) \delta 3.29$ (s, 2H), 3.25 (d, $\left.J=2.1 \mathrm{~Hz}, 2 \mathrm{H}\right), 3.15$ (s, 3H), 3.12 (s, $3 \mathrm{H}), 2.79(\mathrm{~s}, 1 \mathrm{H}), 2.52-2.33(\mathrm{~m}, 2 \mathrm{H}), 2.13(\mathrm{q}, J=17.2 \mathrm{~Hz}, 2 \mathrm{H}), 1.72-1.69(\mathrm{~m}, 3 \mathrm{H})$.

${ }^{13}$ C NMR (100 MHz, C $\left.\mathbf{C}_{6}\right) \delta 143.7$ (C), 139.4 (C), 124.9 (t, $\left.J_{\mathrm{C}-\mathrm{D}}=24.3 \mathrm{~Hz},=\mathrm{CD}\right), 120.6$ (C), $76.9\left(\mathrm{CH}_{2}\right), 75.9\left(\mathrm{CH}_{2}\right), 59.1\left(\mathrm{CH}_{3}\right), 59.1\left(\mathrm{CH}_{3}\right), 40.0(\mathrm{C}), 39.5\left(\mathrm{t}, J_{\mathrm{C}-\mathrm{D}}=19.3 \mathrm{~Hz}, \mathrm{CD}_{2}\right)$, $36.4\left(\mathrm{CH}_{2}\right), 33.2(\mathrm{CH}), 30.0\left(\mathrm{CH}_{2}\right), 21.0$ (quint, $\left.J_{\mathrm{C}-\mathrm{D}}=19.0 \mathrm{~Hz}, \mathrm{CD}_{3}\right), 19.5\left(\mathrm{CH}_{3}\right)$.

IR (neat) 2953, 2918, 2888, 2846, 1476, 1446, 1116, $1105 \mathrm{~cm}^{-1}$.

HRMS calculated for $\left[\mathrm{C}_{15} \mathrm{H}_{18} \mathrm{D}_{6} \mathrm{NaO}_{2}\right]^{+}: 265.2045$, found: 265.2048 . 


\section{Characterization of Product 18 and Synthesis of Gold Complexes 19 and 20}

\section{4,4-bis(methoxymethyl)-2,8,8-trimethylbicyclo[4.2.0]octa-1,6-diene (18)}

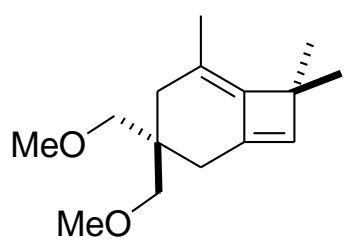

Colorless oil.

${ }^{1} \mathbf{H}$ NMR (400 MHz, $\left.\mathbf{C D C l}_{3}\right) \delta 6.00(\mathrm{~s}, 1 \mathrm{H}, \mathrm{C}=\mathrm{CH}), 3.31\left(\mathrm{~s}, 6 \mathrm{H}, \mathrm{OCH}_{3}\right), 3.20(\mathrm{~s}, 4 \mathrm{H}$, $\mathrm{CH}_{2} \mathrm{OMe}$ ), $2.05\left(\mathrm{~d}, J=1.2 \mathrm{~Hz}, 2 \mathrm{H}, \mathrm{CH}_{2} \mathrm{C}=\mathrm{CH}\right), 1.92\left(\mathrm{~s}, 2 \mathrm{H}, \mathrm{CH}_{2} \mathrm{MeC}=\mathrm{C}\right), 1.59$ (s, $3 \mathrm{H}$, $\left.\mathrm{CH}_{3} \mathrm{C}=\mathrm{C}\right), 1.27\left(\mathrm{~s}, 6 \mathrm{H}, \mathrm{CH}_{3}\right)$.

${ }^{13}$ C NMR (100 MHz, $\left.\mathbf{C D C l}_{3}\right) \delta 143.7(\mathrm{C}), 143.0(\mathrm{C}), 136.7(\mathrm{CH}), 111.2(\mathrm{C}), 76.5\left(2 \mathrm{CH}_{2}\right)$, $59.6\left(2 \mathrm{CH}_{3}\right), 50.9(\mathrm{C}), 41.7(\mathrm{C}), 34.6\left(\mathrm{CH}_{2}\right), 26.9\left(\mathrm{CH}_{2}\right), 25.2\left(2 \mathrm{CH}_{3}\right), 17.5\left(\mathrm{CH}_{3}\right)$.

HRMS calculated for $\left[\mathrm{C}_{15} \mathrm{H}_{24} \mathrm{NaO}_{2}\right]^{+}: 259.1669$, found: 259.1667 .

\section{Gold Complex 19}

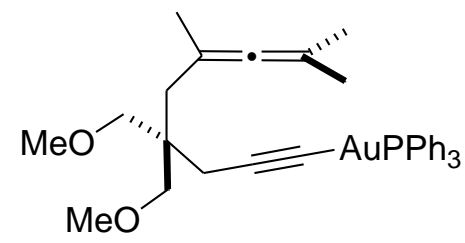

In an oven-dried Schlenk flask purged with argon was dissolved allenyne 1 (0.90 mmol, 0.21 g, 3.0 equiv) in THF $(12 \mathrm{~mL})$. The solution was cooled to $0{ }^{\circ} \mathrm{C}$ then $n$-BuLi $(0.38 \mathrm{~mL}$ of a $2.25 \mathrm{M}$ solution in hexanes, $0.90 \mathrm{mmol}, 3.0$ equiv) was added. A brown color could appear. The mixture was stirred at $0{ }^{\circ} \mathrm{C}$ for $10 \mathrm{~min}$, then $\mathrm{AuClPPh}_{3}(0.15 \mathrm{~g}, 0.30 \mathrm{mmol}, 1.0$ equiv) was added in one portion under an argon counterflow. The reaction was warmed to room temperature and allowed to stir for 3 hours. After that time, the reaction was quenched with a saturated aqueous solution of $\mathrm{NH}_{4} \mathrm{Cl}(6 \mathrm{~mL})$. The organic layer was removed under reduced pressure, then $\mathrm{CH}_{2} \mathrm{Cl}_{2}(12 \mathrm{~mL})$ was added. The aqueous layer was further extracted with $\mathrm{CH}_{2} \mathrm{Cl}_{2}$ and the combined organic extracts were dried over $\mathrm{MgSO}_{4}$. After removal of the solvent, a gum was obtained that can be purified in several manners: direct precipitation in $\mathrm{CHCl}_{3} /$ hexane followed by filtration on fritted glass and washing with hexane or slow precipitation in a 5:95 $\mathrm{CHCl}_{3} /$ hexane mixture at $-25{ }^{\circ} \mathrm{C}$ when the direct precipitation was not effective. Complex 19 was obtained as a white solid in $55 \%$ yield. 
${ }^{1} \mathbf{H}$ NMR $\left(\mathbf{C D C l}_{3}\right) \delta=7.55-7.41(\mathrm{~m}, 15 \mathrm{H}), 3.43(\mathrm{~s}, 4 \mathrm{H}), 3.33(\mathrm{~s}, 6 \mathrm{H}), 2.50(\mathrm{~d}, J=1.6 \mathrm{~Hz}$, 2H), $2.13(\mathrm{~s}, 2 \mathrm{H}), 1.69(\mathrm{~s}, 3 \mathrm{H}), 1.65(\mathrm{~s}, 6 \mathrm{H})$.

${ }^{13} \mathbf{C ~ N M R}\left(\mathbf{C D C l}_{3}\right) \delta=201.2,134.5(\mathrm{~d}, J=13.8 \mathrm{~Hz}), 131.5,130.5,130.2,129.2(\mathrm{~d}, J=11.2$ $\mathrm{Hz}), 93.2,92.4,75.1,59.4,42.6,36.2,22.2,21.1$.

${ }^{31} \mathbf{P}$ NMR $\left(\mathrm{CDCl}_{3}\right) \delta=43.0$.

IR (neat) 2362, 1479, 1435, 1194, 1099, 1026, 997, $968 \mathrm{~cm}^{-1}$.

HRMS calculated for $\left[\mathrm{C}_{33} \mathrm{H}_{38} \mathrm{O}_{2} \mathrm{AuNaP}\right]^{+}: 717.2167$, found: 717.2174 .

\section{Gold Complex 20}

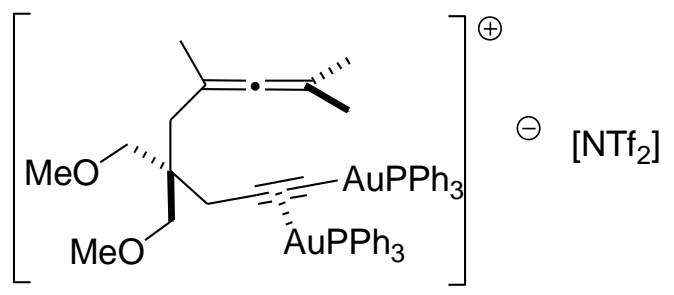

20

An oven-dried, sealed NMR tube was introduced in a glove-box and loaded with the gold acetylide precursor $\left(8.7 \mathrm{mg}, 12.5 \mu \mathrm{mol}, 1\right.$ equiv.) and $\left[\mathrm{Ph}_{3} \mathrm{PAu}\right] \mathrm{NTf}_{2} \bullet 1 / 2$ toluene $(9.8 \mathrm{mg}, 6.3$ $\mu$ mol, 0.5 equiv.). Outside the glove-box $\mathrm{CDCl}_{3}(5 \mathrm{~mL})$ dried over $\mathrm{K}_{2} \mathrm{CO}_{3}$ for several days was introduced through the septum cap of the tube. The solution turned rapidly into a light brown mixture. Formation of the digold complex 20 was checked by ${ }^{1} \mathrm{H}$ and ${ }^{31} \mathrm{P}$ NMR and occurs instantaneously.

${ }^{1}$ H NMR (400 MHz, CDCl $) \delta 7.59-7.49$ (m, 9H), $7.44-7.34$ (m, 21H), 7.28 - 7.23 (m, toluene), $7.21-7.13(\mathrm{~m}, 0 \mathrm{H}), 3.24(\mathrm{~s}, 4 \mathrm{H}), 3.18(\mathrm{~s}, 6 \mathrm{H}), 2.89(\mathrm{~s}, 2 \mathrm{H}), 2.38(\mathrm{~s}, 2 \mathrm{H}), 2.35$ (s, toluene), $2.01(\mathrm{~s}, 3 \mathrm{H}), 1.53(\mathrm{~s}, 6 \mathrm{H})$.

${ }^{13}$ C NMR (100 MHz, CDCl $) \delta 201.9\left(\mathrm{t}, J_{\mathrm{P}-\mathrm{C}}=2.7 \mathrm{~Hz}, \mathrm{C}_{\text {allene }}\right), 156.9$ (t, $J_{\mathrm{P}-\mathrm{C}}=55.1 \mathrm{~Hz}$, $\left.\mathrm{C}(\mathrm{Au})_{2}\right), 141.9$ (t, $\left.J_{\mathrm{P}-\mathrm{C}}=2.5 \mathrm{~Hz}, \mathrm{C}(\mathrm{Me})_{2}\right), 138.0$ (toluene), $134.0\left(\mathrm{~d}, J_{\mathrm{P}-\mathrm{C}}=14.0 \mathrm{~Hz}, 12 \mathrm{C}\right.$ $\left.\mathrm{PPh}_{3}\right), 132.4\left(6 \mathrm{C}-\mathrm{PPh}_{3}\right), 131.4\left(\mathrm{t}, J_{\mathrm{P}-\mathrm{C}}=4.6 \mathrm{~Hz}, \mathrm{CMe}\right), 130.2$ (t, $J=6.0 \mathrm{~Hz}$, not attributed), $129.7\left(\mathrm{~d}, J_{\mathrm{P}-\mathrm{C}}=11.8 \mathrm{~Hz}, 12 \mathrm{C}-\mathrm{PPh}_{3}\right), 129.1$ (toluene), $128.8\left(\mathrm{~d}, J_{\mathrm{P}-\mathrm{C}}=64.8 \mathrm{~Hz}, 6 \mathrm{C}-\mathrm{PPh}_{3}\right)$, 128.4 (toluene), 125.4 (toluene), 120.1 (q, $\left.J_{\mathrm{C}-\mathrm{F}}=322.0 \mathrm{~Hz}, 2 \mathrm{CF}_{3}\right), 75.9\left(2 \mathrm{C}, \mathrm{CH}_{2} \mathrm{O}\right), 61.7$ (t, $\left.J_{\mathrm{P}-\mathrm{C}}=2.0 \mathrm{~Hz}, \mathrm{C}_{\text {alkyne }}\right), 59.5\left(2 \mathrm{C}, \mathrm{OCH}_{3}\right), 43.1(\mathrm{C}), 34.9\left(\mathrm{CH}_{2}\right), 31.1\left(\mathrm{CH}_{2}\right), 28.3\left(2 \mathrm{CH}_{3}\right), 21.6$ (toluene), $18.0\left(\mathrm{CH}_{3}\right)$.

${ }^{31}$ P NMR (162 MHz, $\left.\mathbf{C D C l}_{3}\right) \delta 37.5$. 


\section{DFT calculations, including Figures S1-S5, Tables S1-S9 and Schemes S1-S2}

The Gaussian 03 ( $\mathrm{v}$ C02) set of programs was used for the geometry optimization of all structures. ${ }^{6}$ The optimizations were performed with the B3LYP functional, the LANL2DZ(ECP) basis set for Au, and the 6-31G(d,p) for other atoms. Stationary points were characterized as minima or transition states by calculating the Hessian matrix analytically at this level of theory. Thermodynamic corrections were taken from these calculations. The standard state for all thermodynamic data is $298.15 \mathrm{~K}$ and $1 \mathrm{~atm}$. Energies were refined by single point calculation at B2PLYP/def2-TZVP level using the ORCA program (version 3.0.3). Solvent correction for dichloromethane was obtained at the same level of theory using the COSMO model. All relative energies presented in the manuscript are Gibbs free energies $\left(\Delta G_{298}\right)$ or Gibbs free energies in dichloromethane $(\Delta G \mathrm{~s})$ in kilocalories per mole $(\mathrm{kcal} / \mathrm{mol})$.

Justification of the computational method used:

Recent literature reports (references cited in ref 27 of the manuscript) recommend the B2PLYP/def2-TZVP//BP86/def2-TZVP level for gold complexes. We show in this section that in our case, computational time can be saved by using a more modest level for geometry optimizations, i.e. the B3LYP/LANL2DZ(Au),6-31G(d,p). This is especially important here since some of the complex studied exceed 90 atoms. We have compared, in a first set of calculations, the influence of functionals and basis sets with the $\mathrm{PMe}_{3}$ ligand. A full geometry optimization of all intermediates and TS described in Scheme 7 was carried out at the BP86/def2-TZVP level and the results were compared with geometries (in parenthesis) at the B3LYP/LANL2DZ(Au),6-31G(d,p) level (Scheme S1). 

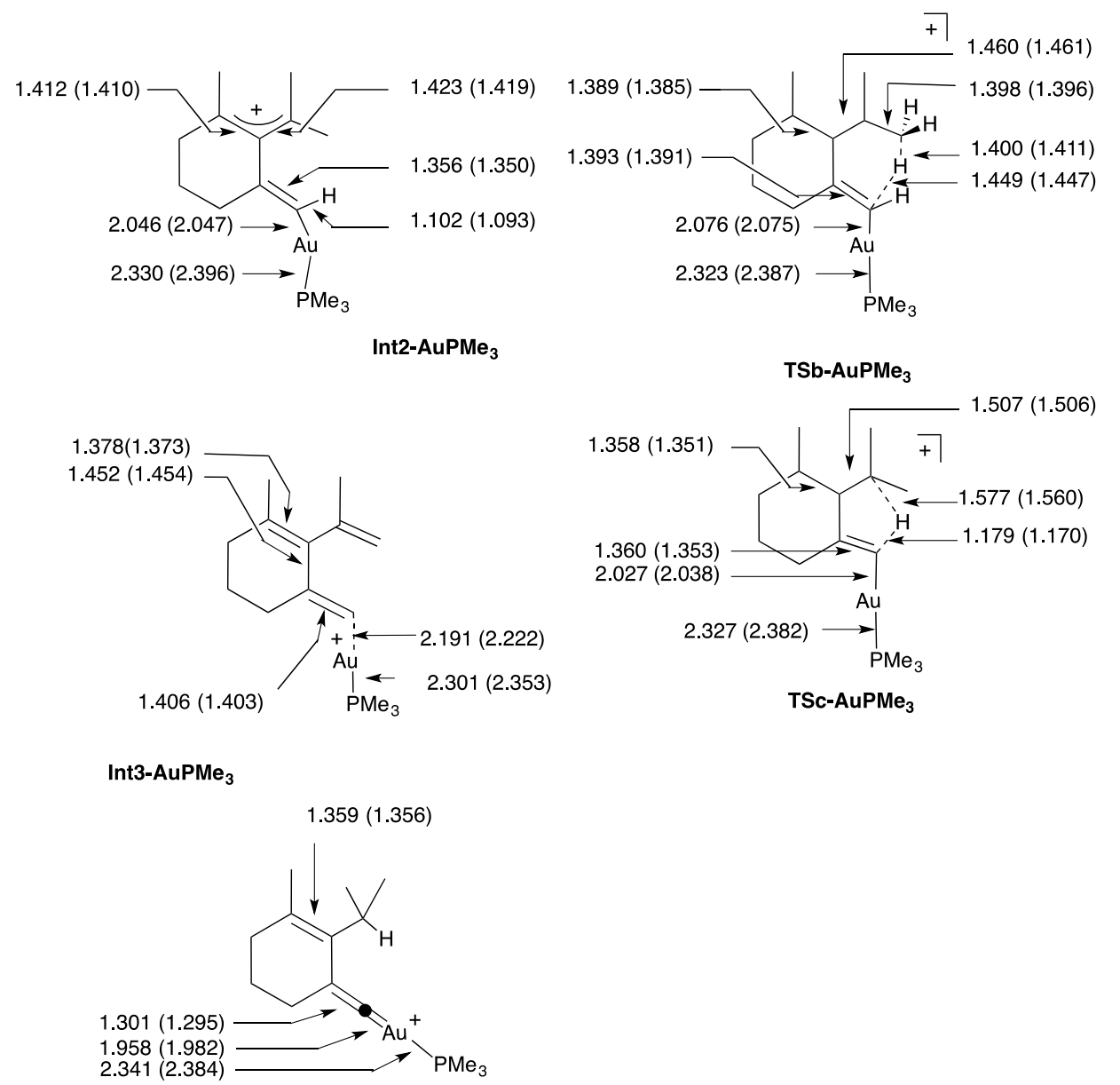

Int4-AuPMe ${ }_{3}$

Selected bond distances (A) for some crucial intermediates and TS: values at BP86/def2-TZVP level, compare to values at $\left(B 3 L Y P / L A N 2 D Z(6-31 G(d, p))\right.$ level, full optimisaton for $\mathrm{PMe}_{3}$ ligand

Scheme S1. Selected bond distances $(\AA)$ for some crucial intermediates and TS: values at the BP86/def2-TZVP level, compared to values (in parenthesis) at the B3LYP/LANL2DZ(ECP), 6-31G(d,p) level, full optimization for $\mathrm{PMe}_{3}$ ligand.

As we can observe, differences are not significant, the largest variation concerning the $\mathrm{Au}-\mathrm{P}$ bond (less than $0.06 \AA$ ).

Starting from these geometries, relative energies were obtained after a single point at B2PLYP/def2-TZVP level. Relative energies resulting from these calculations were compared with relatives energies computed at B2PLYP/def2-TZVP//B3LYP/LANL2DZ(Au),6$31 \mathrm{G}(\mathrm{d}, \mathrm{p})$ level (see Table S1 below)

Table S1. Comparison of Relative Energies for $\mathrm{Me}_{3} \mathrm{P}-\mathrm{Au}-$ allenyne 1 system.

\begin{tabular}{|c|c|c|c|c|c|c|c|c|c|c|c|}
\hline $\mathrm{Me}_{3} \mathrm{PAu}^{+}$ & Int1 & TSa & Int2 & TSb & Int3 & TSc & Int4 & TSd & Int5 & TSe & Int6 \\
\hline $\begin{array}{l}\text { B2PLYP/def2- } \\
\text { TZVP//B3LYP/LANL } \\
\text { 2DZ(Au),6-31G(d,p) }\end{array}$ & & 9.2 & -16.7 & 8.8 & -49.0 & -8.5 & -11.9 & -9.7 & -17.0 & -12.7 & -65.2 \\
\hline $\begin{array}{l}\text { B2PLYP/def2- } \\
\text { TZVP//BP 86/def2- } \\
\text { TZVP }\end{array}$ & & 9.9 & -15.9 & 9.6 & -48.5 & -7.5 & -12.1 & -8.3 & -14.2 & -11.7 & -65.0 \\
\hline
\end{tabular}


The energetic values differ weakly (average of less than $1 \mathrm{kcal} / \mathrm{mol}$, except for Int5). Thus, to gain computational time, we have preferred the B3LYP approach. Thus, Gibbs free energies reported in this work come from a single point calculation at B2PLYP/def2TZVP//B3LYP/LANL2DZ(Au),6-31G(d,p) level.

Table S2. (Table 5). Computed Gibbs Free Energies and solvent-corrected Gibbs Free Energies $298 \mathrm{~K} \quad$ (B2PLYP/def2-TZVP//B3LYP/LANL2DZ(Au), 6-31G(d,p), kcal.mol ${ }^{-1}$ ), Relative to Int1 (or Relative to Intx in parentheses)

\begin{tabular}{ccccccccccc}
\hline $\mathrm{M}$ & TSa & Int2 & TSb(rel. to Int2) & Int3 & TSc(rel. to Int2) & Int4 & TSd(rel. to Int4) & Int5 & TSe(rel. to Int5) & Int6 \\
\hline $\mathrm{H}_{3} \mathrm{PAu}^{+}$ & 4.4 & -22.1 & $1.4(23.5)$ & -49.7 & $-11.5(10.6)$ & -15.3 & $-9.3(6.0)$ & -17.9 & $-14.9(3.0)$ & -62.6 \\
& 0.6 & -32.4 & $-1.3(31.1)$ & -60.6 & $-21.2(11.2)$ & -27.4 & $-22.6(4.8)$ & -28.7 & $-25.9(2.8)$ & -79.7 \\
$\mathrm{Me}_{3} \mathrm{PAu}+$ & 12.2 & -10.7 & $12.1(22.8)$ & -42.6 & $-3.6(7.1)$ & -7.3 & $-2.9(4.4)$ & -11.3 & $-6.5(4.8)$ & -56.1 \\
& 10.8 & -20.0 & $10.3(30.3)$ & -52.5 & $-9.1(10.9)$ & -16.3 & $-13.6(2.7)$ & -19.7 & $-15.0(4.7)$ & -71.8 \\
$\mathrm{Et}_{3} \mathrm{PAu}+$ & 13.7 & -7.5 & $14.9(22.4)$ & -41.3 & $-1.2(6.3)$ & -6.1 & $-1.1(5.0)$ & -12.4 & $-4.3(8.1)$ & -54.2 \\
& 10.8 & -20.8 & $9.7(30.5)$ & -53.0 & $-9.5(11.3)$ & -16.7 & $-14.0(2.7)$ & -22.8 & $-15.5(7.3)$ & -72.7 \\
$\mathrm{Ph}_{3} \mathrm{PAu}+$ & 15.9 & -7.6 & $16.5(24.1)$ & -41.2 & $-3.4(4.2)$ & -6.2 & $-0.1(6.1)$ & -10.1 & $-2.5(7.6)$ & -56.1 \\
& 17.3 & -26.2 & $4.2(30.4)$ & -55.9 & $-13.9(12.3)$ & -15.6 & $-14.8(0.8)$ & -19.5 & $-13.8(5.7)$ & -74.4 \\
$\mathrm{tBu}_{3} \mathrm{PAu+}+$ & 14.7 & -6.4 & $16.2(22.6)$ & -40.9 & $-1.2(5.2)$ & -6.2 & $-1.3(4.9)$ & -10.0 & $-1.7(8.3)$ & -53.3 \\
& 11.1 & -19.3 & $10.6(29.9)$ & -52.7 & $-8.0(11.3)$ & -16.4 & $-13.6(2.8)$ & -19.8 & $-16.4(3.4)$ & -72.7 \\
$\mathrm{IPrAu}_{+}$ & 13.9 & -6.8 & $14.8(21.6)$ & -43.4 & $-1.5(5.3)$ & -8.7 & $-3.8(4.9)$ & -13.3 & $-5.0(8.3)$ & -59.0 \\
& 10.0 & -23.4 & $8.4(31.8)$ & -54.5 & $-10.8(12.6)$ & -18.0 & $-15.4(2.6)$ & -21.0 & $-13.4(7.6)$ & -76.7 \\
$\mathrm{C}_{4} \mathrm{Au}+$ & 12.4 & -8.2 & $13.3(21.5)$ & -41.6 & $-1.3(6.9)$ & -8.2 & $-2.1(6.1)$ & -10.8 & $-3.9(6.9)$ & -52.0 \\
& 12.4 & -21.5 & $8.7(30.2)$ & -53.3 & $-8.8(12.7)$ & -18.7 & $-14.8(3.9)$ & -21.3 & $-14.8(6.5)$ & -70.3 \\
$\mathrm{AuCl}_{3}$ & 2.6 & -22.6 & $-2.5(20.1)$ & -50.4 & $-15.8(6.8)$ & -16.1 & $-6.2(9.9)$ & -19.4 & $-15.3(4.1)$ & -66.7 \\
& -8.5 & -58.5 & $-28.3(30.2)$ & -68.5 & $-33.3(25.2)$ & -24.5 & $-14.9(9.6)$ & -27.3 & $-33.9(-6.6)$ & -91.2 \\
\hline
\end{tabular}




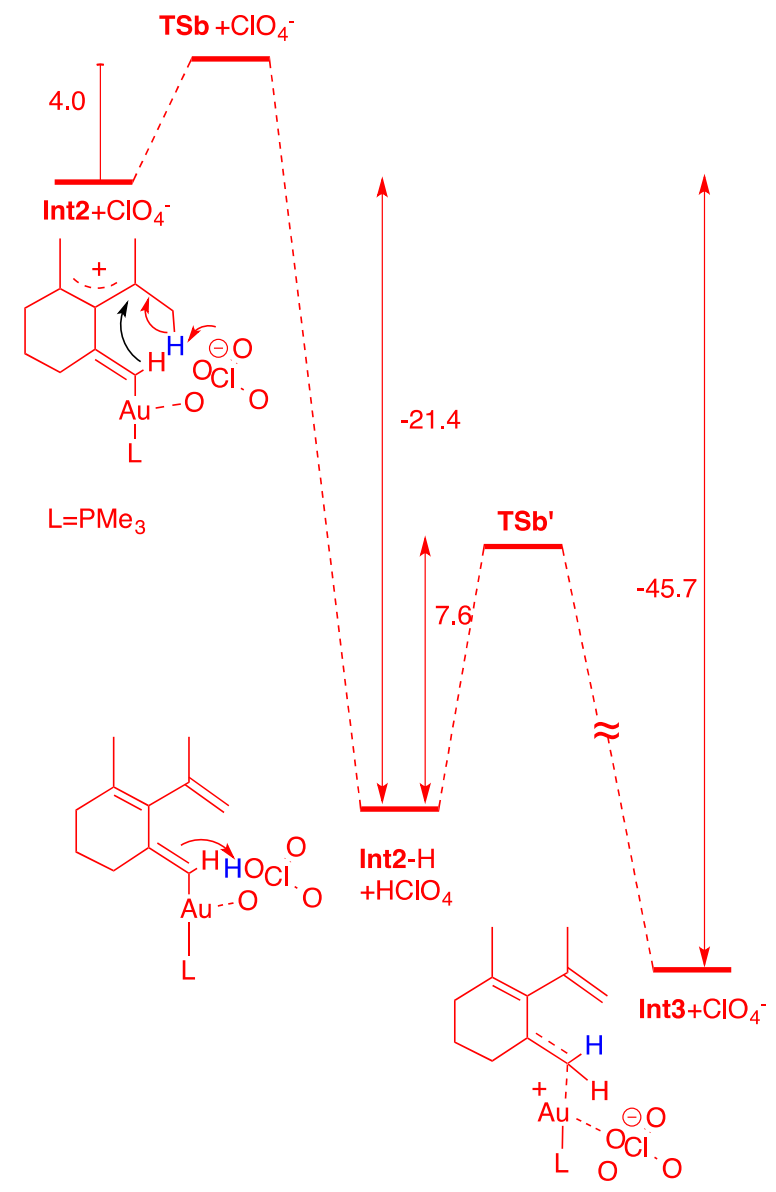

Scheme S2. Mechanistic Pathways Including $\mathrm{ClO}_{4}{ }^{-}$Counterion for ${ }^{+} \mathrm{Au}-\mathrm{PMe}_{3}$ catalyst

Table S3 (Scheme 13). Computed Gibbs Free Energies at 298 K (B2PLYP/def2

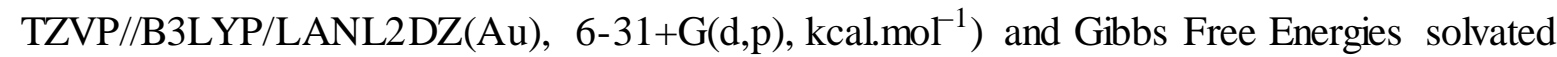
for the [2+2] Gold-Acetylide - Allene Cycloaddition of a 1,6 Allenyne (Relative to Int7)

\begin{tabular}{ccccccc}
\hline Int7 & TSf & Int8 & TSg & Int9 & TSh & Int10 \\
0 & 17.6 & -15.4 & 14.5 & -41.4 & -3.6 & -32.2 \\
& 18.9 & -16.4 & 15.6 & -40.2 & -5.4 & -34.2 \\
\hline
\end{tabular}

Table S4 (scheme 14). Computed Gibbs Free Energies at 298 K (B2PLYP/def2TZVP//B3LYP/LANL2DZ(Au), 6-31+G(d,p), kcal.mol ${ }^{-1}$ ) and Gibbs Free Energies solvated for the [2+2] Gold Acetylide - Allene Cycloaddition of a 1,5-Allenyne (Relative to Int11)

\begin{tabular}{ccccccccc}
\hline Int11 & TSi & Int12 & TSj & Int13 & TSk & Int14 & TSI & Int15 \\
0 & 13.4 & 3.3 & 14.5 & -38.0 & 1.7 & -5.1 & 9.5 & -17.2 \\
& 16.0 & 3.1 & 16.0 & -37.5 & 2.7 & -5.8 & 11.8 & -15.4 \\
\hline
\end{tabular}



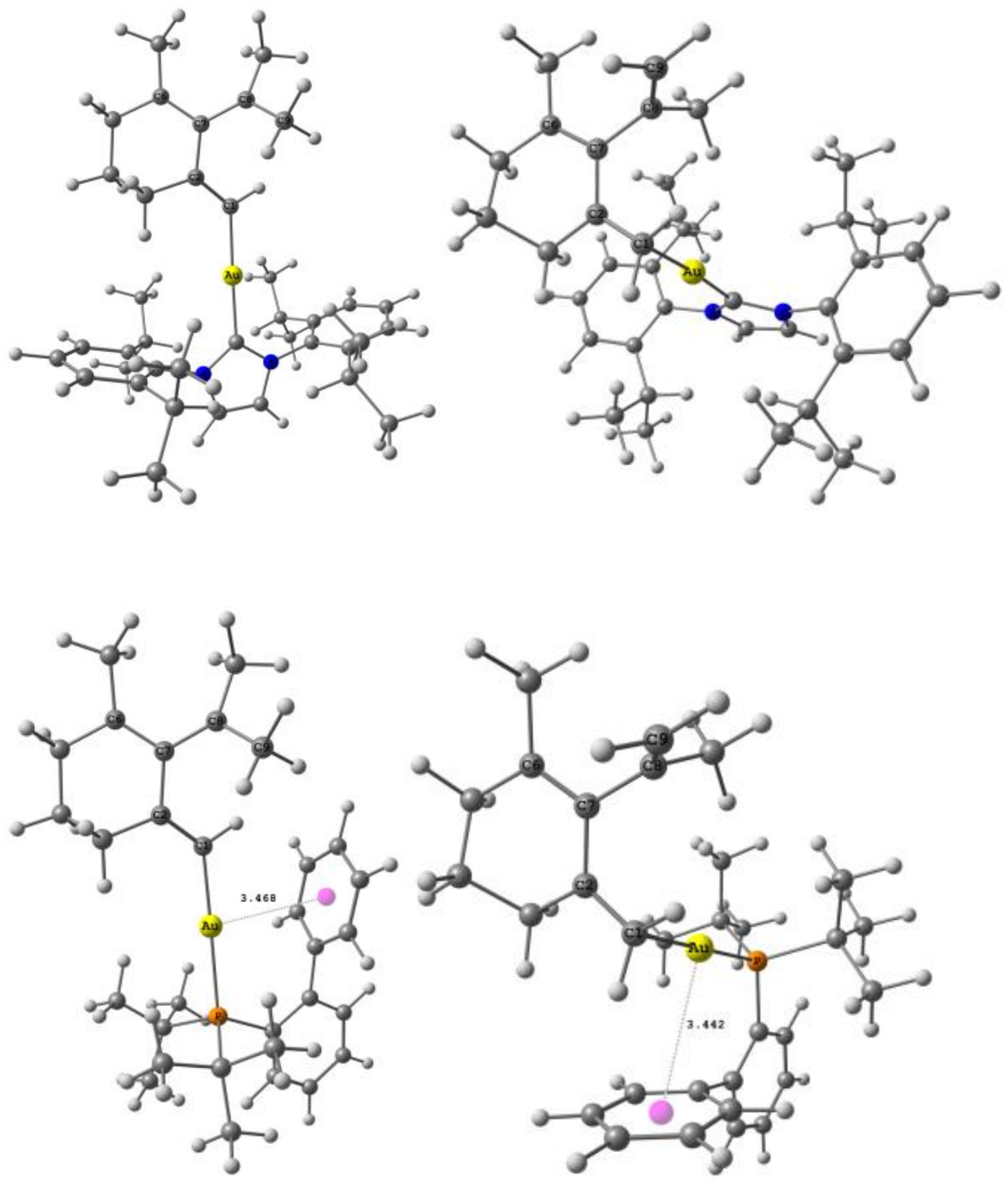

Figure S1. Geometries of the Computed Int2 (left) and Int3 (right) Complexes with $\mathrm{M}=$ $\mathrm{IPrAu}^{+}$and $\mathrm{C4Au}^{+}$(Distances in $\AA$ ). 

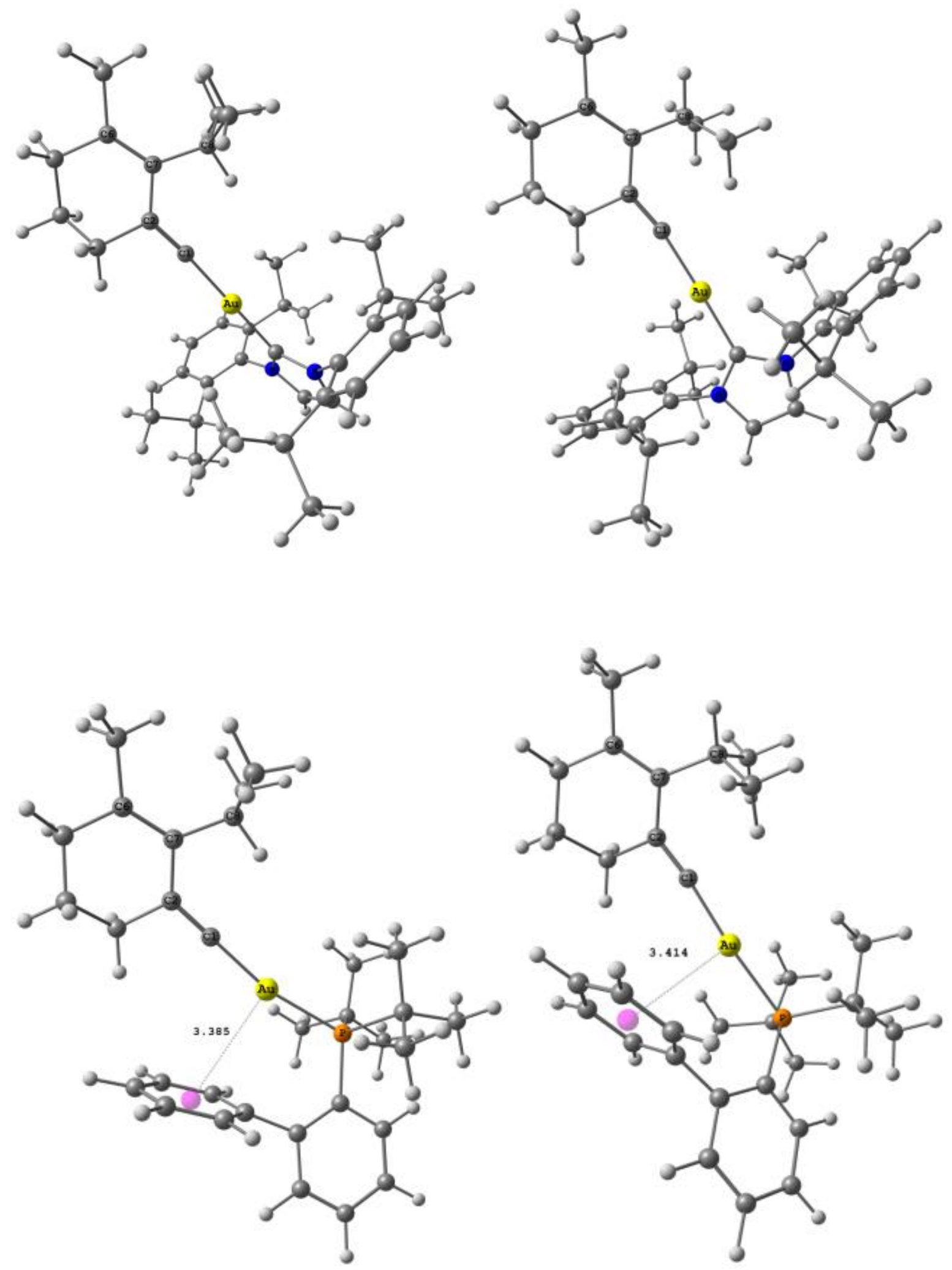

Figure S2. Geometries of the Computed Int4 (left) and Int5 (right) with $\mathrm{M}=\mathrm{IPrAu}^{+}$and $\mathrm{C4} \mathrm{Au}^{+}$(Distances in $\AA$ ). 

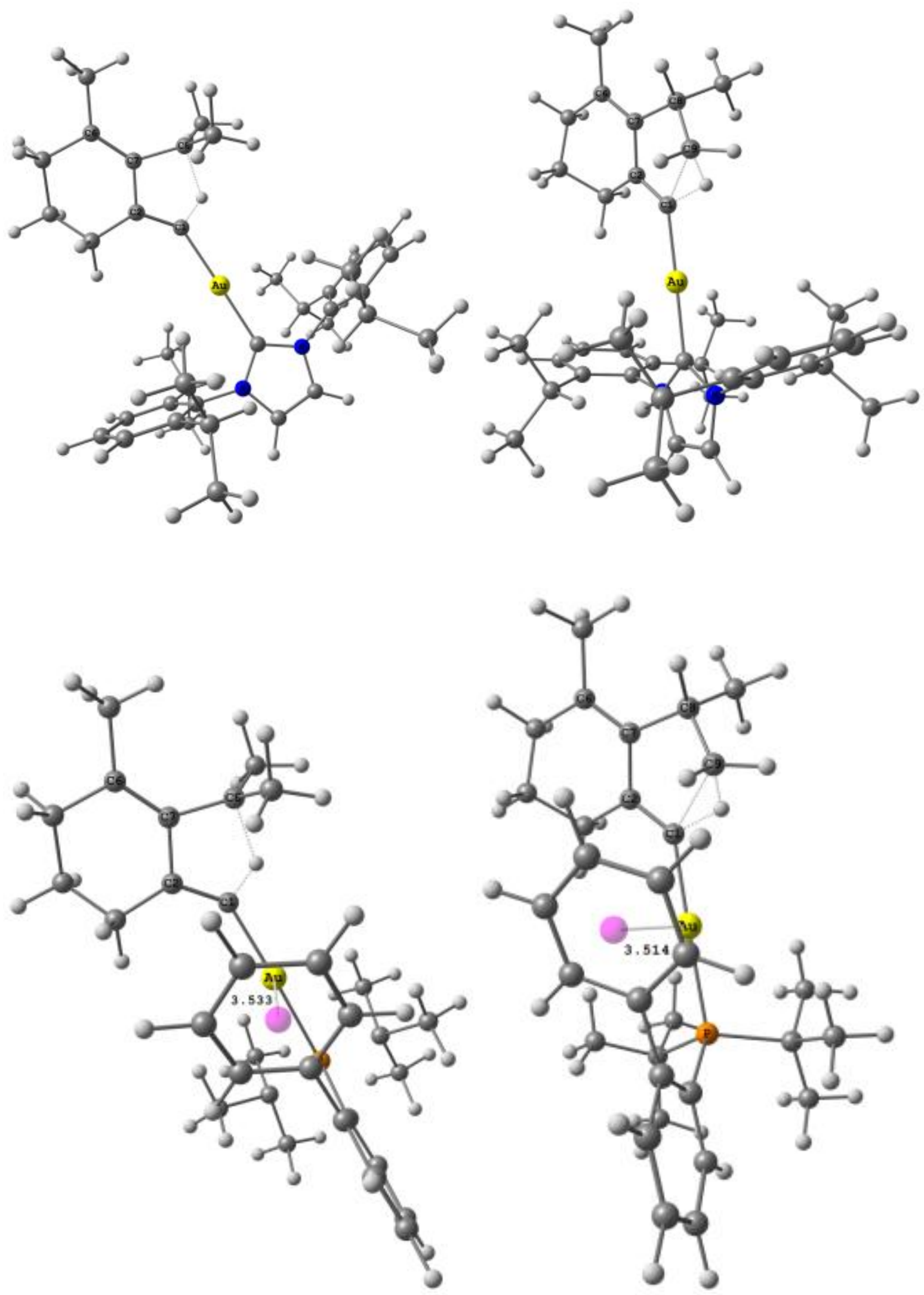

Figure S3. Geometries of the Computed TSc (left) and TSe (right) with $\mathrm{M}=\mathrm{IPrAu}^{+}$and $\mathrm{C}_{4} \mathrm{Au}^{+}$(Distances in $\AA$ ). 

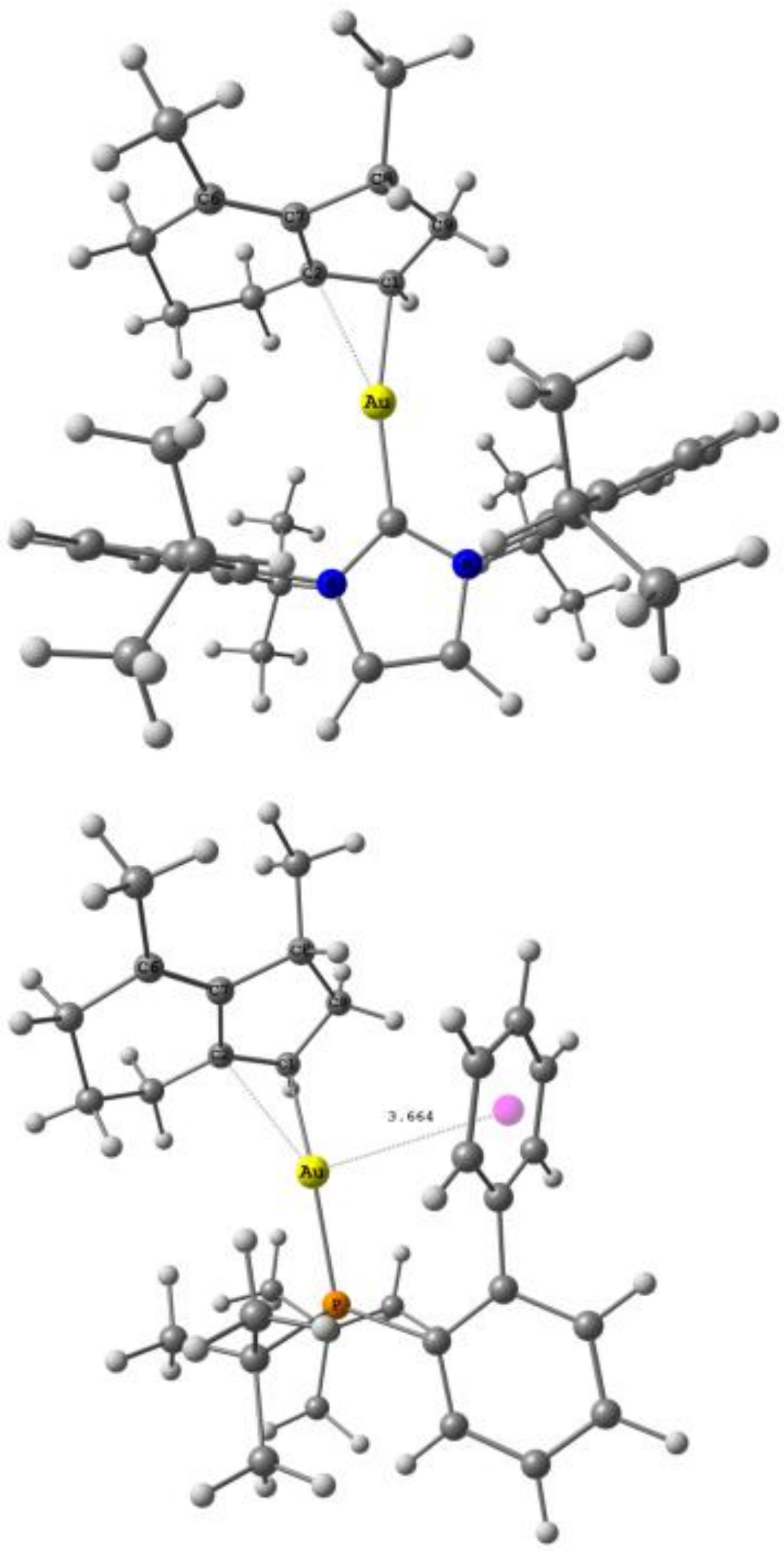

Figure S4. Geometries of the Computed Int6 with $\mathrm{M}=\mathrm{IPrAu}^{+}$(above) and $\mathbf{C 4 A u ^ { + }}$ (below) (Distances in $\AA$ ). 

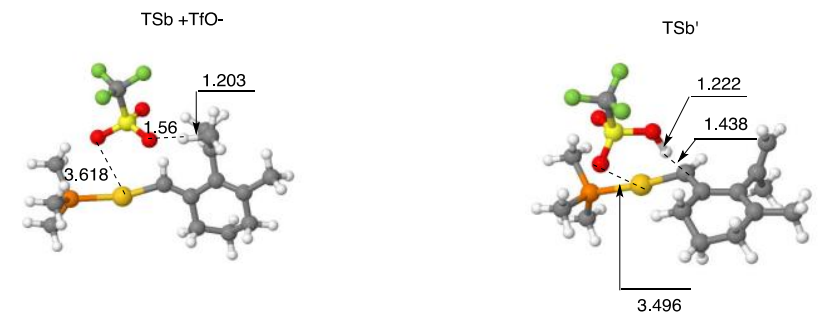

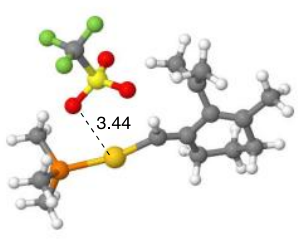

Int2 +TfO-

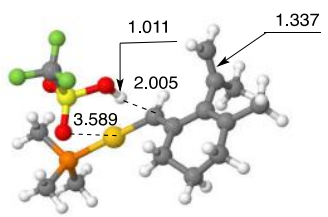

Int2- $\mathrm{H}, \mathrm{TfOH}$

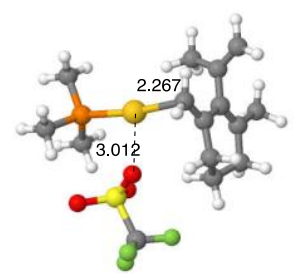

Int3 + TfO-

Figure S5. Geometries of the Computed triflate shuttle (scheme 9) with $\mathrm{M}=\mathrm{Me}_{3} \mathrm{PAu}^{+}$

By considering intermediate 2 (see Figure S6), we can anticipate that an adequate positioning of the cationic $\pi$ orbital centered on the $\mathrm{Ch}$ atom and the $\mathrm{Hh}$ to be transfered on $\mathrm{Ch}$, can highly favor the formation of the hydrindiene compound. When the $\pi$ cationic orbital is strongly conjugated with the double bond $\mathrm{C} 6-\mathrm{C} 7$, as for trisubstituted allenyne 12, the formation of the Alder-ene compound is favored. In the case where $\mathrm{H}$ is replaced by a $\mathrm{Me}$ group (allenyne 5), the conjugation is partially lost due to an effective $\mathrm{A}(1,3)$ strain, favoring the hydrindiene formation.

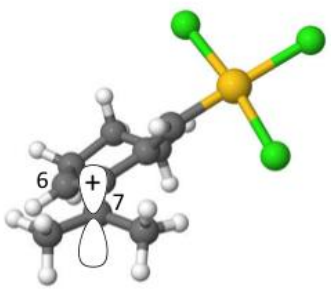

Int2H-AuCl3

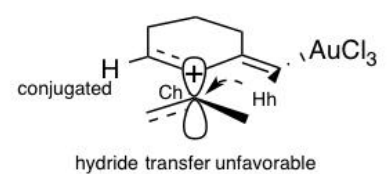

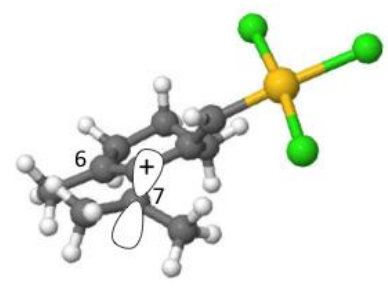

Int2-AuCl3

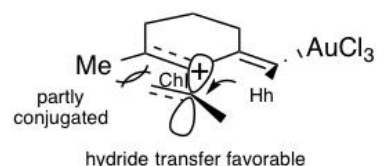

Figure S6. Geometries of the Computed Int2 and Int2H with $\mathrm{M}=\mathrm{AuCl}_{3}$ 
Table S5. Selected Bond Lengths ( $)$ of the Computed Complexes Int2 and Int3

\begin{tabular}{|c|c|c|c|c|c|c|c|c|}
\hline Int & M & $\mathrm{Au}-\mathrm{X}^{\lfloor\mathrm{a}\rfloor}$ & $\mathrm{Au}-\mathrm{C} 1$ & $\mathrm{C} 1-\mathrm{C} 2$ & $\mathrm{C} 2-\mathrm{C} 7$ & C7-C6 & C7-C8 & C8-C9 \\
\hline \multirow{8}{*}{ Int2 } & $\mathrm{H}_{3} \mathrm{PAu}^{+}$ & 2.402 & 2.035 & 1.349 & 1.500 & 1.411 & 1.419 & 1.493 \\
\hline & $\mathrm{Me}_{3} \mathrm{PAu}^{+}$ & 2.396 & 2.048 & 1.351 & 1.498 & 1.410 & 1.419 & 1.493 \\
\hline & $\mathrm{Et}_{3} \mathrm{PAu}^{+}$ & 2.399 & 2.051 & 1.351 & 1.498 & 1.410 & 1.419 & 1.493 \\
\hline & $\mathrm{Ph}_{3} \mathrm{PAu}^{+}$ & 2.405 & 2.048 & 1.351 & 1.497 & 1.410 & 1.419 & 1.493 \\
\hline & $t \mathrm{Bu}_{3} \mathrm{PAu}^{+}$ & 2.425 & 2.054 & 1.352 & 1.497 & 1.410 & 1.420 & 1.493 \\
\hline & $\mathrm{IPrAu}^{+}$ & 2.075 & 2.046 & 1.354 & 1.494 & 1.410 & 1.421 & 1.493 \\
\hline & $\mathrm{C}_{4} \mathrm{Au}^{+}$ & 2.424 & 2.047 & 1.352 & 1.495 & 1.410 & 1.421 & 1.493 \\
\hline & $\mathrm{AuCl}_{3}$ & 2.417 & 2.026 & 1.342 & 1.489 & 1.398 & 1.424 & 1.489 \\
\hline \multirow{8}{*}{ Int3 } & $\mathrm{H}_{3} \mathrm{PAu}^{+}$ & 2.361 & 2.179 & 1.415 & 1.447 & 1.378 & 1.507 & 1.338 \\
\hline & $\mathrm{Me}_{3} \mathrm{PAu}^{+}$ & 2.353 & 2.222 & 1.403 & 1.455 & 1.373 & 1.507 & 1.338 \\
\hline & $\mathrm{Et}_{3} \mathrm{PAu}^{+}$ & 2.354 & 2.235 & 1.400 & 1.457 & 1.372 & 1.507 & 1.338 \\
\hline & $\mathrm{Ph}_{3} \mathrm{PAu}^{+}$ & 2.358 & 2.237 & 1.399 & 1.459 & 1.371 & 1.507 & 1.338 \\
\hline & $t \mathrm{Bu}_{3} \mathrm{PAu}^{+}$ & 2.381 & 2.254 & 1.397 & 1.459 & 1.371 & 1.506 & 1.338 \\
\hline & $\mathrm{IPrAu}^{+}$ & 2.038 & 2.239 & 1.396 & 1.463 & 1.369 & 1.507 & 1.338 \\
\hline & $\mathrm{C} 4 \mathrm{Au}^{+}$ & 2.376 & 2.244 & 1.396 & 1.461 & 1.370 & 1.506 & 1.338 \\
\hline & $\mathrm{AuCl}_{3}$ & 2.395 & 2.248 & 1.421 & 1.444 & 1.378 & 1.508 & 1.338 \\
\hline
\end{tabular}

[a] $\mathrm{X}$ is the atombond to $\mathrm{Au}$ in the $\mathrm{M}$ fragment $(\mathrm{P}, \mathrm{C}$, or trans- $\mathrm{Cl})$.

Table S6. Selected Bond Lengths $(\AA)$ and Angles $\left(^{\circ}\right)$ of the Computed Complexes Int4 and Int5

\begin{tabular}{|c|c|c|c|c|c|c|c|c|}
\hline Int & M & $\mathrm{Au}-\mathrm{X}^{\lfloor\mathrm{a}\rfloor}$ & $\mathrm{Au}-\mathrm{C} 1$ & $\mathrm{C} 1-\mathrm{C} 2$ & $\mathrm{C} 2-\mathrm{C} 7$ & C7-C6 & $\mathrm{XAuCl}^{\lfloor\mathrm{a}\rfloor}$ & $\mathrm{AuC} 1 \mathrm{C} 2$ \\
\hline \multirow{8}{*}{ Int 4} & $\mathrm{H}_{3} \mathrm{PAu}^{+}$ & 2.386 & 1.971 & 1.294 & 1.465 & 1.357 & 179.4 & 174.8 \\
\hline & $\mathrm{Me}_{3} \mathrm{PAu}^{+}$ & 2.383 & 1.982 & 1.296 & 1.467 & 1.356 & 179.3 & 174.9 \\
\hline & $\mathrm{Et}_{3} \mathrm{PAu}^{+}$ & 2.385 & 1.984 & 1.296 & 1.468 & 1.356 & 179.6 & 174.8 \\
\hline & $\mathrm{Ph}_{3} \mathrm{PAu}^{+}$ & 2.390 & 1.979 & 1.297 & 1.468 & 1.356 & 179.3 & 173.9 \\
\hline & $t \mathrm{Bu}_{3} \mathrm{PAu}^{+}$ & 2.411 & 1.982 & 1.298 & 1.469 & 1.356 & 179.0 & 174.3 \\
\hline & $\mathrm{IPrAu}^{+}$ & 2.054 & 1.961 & 1.299 & 1.472 & 1.355 & 179.6 & 175.0 \\
\hline & $\mathrm{C} 4 \mathrm{Au}^{+}$ & 2.414 & 1.969 & 1.300 & 1.472 & 1.356 & 167.6 & 178.2 \\
\hline & $\mathrm{AuCl}_{3}$ & 2.356 & 1.974 & 1.290 & 1.471 & 1.356 & 177.7 & 174.6 \\
\hline \multirow{8}{*}{ Int5 } & $\mathrm{H}_{3} \mathrm{PAu}^{+}$ & 2.379 & 1.980 & 1.293 & 1.470 & 1.357 & 179.6 & 170.8 \\
\hline & $\mathrm{Me}_{3} \mathrm{PAu}^{+}$ & 2.378 & 1.992 & 1.294 & 1.472 & 1.356 & 179.8 & 171.2 \\
\hline & $\mathrm{Et}_{3} \mathrm{PAu}^{+}$ & 2.381 & 1.994 & 1.294 & 1.473 & 1.356 & 179.4 & 171.8 \\
\hline & $\mathrm{Ph}_{3} \mathrm{PAu}^{+}$ & 2.386 & 1.989 & 1.294 & 1.473 & 1.356 & 179.3 & 171.6 \\
\hline & $t \mathrm{Bu}_{3} \mathrm{PAu}^{+}$ & 2.406 & 1.992 & 1.295 & 1.474 & 1.356 & 179.8 & 171.7 \\
\hline & $\operatorname{IPrAu}^{+}$ & 2.055 & 1.973 & 1.295 & 1.474 & 1.356 & 178.4 & 170.6 \\
\hline & $\mathrm{C} 4 \mathrm{Au}^{+}$ & 2.409 & 1.984 & 1.295 & 1.475 & 1.356 & 173.6 & 171.4 \\
\hline & $\mathrm{AuCl}_{3}$ & 2.356 & 1.979 & 1.287 & 1.474 & 1.356 & 179.4 & 170.4 \\
\hline
\end{tabular}

[a] $\mathrm{X}$ is the atombond to $\mathrm{Au}$ in the $\mathrm{M}$ fragment $(\mathrm{P}, \mathrm{C}$, or trans- $\mathrm{Cl})$. 
Table S7. Selected Bond Lengths ( $)$ of the Computed Species TSc and TSe

\begin{tabular}{|c|c|c|c|c|c|c|c|c|}
\hline TS & M & $\mathrm{Au}-\mathrm{X}^{\lfloor\mathrm{a}\rfloor}$ & $\mathrm{Au}-\mathrm{Cl}$ & $\mathrm{C} 1-\mathrm{C} 2$ & C2-C7 & C7-C6 & $\mathrm{C} 1-\mathrm{H}$ & C8-H \\
\hline \multirow{9}{*}{ TSc } & $\mathrm{H}_{3} \mathrm{PAu}^{+}$ & 2.384 & 2.021 & 1.349 & 1.448 & 1.349 & 1.198 & 1.462 \\
\hline & $\mathrm{Me}_{3} \mathrm{PAu}^{+}$ & 2.382 & 2.038 & 1.353 & 1.447 & 1.351 & 1.170 & 1.560 \\
\hline & $\mathrm{Et}_{3} \mathrm{PAu}^{+}$ & 2.385 & 2.042 & 1.354 & 1.448 & 1.352 & 1.165 & 1.586 \\
\hline & $\mathrm{Ph}_{3} \mathrm{PAu}^{+}$ & 2.391 & 2.039 & 1.354 & 1.447 & 1.352 & 1.164 & 1.586 \\
\hline & $t \mathrm{Bu}_{3} \mathrm{PAu}^{+}$ & 2.413 & 2.045 & 1.356 & 1.448 & 1.352 & 1.159 & 1.616 \\
\hline & $\mathrm{IPrAu}^{+}$ & 2.064 & 2.034 & 1.359 & 1.446 & 1.353 & 1.152 & 1.651 \\
\hline & $\mathrm{C} \mathrm{Au}^{+}$ & 2.414 & 2.040 & 1.357 & 1.448 & 1.353 & 1.152 & 1.650 \\
\hline & $\mathrm{AuCl}_{3}$ & 2.392 & 2.036 & 1.352 & 1.452 & 1.350 & 1.164 & 1.521 \\
\hline & $\mathrm{M}$ & $\overline{A u}-X^{[a]}$ & $\mathrm{Au}-\mathrm{Cl}$ & C1-C2 & $\mathrm{C} 2-\mathrm{C} 7$ & C7-C6 & C1-H & C9-H \\
\hline \multirow{8}{*}{ TSe } & $\mathrm{H}_{3} \mathrm{PAu}^{+}$ & 2.374 & 2.037 & 1.355 & 1.461 & 1.357 & 1.197 & 1.329 \\
\hline & $\mathrm{Me}_{3} \mathrm{PAu}^{+}$ & 2.372 & 2.057 & 1.358 & 1.460 & 1.357 & 1.184 & 1.353 \\
\hline & $\mathrm{Et}_{3} \mathrm{PAu}^{+}$ & 2.374 & 2.062 & 1.359 & 1.461 & 1.356 & 1.180 & 1.360 \\
\hline & $\mathrm{Ph}_{3} \mathrm{PAu}^{+}$ & 2.379 & 2.058 & 1.359 & 1.461 & 1.356 & 1.181 & 1.360 \\
\hline & $t \mathrm{Bu}_{3} \mathrm{PAu}^{+}$ & 2.400 & 2.066 & 1.360 & 1.460 & 1.356 & 1.176 & 1.370 \\
\hline & $\mathrm{IPrAu}^{+}$ & 2.052 & 2.054 & 1.361 & 1.460 & 1.356 & 1.175 & 1.373 \\
\hline & $\mathrm{C} 4 \mathrm{Au}^{+}$ & 2.400 & 2.061 & 1.359 & 1.461 & 1.356 & 1.176 & 1.368 \\
\hline & $\mathrm{AuCl}_{3}$ & 2.374 & 2.025 & 1.331 & 1.468 & 1.353 & 1.259 & 1.247 \\
\hline
\end{tabular}

[a] $\mathrm{X}$ is the atombond to $\mathrm{Au}$ in the $\mathrm{M}$ fragment $(\mathrm{P}, \mathrm{C}$, or trans- $\mathrm{Cl})$.

Table S8. Selected Bond Lengths ( $)$ of the Computed Complexes Int6

\begin{tabular}{lllllll} 
Int & $\mathrm{M}$ & $\mathrm{Au}-\mathrm{X}^{[\mathrm{a}]}$ & $\mathrm{Au}-\mathrm{C} 1$ & $\mathrm{C} 1-\mathrm{C} 2$ & $\mathrm{C} 2-\mathrm{C} 7$ & $\mathrm{C} 7-\mathrm{C6}$ \\
\hline \multirow{6}{*}{ Int6 } & $\mathrm{H}_{3} \mathrm{PAu}^{+}$ & 2.367 & 2.207 & 1.411 & 1.444 & 1.367 \\
& $\mathrm{Me}_{3} \mathrm{PAu}^{+}$ & 2.356 & 2.258 & 1.398 & 1.453 & 1.361 \\
& $\mathrm{Et}_{3} \mathrm{PAu}^{+}$ & 2.357 & 2.270 & 1.395 & 1.455 & 1.360 \\
& $\mathrm{Ph}_{3} \mathrm{PAu}^{+}$ & 2.360 & 2.273 & 1.395 & 1.457 & 1.359 \\
& $t \mathrm{Bu}_{3} \mathrm{PAu}^{-}$ & 2.379 & 2.289 & 1.392 & 1.459 & 1.359 \\
& $\mathrm{IPrAu}^{+}$ & 2.036 & 2.275 & 1.390 & 1.463 & 1.356 \\
& $\mathrm{C4Au}^{+}$ & 2.386 & 2.295 & 1.393 & 1.455 & 1.359 \\
& $\mathrm{AuCl}_{3}$ & 2.398 & 2.271 & 1.418 & 1.430 & 1.371
\end{tabular}

[a] $\mathrm{X}$ is the atom bond to $\mathrm{Au}$ in the $\mathrm{M}$ fragment $(\mathrm{P}, \mathrm{C}$, or trans $-\mathrm{Cl})$.

Table S9. Coordinates $(\mathrm{x}, \mathrm{y}, \mathrm{z})$ of the computed species.

\begin{tabular}{|llll|llll|}
\hline & \multicolumn{3}{|c|}{ Int1 $\left(\mathbf{H}_{3} \mathbf{P A u}{ }^{+}\right)$} & \multicolumn{3}{c|}{ Int1 $\left(\mathbf{M e}_{3} \mathbf{P A u}{ }^{+}\right)$} \\
\hline $\mathrm{C}$ & -3.155068000 & 1.607138000 & 0.029670000 & $\mathrm{C}$ & -5.255239000 & -0.860674000 & 0.501273000 \\
$\mathrm{C}$ & -4.371752000 & -0.723244000 & -0.030914000 & $\mathrm{C}$ & -4.482366000 & 1.566615000 & -0.149024000 \\
$\mathrm{C}$ & -3.680606000 & 0.392967000 & -0.013843000 & $\mathrm{C}$ & -4.844412000 & 0.347121000 & 0.183609000 \\
$\mathrm{C}$ & -0.778443000 & -0.251136000 & 0.171484000 & $\mathrm{C}$ & -0.842953000 & -1.022295000 & -0.757716000 \\
$\mathrm{C}$ & -1.364583000 & -1.499450000 & 0.620967000 & $\mathrm{C}$ & -1.781421000 & 0.065189000 & -0.979309000 \\
$\mathrm{C}$ & -2.272379000 & -2.202746000 & -0.435087000 & $\mathrm{C}$ & -1.979911000 & 1.004553000 & 0.233768000 \\
$\mathrm{C}$ & -3.765860000 & -2.112343000 & -0.119149000 & $\mathrm{C}$ & -3.047354000 & 2.069150000 & -0.040305000 \\
\hline
\end{tabular}




\begin{tabular}{|c|c|c|c|c|c|c|c|}
\hline C & -5.887740000 & -0.689931000 & 0.056239000 & C & -5.489843000 & 2.598583000 & -0.618782000 \\
\hline c & -2.845973000 & 2.302117000 & 1.340167000 & C & -5.352131000 & -1.977583000 & -0.519593000 \\
\hline c & -2.924262000 & 2.415962000 & -1.226744000 & c & -5.689788000 & -1.210759000 & 1.910927000 \\
\hline $\mathrm{H}$ & -1.894850000 & -1.336519000 & 1.566353000 & $\mathrm{H}$ & -2.743269000 & -0.410664000 & -1.219913000 \\
\hline $\mathrm{H}$ & -0.506383000 & -2.143140000 & 0.865709000 & $\mathrm{H}$ & -1.480895000 & 0.629830000 & -1.870559000 \\
\hline $\mathrm{H}$ & -2.070015000 & -1.783827000 & -1.425435000 & H & -2.263833000 & 0.404738000 & 1.104087000 \\
\hline $\mathrm{H}$ & -1.988487000 & -3.257943000 & -0.474529000 & H & -1.024603000 & 1.490485000 & 0.465624000 \\
\hline $\mathrm{H}$ & -3.976456000 & -2.643801000 & 0.821052000 & $\mathrm{H}$ & -2.793362000 & 2.619805000 & -0.958267000 \\
\hline $\mathrm{H}$ & -4.310208000 & -2.670804000 & -0.891793000 & H & -3.005243000 & 2.816884000 & 0.763946000 \\
\hline $\mathrm{H}$ & -6.234535000 & -1.275011000 & 0.916696000 & $\mathrm{H}$ & -5.245572000 & 2.951821000 & -1.628624000 \\
\hline $\mathrm{H}$ & -6.265079000 & 0.328799000 & 0.157662000 & $\mathrm{H}$ & -6.501944000 & 2.190838000 & -0.632815000 \\
\hline $\mathrm{H}$ & -6.332610000 & -1.137916000 & -0.840297000 & $\mathrm{H}$ & -5.481785000 & 3.477320000 & 0.038463000 \\
\hline $\mathrm{H}$ & -1.808198000 & 2.656630000 & 1.368810000 & H & -4.732677000 & -2.834433000 & -0.224596000 \\
\hline $\mathrm{H}$ & -3.019119000 & 1.653145000 & 2.200797000 & H & -5.050114000 & -1.651391000 & -1.518150000 \\
\hline $\mathrm{H}$ & -3.483216000 & 3.188439000 & 1.446752000 & H & -6.383758000 & -2.344568000 & -0.583289000 \\
\hline $\mathrm{H}$ & -1.891319000 & 2.789584000 & -1.280521000 & $\mathrm{H}$ & -5.090047000 & -2.039531000 & 2.308330000 \\
\hline $\mathrm{H}$ & -3.563829000 & 3.307142000 & -1.219490000 & $\mathrm{H}$ & -6.734337000 & -1.545510000 & 1.915588000 \\
\hline $\mathrm{H}$ & -3.137185000 & 1.844024000 & -2.131360000 & $\mathrm{H}$ & -5.598343000 & -0.359631000 & 2.588496000 \\
\hline $\mathrm{H}$ & -0.172419000 & 1.675270000 & -0.613999000 & H & 0.248787000 & -2.976434000 & -0.493492000 \\
\hline $\mathrm{Au}$ & 1.998424000 & 0.082662000 & -0.039753000 & $\mathrm{Au}$ & 1.615879000 & -0.518230000 & -0.164340000 \\
\hline C & -0.030563000 & 0.677494000 & -0.222284000 & C & -0.045422000 & -1.946463000 & -0.569403000 \\
\hline$P$ & 4.300724000 & -0.382326000 & 0.086798000 & $\mathrm{P}$ & 3.611557000 & 0.604116000 & 0.321377000 \\
\hline $\mathrm{H}$ & 5.142123000 & 0.577609000 & -0.501655000 & C & 3.566802000 & 1.471165000 & 1.935674000 \\
\hline $\mathrm{H}$ & 4.848685000 & -0.498854000 & 1.376279000 & $\mathrm{H}$ & 4.527161000 & 1.958773000 & 2.128570000 \\
\hline \multirow[t]{11}{*}{$\mathrm{H}$} & 4.740150000 & -1.569167000 & -0.525512000 & $\mathrm{H}$ & 2.774968000 & 2.224115000 & 1.928842000 \\
\hline & & & & $\mathrm{H}$ & 3.359357000 & 0.753306000 & 2.732845000 \\
\hline & & & & C & 4.051193000 & 1.868492000 & -0.930631000 \\
\hline & & & & $\mathrm{H}$ & 3.263810000 & 2.624009000 & -0.983958000 \\
\hline & & & & $\mathrm{H}$ & 4.996580000 & 2.349932000 & -0.662605000 \\
\hline & & & & $\mathrm{H}$ & 4.150859000 & 1.396777000 & -1.911024000 \\
\hline & & & & C & 5.031320000 & -0.552758000 & 0.402637000 \\
\hline & & & & H & 5.949845000 & -0.006048000 & 0.636497000 \\
\hline & & & & H & 4.851317000 & -1.302560000 & 1.176737000 \\
\hline & & & & $\mathrm{H}$ & 5.148474000 & -1.061461000 & -0.557273000 \\
\hline & \multicolumn{3}{|c|}{ Int1 $\left(\mathrm{Et}_{3} \mathrm{PAu}^{+}\right)$} & \multicolumn{4}{|c|}{ Int1 $\left(\mathrm{Ph}_{3} \mathrm{PAu}^{+}\right)$} \\
\hline C & -5.777190000 & -0.631830000 & 0.525661000 & C & -5.818008000 & 1.680744000 & -0.163758000 \\
\hline C & -4.883835000 & 1.586482000 & -0.566086000 & C & -6.173241000 & -0.703976000 & 0.882532000 \\
\hline C & -5.306171000 & 0.467952000 & -0.019236000 & C & -5.975591000 & 0.484816000 & 0.358276000 \\
\hline C & -1.341811000 & -1.200560000 & -0.519236000 & c & -2.319830000 & -1.107874000 & -1.448155000 \\
\hline C & -2.221294000 & -0.142079000 & -0.989185000 & C & -3.259682000 & -1.308881000 & -0.350276000 \\
\hline C & -2.421005000 & 1.019767000 & 0.012055000 & C & -4.627854000 & -1.820386000 & -0.879198000 \\
\hline C & -3.432755000 & 2.048299000 & -0.503839000 & C & -5.643290000 & -1.988702000 & 0.256994000 \\
\hline C & -5.831612000 & 2.539724000 & -1.268603000 & C & -7.001938000 & -0.895262000 & 2.138399000 \\
\hline C & -5.890574000 & -1.932582000 & -0.245024000 & C & -4.673832000 & 2.592621000 & 0.234174000 \\
\hline C & -6.266774000 & -0.665505000 & 1.960041000 & C & -6.790494000 & 2.242646000 & -1.182013000 \\
\hline $\mathrm{H}$ & -3.192961000 & -0.619549000 & -1.179504000 & $\mathrm{H}$ & -3.408484000 & -0.365017000 & 0.184174000 \\
\hline
\end{tabular}




\begin{tabular}{|c|c|c|c|c|c|c|c|}
\hline H & -1.862227000 & 0.230100000 & -1.956660000 & $\mathrm{H}$ & -2.841872000 & -2.032467000 & 0.359861000 \\
\hline $\mathrm{H}$ & -2.760993000 & 0.609796000 & 0.968250000 & $\mathrm{H}$ & -5.012616000 & -1.111121000 & -1.618219000 \\
\hline $\mathrm{H}$ & -1.454595000 & 1.507150000 & 0.187236000 & $\mathrm{H}$ & -4.476548000 & -2.778454000 & -1.386127000 \\
\hline $\mathrm{H}$ & -3.128415000 & 2.397939000 & -1.501682000 & $\mathrm{H}$ & -5.213920000 & -2.620552000 & 1.048141000 \\
\hline $\mathrm{H}$ & -3.382730000 & 2.937894000 & 0.139524000 & $\mathrm{H}$ & -6.499361000 & -2.558167000 & -0.131288000 \\
\hline $\mathrm{H}$ & -5.544430000 & 2.673371000 & -2.319342000 & $\mathrm{H}$ & -6.405296000 & -1.358053000 & 2.934776000 \\
\hline $\mathrm{H}$ & -6.859947000 & 2.175839000 & -1.237119000 & H & -7.393258000 & 0.054056000 & 2.508258000 \\
\hline $\mathrm{H}$ & -5.802601000 & 3.531773000 & -0.800362000 & $\mathrm{H}$ & -7.849262000 & -1.565135000 & 1.944826000 \\
\hline $\mathrm{H}$ & -5.320523000 & -2.731252000 & 0.247021000 & H & -4.061692000 & 2.857331000 & -0.638033000 \\
\hline $\mathrm{H}$ & -5.540108000 & -1.833580000 & -1.275535000 & $\mathrm{H}$ & -4.030443000 & 2.134868000 & 0.990250000 \\
\hline $\mathrm{H}$ & -6.934898000 & -2.266514000 & -0.275008000 & $\mathrm{H}$ & -5.060140000 & 3.534277000 & 0.643495000 \\
\hline $\mathrm{H}$ & -5.715835000 & -1.415560000 & 2.541662000 & $\mathrm{H}$ & -6.270815000 & 2.505517000 & -2.112204000 \\
\hline $\mathrm{H}$ & -7.324820000 & -0.952744000 & 1.995119000 & H & -7.250635000 & 3.164231000 & -0.804545000 \\
\hline $\mathrm{H}$ & -6.157638000 & 0.303709000 & 2.450640000 & H & -7.585640000 & 1.532964000 & -1.418141000 \\
\hline $\mathrm{H}$ & -0.382550000 & -3.111640000 & 0.211020000 & H & -1.276401000 & -0.920652000 & -3.453219000 \\
\hline $\mathrm{Au}$ & 1.126671000 & -0.718236000 & -0.008185000 & $\mathrm{Au}$ & 0.059934000 & -0.463330000 & -0.964758000 \\
\hline c & -0.625776000 & -2.114424000 & -0.102005000 & C & -1.587708000 & -0.956307000 & -2.427859000 \\
\hline$P$ & 3.208801000 & 0.324602000 & 0.243143000 & $P$ & 2.040244000 & 0.086857000 & 0.165148000 \\
\hline C & 3.514494000 & 0.870817000 & 1.980184000 & C & 2.898397000 & 1.471004000 & -0.657805000 \\
\hline $\mathrm{H}$ & 4.522536000 & 1.299280000 & 2.018757000 & $\mathrm{H}$ & 2.564012000 & 0.658010000 & -2.637462000 \\
\hline $\mathrm{H}$ & 3.533552000 & -0.042166000 & 2.585483000 & C & 3.006205000 & 1.464768000 & -2.058577000 \\
\hline C & 3.354282000 & 1.803683000 & -0.858743000 & C & 3.684671000 & 2.491838000 & -2.711111000 \\
\hline $\mathrm{H}$ & 2.514937000 & 2.459940000 & -0.605191000 & C & 4.252636000 & 3.533910000 & -1.972862000 \\
\hline $\mathrm{H}$ & 3.162740000 & 1.444176000 & -1.875331000 & $\mathrm{H}$ & 3.765281000 & 2.482527000 & -3.793546000 \\
\hline C & 4.593817000 & -0.826519000 & -0.168715000 & $\mathrm{H}$ & 4.775410000 & 4.336884000 & -2.483182000 \\
\hline H & 5.532359000 & -0.292441000 & 0.019169000 & C & 4.144690000 & 3.545889000 & -0.581542000 \\
\hline $\mathrm{H}$ & 4.539898000 & -1.640640000 & 0.562343000 & $\mathrm{H}$ & 4.583184000 & 4.356047000 & -0.007394000 \\
\hline C & 4.688199000 & 2.559317000 & -0.781971000 & C & 3.469966000 & 2.518173000 & 0.080215000 \\
\hline $\mathrm{H}$ & 4.876679000 & 2.960543000 & 0.217643000 & $\mathrm{H}$ & 3.387157000 & 2.537060000 & 1.161854000 \\
\hline $\mathrm{H}$ & 5.535528000 & 1.928307000 & -1.064251000 & C & 0.599612000 & 1.455692000 & 2.125929000 \\
\hline H & 4.666334000 & 3.405329000 & -1.474646000 & H & -0.019894000 & 1.800272000 & 1.301922000 \\
\hline C & 4.550898000 & -1.378882000 & -1.599419000 & C & 0.323490000 & 1.879459000 & 3.424184000 \\
\hline $\mathrm{H}$ & 4.651122000 & -0.587546000 & -2.347818000 & H & -0.510502000 & 2.549859000 & 3.606867000 \\
\hline H & 5.376912000 & -2.079022000 & -1.749557000 & C & 1.115634000 & 1.437407000 & 4.487618000 \\
\hline $\mathrm{H}$ & 3.617519000 & -1.916311000 & -1.794367000 & $\mathrm{H}$ & 0.895304000 & 1.763590000 & 5.499328000 \\
\hline C & 2.473875000 & 1.857549000 & 2.525066000 & C & 2.186738000 & 0.574819000 & 4.250811000 \\
\hline $\mathrm{H}$ & 2.455359000 & 2.790827000 & 1.954874000 & $\mathrm{H}$ & 2.801208000 & 0.228828000 & 5.076044000 \\
\hline $\mathrm{H}$ & 1.467860000 & 1.426617000 & 2.509973000 & C & 2.473307000 & 0.149549000 & 2.952247000 \\
\hline \multirow[t]{8}{*}{$\mathrm{H}$} & 2.712332000 & 2.110688000 & 3.561458000 & $\mathrm{H}$ & 3.306299000 & -0.523136000 & 2.777144000 \\
\hline & & & & C & 1.679748000 & 0.590370000 & 1.882827000 \\
\hline & & & & C & 3.190462000 & -1.328320000 & 0.219157000 \\
\hline & & & & C & 4.577918000 & -1.133170000 & 0.136131000 \\
\hline & & & & $\mathrm{H}$ & 4.985209000 & -0.136118000 & 0.003867000 \\
\hline & & & & C & 5.439147000 & -2.228489000 & 0.215908000 \\
\hline & & & & $\mathrm{H}$ & 6.511350000 & -2.073936000 & 0.146894000 \\
\hline & & & & C & 4.925000000 & -3.516182000 & 0.378135000 \\
\hline
\end{tabular}




\begin{tabular}{|c|c|c|c|c|c|c|c|}
\hline & & & & $\mathrm{H}$ & 5.598523000 & -4.365675000 & 0.434909000 \\
\hline & & & & C & 3.544334000 & -3.714392000 & 0.461322000 \\
\hline & & & & $\mathrm{H}$ & 3.142778000 & -4.715484000 & 0.582831000 \\
\hline & & & & $\mathrm{H}$ & 1.604119000 & -2.785549000 & 0.435498000 \\
\hline & & & & C & 2.677602000 & -2.626192000 & 0.378481000 \\
\hline & & Int1 $\left(t \mathrm{Bu}_{3} \mathbf{P A u ^ { + }}\right)$ & & & & Int1 $\left(\operatorname{IPrAu} u^{+}\right)$ & \\
\hline C & 5.940301000 & -1.404669000 & 0.587636000 & C & 5.806054000 & -2.482389000 & 0.455490000 \\
\hline c & 5.874830000 & 1.222607000 & 0.534251000 & c & 6.302857000 & -0.282451000 & -0.893815000 \\
\hline C & 5.907671000 & -0.091032000 & 0.560144000 & C & 6.033077000 & -1.380928000 & -0.224801000 \\
\hline c & 1.978226000 & -0.036002000 & -1.337484000 & c & 1.927418000 & -1.099075000 & -1.738697000 \\
\hline C & 2.874834000 & 0.810765000 & -0.556014000 & c & 3.124282000 & -0.303513000 & -1.462852000 \\
\hline C & 4.126438000 & 1.214290000 & -1.381640000 & C & 4.263016000 & -0.644832000 & -2.458488000 \\
\hline c & 5.123766000 & 2.011387000 & -0.533145000 & c & 5.543760000 & 0.137928000 & -2.146702000 \\
\hline C & 6.646591000 & 2.064553000 & 1.532049000 & C & 7.445225000 & 0.633094000 & -0.497148000 \\
\hline C & 4.958855000 & -2.223410000 & 1.402652000 & C & 4.878379000 & -2.517023000 & 1.653973000 \\
\hline C & 6.987940000 & -2.191761000 & -0.175681000 & C & 6.477424000 & -3.793901000 & 0.098118000 \\
\hline H & 3.188830000 & 0.273967000 & 0.345318000 & H & 3.459379000 & -0.498636000 & -0.439019000 \\
\hline H & 2.340899000 & 1.713861000 & -0.237436000 & H & 2.875301000 & 0.761986000 & -1.528962000 \\
\hline $\mathrm{H}$ & 4.608117000 & 0.310644000 & -1.767183000 & $\mathrm{H}$ & 4.466459000 & -1.719344000 & -2.415416000 \\
\hline H & 3.806600000 & 1.811271000 & -2.241464000 & H & 3.926792000 & -0.412975000 & -3.474149000 \\
\hline $\mathrm{H}$ & 4.608925000 & 2.857786000 & -0.054809000 & $\mathrm{H}$ & 5.314256000 & 1.211835000 & -2.080839000 \\
\hline $\mathrm{H}$ & 5.862224000 & 2.468336000 & -1.206599000 & $\mathrm{H}$ & 6.223422000 & 0.042799000 & -3.005173000 \\
\hline H & 5.969236000 & 2.711121000 & 2.104429000 & H & 7.077958000 & 1.642526000 & -0.271588000 \\
\hline H & 7.205872000 & 1.443167000 & 2.233696000 & H & 7.975321000 & 0.255230000 & 0.378894000 \\
\hline $\mathrm{H}$ & 7.356764000 & 2.723157000 & 1.016090000 & $\mathrm{H}$ & 8.165531000 & 0.732432000 & -1.319029000 \\
\hline $\mathrm{H}$ & 4.392101000 & -2.907967000 & 0.758007000 & H & 4.061766000 & -3.233862000 & 1.496242000 \\
\hline $\mathrm{H}$ & 4.254433000 & -1.593216000 & 1.951882000 & $\mathrm{H}$ & 4.447246000 & -1.535469000 & 1.867369000 \\
\hline $\mathrm{H}$ & 5.492633000 & -2.847557000 & 2.130028000 & H & 5.420386000 & -2.850824000 & 2.547526000 \\
\hline H & 6.513992000 & -2.895590000 & -0.871618000 & $\mathrm{H}$ & 5.729568000 & -4.567969000 & -0.116527000 \\
\hline $\mathrm{H}$ & 7.597303000 & -2.789174000 & 0.513841000 & H & 7.078504000 & -4.158713000 & 0.940266000 \\
\hline $\mathrm{H}$ & 7.653880000 & -1.538591000 & -0.742618000 & $\mathrm{H}$ & 7.129808000 & -3.692172000 & -0.771282000 \\
\hline $\mathrm{H}$ & 1.012535000 & -1.428675000 & -2.847168000 & $\mathrm{H}$ & 0.420740000 & -2.630069000 & -2.495648000 \\
\hline $\mathrm{Au}$ & -0.460685000 & -0.231941000 & -0.708619000 & $\mathrm{Au}$ & -0.169163000 & -0.476210000 & -0.695529000 \\
\hline C & 1.289328000 & -0.765188000 & -2.052085000 & C & 0.997340000 & -1.843751000 & -2.051520000 \\
\hline$P$ & -2.579014000 & 0.054961000 & 0.304798000 & C & -1.579918000 & 0.492233000 & 0.385216000 \\
\hline C & -3.182199000 & 1.845858000 & -0.119843000 & N & -1.462763000 & 1.689152000 & 1.011669000 \\
\hline C & -2.372049000 & -0.188811000 & 2.213360000 & C & -2.659654000 & 2.023832000 & 1.631606000 \\
\hline C & -3.748720000 & -1.285436000 & -0.454636000 & $\mathrm{H}$ & -2.774777000 & 2.941045000 & 2.186349000 \\
\hline C & -3.577967000 & 0.337668000 & 3.020713000 & C & -3.530190000 & 1.011230000 & 1.380605000 \\
\hline $\mathrm{H}$ & -3.685445000 & 1.422090000 & 2.958686000 & H & -4.558503000 & 0.867235000 & 1.670519000 \\
\hline $\mathrm{H}$ & -4.521768000 & -0.120140000 & 2.718926000 & C & 0.639132000 & 2.323807000 & 2.111319000 \\
\hline $\mathrm{H}$ & -3.418844000 & 0.092126000 & 4.076655000 & C & -0.261827000 & 2.501400000 & 1.041623000 \\
\hline C & -5.048724000 & -1.477236000 & 0.354971000 & C & -0.060584000 & 3.441156000 & 0.010831000 \\
\hline H & -5.622919000 & -0.555126000 & 0.460971000 & C & -1.050231000 & 3.655605000 & -1.131165000 \\
\hline $\mathrm{H}$ & -5.682417000 & -2.195347000 & -0.177051000 & C & -0.409441000 & 3.359606000 & -2.501124000 \\
\hline $\mathrm{H}$ & -4.867879000 & -1.886840000 & 1.350248000 & $\mathrm{H}$ & -0.029804000 & 2.333225000 & -2.549337000 \\
\hline
\end{tabular}




\begin{tabular}{|c|c|c|c|c|c|c|c|}
\hline c & -2.397071000 & 2.868374000 & 0.729239000 & $\mathrm{H}$ & -1.146994000 & 3.485683000 & -3.300075000 \\
\hline $\mathrm{H}$ & -2.642842000 & 2.825327000 & 1.790630000 & $\mathrm{H}$ & 0.423958000 & 4.037572000 & -2.711269000 \\
\hline $\mathrm{H}$ & -1.315198000 & 2.755436000 & 0.608855000 & $\mathrm{H}$ & -1.878995000 & 2.952297000 & -1.005957000 \\
\hline $\mathrm{H}$ & -2.660741000 & 3.871840000 & 0.377925000 & $\mathrm{H}$ & -2.393744000 & 5.195779000 & -1.885560000 \\
\hline c & -2.850814000 & 2.162633000 & -1.597193000 & C & -1.648249000 & 5.076307000 & -1.093053000 \\
\hline $\mathrm{H}$ & -1.775146000 & 2.105323000 & -1.788747000 & $\mathrm{H}$ & -2.135909000 & 5.280796000 & -0.134776000 \\
\hline $\mathrm{H}$ & -3.360200000 & 1.519004000 & -2.311630000 & $\mathrm{H}$ & -0.878729000 & 5.840086000 & -1.243623000 \\
\hline $\mathrm{H}$ & -3.166984000 & 3.191583000 & -1.800768000 & $\mathrm{H}$ & 1.297679000 & 4.949418000 & -0.702537000 \\
\hline C & -4.693675000 & 2.042835000 & 0.123324000 & C & 1.107313000 & 4.212136000 & 0.070959000 \\
\hline $\mathrm{H}$ & -4.941360000 & 3.091026000 & -0.077653000 & C & 2.022013000 & 4.056728000 & 1.109032000 \\
\hline $\mathrm{H}$ & -5.309549000 & 1.437272000 & -0.544112000 & $\mathrm{H}$ & 2.917583000 & 4.670241000 & 1.137378000 \\
\hline $\mathrm{H}$ & -4.989266000 & 1.831937000 & 1.152733000 & C & 1.787724000 & 3.124971000 & 2.116781000 \\
\hline c & -1.082924000 & 0.526804000 & 2.682758000 & $\mathrm{H}$ & 2.504778000 & 3.021517000 & 2.925084000 \\
\hline H & -0.969297000 & 0.349363000 & 3.758031000 & $\mathrm{H}$ & -0.614312000 & 2.769771000 & 4.548571000 \\
\hline $\mathrm{H}$ & -0.195837000 & 0.118868000 & 2.188306000 & C & 0.210996000 & 2.052160000 & 4.591438000 \\
\hline $\mathrm{H}$ & -1.094793000 & 1.604211000 & 2.530308000 & $\mathrm{H}$ & 1.112179000 & 2.600923000 & 4.882952000 \\
\hline c & -2.171751000 & -1.687280000 & 2.532042000 & $\mathrm{H}$ & -0.008919000 & 1.329296000 & 5.383408000 \\
\hline $\mathrm{H}$ & -1.917807000 & -1.772006000 & 3.594188000 & C & 1.532499000 & 0.283847000 & 3.332900000 \\
\hline H & -3.068850000 & -2.286649000 & 2.371413000 & $\mathrm{H}$ & 1.644147000 & -0.265149000 & 2.391991000 \\
\hline $\mathrm{H}$ & -1.343909000 & -2.124791000 & 1.965956000 & $\mathrm{H}$ & 2.495004000 & 0.749467000 & 3.567714000 \\
\hline C & -4.110221000 & -0.897053000 & -1.904907000 & $\mathrm{H}$ & 1.315449000 & -0.440801000 & 4.124082000 \\
\hline $\mathrm{H}$ & -3.221467000 & -0.726629000 & -2.520114000 & C & 0.402831000 & 1.328257000 & 3.243538000 \\
\hline $\mathrm{H}$ & -4.658050000 & -1.734620000 & -2.349829000 & $\mathrm{H}$ & -0.522311000 & 0.782761000 & 3.033350000 \\
\hline $\mathrm{H}$ & -4.758457000 & -0.022284000 & -1.968659000 & $\mathrm{~N}$ & -2.850056000 & 0.076093000 & 0.612129000 \\
\hline C & -2.994758000 & -2.634059000 & -0.541615000 & C & -3.421636000 & -1.164243000 & 0.122261000 \\
\hline H & -2.700395000 & -3.037213000 & 0.425091000 & C & -4.068313000 & -1.154990000 & -1.130275000 \\
\hline $\mathrm{H}$ & -3.662274000 & -3.365013000 & -1.011096000 & C & -3.314714000 & -2.315996000 & 0.927630000 \\
\hline \multirow[t]{19}{*}{$\mathrm{H}$} & -2.100775000 & -2.557392000 & -1.168132000 & C & -2.641905000 & -2.318202000 & 2.297405000 \\
\hline & & & & $\mathrm{H}$ & -2.249545000 & -1.314769000 & 2.489684000 \\
\hline & & & & $\mathrm{H}$ & -0.702548000 & -3.041092000 & 1.577255000 \\
\hline & & & & C & -1.445374000 & -3.288144000 & 2.343050000 \\
\hline & & & & $\mathrm{H}$ & -1.759415000 & -4.324528000 & 2.183679000 \\
\hline & & & & $\mathrm{H}$ & -0.955348000 & -3.238736000 & 3.320733000 \\
\hline & & & & $\mathrm{H}$ & -3.168554000 & -2.584396000 & 4.394945000 \\
\hline & & & & C & -3.655404000 & -2.633749000 & 3.415821000 \\
\hline & & & & $\mathrm{H}$ & -4.488744000 & -1.924094000 & 3.414937000 \\
\hline & & & & $\mathrm{H}$ & -4.075354000 & -3.638351000 & 3.303990000 \\
\hline & & & & $\mathrm{H}$ & -3.814321000 & -4.406331000 & 1.016883000 \\
\hline & & & & C & -3.874628000 & -3.497681000 & 0.426296000 \\
\hline & & & & $\mathrm{H}$ & -4.941184000 & -4.453741000 & -1.177451000 \\
\hline & & & & C & -4.511771000 & -3.526027000 & -0.811338000 \\
\hline & & & & $\mathrm{H}$ & -5.115697000 & -2.401249000 & -2.536128000 \\
\hline & & & & C & -4.608394000 & -2.367293000 & -1.577179000 \\
\hline & & & & $\mathrm{H}$ & -3.716242000 & 0.927892000 & -1.470654000 \\
\hline & & & & C & -4.207613000 & 0.098786000 & -1.989244000 \\
\hline & & & & C & -5.687308000 & 0.490031000 & -2.174211000 \\
\hline
\end{tabular}




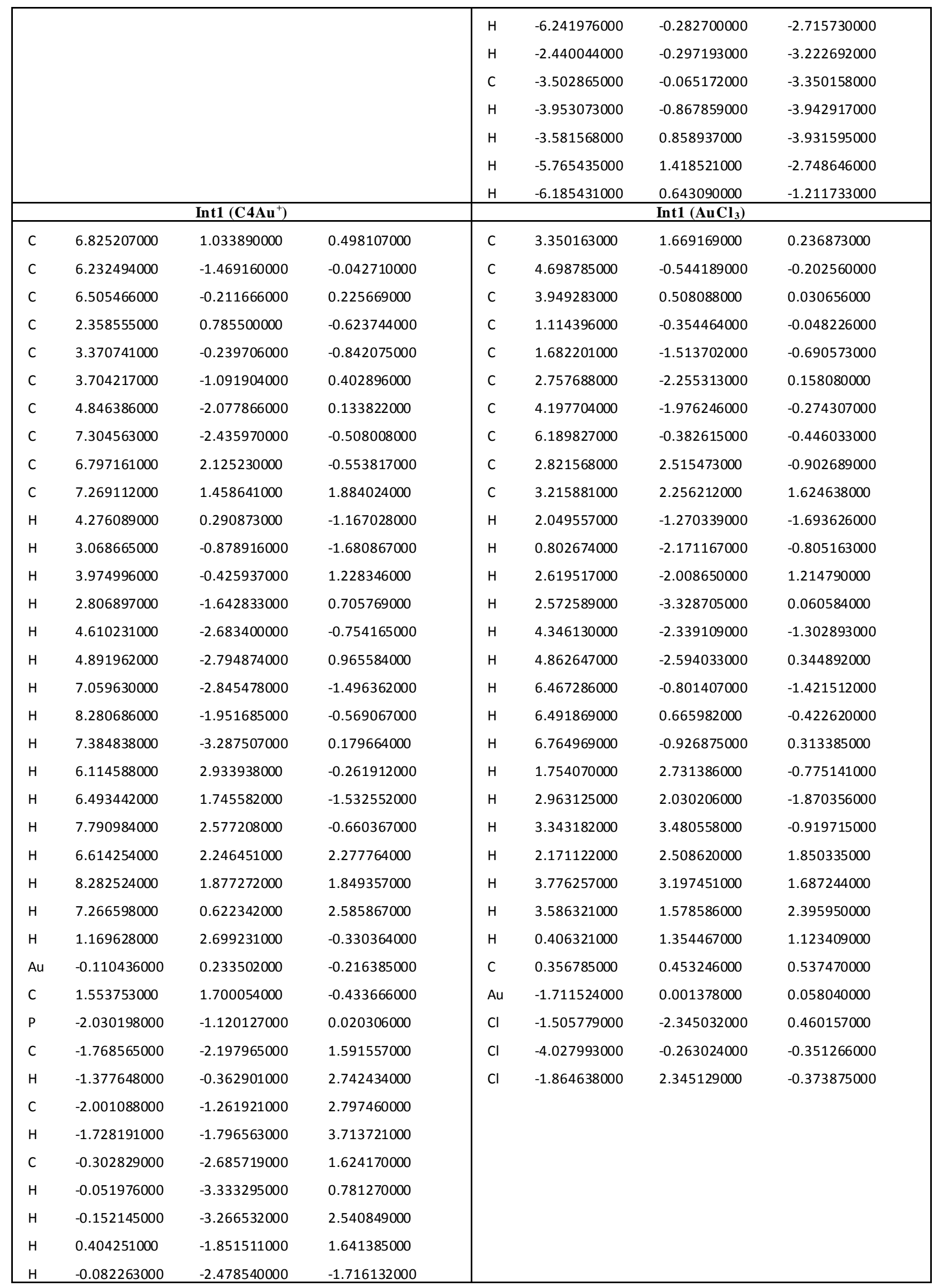




\begin{tabular}{|c|c|c|c|c|c|c|c|}
\hline c & -1.030963000 & -3.015553000 & -1.815861000 & & & & \\
\hline $\mathrm{H}$ & -1.032317000 & -3.854692000 & -1.115502000 & & & & \\
\hline $\mathrm{H}$ & -1.069249000 & -3.436079000 & -2.826600000 & & & & \\
\hline $\mathrm{H}$ & -2.526077000 & -4.149904000 & 0.899695000 & & & & \\
\hline c & -2.690220000 & -3.428629000 & 1.704270000 & & & & \\
\hline $\mathrm{H}$ & -2.462011000 & -3.941444000 & 2.645347000 & & & & \\
\hline $\mathrm{H}$ & -3.749762000 & -3.169312000 & 1.736957000 & & & & \\
\hline $\mathrm{H}$ & -3.632844000 & -3.665535000 & -0.922614000 & & & & \\
\hline C & -3.543328000 & -2.918301000 & -1.713529000 & & & & \\
\hline $\mathrm{H}$ & -3.533695000 & -3.456048000 & -2.668044000 & & & & \\
\hline $\mathrm{H}$ & -4.435040000 & -2.288849000 & -1.706872000 & & & & \\
\hline c & -2.247374000 & -2.089192000 & -1.618634000 & & & & \\
\hline C & -2.267892000 & -1.026993000 & -2.740009000 & & & & \\
\hline $\mathrm{H}$ & -2.393025000 & -1.534812000 & -3.702690000 & & & & \\
\hline $\mathrm{H}$ & -3.101840000 & -0.328404000 & -2.624854000 & & & & \\
\hline $\mathrm{H}$ & -1.337281000 & -0.453022000 & -2.782653000 & & & & \\
\hline C & -3.575502000 & -0.140026000 & 0.275811000 & & & & \\
\hline C & -4.765202000 & -0.862300000 & 0.507098000 & & & & \\
\hline H & -4.747689000 & -1.943463000 & 0.502691000 & & & & \\
\hline C & -5.981573000 & -0.228965000 & 0.741174000 & & & & \\
\hline $\mathrm{H}$ & -6.874270000 & -0.821351000 & 0.914811000 & & & & \\
\hline $\mathrm{H}$ & -6.975478000 & 1.677780000 & 0.930527000 & & & & \\
\hline C & -6.037042000 & 1.162961000 & 0.749904000 & & & & \\
\hline C & -4.875242000 & 1.894378000 & 0.524093000 & & & & \\
\hline $\mathrm{H}$ & -4.916193000 & 2.979028000 & 0.528547000 & & & & \\
\hline C & -3.633731000 & 1.276395000 & 0.285135000 & & & & \\
\hline C & -2.500695000 & 2.238009000 & 0.065842000 & & & & \\
\hline C & -1.889312000 & 2.862460000 & 1.167772000 & & & & \\
\hline $\mathrm{H}$ & -2.137395000 & 2.532814000 & 2.172477000 & & & & \\
\hline C & -1.007260000 & 3.929773000 & 0.983486000 & & & & \\
\hline $\mathrm{H}$ & -0.563059000 & 4.415085000 & 1.847512000 & & & & \\
\hline $\mathrm{H}$ & -0.070098000 & 5.248480000 & -0.446617000 & & & & \\
\hline C & -0.725407000 & 4.393810000 & -0.305153000 & & & & \\
\hline H & -1.111072000 & 4.132978000 & -2.412724000 & & & & \\
\hline C & -1.315193000 & 3.770892000 & -1.409527000 & & & & \\
\hline C & -2.196335000 & 2.702913000 & -1.226321000 & & & & \\
\hline $\mathrm{H}$ & -2.684855000 & 2.253174000 & -2.085659000 & & & & \\
\hline $\mathrm{H}$ & -3.044788000 & -0.952516000 & 2.887468000 & & & & \\
\hline \multicolumn{4}{|c|}{ TSa $\left(\mathrm{H}_{3} \mathrm{PAu}^{+}\right)$} & \multicolumn{4}{|c|}{$\mathbf{T S a}\left(\mathrm{Me}_{3} \mathrm{PAu}^{+}\right)$} \\
\hline C & -2.983202000 & 1.593077000 & 0.088294000 & C & 3.649545000 & -1.541700000 & 0.124411000 \\
\hline C & -4.159676000 & -0.739912000 & -0.184598000 & C & 4.575453000 & 0.876489000 & -0.261806000 \\
\hline C & -3.419355000 & 0.340219000 & -0.075321000 & C & 3.897231000 & -0.241940000 & -0.104209000 \\
\hline C & -0.967658000 & -0.155351000 & 0.248251000 & C & 1.617779000 & 0.080458000 & 0.270650000 \\
\hline C & -1.372003000 & -1.433191000 & 0.839340000 & C & 1.884293000 & 1.355910000 & 0.959207000 \\
\hline C & -2.136653000 & -2.359779000 & -0.138004000 & C & 2.507862000 & 2.416984000 & 0.026390000 \\
\hline C & -3.652750000 & -2.164768000 & -0.109040000 & C & 4.024629000 & 2.279397000 & -0.127326000 \\
\hline
\end{tabular}




\begin{tabular}{|c|c|c|c|c|c|c|c|}
\hline c & -5.663258000 & -0.597499000 & -0.350742000 & C & 6.065564000 & 0.798873000 & -0.546603000 \\
\hline c & -2.948404000 & 2.250221000 & 1.449870000 & C & 3.795618000 & -2.140791000 & 1.502769000 \\
\hline c & -2.616603000 & 2.460070000 & -1.088127000 & c & 3.272172000 & -2.483945000 & -0.984730000 \\
\hline $\mathrm{H}$ & -1.929128000 & -1.277721000 & 1.769698000 & $\mathrm{H}$ & 2.489022000 & 1.205261000 & 1.860056000 \\
\hline $\mathrm{H}$ & -0.420335000 & -1.904317000 & 1.125397000 & $\mathrm{H}$ & 0.896612000 & 1.695668000 & 1.298661000 \\
\hline $\mathrm{H}$ & -1.748942000 & -2.202115000 & -1.149514000 & $\mathrm{H}$ & 2.015132000 & 2.351671000 & -0.949163000 \\
\hline $\mathrm{H}$ & -1.913552000 & -3.397148000 & 0.128106000 & H & 2.284344000 & 3.410187000 & 0.427810000 \\
\hline $\mathrm{H}$ & -4.062053000 & -2.602993000 & 0.813200000 & $\mathrm{H}$ & 4.522789000 & 2.722700000 & 0.747575000 \\
\hline $\mathrm{H}$ & -4.104294000 & -2.739619000 & -0.926919000 & H & 4.362852000 & 2.876924000 & -0.982790000 \\
\hline $\mathrm{H}$ & -6.183609000 & -1.144758000 & 0.443964000 & $\mathrm{H}$ & 6.623095000 & 1.372968000 & 0.202669000 \\
\hline $\mathrm{H}$ & -5.983463000 & 0.445190000 & -0.321436000 & $\mathrm{H}$ & 6.432862000 & -0.228675000 & -0.542612000 \\
\hline $\mathrm{H}$ & -5.979456000 & -1.031856000 & -1.306173000 & $\mathrm{H}$ & 6.286535000 & 1.241833000 & -1.524663000 \\
\hline $\mathrm{H}$ & -1.954250000 & 2.657991000 & 1.664924000 & H & 2.857341000 & -2.605178000 & 1.827030000 \\
\hline $\mathrm{H}$ & -3.231850000 & 1.561628000 & 2.247208000 & H & 4.103572000 & -1.401343000 & 2.243156000 \\
\hline $\mathrm{H}$ & -3.646829000 & 3.096252000 & 1.459478000 & H & 4.548922000 & -2.937814000 & 1.471250000 \\
\hline $\mathrm{H}$ & -1.692353000 & 3.023418000 & -0.905151000 & $\mathrm{H}$ & 2.418841000 & -3.115894000 & -0.708696000 \\
\hline $\mathrm{H}$ & -3.402228000 & 3.214225000 & -1.225710000 & $\mathrm{H}$ & 4.113997000 & -3.168270000 & -1.153888000 \\
\hline $\mathrm{H}$ & -2.524560000 & 1.891179000 & -2.014512000 & $\mathrm{H}$ & 3.065734000 & -1.965462000 & -1.922023000 \\
\hline $\mathrm{H}$ & -0.248705000 & 1.593387000 & -0.729707000 & H & 0.933950000 & -1.608089000 & -0.823184000 \\
\hline $\mathrm{Au}$ & 1.914589000 & 0.091999000 & -0.062270000 & $\mathrm{Au}$ & -1.290647000 & -0.207112000 & -0.090845000 \\
\hline C & -0.096949000 & 0.643171000 & -0.233270000 & C & 0.753403000 & -0.697530000 & -0.266979000 \\
\hline$P$ & 4.223233000 & -0.394356000 & 0.059965000 & $\mathrm{P}$ & -3.611472000 & 0.209957000 & 0.028615000 \\
\hline $\mathrm{H}$ & 5.077106000 & 0.502843000 & -0.606047000 & C & -4.144068000 & 1.681377000 & -0.932215000 \\
\hline $\mathrm{H}$ & 4.800070000 & -0.428727000 & 1.342374000 & $\mathrm{H}$ & -5.226372000 & 1.821088000 & -0.852766000 \\
\hline \multirow[t]{11}{*}{$\mathrm{H}$} & 4.646499000 & -1.627019000 & -0.469241000 & $\mathrm{H}$ & -3.636491000 & 2.571605000 & -0.552974000 \\
\hline & & & & $\mathrm{H}$ & -3.873595000 & 1.549897000 & -1.982704000 \\
\hline & & & & C & -4.220782000 & 0.495058000 & 1.737301000 \\
\hline & & & & $\mathrm{H}$ & -3.718184000 & 1.365119000 & 2.166622000 \\
\hline & & & & $\mathrm{H}$ & -5.301443000 & 0.666533000 & 1.736420000 \\
\hline & & & & $\mathrm{H}$ & -3.994312000 & -0.376300000 & 2.356489000 \\
\hline & & & & C & -4.616614000 & -1.182840000 & -0.619652000 \\
\hline & & & & H & -5.683753000 & -0.951461000 & -0.549837000 \\
\hline & & & & H & -4.355674000 & -1.368259000 & -1.664444000 \\
\hline & & & & $\mathrm{H}$ & -4.405044000 & -2.087028000 & -0.043702000 \\
\hline & \multicolumn{3}{|c|}{$\mathbf{T S a}\left(\mathbf{E t}_{3} \mathbf{P A u}^{+}\right)$} & \multicolumn{4}{|c|}{ TSa $\left(\mathbf{P h}_{3} \mathbf{P A u}^{+}\right)$} \\
\hline C & -4.175814000 & 1.476742000 & -0.133877000 & C & -5.270309000 & 1.579653000 & 0.072754000 \\
\hline C & -5.009602000 & -0.995584000 & 0.042840000 & C & -6.095854000 & -0.875447000 & -0.276343000 \\
\hline C & -4.363047000 & 0.147670000 & -0.068963000 & C & -5.444221000 & 0.262615000 & -0.136586000 \\
\hline C & -2.096783000 & -0.019890000 & 0.298037000 & c & -3.211393000 & 0.011938000 & 0.239086000 \\
\hline C & -2.290428000 & -1.110703000 & 1.272313000 & C & -3.420108000 & -1.248264000 & 0.979357000 \\
\hline C & -2.894656000 & -2.378148000 & 0.630925000 & C & -4.001882000 & -2.363311000 & 0.085321000 \\
\hline C & -4.416986000 & -2.317521000 & 0.478636000 & C & -5.518204000 & -2.262833000 & -0.102655000 \\
\hline C & -6.505154000 & -1.022189000 & -0.221566000 & C & -7.583045000 & -0.837107000 & -0.582346000 \\
\hline C & -4.333860000 & 2.352196000 & 1.086050000 & C & -5.464841000 & 2.190693000 & 1.439016000 \\
\hline C & -3.849897000 & 2.171459000 & -1.425958000 & C & -4.908771000 & 2.519743000 & -1.042128000 \\
\hline $\mathrm{H}$ & -2.877323000 & -0.778053000 & 2.135464000 & $\mathrm{H}$ & -4.031053000 & -1.090923000 & 1.874906000 \\
\hline
\end{tabular}




\begin{tabular}{|c|c|c|c|c|c|c|c|}
\hline H & -1.280962000 & -1.329713000 & 1.644529000 & H & -2.419182000 & -1.535269000 & 1.327143000 \\
\hline $\mathrm{H}$ & -2.417191000 & -2.533822000 & -0.342037000 & $\mathrm{H}$ & -3.491822000 & -2.328605000 & -0.882913000 \\
\hline $\mathrm{H}$ & -2.637200000 & -3.242006000 & 1.251523000 & $\mathrm{H}$ & -3.765492000 & -3.333457000 & 0.533319000 \\
\hline $\mathrm{H}$ & -4.891254000 & -2.562231000 & 1.440573000 & $\mathrm{H}$ & -6.024850000 & -2.696622000 & 0.772211000 \\
\hline $\mathrm{H}$ & -4.749973000 & -3.101733000 & -0.212244000 & $\mathrm{H}$ & -5.827945000 & -2.886094000 & -0.950607000 \\
\hline $\mathrm{H}$ & -7.034917000 & -1.430299000 & 0.647216000 & $\mathrm{H}$ & -8.138053000 & -1.410828000 & 0.169165000 \\
\hline $\mathrm{H}$ & -6.903227000 & -0.029388000 & -0.437840000 & H & -7.973623000 & 0.181729000 & -0.601944000 \\
\hline $\mathrm{H}$ & -6.726686000 & -1.676793000 & -1.072519000 & $\mathrm{H}$ & -7.781360000 & -1.301921000 & -1.555192000 \\
\hline $\mathrm{H}$ & -3.421301000 & 2.929402000 & 1.274192000 & H & -4.547418000 & 2.686243000 & 1.777099000 \\
\hline $\mathrm{H}$ & -4.582559000 & 1.775047000 & 1.977455000 & $\mathrm{H}$ & -5.766867000 & 1.451431000 & 2.181958000 \\
\hline $\mathrm{H}$ & -5.135776000 & 3.079119000 & 0.905766000 & $\mathrm{H}$ & -6.239347000 & 2.965636000 & 1.380881000 \\
\hline $\mathrm{H}$ & -3.009200000 & 2.867264000 & -1.312627000 & $\mathrm{H}$ & -4.079232000 & 3.181258000 & -0.763182000 \\
\hline $\mathrm{H}$ & -4.714786000 & 2.784363000 & -1.712099000 & H & -5.770632000 & 3.173923000 & -1.229262000 \\
\hline $\mathrm{H}$ & -3.648643000 & 1.471641000 & -2.238125000 & H & -4.674825000 & 1.996848000 & -1.970319000 \\
\hline $\mathrm{H}$ & -1.488725000 & 1.386750000 & -1.174248000 & H & -2.563485000 & 1.671689000 & -0.918719000 \\
\hline $\mathrm{Au}$ & 0.800373000 & 0.250748000 & -0.219107000 & $\mathrm{Au}$ & -0.307447000 & 0.339751000 & -0.150662000 \\
\hline c & -1.267890000 & 0.632179000 & -0.430637000 & C & -2.361984000 & 0.785413000 & -0.331817000 \\
\hline$P$ & 3.142942000 & -0.038876000 & -0.096125000 & $P$ & 2.028769000 & -0.032775000 & -0.007311000 \\
\hline C & 3.843177000 & -0.916216000 & -1.565836000 & C & 2.964392000 & 1.533248000 & -0.148089000 \\
\hline $\mathrm{H}$ & 4.931596000 & -0.961602000 & -1.444846000 & $\mathrm{H}$ & 1.663931000 & 2.296790000 & -1.699824000 \\
\hline $\mathrm{H}$ & 3.645424000 & -0.267461000 & -2.426323000 & C & 2.542531000 & 2.487099000 & -1.089086000 \\
\hline C & 3.651861000 & -0.994990000 & 1.407085000 & C & 3.248291000 & 3.678869000 & -1.243110000 \\
\hline $\mathrm{H}$ & 3.115179000 & -1.948701000 & 1.360053000 & C & 4.373513000 & 3.932293000 & -0.454359000 \\
\hline $\mathrm{H}$ & 3.233365000 & -0.456238000 & 2.264121000 & $\mathrm{H}$ & 2.917293000 & 4.411295000 & -1.972902000 \\
\hline C & 4.046824000 & 1.574393000 & -0.040620000 & $\mathrm{H}$ & 4.918568000 & 4.864055000 & -0.570366000 \\
\hline H & 5.121680000 & 1.360451000 & -0.049377000 & C & 4.794066000 & 2.990420000 & 0.485776000 \\
\hline $\mathrm{H}$ & 3.816202000 & 2.083362000 & -0.983001000 & $\mathrm{H}$ & 5.666033000 & 3.186748000 & 1.102024000 \\
\hline C & 5.159578000 & -1.220927000 & 1.583345000 & C & 4.094667000 & 1.792119000 & 0.641560000 \\
\hline $\mathrm{H}$ & 5.586697000 & -1.798740000 & 0.758834000 & $\mathrm{H}$ & 4.426201000 & 1.067371000 & 1.377949000 \\
\hline $\mathrm{H}$ & 5.708909000 & -0.278180000 & 1.659299000 & C & 1.861525000 & -0.347022000 & 2.760647000 \\
\hline H & 5.342551000 & -1.781545000 & 2.504571000 & H & 1.090775000 & 0.417167000 & 2.703001000 \\
\hline C & 3.672174000 & 2.460585000 & 1.154284000 & C & 2.216305000 & -0.881486000 & 3.998015000 \\
\hline $\mathrm{H}$ & 3.938315000 & 1.994620000 & 2.107653000 & H & 1.722373000 & -0.530432000 & 4.898859000 \\
\hline $\mathrm{H}$ & 4.206046000 & 3.412965000 & 1.094332000 & C & 3.200166000 & -1.870760000 & 4.076609000 \\
\hline $\mathrm{H}$ & 2.599380000 & 2.677264000 & 1.169927000 & $\mathrm{H}$ & 3.471342000 & -2.290928000 & 5.040292000 \\
\hline C & 3.257742000 & -2.314258000 & -1.802953000 & C & 3.830774000 & -2.321888000 & 2.916222000 \\
\hline $\mathrm{H}$ & 3.473740000 & -2.994233000 & -0.973526000 & $\mathrm{H}$ & 4.592847000 & -3.092942000 & 2.974279000 \\
\hline $\mathrm{H}$ & 2.172276000 & -2.274879000 & -1.937342000 & C & 3.484395000 & -1.786390000 & 1.674138000 \\
\hline \multirow[t]{8}{*}{$\mathrm{H}$} & 3.691326000 & -2.751383000 & -2.706636000 & $\mathrm{H}$ & 3.977957000 & -2.145594000 & 0.777066000 \\
\hline & & & & C & 2.498172000 & -0.792362000 & 1.590078000 \\
\hline & & & & C & 2.638635000 & -1.137859000 & -1.331108000 \\
\hline & & & & C & 3.910580000 & -0.966657000 & -1.899127000 \\
\hline & & & & $\mathrm{H}$ & 4.547000000 & -0.148046000 & -1.578848000 \\
\hline & & & & C & 4.357903000 & -1.846682000 & -2.885708000 \\
\hline & & & & $\mathrm{H}$ & 5.341392000 & -1.707294000 & -3.323785000 \\
\hline & & & & C & 3.543496000 & -2.897944000 & -3.310652000 \\
\hline
\end{tabular}




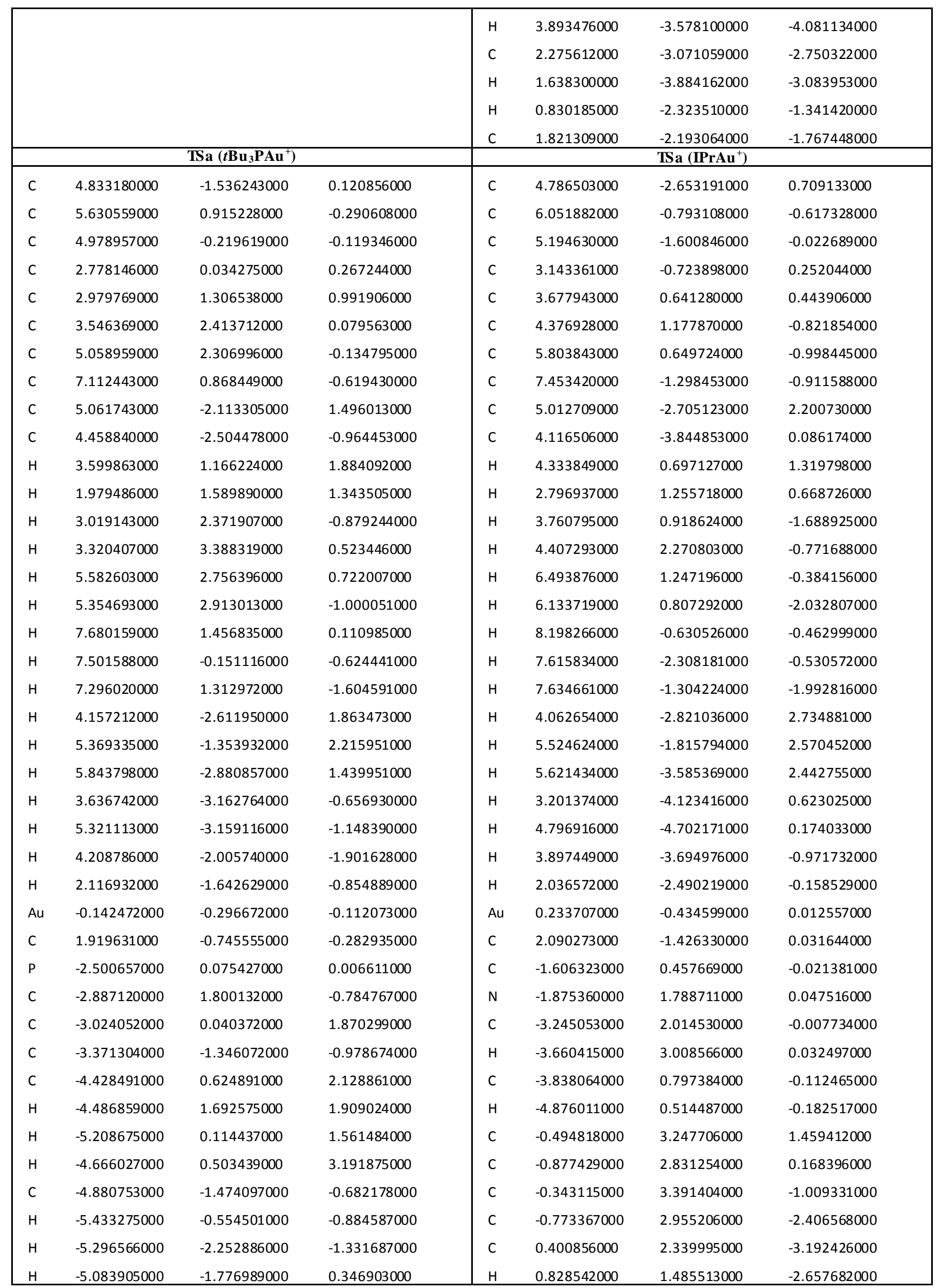




\begin{tabular}{|c|c|c|c|c|c|c|c|}
\hline c & -2.437808000 & 2.924534000 & 0.174202000 & $\mathrm{H}$ & 0.060290000 & 1.992400000 & -4.173180000 \\
\hline $\mathrm{H}$ & -3.059431000 & 3.004298000 & 1.066987000 & $\mathrm{H}$ & 1.199159000 & 3.070664000 & -3.360010000 \\
\hline $\mathrm{H}$ & -1.392736000 & 2.811244000 & 0.477833000 & $\mathrm{H}$ & -1.532492000 & 2.174603000 & -2.301842000 \\
\hline $\mathrm{H}$ & -2.520764000 & 3.877557000 & -0.359842000 & $\mathrm{H}$ & -1.770936000 & 3.768222000 & -4.163356000 \\
\hline c & -2.036608000 & 1.966694000 & -2.067069000 & C & -1.418184000 & 4.117148000 & -3.187600000 \\
\hline $\mathrm{H}$ & -0.966153000 & 1.899769000 & -1.850973000 & $\mathrm{H}$ & -2.272863000 & 4.537385000 & -2.648371000 \\
\hline $\mathrm{H}$ & -2.271277000 & 1.244784000 & -2.846766000 & $\mathrm{H}$ & -0.705222000 & 4.928772000 & -3.364704000 \\
\hline $\mathrm{H}$ & -2.230805000 & 2.964417000 & -2.476991000 & $\mathrm{H}$ & 1.054748000 & 4.853882000 & -1.743197000 \\
\hline C & -4.377346000 & 2.003934000 & -1.128312000 & C & 0.622068000 & 4.395412000 & -0.859412000 \\
\hline $\mathrm{H}$ & -4.509393000 & 3.022373000 & -1.511177000 & C & 1.030480000 & 4.820988000 & 0.401922000 \\
\hline $\mathrm{H}$ & -4.725365000 & 1.323277000 & -1.907152000 & $\mathrm{H}$ & 1.776090000 & 5.605445000 & 0.493414000 \\
\hline $\mathrm{H}$ & -5.030970000 & 1.896418000 & -0.260641000 & C & 0.476080000 & 4.253621000 & 1.546271000 \\
\hline c & -1.980401000 & 0.823708000 & 2.702466000 & $\mathrm{H}$ & 0.794201000 & 4.601510000 & 2.524177000 \\
\hline H & -2.267342000 & 0.757364000 & 3.758098000 & $\mathrm{H}$ & -2.611363000 & 4.231452000 & 2.960924000 \\
\hline $\mathrm{H}$ & -0.982080000 & 0.386826000 & 2.603073000 & C & -1.833087000 & 3.740474000 & 3.553472000 \\
\hline $\mathrm{H}$ & -1.919997000 & 1.880290000 & 2.448435000 & $\mathrm{H}$ & -1.147560000 & 4.516446000 & 3.909208000 \\
\hline c & -2.981770000 & -1.411798000 & 2.394297000 & $\mathrm{H}$ & -2.308200000 & 3.290955000 & 4.431264000 \\
\hline $\mathrm{H}$ & -3.137917000 & -1.384358000 & 3.478331000 & C & -0.025552000 & 1.953281000 & 3.588850000 \\
\hline H & -3.765806000 & -2.043448000 & 1.975103000 & $\mathrm{H}$ & 0.461729000 & 1.150187000 & 3.026205000 \\
\hline $\mathrm{H}$ & -2.010884000 & -1.884026000 & 2.216868000 & $\mathrm{H}$ & 0.748606000 & 2.651330000 & 3.924556000 \\
\hline C & -3.176589000 & -1.120799000 & -2.494273000 & $\mathrm{H}$ & -0.482986000 & 1.513450000 & 4.481043000 \\
\hline $\mathrm{H}$ & -2.122478000 & -0.995493000 & -2.758773000 & C & -1.094360000 & 2.663014000 & 2.734963000 \\
\hline $\mathrm{H}$ & -3.540551000 & -2.011888000 & -3.017425000 & $\mathrm{H}$ & -1.833529000 & 1.908034000 & 2.451604000 \\
\hline $\mathrm{H}$ & -3.742275000 & -0.271570000 & -2.879560000 & $\mathrm{~N}$ & -2.819231000 & -0.146849000 & -0.119117000 \\
\hline C & -2.668772000 & -2.686435000 & -0.652979000 & C & -3.035240000 & -1.576462000 & -0.215827000 \\
\hline H & -2.749973000 & -2.983436000 & 0.390551000 & C & -3.088964000 & -2.161989000 & -1.496404000 \\
\hline $\mathrm{H}$ & -3.139128000 & -3.472117000 & -1.255081000 & C & -3.205605000 & -2.312323000 & 0.973951000 \\
\hline \multirow[t]{19}{*}{$\mathrm{H}$} & -1.607590000 & -2.656498000 & -0.917889000 & C & -3.169430000 & -1.675004000 & 2.359970000 \\
\hline & & & & $\mathrm{H}$ & -2.975798000 & -0.604810000 & 2.239833000 \\
\hline & & & & $\mathrm{H}$ & -1.055364000 & -2.110877000 & 2.725679000 \\
\hline & & & & C & -2.024170000 & -2.248792000 & 3.216711000 \\
\hline & & & & $\mathrm{H}$ & -2.158630000 & -3.319360000 & 3.402611000 \\
\hline & & & & $\mathrm{H}$ & -1.989625000 & -1.746312000 & 4.188950000 \\
\hline & & & & $\mathrm{H}$ & -4.497741000 & -1.309197000 & 4.048260000 \\
\hline & & & & C & -4.525912000 & -1.816430000 & 3.078476000 \\
\hline & & & & $\mathrm{H}$ & -5.339138000 & -1.379107000 & 2.490634000 \\
\hline & & & & $\mathrm{H}$ & -4.775632000 & -2.866570000 & 3.260814000 \\
\hline & & & & $\mathrm{H}$ & -3.562048000 & -4.290300000 & 1.741616000 \\
\hline & & & & C & -3.424829000 & -3.689744000 & 0.847816000 \\
\hline & & & & $\mathrm{H}$ & -3.648748000 & -5.369864000 & -0.475657000 \\
\hline & & & & C & -3.474549000 & -4.300464000 & -0.402246000 \\
\hline & & & & $\mathrm{H}$ & -3.356548000 & -4.031080000 & -2.527533000 \\
\hline & & & & C & -3.309343000 & -3.543480000 & -1.558845000 \\
\hline & & & & $\mathrm{H}$ & -2.779865000 & -0.310766000 & -2.520937000 \\
\hline & & & & C & -2.923449000 & -1.362728000 & -2.785563000 \\
\hline & & & & C & -4.186420000 & -1.441806000 & -3.665980000 \\
\hline
\end{tabular}




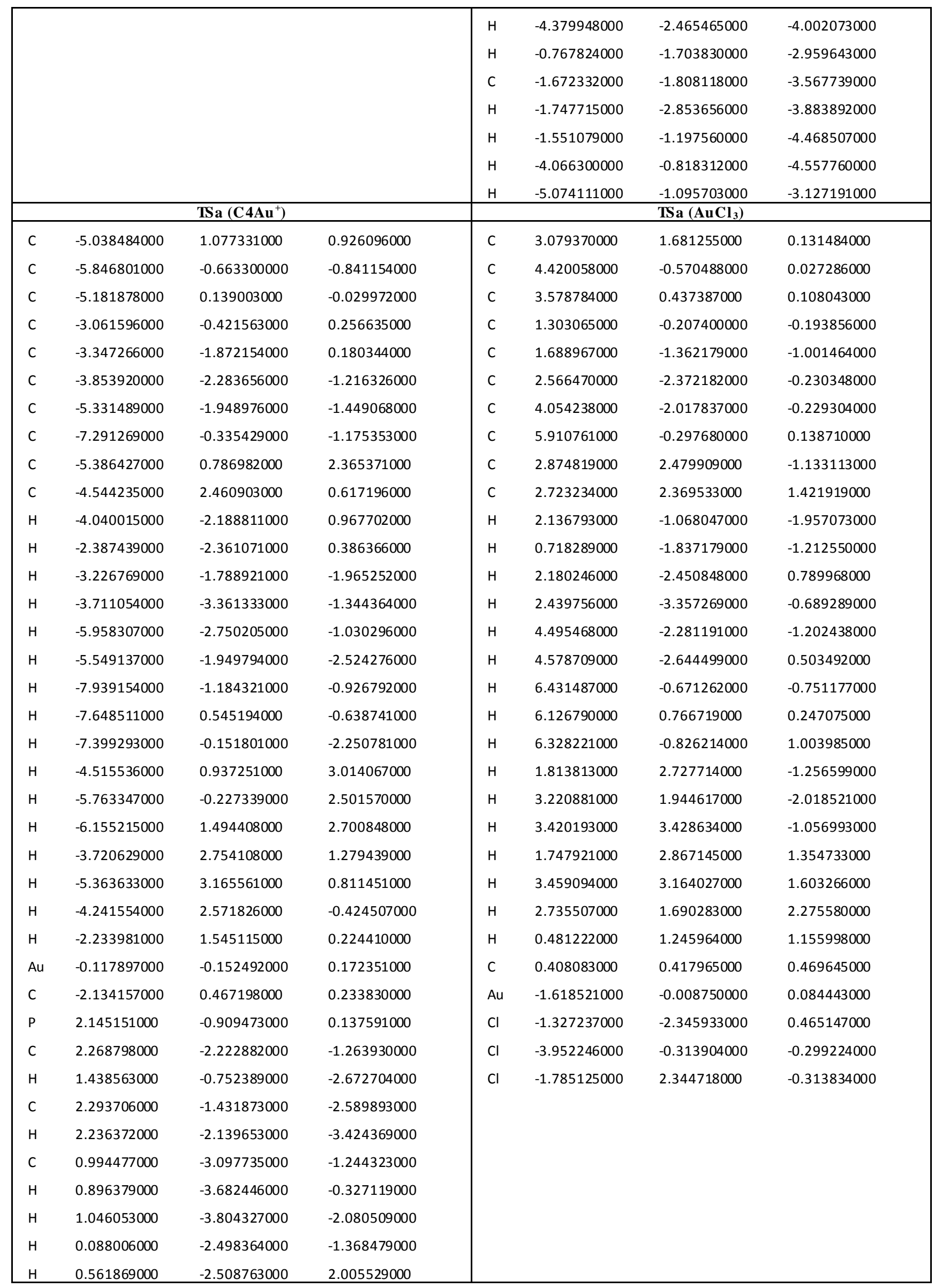




\begin{tabular}{|c|c|c|c|c|c|c|c|}
\hline c & 1.610536000 & -2.752727000 & 2.203509000 & & & & \\
\hline $\mathrm{H}$ & 1.875063000 & -3.648320000 & 1.634943000 & & & & \\
\hline H & 1.699340000 & -3.005221000 & 3.266016000 & & & & \\
\hline $\mathrm{H}$ & 3.495469000 & -3.773649000 & -0.293760000 & & & & \\
\hline c & 3.492148000 & -3.157597000 & -1.196390000 & & & & \\
\hline $\mathrm{H}$ & 3.451869000 & -3.841411000 & -2.051948000 & & & & \\
\hline $\mathrm{H}$ & 4.441835000 & -2.624389000 & -1.263859000 & & & & \\
\hline $\mathrm{H}$ & 4.335737000 & -2.793104000 & 1.450389000 & & & & \\
\hline C & 4.008739000 & -2.000010000 & 2.126296000 & & & & \\
\hline $\mathrm{H}$ & 4.090747000 & -2.390326000 & 3.147057000 & & & & \\
\hline $\mathrm{H}$ & 4.701670000 & -1.160577000 & 2.045178000 & & & & \\
\hline c & 2.547776000 & -1.567938000 & 1.893665000 & & & & \\
\hline C & 2.229030000 & -0.404662000 & 2.859630000 & & & & \\
\hline $\mathrm{H}$ & 2.442423000 & -0.728618000 & 3.884590000 & & & & \\
\hline $\mathrm{H}$ & 2.846914000 & 0.475140000 & 2.656427000 & & & & \\
\hline $\mathrm{H}$ & 1.176931000 & -0.108105000 & 2.812615000 & & & & \\
\hline C & 3.411048000 & 0.390195000 & -0.236467000 & & & & \\
\hline C & 4.761021000 & -0.008891000 & -0.331185000 & & & & \\
\hline H & 5.028158000 & -1.045164000 & -0.177518000 & & & & \\
\hline C & 5.782698000 & 0.892903000 & -0.613328000 & & & & \\
\hline $\mathrm{H}$ & 6.808290000 & 0.543262000 & -0.678303000 & & & & \\
\hline $\mathrm{H}$ & 6.257547000 & 2.958122000 & -1.025703000 & & & & \\
\hline C & 5.475278000 & 2.237830000 & -0.807067000 & & & & \\
\hline C & 4.150441000 & 2.653261000 & -0.720794000 & & & & \\
\hline $\mathrm{H}$ & 3.904780000 & 3.699261000 & -0.875513000 & & & & \\
\hline C & 3.101319000 & 1.758006000 & -0.442730000 & & & & \\
\hline C & 1.738232000 & 2.385409000 & -0.409872000 & & & & \\
\hline C & 1.004567000 & 2.542881000 & -1.597418000 & & & & \\
\hline $\mathrm{H}$ & 1.378200000 & 2.103568000 & -2.517738000 & & & & \\
\hline C & -0.176503000 & 3.287645000 & -1.611024000 & & & & \\
\hline $\mathrm{H}$ & -0.720229000 & 3.415475000 & -2.542489000 & & & & \\
\hline $\mathrm{H}$ & -1.539518000 & 4.498582000 & -0.457032000 & & & & \\
\hline C & -0.639220000 & 3.890868000 & -0.438745000 & & & & \\
\hline H & -0.262725000 & 4.216449000 & 1.661594000 & & & & \\
\hline C & 0.079374000 & 3.736159000 & 0.749348000 & & & & \\
\hline C & 1.261594000 & 2.992483000 & 0.762813000 & & & & \\
\hline $\mathrm{H}$ & 1.834889000 & 2.905205000 & 1.680881000 & & & & \\
\hline $\mathrm{H}$ & 3.210348000 & -0.849135000 & -2.706760000 & & & & \\
\hline \multicolumn{4}{|c|}{ Int2 $\left(\mathrm{H}_{3} \mathrm{PAu}^{+}\right)$} & \multicolumn{4}{|c|}{ Int2 $\left(\mathrm{Me}_{3} \mathrm{PAu}^{+}\right)$} \\
\hline C & 3.035774000 & -1.533871000 & 0.159565000 & C & 3.652646000 & -1.510119000 & 0.157439000 \\
\hline C & 3.594741000 & 0.842437000 & -0.225642000 & C & 4.184171000 & 0.870313000 & -0.224750000 \\
\hline C & 2.649128000 & -0.175826000 & 0.019539000 & C & 3.248146000 & -0.156541000 & 0.017837000 \\
\hline C & 1.229047000 & 0.221252000 & 0.294996000 & C & 1.824095000 & 0.219906000 & 0.292093000 \\
\hline C & 1.063669000 & 1.533169000 & 1.024305000 & C & 1.637985000 & 1.534520000 & 1.012632000 \\
\hline C & 1.742395000 & 2.624933000 & 0.188172000 & C & 2.310862000 & 2.631551000 & 0.178953000 \\
\hline C & 3.216547000 & 2.282998000 & -0.038585000 & C & 3.791088000 & 2.306760000 & -0.033169000 \\
\hline
\end{tabular}




\begin{tabular}{|c|c|c|c|c|c|c|c|}
\hline c & 5.044353000 & 0.669520000 & -0.572723000 & C & 5.636092000 & 0.713471000 & -0.571873000 \\
\hline c & 2.243681000 & -2.474974000 & 1.006311000 & C & 2.881924000 & -2.456805000 & 1.017655000 \\
\hline c & 4.231263000 & -2.171218000 & -0.472754000 & c & 4.846958000 & -2.135420000 & -0.489372000 \\
\hline $\mathrm{H}$ & 1.523124000 & 1.488935000 & 2.022555000 & $\mathrm{H}$ & 2.088047000 & 1.502563000 & 2.016037000 \\
\hline $\mathrm{H}$ & 0.002754000 & 1.753381000 & 1.160632000 & $\mathrm{H}$ & 0.572744000 & 1.740481000 & 1.136643000 \\
\hline $\mathrm{H}$ & 1.229291000 & 2.703401000 & -0.776266000 & $\mathrm{H}$ & 1.806087000 & 2.699222000 & -0.790799000 \\
\hline $\mathrm{H}$ & 1.672918000 & 3.604099000 & 0.671890000 & H & 2.225692000 & 3.612393000 & 0.657194000 \\
\hline $\mathrm{H}$ & 3.825885000 & 2.613484000 & 0.822133000 & $\mathrm{H}$ & 4.386720000 & 2.637891000 & 0.836887000 \\
\hline $\mathrm{H}$ & 3.643368000 & 2.846648000 & -0.881432000 & H & 4.221145000 & 2.879813000 & -0.867886000 \\
\hline $\mathrm{H}$ & 5.572754000 & 1.619815000 & -0.477959000 & $\mathrm{H}$ & 6.156499000 & 1.666839000 & -0.463223000 \\
\hline $\mathrm{H}$ & 5.557870000 & -0.071534000 & 0.043019000 & $\mathrm{H}$ & 6.155717000 & -0.031795000 & 0.033246000 \\
\hline $\mathrm{H}$ & 5.145665000 & 0.345651000 & -1.616826000 & $\mathrm{H}$ & 5.740995000 & 0.406341000 & -1.620762000 \\
\hline $\mathrm{H}$ & 1.697234000 & -3.183500000 & 0.368231000 & H & 2.337766000 & -3.176647000 & 0.390773000 \\
\hline $\mathrm{H}$ & 1.517530000 & -1.975421000 & 1.644974000 & H & 2.155299000 & -1.960643000 & 1.658592000 \\
\hline $\mathrm{H}$ & 2.936647000 & -3.088300000 & 1.596697000 & H & 3.589089000 & -3.056231000 & 1.605827000 \\
\hline $\mathrm{H}$ & 4.027131000 & -3.232438000 & -0.642981000 & $\mathrm{H}$ & 4.645142000 & -3.195597000 & -0.669877000 \\
\hline $\mathrm{H}$ & 5.087873000 & -2.142172000 & 0.218513000 & $\mathrm{H}$ & 5.708129000 & -2.111390000 & 0.196333000 \\
\hline $\mathrm{H}$ & 4.536845000 & -1.712527000 & -1.411130000 & $\mathrm{H}$ & 5.143901000 & -1.666025000 & -1.425181000 \\
\hline $\mathrm{H}$ & 0.497435000 & -1.363164000 & -0.831818000 & $\mathrm{H}$ & 1.122535000 & -1.381966000 & -0.818860000 \\
\hline $\mathrm{Au}$ & -1.786818000 & -0.148435000 & -0.110920000 & $\mathrm{Au}$ & -1.204326000 & -0.202848000 & -0.112343000 \\
\hline C & 0.214561000 & -0.492507000 & -0.235707000 & C & 0.816354000 & -0.512197000 & -0.230519000 \\
\hline$P$ & -4.164039000 & 0.183379000 & -0.006396000 & $\mathrm{P}$ & -3.580548000 & 0.091465000 & -0.014424000 \\
\hline $\mathrm{H}$ & -4.980737000 & -0.737613000 & -0.691319000 & C & -4.218229000 & 1.458772000 & -1.068297000 \\
\hline $\mathrm{H}$ & -4.778240000 & 0.159103000 & 1.261267000 & $\mathrm{H}$ & -5.306091000 & 1.542252000 & -0.986397000 \\
\hline \multirow[t]{11}{*}{$\mathrm{H}$} & -4.687100000 & 1.392355000 & -0.506407000 & $\mathrm{H}$ & -3.760706000 & 2.402076000 & -0.759631000 \\
\hline & & & & $\mathrm{H}$ & -3.949027000 & 1.272239000 & -2.110963000 \\
\hline & & & & C & -4.229938000 & 0.469359000 & 1.665473000 \\
\hline & & & & $\mathrm{H}$ & -3.776457000 & 1.393001000 & 2.033747000 \\
\hline & & & & $\mathrm{H}$ & -5.317960000 & 0.583985000 & 1.648576000 \\
\hline & & & & $\mathrm{H}$ & -3.963583000 & -0.339822000 & 2.350058000 \\
\hline & & & & C & -4.540442000 & -1.383667000 & -0.552291000 \\
\hline & & & & $\mathrm{H}$ & -5.616512000 & -1.194289000 & -0.492253000 \\
\hline & & & & H & -4.276305000 & -1.635806000 & -1.582376000 \\
\hline & & & & $\mathrm{H}$ & -4.289162000 & -2.235313000 & 0.084887000 \\
\hline & \multicolumn{3}{|c|}{ Int2 $\left(\mathrm{Et}_{3} \mathrm{PAu}^{+}\right)$} & \multicolumn{4}{|c|}{ Int2 $\left(\mathrm{Ph}_{3} \mathrm{PAu}^{+}\right)$} \\
\hline C & -4.162667000 & 1.462478000 & -0.072118000 & C & -5.250300000 & 1.553689000 & 0.115747000 \\
\hline C & -4.649079000 & -0.954590000 & 0.053504000 & C & -5.770011000 & -0.832733000 & -0.242762000 \\
\hline C & -3.730789000 & 0.115539000 & 0.050230000 & C & -4.838886000 & 0.200726000 & -0.011923000 \\
\hline C & -2.291252000 & -0.171990000 & 0.348179000 & c & -3.413248000 & -0.163760000 & 0.264063000 \\
\hline C & -2.052496000 & -1.309403000 & 1.313700000 & C & -3.215710000 & -1.470778000 & 0.995502000 \\
\hline C & -2.723250000 & -2.564546000 & 0.742564000 & C & -3.885623000 & -2.578846000 & 0.174081000 \\
\hline C & -4.216397000 & -2.314799000 & 0.519393000 & C & -5.368709000 & -2.264953000 & -0.035775000 \\
\hline C & -6.113995000 & -0.896572000 & -0.269175000 & C & -7.223069000 & -0.686658000 & -0.590481000 \\
\hline C & -3.389334000 & 2.575973000 & 0.554541000 & C & -4.487495000 & 2.510131000 & 0.972365000 \\
\hline C & -5.388954000 & 1.924998000 & -0.791779000 & C & -6.444676000 & 2.167943000 & -0.541613000 \\
\hline $\mathrm{H}$ & -2.473710000 & -1.083144000 & 2.304668000 & $\mathrm{H}$ & -3.661875000 & -1.433254000 & 2.000582000 \\
\hline
\end{tabular}




\begin{tabular}{|c|c|c|c|c|c|c|c|}
\hline H & -0.979451000 & -1.467263000 & 1.440750000 & $\mathrm{H}$ & -2.148709000 & -1.669499000 & 1.116188000 \\
\hline $\mathrm{H}$ & -2.245155000 & -2.819450000 & -0.209485000 & $\mathrm{H}$ & -3.383965000 & -2.651479000 & -0.796984000 \\
\hline $\mathrm{H}$ & -2.601287000 & -3.426210000 & 1.406504000 & $\mathrm{H}$ & -3.792663000 & -3.555198000 & 0.660049000 \\
\hline $\mathrm{H}$ & -4.776793000 & -2.471286000 & 1.459022000 & $\mathrm{H}$ & -5.958694000 & -2.588921000 & 0.840832000 \\
\hline $\mathrm{H}$ & -4.658067000 & -3.053547000 & -0.165652000 & H & -5.798988000 & -2.850224000 & -0.861874000 \\
\hline $\mathrm{H}$ & -6.609075000 & -1.815077000 & 0.051682000 & $\mathrm{H}$ & -7.739651000 & -1.640364000 & -0.467041000 \\
\hline $\mathrm{H}$ & -6.632593000 & -0.050619000 & 0.185698000 & $\mathrm{H}$ & -7.745215000 & 0.065662000 & 0.003533000 \\
\hline $\mathrm{H}$ & -6.256493000 & -0.815443000 & -1.354843000 & $\mathrm{H}$ & -7.329976000 & -0.396161000 & -1.643947000 \\
\hline $\mathrm{H}$ & -2.878923000 & 3.163024000 & -0.221308000 & $\mathrm{H}$ & -3.942335000 & 3.227330000 & 0.343476000 \\
\hline $\mathrm{H}$ & -2.633352000 & 2.230472000 & 1.257499000 & H & -3.761530000 & 2.020828000 & 1.619409000 \\
\hline $\mathrm{H}$ & -4.091978000 & 3.270068000 & 1.033990000 & $\mathrm{H}$ & -5.199636000 & 3.110793000 & 1.553293000 \\
\hline $\mathrm{H}$ & -5.213626000 & 2.927791000 & -1.193265000 & $\mathrm{H}$ & -6.243984000 & 3.225916000 & -0.736328000 \\
\hline $\mathrm{H}$ & -6.227052000 & 2.032973000 & -0.085818000 & $\mathrm{H}$ & -7.306976000 & 2.152362000 & 0.142942000 \\
\hline $\mathrm{H}$ & -5.706753000 & 1.269874000 & -1.600481000 & $\mathrm{H}$ & -6.739053000 & 1.685445000 & -1.471532000 \\
\hline $\mathrm{H}$ & -1.661367000 & 1.182341000 & -1.086122000 & $\mathrm{H}$ & -2.727432000 & 1.431899000 & -0.865175000 \\
\hline $\mathrm{Au}$ & 0.718685000 & 0.226589000 & -0.225025000 & $\mathrm{Au}$ & -0.389021000 & 0.281428000 & -0.142508000 \\
\hline C & -1.315716000 & 0.457045000 & -0.343278000 & C & -2.412075000 & 0.571452000 & -0.267620000 \\
\hline$P$ & 3.107653000 & 0.029742000 & -0.141071000 & $\mathrm{P}$ & 1.997195000 & 0.002245000 & -0.024531000 \\
\hline C & 3.844208000 & -0.847966000 & -1.597343000 & C & 2.884002000 & 1.599159000 & 0.145441000 \\
\hline $\mathrm{H}$ & 4.934660000 & -0.851363000 & -1.487485000 & $\mathrm{H}$ & 1.526194000 & 2.607129000 & -1.201879000 \\
\hline H & 3.614941000 & -0.225576000 & -2.469663000 & C & 2.410538000 & 2.706134000 & -0.577972000 \\
\hline C & 3.694274000 & -0.876793000 & 1.367826000 & C & 3.069863000 & 3.931886000 & -0.498320000 \\
\hline $\mathrm{H}$ & 3.188222000 & -1.848435000 & 1.353803000 & C & 4.200026000 & 4.067179000 & 0.311689000 \\
\hline $\mathrm{H}$ & 3.275175000 & -0.336251000 & 2.223766000 & $\mathrm{H}$ & 2.698429000 & 4.781895000 & -1.062724000 \\
\hline C & 3.979960000 & 1.665555000 & -0.130931000 & $\mathrm{H}$ & 4.709250000 & 5.023872000 & 0.378336000 \\
\hline H & 5.060833000 & 1.485691000 & -0.153846000 & C & 4.670857000 & 2.973365000 & 1.039591000 \\
\hline $\mathrm{H}$ & 3.718396000 & 2.151852000 & -1.077403000 & $\mathrm{H}$ & 5.546400000 & 3.076457000 & 1.673469000 \\
\hline C & 5.210069000 & -1.053080000 & 1.522176000 & C & 4.017841000 & 1.741898000 & 0.958781000 \\
\hline H & 5.641404000 & -1.628912000 & 0.698242000 & H & 4.387750000 & 0.897979000 & 1.532029000 \\
\hline $\mathrm{H}$ & 5.731199000 & -0.092595000 & 1.573269000 & C & 1.826193000 & -0.867824000 & 2.621260000 \\
\hline $\mathrm{H}$ & 5.429755000 & -1.593228000 & 2.447996000 & $\mathrm{H}$ & 0.991962000 & -0.174008000 & 2.685024000 \\
\hline C & 3.598661000 & 2.563801000 & 1.052714000 & C & 2.204409000 & -1.604024000 & 3.743176000 \\
\hline $\mathrm{H}$ & 3.893352000 & 2.122838000 & 2.009858000 & H & 1.666153000 & -1.478155000 & 4.677823000 \\
\hline $\mathrm{H}$ & 4.101198000 & 3.531715000 & 0.969053000 & C & 3.268246000 & -2.505975000 & 3.662050000 \\
\hline $\mathrm{H}$ & 2.519612000 & 2.745028000 & 1.082361000 & $\mathrm{H}$ & 3.558548000 & -3.083666000 & 4.534427000 \\
\hline C & 3.309592000 & -2.270926000 & -1.804522000 & C & 3.954562000 & -2.667482000 & 2.457513000 \\
\hline H & 3.555236000 & -2.925369000 & -0.962555000 & $\mathrm{H}$ & 4.779190000 & -3.370654000 & 2.390084000 \\
\hline $\mathrm{H}$ & 2.222207000 & -2.272529000 & -1.928821000 & C & 3.584080000 & -1.927776000 & 1.332721000 \\
\hline \multirow[t]{8}{*}{$\mathrm{H}$} & 3.751570000 & -2.712338000 & -2.702446000 & $\mathrm{H}$ & 4.121155000 & -2.062310000 & 0.399436000 \\
\hline & & & & C & 2.518166000 & -1.019237000 & 1.408137000 \\
\hline & & & & C & 2.699382000 & -0.807644000 & -1.512828000 \\
\hline & & & & C & 3.968607000 & -0.476207000 & -2.010888000 \\
\hline & & & & $\mathrm{H}$ & 4.558345000 & 0.296784000 & -1.528716000 \\
\hline & & & & C & 4.473569000 & -1.133328000 & -3.134083000 \\
\hline & & & & H & 5.455253000 & -0.868876000 & -3.515293000 \\
\hline & & & & $\mathrm{C}$ & 3.719058000 & -2.122667000 & -3.767165000 \\
\hline
\end{tabular}




\begin{tabular}{|c|c|c|c|c|c|c|c|}
\hline & & & & $\mathrm{H}$ & 4.113715000 & -2.629929000 & -4.642340000 \\
\hline & & & & C & 2.453293000 & -2.454501000 & -3.278670000 \\
\hline & & & & $\mathrm{H}$ & 1.861185000 & -3.218778000 & -3.772942000 \\
\hline & & & & $\mathrm{H}$ & 0.951645000 & -2.048813000 & -1.790162000 \\
\hline & & & & C & 1.942146000 & -1.797589000 & -2.160000000 \\
\hline & & Int2 $\left(t \mathrm{Bu}_{3} \mathbf{P A u ^ { + }}\right)$ & & & & Int2 $\left.(\operatorname{IPrAu})^{+}\right)$ & \\
\hline C & 4.796478000 & -1.519108000 & 0.145263000 & C & -4.912771000 & -2.340977000 & -0.602532000 \\
\hline c & 5.322017000 & 0.862548000 & -0.227661000 & c & -5.834875000 & -0.328298000 & 0.478087000 \\
\hline C & 4.386828000 & -0.166496000 & 0.005908000 & C & -4.744800000 & -1.036323000 & -0.064990000 \\
\hline c & 2.961154000 & 0.201131000 & 0.274529000 & c & -3.434310000 & -0.338063000 & -0.230990000 \\
\hline c & 2.762398000 & 1.515388000 & 0.992756000 & c & -3.523338000 & 1.146788000 & -0.497253000 \\
\hline C & 3.440769000 & 2.615889000 & 0.168190000 & C & -4.333687000 & 1.788577000 & 0.635330000 \\
\hline c & 4.924431000 & 2.297049000 & -0.028749000 & c & -5.720750000 & 1.148738000 & 0.725730000 \\
\hline C & 6.775912000 & 0.710861000 & -0.569969000 & C & -7.212288000 & -0.854627000 & 0.764434000 \\
\hline C & 4.034906000 & -2.466304000 & 1.013450000 & C & -4.082508000 & -2.809720000 & -1.752276000 \\
\hline C & 5.988230000 & -2.141131000 & -0.509478000 & C & -5.892620000 & -3.361169000 & -0.118528000 \\
\hline H & 3.200149000 & 1.486412000 & 2.002004000 & H & -4.015584000 & 1.348085000 & -1.461055000 \\
\hline H & 1.694710000 & 1.716237000 & 1.102286000 & H & -2.519921000 & 1.573739000 & -0.545524000 \\
\hline H & 2.946478000 & 2.682616000 & -0.807176000 & H & -3.798121000 & 1.644288000 & 1.580089000 \\
\hline H & 3.347139000 & 3.596164000 & 0.646282000 & H & -4.447625000 & 2.867700000 & 0.490438000 \\
\hline $\mathrm{H}$ & 5.508195000 & 2.622191000 & 0.851567000 & $\mathrm{H}$ & -6.403111000 & 1.605437000 & -0.013786000 \\
\hline $\mathrm{H}$ & 5.362679000 & 2.878198000 & -0.853457000 & $\mathrm{H}$ & -6.209258000 & 1.359520000 & 1.688688000 \\
\hline H & 7.295142000 & 1.663127000 & -0.446197000 & H & -7.913350000 & -0.026686000 & 0.889206000 \\
\hline H & 7.293839000 & -0.042134000 & 0.026747000 & H & -7.603499000 & -1.523334000 & -0.003212000 \\
\hline $\mathrm{H}$ & 6.885428000 & 0.418836000 & -1.622776000 & $\mathrm{H}$ & -7.207492000 & -1.413103000 & 1.710220000 \\
\hline $\mathrm{H}$ & 3.489893000 & -3.191669000 & 0.394049000 & H & -3.344263000 & -3.548667000 & -1.412747000 \\
\hline $\mathrm{H}$ & 3.309395000 & -1.969346000 & 1.655288000 & $\mathrm{H}$ & -3.537831000 & -2.002412000 & -2.239589000 \\
\hline $\mathrm{H}$ & 4.748216000 & -3.059001000 & 1.601222000 & H & -4.728485000 & -3.341513000 & -2.463313000 \\
\hline $\mathrm{H}$ & 5.782306000 & -3.198898000 & -0.700298000 & $\mathrm{H}$ & -5.447917000 & -4.356818000 & -0.219859000 \\
\hline $\mathrm{H}$ & 6.850452000 & -2.127734000 & 0.175221000 & H & -6.783931000 & -3.372883000 & -0.764845000 \\
\hline $\mathrm{H}$ & 6.284901000 & -1.663733000 & -1.441255000 & $\mathrm{H}$ & -6.213824000 & -3.221326000 & 0.911437000 \\
\hline $\mathrm{H}$ & 2.278842000 & -1.405702000 & -0.836956000 & $\mathrm{H}$ & -2.405904000 & -2.049169000 & 0.297928000 \\
\hline $\mathrm{Au}$ & -0.071819000 & -0.254104000 & -0.127585000 & $\mathrm{Au}$ & -0.346701000 & -0.323537000 & -0.006085000 \\
\hline C & 1.958549000 & -0.539563000 & -0.249122000 & C & -2.277689000 & -0.998870000 & 0.013414000 \\
\hline$P$ & -2.477793000 & 0.027088000 & -0.011339000 & C & 1.631274000 & 0.303193000 & 0.021021000 \\
\hline C & -2.949532000 & 1.715118000 & -0.837089000 & N & 2.107322000 & 1.577705000 & -0.046291000 \\
\hline C & -3.015615000 & 0.019624000 & 1.849898000 & C & 3.495501000 & 1.595479000 & 0.020666000 \\
\hline C & -3.320386000 & -1.438745000 & -0.956600000 & $\mathrm{H}$ & 4.056970000 & 2.515030000 & -0.017045000 \\
\hline C & -4.438733000 & 0.557757000 & 2.104084000 & C & 3.896474000 & 0.303716000 & 0.132484000 \\
\hline H & -4.536885000 & 1.617853000 & 1.861813000 & H & 4.879144000 & -0.132720000 & 0.210827000 \\
\hline $\mathrm{H}$ & -5.201889000 & 0.008508000 & 1.549735000 & C & 0.948442000 & 3.222206000 & -1.454973000 \\
\hline $\mathrm{H}$ & -4.669646000 & 0.450649000 & 3.170560000 & C & 1.290819000 & 2.766719000 & -0.166043000 \\
\hline C & -4.822421000 & -1.616353000 & -0.651615000 & C & 0.891540000 & 3.430552000 & 1.010916000 \\
\hline H & -5.406186000 & -0.718985000 & -0.865957000 & C & 1.269584000 & 2.945772000 & 2.407437000 \\
\hline $\mathrm{H}$ & -5.215690000 & -2.420321000 & -1.284949000 & C & 0.021094000 & 2.550615000 & 3.219879000 \\
\hline $\mathrm{H}$ & -5.011220000 & -1.906920000 & 0.383850000 & $\mathrm{H}$ & -0.552039000 & 1.773114000 & 2.704742000 \\
\hline
\end{tabular}




\begin{tabular}{|c|c|c|c|c|c|c|c|}
\hline c & -2.532344000 & 2.875012000 & 0.093951000 & $\mathrm{H}$ & 0.312891000 & 2.166414000 & 4.202983000 \\
\hline $\mathrm{H}$ & -3.151014000 & 2.950556000 & 0.989203000 & $\mathrm{H}$ & -0.638545000 & 3.409776000 & 3.382242000 \\
\hline $\mathrm{H}$ & -1.482879000 & 2.800184000 & 0.394080000 & $\mathrm{H}$ & 1.883173000 & 2.046479000 & 2.303119000 \\
\hline $\mathrm{H}$ & -2.649984000 & 3.814726000 & -0.457493000 & $\mathrm{H}$ & 2.420064000 & 3.603767000 & 4.136498000 \\
\hline c & -2.112828000 & 1.885051000 & -2.128491000 & C & 2.116366000 & 3.991768000 & 3.158838000 \\
\hline $\mathrm{H}$ & -1.039997000 & 1.834366000 & -1.920496000 & $\mathrm{H}$ & 3.021752000 & 4.250508000 & 2.600734000 \\
\hline H & -2.343021000 & 1.150491000 & -2.897938000 & $\mathrm{H}$ & 1.556175000 & 4.916923000 & 3.329120000 \\
\hline $\mathrm{H}$ & -2.326674000 & 2.875149000 & -2.547921000 & $\mathrm{H}$ & -0.208114000 & 5.124503000 & 1.750730000 \\
\hline C & -4.446497000 & 1.866402000 & -1.176805000 & C & 0.113866000 & 4.586069000 & 0.864679000 \\
\hline $\mathrm{H}$ & -4.613003000 & 2.869443000 & -1.587075000 & C & -0.247212000 & 5.057095000 & -0.394569000 \\
\hline $\mathrm{H}$ & -4.778984000 & 1.153820000 & -1.933900000 & $\mathrm{H}$ & -0.847071000 & 5.958107000 & -0.484838000 \\
\hline $\mathrm{H}$ & -5.092424000 & 1.762490000 & -0.302727000 & C & 0.166640000 & 4.381184000 & -1.539302000 \\
\hline C & -1.999064000 & 0.855223000 & 2.665853000 & $\mathrm{H}$ & -0.115760000 & 4.762112000 & -2.515894000 \\
\hline H & -2.278620000 & 0.796098000 & 3.724317000 & $\mathrm{H}$ & 3.162662000 & 3.775962000 & -3.042276000 \\
\hline $\mathrm{H}$ & -0.985068000 & 0.456681000 & 2.565880000 & C & 2.287126000 & 3.425974000 & -3.598101000 \\
\hline $\mathrm{H}$ & -1.979435000 & 1.909143000 & 2.394118000 & $\mathrm{H}$ & 1.741894000 & 4.308161000 & -3.948978000 \\
\hline c & -2.920588000 & -1.417547000 & 2.408295000 & $\mathrm{H}$ & 2.640416000 & 2.882663000 & -4.480504000 \\
\hline $\mathrm{H}$ & -3.078076000 & -1.372104000 & 3.491824000 & C & 0.191658000 & 1.986105000 & -3.534408000 \\
\hline H & -3.680034000 & -2.087218000 & 2.003088000 & $\mathrm{H}$ & -0.411939000 & 1.299352000 & -2.932380000 \\
\hline $\mathrm{H}$ & -1.932943000 & -1.857060000 & 2.239328000 & $\mathrm{H}$ & -0.456068000 & 2.802381000 & -3.871546000 \\
\hline C & -3.143811000 & -1.240623000 & -2.478556000 & $\mathrm{H}$ & 0.536737000 & 1.448853000 & -4.424084000 \\
\hline $\mathrm{H}$ & -2.097587000 & -1.075254000 & -2.752118000 & C & 1.395666000 & 2.515311000 & -2.731110000 \\
\hline $\mathrm{H}$ & -3.472988000 & -2.157226000 & -2.980936000 & $\mathrm{H}$ & 1.997471000 & 1.647618000 & -2.446042000 \\
\hline $\mathrm{H}$ & -3.747266000 & -0.424345000 & -2.877969000 & $\mathrm{~N}$ & 2.744561000 & -0.473757000 & 0.131275000 \\
\hline C & -2.568243000 & -2.747488000 & -0.611177000 & C & 2.748830000 & -1.918736000 & 0.225682000 \\
\hline H & -2.632748000 & -3.025916000 & 0.438936000 & C & 2.685454000 & -2.511677000 & 1.502283000 \\
\hline $\mathrm{H}$ & -3.012485000 & -3.562712000 & -1.194254000 & C & 2.842728000 & -2.669331000 & -0.963056000 \\
\hline \multirow[t]{19}{*}{$\mathrm{H}$} & -1.510075000 & -2.681920000 & -0.881413000 & C & 2.916570000 & -2.029557000 & -2.346294000 \\
\hline & & & & $\mathrm{H}$ & 2.931670000 & -0.943105000 & -2.220260000 \\
\hline & & & & $\mathrm{H}$ & 0.755337000 & -2.045767000 & -2.679974000 \\
\hline & & & & C & 1.670002000 & -2.371180000 & -3.185855000 \\
\hline & & & & $\mathrm{H}$ & 1.593629000 & -3.448675000 & -3.366850000 \\
\hline & & & & $\mathrm{H}$ & 1.719293000 & -1.873470000 & -4.160246000 \\
\hline & & & & $\mathrm{H}$ & 4.267844000 & -1.901623000 & -4.049108000 \\
\hline & & & & C & 4.210854000 & -2.419664000 & -3.086316000 \\
\hline & & & & $\mathrm{H}$ & 5.100162000 & -2.155128000 & -2.505646000 \\
\hline & & & & $\mathrm{H}$ & 4.253168000 & -3.494999000 & -3.287295000 \\
\hline & & & & $\mathrm{H}$ & 2.934845000 & -4.674865000 & -1.736406000 \\
\hline & & & & C & 2.862238000 & -4.064246000 & -0.841705000 \\
\hline & & & & $\mathrm{H}$ & 2.817390000 & -5.763835000 & 0.475663000 \\
\hline & & & & C & 2.795954000 & -4.680237000 & 0.404951000 \\
\hline & & & & $\mathrm{H}$ & 2.664177000 & -4.402432000 & 2.528452000 \\
\hline & & & & C & 2.709728000 & -3.910622000 & 1.561657000 \\
\hline & & & & $\mathrm{H}$ & 2.579778000 & -0.639986000 & 2.527185000 \\
\hline & & & & C & 2.599506000 & -1.701049000 & 2.791920000 \\
\hline & & & & C & 3.836797000 & -1.928447000 & 3.682736000 \\
\hline
\end{tabular}




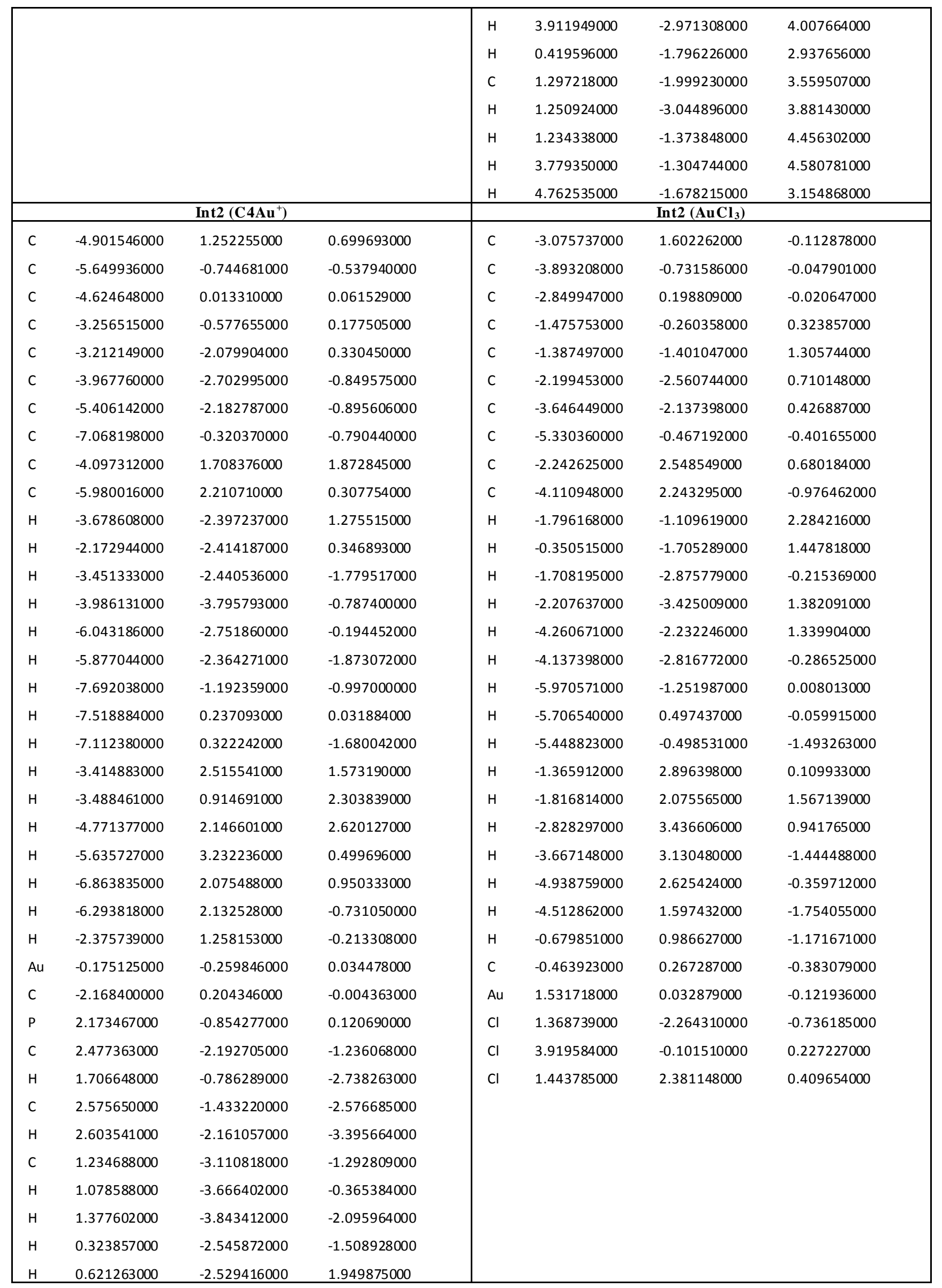




\begin{tabular}{|c|c|c|c|c|c|c|c|}
\hline c & 1.674490000 & -2.706275000 & 2.189720000 & & & & \\
\hline $\mathrm{H}$ & 2.014291000 & -3.588208000 & 1.640009000 & & & & \\
\hline $\mathrm{H}$ & 1.739334000 & -2.945397000 & 3.257443000 & & & & \\
\hline $\mathrm{H}$ & 3.671094000 & -3.680638000 & -0.138451000 & & & & \\
\hline c & 3.719629000 & -3.084756000 & -1.053040000 & & & & \\
\hline $\mathrm{H}$ & 3.767262000 & -3.787827000 & -1.892866000 & & & & \\
\hline $\mathrm{H}$ & 4.656985000 & -2.526193000 & -1.057305000 & & & & \\
\hline $\mathrm{H}$ & 4.425056000 & -2.583034000 & 1.572153000 & & & & \\
\hline C & 4.019303000 & -1.794636000 & 2.209475000 & & & & \\
\hline $\mathrm{H}$ & 4.085039000 & -2.149086000 & 3.244828000 & & & & \\
\hline $\mathrm{H}$ & 4.660534000 & -0.914674000 & 2.127562000 & & & & \\
\hline c & 2.546106000 & -1.466647000 & 1.901646000 & & & & \\
\hline C & 2.104023000 & -0.317346000 & 2.835170000 & & & & \\
\hline $\mathrm{H}$ & 2.268756000 & -0.620908000 & 3.875511000 & & & & \\
\hline $\mathrm{H}$ & 2.685853000 & 0.592895000 & 2.661261000 & & & & \\
\hline $\mathrm{H}$ & 1.043208000 & -0.078232000 & 2.714752000 & & & & \\
\hline C & 3.398409000 & 0.506268000 & -0.198322000 & & & & \\
\hline C & 4.766764000 & 0.172476000 & -0.273495000 & & & & \\
\hline H & 5.076902000 & -0.853042000 & -0.129594000 & & & & \\
\hline C & 5.752748000 & 1.122079000 & -0.524350000 & & & & \\
\hline $\mathrm{H}$ & 6.793901000 & 0.818694000 & -0.575162000 & & & & \\
\hline $\mathrm{H}$ & 6.142555000 & 3.212052000 & -0.901795000 & & & & \\
\hline C & 5.389708000 & 2.454676000 & -0.705931000 & & & & \\
\hline C & 4.046557000 & 2.808997000 & -0.633814000 & & & & \\
\hline $\mathrm{H}$ & 3.756496000 & 3.845756000 & -0.774061000 & & & & \\
\hline C & 3.034443000 & 1.863379000 & -0.385006000 & & & & \\
\hline C & 1.646300000 & 2.432771000 & -0.350700000 & & & & \\
\hline C & 0.933754000 & 2.627122000 & -1.544737000 & & & & \\
\hline $\mathrm{H}$ & 1.345702000 & 2.254308000 & -2.477836000 & & & & \\
\hline C & -0.278219000 & 3.319562000 & -1.546592000 & & & & \\
\hline $\mathrm{H}$ & -0.805975000 & 3.475829000 & -2.482998000 & & & & \\
\hline $\mathrm{H}$ & -1.717729000 & 4.406181000 & -0.362882000 & & & & \\
\hline C & -0.793443000 & 3.834542000 & -0.354734000 & & & & \\
\hline H & -0.477103000 & 4.056605000 & 1.769531000 & & & & \\
\hline C & -0.095967000 & 3.642460000 & 0.840132000 & & & & \\
\hline C & 1.116588000 & 2.949656000 & 0.841166000 & & & & \\
\hline $\mathrm{H}$ & 1.670448000 & 2.832076000 & 1.767691000 & & & & \\
\hline $\mathrm{H}$ & 3.477514000 & -0.820147000 & -2.640477000 & & & & \\
\hline \multicolumn{4}{|c|}{$\mathbf{T S b}\left(\mathrm{H}_{3} \mathrm{PAu}^{+}\right)$} & \multicolumn{4}{|c|}{$\mathbf{T S b}\left(\mathrm{Me}_{3} \mathbf{P A u}^{+}\right)$} \\
\hline C & -2.851571000 & -1.668758000 & 0.046752000 & C & -3.470373000 & -1.646997000 & 0.086027000 \\
\hline C & -3.722517000 & 0.679396000 & -0.113584000 & C & -4.305975000 & 0.712493000 & -0.058012000 \\
\hline C & -2.669391000 & -0.223242000 & -0.082267000 & C & -3.268629000 & -0.206347000 & -0.054878000 \\
\hline C & -1.284082000 & 0.308784000 & -0.066619000 & C & -1.870870000 & 0.302017000 & -0.090564000 \\
\hline C & -1.138405000 & 1.640476000 & 0.628169000 & C & -1.684674000 & 1.639481000 & 0.585682000 \\
\hline C & -2.072383000 & 2.644821000 & -0.066033000 & C & -2.626801000 & 2.651712000 & -0.084246000 \\
\hline C & -3.520839000 & 2.162903000 & 0.022847000 & C & -4.078169000 & 2.194195000 & 0.062786000 \\
\hline
\end{tabular}




\begin{tabular}{|c|c|c|c|c|c|c|c|}
\hline c & -5.140835000 & 0.275681000 & -0.370429000 & C & -5.738839000 & 0.329612000 & -0.265473000 \\
\hline c & -1.901889000 & -2.527967000 & -0.509499000 & C & -2.554312000 & -2.519603000 & -0.504967000 \\
\hline c & -3.807494000 & -2.213052000 & 1.079433000 & c & -4.390747000 & -2.173226000 & 1.159771000 \\
\hline $\mathrm{H}$ & -0.102274000 & 1.975926000 & 0.578066000 & $\mathrm{H}$ & -0.645600000 & 1.957034000 & 0.496804000 \\
\hline $\mathrm{H}$ & -1.417733000 & 1.553916000 & 1.688293000 & $\mathrm{H}$ & -1.928385000 & 1.567063000 & 1.655881000 \\
\hline $\mathrm{H}$ & -1.973460000 & 3.631438000 & 0.395183000 & $\mathrm{H}$ & -2.495839000 & 3.641923000 & 0.361375000 \\
\hline $\mathrm{H}$ & -1.778362000 & 2.750378000 & -1.116684000 & H & -2.369917000 & 2.740777000 & -1.146239000 \\
\hline $\mathrm{H}$ & -4.170193000 & 2.691761000 & -0.684164000 & $\mathrm{H}$ & -4.744792000 & 2.728688000 & -0.623739000 \\
\hline $\mathrm{H}$ & -3.931481000 & 2.403954000 & 1.019418000 & H & -4.446627000 & 2.448722000 & 1.072282000 \\
\hline $\mathrm{H}$ & -5.440249000 & 0.674205000 & -1.349294000 & $\mathrm{H}$ & -6.063804000 & 0.719760000 & -1.239513000 \\
\hline $\mathrm{H}$ & -5.308038000 & -0.798687000 & -0.385284000 & $\mathrm{H}$ & -5.922804000 & -0.742265000 & -0.260462000 \\
\hline $\mathrm{H}$ & -5.811258000 & 0.744440000 & 0.359751000 & $\mathrm{H}$ & -6.377988000 & 0.817578000 & 0.479979000 \\
\hline $\mathrm{H}$ & -0.793579000 & -1.702262000 & -0.203651000 & H & -1.426236000 & -1.713656000 & -0.239487000 \\
\hline H & -1.644137000 & -2.436410000 & -1.561408000 & H & -2.337547000 & -2.432569000 & -1.566494000 \\
\hline $\mathrm{H}$ & -1.880752000 & -3.556486000 & -0.152387000 & H & -2.533517000 & -3.548299000 & -0.147822000 \\
\hline $\mathrm{H}$ & -3.250264000 & -2.868750000 & 1.759989000 & $\mathrm{H}$ & -3.817624000 & -2.836231000 & 1.819779000 \\
\hline $\mathrm{H}$ & -4.576548000 & -2.843263000 & 0.617166000 & $\mathrm{H}$ & -5.190013000 & -2.790724000 & 0.732751000 \\
\hline $\mathrm{H}$ & -4.289804000 & -1.441387000 & 1.679954000 & $\mathrm{H}$ & -4.834292000 & -1.390609000 & 1.776023000 \\
\hline C & -0.203077000 & -0.430565000 & -0.543057000 & c & -0.812034000 & -0.450155000 & -0.590079000 \\
\hline $\mathrm{Au}$ & 1.780347000 & -0.093932000 & -0.104108000 & $\mathrm{Au}$ & 1.202679000 & -0.144821000 & -0.197537000 \\
\hline$P$ & 4.111133000 & 0.187711000 & 0.366501000 & $P$ & 3.542298000 & 0.096752000 & 0.213821000 \\
\hline $\mathrm{H}$ & -0.312306000 & -0.853028000 & -1.541718000 & $\mathrm{H}$ & -0.950270000 & -0.887396000 & -1.579056000 \\
\hline $\mathrm{H}$ & 4.590094000 & -0.255770000 & 1.614514000 & C & 4.574398000 & -0.069908000 & -1.299109000 \\
\hline $\mathrm{H}$ & 4.628940000 & 1.497241000 & 0.350333000 & $\mathrm{H}$ & 5.636296000 & 0.045499000 & -1.061980000 \\
\hline \multirow[t]{11}{*}{$\mathrm{H}$} & 5.021870000 & -0.453105000 & -0.495335000 & $\mathrm{H}$ & 4.410406000 & -1.053365000 & -1.746643000 \\
\hline & & & & $\mathrm{H}$ & 4.282711000 & 0.691581000 & -2.026639000 \\
\hline & & & & C & 4.232162000 & -1.142788000 & 1.384570000 \\
\hline & & & & $\mathrm{H}$ & 3.725142000 & -1.058140000 & 2.348935000 \\
\hline & & & & $\mathrm{H}$ & 4.062799000 & -2.149253000 & 0.993898000 \\
\hline & & & & $\mathrm{H}$ & 5.305947000 & -0.987528000 & 1.526596000 \\
\hline & & & & C & 4.023817000 & 1.722238000 & 0.927514000 \\
\hline & & & & H & 3.506576000 & 1.872269000 & 1.878436000 \\
\hline & & & & H & 5.104146000 & 1.768403000 & 1.094498000 \\
\hline & & & & $\mathrm{H}$ & 3.731428000 & 2.523946000 & 0.244788000 \\
\hline & \multicolumn{3}{|c|}{ 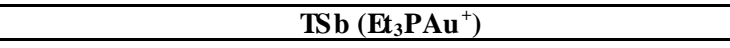 } & \multicolumn{4}{|c|}{$\mathbf{T S b}\left(\mathrm{Ph}_{3} \mathrm{PAu}^{+}\right)$} \\
\hline C & -3.963676000 & -1.654734000 & -0.131526000 & C & -5.062936000 & -1.675176000 & 0.012940000 \\
\hline C & -4.788929000 & 0.711299000 & -0.036466000 & C & -5.866077000 & 0.697399000 & -0.068041000 \\
\hline C & -3.755805000 & -0.208634000 & -0.113339000 & C & -4.842853000 & -0.235689000 & -0.104451000 \\
\hline C & -2.355908000 & 0.294696000 & -0.072051000 & c & -3.437559000 & 0.253469000 & -0.163174000 \\
\hline C & -2.177232000 & 1.547859000 & 0.751736000 & C & -3.217600000 & 1.578621000 & 0.528362000 \\
\hline C & -3.103306000 & 2.633453000 & 0.181948000 & C & -4.162460000 & 2.613927000 & -0.100233000 \\
\hline C & -4.558628000 & 2.169659000 & 0.251604000 & C & -5.615560000 & 2.173514000 & 0.076577000 \\
\hline C & -6.218796000 & 0.362753000 & -0.313993000 & C & -7.308642000 & 0.338485000 & -0.251916000 \\
\hline C & -3.037739000 & -2.463807000 & -0.793747000 & C & -4.171675000 & -2.547503000 & -0.615894000 \\
\hline C & -4.908561000 & -2.288318000 & 0.859896000 & C & -5.964840000 & -2.209746000 & 1.098178000 \\
\hline $\mathrm{H}$ & -1.135272000 & 1.866461000 & 0.717570000 & $\mathrm{H}$ & -2.177070000 & 1.884258000 & 0.418136000 \\
\hline
\end{tabular}




\begin{tabular}{|c|c|c|c|c|c|c|c|}
\hline H & -2.441134000 & 1.359005000 & 1.802791000 & H & -3.434775000 & 1.491640000 & 1.603340000 \\
\hline $\mathrm{H}$ & -2.977592000 & 3.567468000 & 0.736998000 & $\mathrm{H}$ & -4.006253000 & 3.595002000 & 0.357360000 \\
\hline $\mathrm{H}$ & -2.827088000 & 2.838183000 & -0.859042000 & $\mathrm{H}$ & -3.931522000 & 2.716618000 & -1.167001000 \\
\hline $\mathrm{H}$ & -5.210708000 & 2.780210000 & -0.383506000 & $\mathrm{H}$ & -6.292415000 & 2.730358000 & -0.581626000 \\
\hline $\mathrm{H}$ & -4.944424000 & 2.313510000 & 1.276340000 & $\mathrm{H}$ & -5.953448000 & 2.412980000 & 1.100375000 \\
\hline $\mathrm{H}$ & -6.524262000 & 0.865008000 & -1.241781000 & $\mathrm{H}$ & -7.649989000 & 0.753688000 & -1.209810000 \\
\hline $\mathrm{H}$ & -6.405985000 & -0.701561000 & -0.436718000 & H & -7.507192000 & -0.730768000 & -0.265374000 \\
\hline $\mathrm{H}$ & -6.870485000 & 0.763903000 & 0.471411000 & $\mathrm{H}$ & -7.924264000 & 0.818523000 & 0.518141000 \\
\hline $\mathrm{H}$ & -1.912368000 & -1.690553000 & -0.429314000 & H & -3.028791000 & -1.765721000 & -0.359356000 \\
\hline $\mathrm{H}$ & -2.801888000 & -2.268558000 & -1.836560000 & $\mathrm{H}$ & -3.979470000 & -2.442602000 & -1.680600000 \\
\hline $\mathrm{H}$ & -3.025291000 & -3.524200000 & -0.545154000 & $\mathrm{H}$ & -4.156229000 & -3.583340000 & -0.279440000 \\
\hline $\mathrm{H}$ & -4.351364000 & -3.017944000 & 1.460707000 & $\mathrm{H}$ & -5.385906000 & -2.892540000 & 1.732505000 \\
\hline $\mathrm{H}$ & -5.697599000 & -2.855809000 & 0.352158000 & H & -6.782754000 & -2.808025000 & 0.679083000 \\
\hline $\mathrm{H}$ & -5.367010000 & -1.573589000 & 1.544078000 & H & -6.383011000 & -1.432636000 & 1.738768000 \\
\hline C & -1.291851000 & -0.399986000 & -0.638195000 & C & -2.399489000 & -0.503709000 & -0.695158000 \\
\hline $\mathrm{Au}$ & 0.723149000 & -0.137856000 & -0.197161000 & $\mathrm{Au}$ & -0.373802000 & -0.226049000 & -0.330048000 \\
\hline$P$ & 3.058153000 & 0.071459000 & 0.265462000 & $\mathrm{H}$ & -2.555020000 & -0.924125000 & -1.688776000 \\
\hline $\mathrm{H}$ & -1.420279000 & -0.728320000 & -1.669855000 & $P$ & 1.981849000 & 0.004031000 & 0.039875000 \\
\hline C & 4.080172000 & -0.162216000 & -1.263546000 & C & 2.576317000 & 1.699198000 & -0.325578000 \\
\hline $\mathrm{H}$ & 3.799342000 & -1.140706000 & -1.668466000 & $\mathrm{H}$ & 1.249745000 & 1.906870000 & -2.020955000 \\
\hline $\mathrm{H}$ & 3.712410000 & 0.574593000 & -1.986155000 & C & 2.025131000 & 2.379751000 & -1.424038000 \\
\hline C & 3.686067000 & -1.144065000 & 1.514147000 & C & 2.468960000 & 3.659990000 & -1.751835000 \\
\hline $\mathrm{H}$ & 3.162306000 & -0.913044000 & 2.448538000 & C & 3.457378000 & 4.276547000 & -0.980564000 \\
\hline $\mathrm{H}$ & 4.749216000 & -0.942565000 & 1.687725000 & $\mathrm{H}$ & 2.040340000 & 4.177709000 & -2.604595000 \\
\hline C & 3.536244000 & 1.716942000 & 0.970381000 & $\mathrm{H}$ & 3.798278000 & 5.276116000 & -1.232736000 \\
\hline H & 3.013495000 & 1.796636000 & 1.930176000 & C & 4.003206000 & 3.608984000 & 0.116964000 \\
\hline $\mathrm{H}$ & 4.608989000 & 1.699381000 & 1.194023000 & $\mathrm{H}$ & 4.768738000 & 4.087208000 & 0.720408000 \\
\hline C & 3.185883000 & 2.908674000 & 0.070182000 & C & 3.567139000 & 2.324148000 & 0.446193000 \\
\hline $\mathrm{H}$ & 3.734341000 & 2.880721000 & -0.876152000 & $\mathrm{H}$ & 3.994037000 & 1.814231000 & 1.303677000 \\
\hline $\mathrm{H}$ & 2.116425000 & 2.932649000 & -0.161397000 & C & 1.615425000 & 0.018165000 & 2.808172000 \\
\hline H & 3.443648000 & 3.846497000 & 0.570479000 & H & 0.664000000 & 0.490325000 & 2.578403000 \\
\hline C & 5.601307000 & -0.054079000 & -1.097212000 & C & 1.972424000 & -0.214857000 & 4.135539000 \\
\hline $\mathrm{H}$ & 5.993976000 & -0.811539000 & -0.412762000 & H & 1.300102000 & 0.080559000 & 4.935330000 \\
\hline $\mathrm{H}$ & 6.091149000 & -0.202411000 & -2.064111000 & C & 3.187891000 & -0.835163000 & 4.434179000 \\
\hline $\mathrm{H}$ & 5.904266000 & 0.929020000 & -0.725328000 & $\mathrm{H}$ & 3.462528000 & -1.022531000 & 5.467798000 \\
\hline C & 3.463294000 & -2.611414000 & 1.126043000 & C & 4.046409000 & -1.220107000 & 3.402972000 \\
\hline $\mathrm{H}$ & 4.009970000 & -2.880726000 & 0.217324000 & $\mathrm{H}$ & 4.989312000 & -1.707288000 & 3.631913000 \\
\hline $\mathrm{H}$ & 3.814279000 & -3.268805000 & 1.926552000 & C & 3.696280000 & -0.984229000 & 2.072386000 \\
\hline \multirow[t]{8}{*}{$\mathrm{H}$} & 2.402654000 & -2.822624000 & 0.958016000 & $\mathrm{H}$ & 4.366902000 & -1.292210000 & 1.276685000 \\
\hline & & & & C & 2.478167000 & -0.358816000 & 1.766149000 \\
\hline & & & & C & 2.955264000 & -1.126374000 & -1.024833000 \\
\hline & & & & C & 4.158046000 & -0.730662000 & -1.628433000 \\
\hline & & & & $\mathrm{H}$ & 4.532941000 & 0.278136000 & -1.489513000 \\
\hline & & & & C & 4.874204000 & -1.633186000 & -2.417175000 \\
\hline & & & & $\mathrm{H}$ & 5.802721000 & -1.319035000 & -2.884085000 \\
\hline & & & & C & 4.398505000 & -2.931474000 & -2.607013000 \\
\hline
\end{tabular}




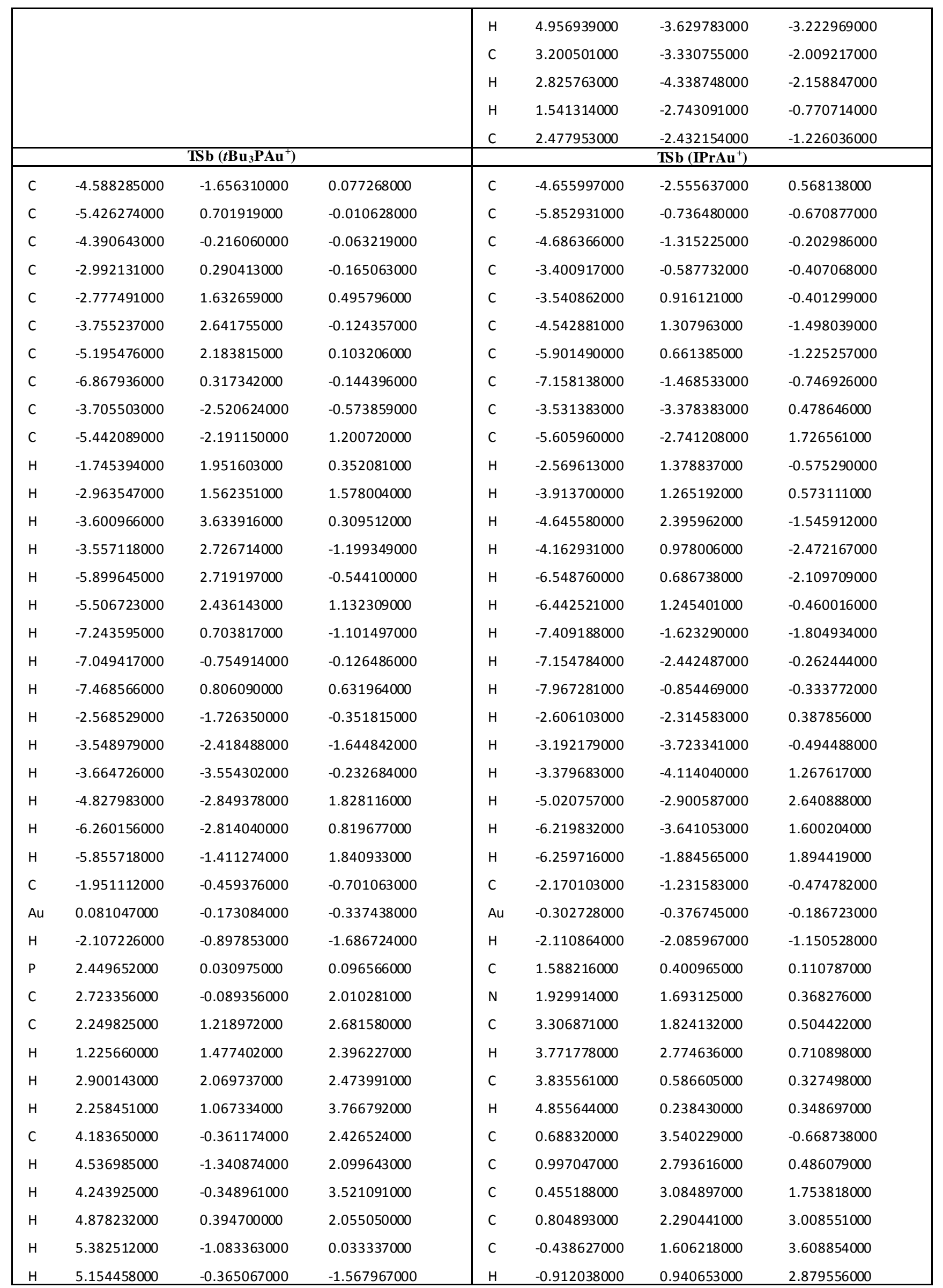




\begin{tabular}{|c|c|c|c|c|c|c|c|}
\hline $\mathrm{H}$ & 5.325343000 & -2.106677000 & -1.400373000 & $\mathrm{H}$ & -0.158492000 & 1.010486000 & 4.483879000 \\
\hline c & 4.892858000 & -1.214078000 & -0.933558000 & $\mathrm{H}$ & -1.183344000 & 2.340500000 & 3.933563000 \\
\hline c & 3.368013000 & -1.413988000 & -0.809015000 & $\mathrm{H}$ & 1.502515000 & 1.497012000 & 2.725848000 \\
\hline C & 2.757292000 & -1.585361000 & -2.221485000 & $\mathrm{H}$ & 1.803897000 & 2.574730000 & 4.923810000 \\
\hline $\mathrm{H}$ & 3.233941000 & -2.450010000 & -2.697586000 & C & 1.514810000 & 3.173107000 & 4.053793000 \\
\hline $\mathrm{H}$ & 1.682283000 & -1.783058000 & -2.172613000 & $\mathrm{H}$ & 2.419544000 & 3.631973000 & 3.642777000 \\
\hline $\mathrm{H}$ & 2.915051000 & -0.728001000 & -2.872942000 & $\mathrm{H}$ & 0.865277000 & 3.980285000 & 4.407689000 \\
\hline $\mathrm{H}$ & 0.760289000 & -1.017587000 & 2.355218000 & $\mathrm{H}$ & -0.869819000 & 4.418692000 & 2.800182000 \\
\hline $\mathrm{H}$ & 1.930542000 & -1.223768000 & 3.666114000 & C & -0.435218000 & 4.162657000 & 1.838802000 \\
\hline C & 1.814821000 & -1.208088000 & 2.576152000 & C & -0.765522000 & 4.915368000 & 0.715134000 \\
\hline $\mathrm{H}$ & 2.063555000 & -2.201957000 & 2.209137000 & $\mathrm{H}$ & -1.452964000 & 5.751371000 & 0.806103000 \\
\hline $\mathrm{H}$ & 3.599910000 & -2.802987000 & 0.900296000 & C & -0.208451000 & 4.606241000 & -0.522873000 \\
\hline H & 3.490608000 & -3.559988000 & -0.681855000 & $\mathrm{H}$ & -0.466791000 & 5.205605000 & -1.390517000 \\
\hline H & 2.029470000 & -2.923445000 & 0.074586000 & $\mathrm{H}$ & 2.905315000 & 4.705774000 & -1.876075000 \\
\hline C & 3.099132000 & -2.741985000 & -0.066780000 & C & 2.109979000 & 4.424089000 & -2.573273000 \\
\hline $\mathrm{H}$ & 4.430742000 & 2.346330000 & 1.058378000 & $\mathrm{H}$ & 1.484526000 & 5.307482000 & -2.737693000 \\
\hline c & 4.445163000 & 2.170301000 & -0.018721000 & $\mathrm{H}$ & 2.573991000 & 4.164686000 & -3.530259000 \\
\hline $\mathrm{H}$ & 4.733179000 & 3.114859000 & -0.494515000 & C & 0.199078000 & 2.814311000 & -3.047013000 \\
\hline H & 5.230768000 & 1.444202000 & -0.236200000 & $\mathrm{H}$ & -0.348221000 & 1.935345000 & -2.690956000 \\
\hline C & 3.068630000 & 1.742377000 & -0.567399000 & $\mathrm{H}$ & -0.523101000 & 3.619540000 & -3.219031000 \\
\hline C & 2.016658000 & 2.822237000 & -0.216382000 & $\mathrm{H}$ & 0.652234000 & 2.565028000 & -4.012162000 \\
\hline $\mathrm{H}$ & 1.890182000 & 2.979904000 & 0.853198000 & C & 1.286096000 & 3.235829000 & -2.039341000 \\
\hline $\mathrm{H}$ & 1.040124000 & 2.578596000 & -0.645758000 & $\mathrm{H}$ & 1.969986000 & 2.388904000 & -1.933489000 \\
\hline $\mathrm{H}$ & 2.343483000 & 3.774099000 & -0.650798000 & $\mathrm{~N}$ & 2.770616000 & -0.273087000 & 0.087427000 \\
\hline H & 2.189090000 & 1.382227000 & -2.554250000 & C & 2.917491000 & -1.694271000 & -0.150556000 \\
\hline C & 3.135843000 & 1.704199000 & -2.110211000 & C & 2.896559000 & -2.567314000 & 0.955325000 \\
\hline $\mathrm{H}$ & 3.938494000 & 1.070548000 & -2.489446000 & C & 3.100986000 & -2.139981000 & -1.474735000 \\
\hline \multirow[t]{19}{*}{$\mathrm{H}$} & 3.334996000 & 2.720935000 & -2.467053000 & C & 3.139810000 & -1.196298000 & -2.672887000 \\
\hline & & & & $\mathrm{H}$ & 3.003612000 & -0.173829000 & -2.308698000 \\
\hline & & & & $\mathrm{H}$ & 1.015248000 & -1.398936000 & -3.158459000 \\
\hline & & & & C & 1.987315000 & -1.484490000 & -3.654801000 \\
\hline & & & & $\mathrm{H}$ & 2.063526000 & -2.491378000 & -4.078281000 \\
\hline & & & & $\mathrm{H}$ & 2.010820000 & -0.772856000 & -4.486765000 \\
\hline & & & & $\mathrm{H}$ & 4.531597000 & -0.527471000 & -4.210382000 \\
\hline & & & & C & 4.504995000 & -1.248644000 & -3.387049000 \\
\hline & & & & $\mathrm{H}$ & 5.325416000 & -1.011804000 & -2.702200000 \\
\hline & & & & $\mathrm{H}$ & 4.700618000 & -2.239830000 & -3.808776000 \\
\hline & & & & $\mathrm{H}$ & 3.400941000 & -3.897232000 & -2.678792000 \\
\hline & & & & C & 3.256866000 & -3.517709000 & -1.671944000 \\
\hline & & & & $\mathrm{H}$ & 3.362855000 & -5.470464000 & -0.777139000 \\
\hline & & & & C & 3.235638000 & -4.406419000 & -0.600765000 \\
\hline & & & & $\mathrm{H}$ & 3.049735000 & -4.636323000 & 1.524328000 \\
\hline & & & & C & 3.058635000 & -3.933931000 & 0.696709000 \\
\hline & & & & $\mathrm{H}$ & 2.592431000 & -0.998893000 & 2.374767000 \\
\hline & & & & C & 2.712486000 & -2.086048000 & 2.391464000 \\
\hline & & & & C & 3.952131000 & -2.396326000 & 3.253433000 \\
\hline
\end{tabular}




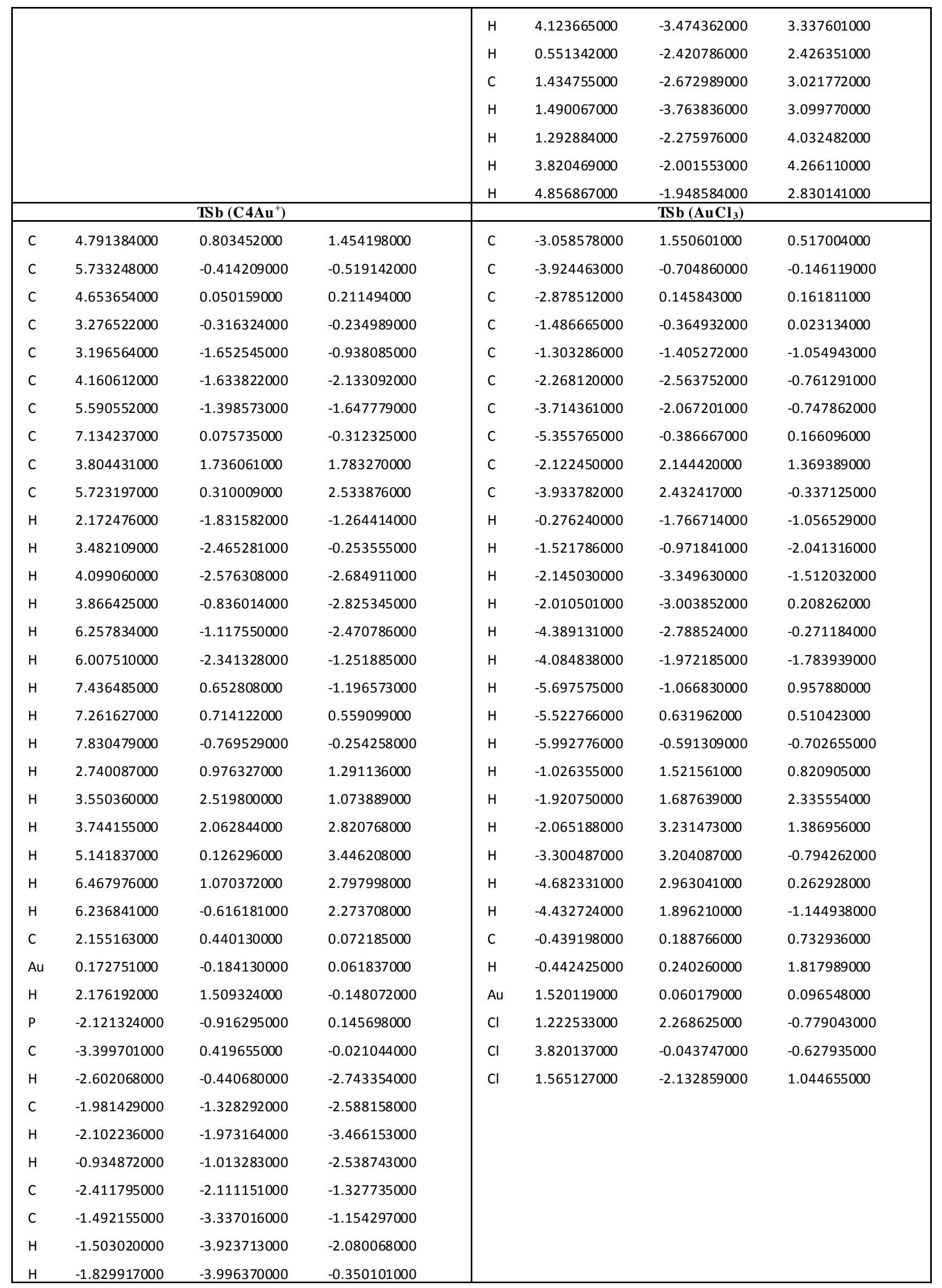




\begin{tabular}{|c|c|c|c|c|c|c|c|}
\hline $\mathrm{H}$ & -0.954637000 & -3.371113000 & 1.569297000 & & & & \\
\hline $\mathrm{H}$ & -1.257100000 & -2.938250000 & 3.252825000 & & & & \\
\hline C & -1.128824000 & -2.530214000 & 2.243593000 & & & & \\
\hline $\mathrm{H}$ & -0.455773000 & -3.048099000 & -0.952558000 & & & & \\
\hline $\mathrm{H}$ & -0.232774000 & -1.903519000 & 2.244670000 & & & & \\
\hline $\mathrm{H}$ & -2.556220000 & -0.962266000 & 3.893916000 & & & & \\
\hline C & -2.529957000 & -0.554937000 & 2.876840000 & & & & \\
\hline $\mathrm{H}$ & -1.677360000 & 0.130200000 & 2.817235000 & & & & \\
\hline $\mathrm{H}$ & -3.445488000 & 0.020519000 & 2.722003000 & & & & \\
\hline C & -2.394866000 & -1.722949000 & 1.875424000 & & & & \\
\hline C & -3.612684000 & -2.657516000 & 2.005089000 & & & & \\
\hline $\mathrm{H}$ & -3.649134000 & -3.037465000 & 3.032684000 & & & & \\
\hline $\mathrm{H}$ & -3.542192000 & -3.525754000 & 1.345339000 & & & & \\
\hline $\mathrm{H}$ & -4.562916000 & -2.153094000 & 1.822735000 & & & & \\
\hline $\mathrm{H}$ & -4.264809000 & -3.125298000 & -0.672595000 & & & & \\
\hline $\mathrm{H}$ & -3.878913000 & -3.280643000 & -2.383518000 & & & & \\
\hline $\mathrm{H}$ & -4.533936000 & -1.762420000 & -1.777158000 & & & & \\
\hline C & -3.862325000 & -2.588092000 & -1.533986000 & & & & \\
\hline H & -5.024492000 & -0.967979000 & 0.303901000 & & & & \\
\hline C & -4.757116000 & 0.063929000 & 0.123339000 & & & & \\
\hline C & -5.783849000 & 0.999713000 & 0.040521000 & & & & \\
\hline $\mathrm{H}$ & -6.815099000 & 0.681879000 & 0.158735000 & & & & \\
\hline $\mathrm{H}$ & -6.259025000 & 3.083899000 & -0.262276000 & & & & \\
\hline C & -5.473683000 & 2.337403000 & -0.193863000 & & & & \\
\hline C & -4.141987000 & 2.712086000 & -0.340672000 & & & & \\
\hline $\mathrm{H}$ & -3.894535000 & 3.752946000 & -0.525043000 & & & & \\
\hline C & -3.088973000 & 1.782119000 & -0.260273000 & & & & \\
\hline C & -1.087585000 & 3.016953000 & 0.634091000 & & & & \\
\hline C & -1.720912000 & 2.372543000 & -0.440814000 & & & & \\
\hline $\mathrm{H}$ & -1.544877000 & 2.984328000 & 1.618653000 & & & & \\
\hline C & -1.137687000 & 2.458284000 & -1.715087000 & & & & \\
\hline $\mathrm{H}$ & -1.631647000 & 1.991372000 & -2.561826000 & & & & \\
\hline C & 0.051323000 & 3.163899000 & -1.908344000 & & & & \\
\hline $\mathrm{H}$ & 0.478230000 & 3.234955000 & -2.904511000 & & & & \\
\hline C & 0.672664000 & 3.802473000 & -0.831628000 & & & & \\
\hline $\mathrm{H}$ & 1.578005000 & 4.381964000 & -0.990362000 & & & & \\
\hline C & 0.101070000 & 3.723990000 & 0.441084000 & & & & \\
\hline $\mathrm{H}$ & 0.563361000 & 4.235885000 & 1.280511000 & & & & \\
\hline \multicolumn{4}{|c|}{ Int3 $\left(\mathrm{H}_{3} \mathrm{PAu}^{+}\right)$} & \multicolumn{4}{|c|}{ Int3 $\left(\mathrm{Me}_{3} \mathrm{PAu}^{+}\right)$} \\
\hline C & 1.923197000 & 1.788974000 & -0.334881000 & C & 2.428172000 & 1.792965000 & -0.314688000 \\
\hline C & 3.271671000 & -0.092295000 & 0.631024000 & C & 3.671653000 & -0.057337000 & 0.826255000 \\
\hline C & 2.170645000 & 0.323186000 & -0.086539000 & C & 2.658149000 & 0.334351000 & -0.013658000 \\
\hline C & 1.250991000 & -0.653969000 & -0.627410000 & C & 1.811139000 & -0.663071000 & -0.649741000 \\
\hline C & 1.425362000 & -2.107509000 & -0.248011000 & C & 1.954404000 & -2.107846000 & -0.220881000 \\
\hline C & 2.904008000 & -2.483203000 & -0.127316000 & C & 3.415784000 & -2.462434000 & 0.064349000 \\
\hline C & 3.583065000 & -1.547213000 & 0.870351000 & C & 3.973464000 & -1.506694000 & 1.117235000 \\
\hline
\end{tabular}




\begin{tabular}{|c|c|c|c|c|c|c|c|}
\hline C & 4.264825000 & 0.867300000 & 1.208993000 & C & 4.585286000 & 0.920490000 & 1.502190000 \\
\hline c & 2.419639000 & 2.393200000 & -1.420007000 & C & 3.039961000 & 2.382937000 & -1.347566000 \\
\hline c & 1.109523000 & 2.521285000 & 0.707764000 & c & 1.496689000 & 2.539198000 & 0.613268000 \\
\hline $\mathrm{H}$ & 0.899434000 & -2.745088000 & -0.964020000 & $\mathrm{H}$ & 1.517507000 & -2.765775000 & -0.977369000 \\
\hline $\mathrm{H}$ & 0.941408000 & -2.263406000 & 0.727405000 & $\mathrm{H}$ & 1.370100000 & -2.251390000 & 0.699523000 \\
\hline $\mathrm{H}$ & 3.001820000 & -3.524990000 & 0.190174000 & H & 3.491864000 & -3.499640000 & 0.402887000 \\
\hline $\mathrm{H}$ & 3.390290000 & -2.397741000 & -1.106099000 & H & 4.003179000 & -2.380789000 & -0.857755000 \\
\hline $\mathrm{H}$ & 4.669962000 & -1.685602000 & 0.870644000 & $\mathrm{H}$ & 5.056689000 & -1.630418000 & 1.228536000 \\
\hline $\mathrm{H}$ & 3.259082000 & -1.787429000 & 1.896742000 & H & 3.550435000 & -1.743729000 & 2.107146000 \\
\hline $\mathrm{H}$ & 5.220617000 & 0.762944000 & 0.677718000 & $\mathrm{H}$ & 5.598191000 & 0.820008000 & 1.090017000 \\
\hline $\mathrm{H}$ & 3.955495000 & 1.909159000 & 1.146196000 & $\mathrm{H}$ & 4.276441000 & 1.958330000 & 1.387475000 \\
\hline $\mathrm{H}$ & 4.473265000 & 0.606900000 & 2.253942000 & $\mathrm{H}$ & 4.668823000 & 0.680171000 & 2.569207000 \\
\hline $\mathrm{H}$ & 0.258985000 & 0.686402000 & -1.970886000 & H & 0.951761000 & 0.648135000 & -2.110286000 \\
\hline $\mathrm{H}$ & 3.015718000 & 1.858140000 & -2.153628000 & H & 3.718762000 & 1.839436000 & -1.998355000 \\
\hline $\mathrm{H}$ & 2.248170000 & 3.449621000 & -1.606498000 & H & 2.884080000 & 3.434497000 & -1.571983000 \\
\hline $\mathrm{H}$ & 0.956305000 & 3.566587000 & 0.429181000 & $\mathrm{H}$ & 1.378697000 & 3.580484000 & 0.303314000 \\
\hline $\mathrm{H}$ & 1.604916000 & 2.495600000 & 1.686083000 & $\mathrm{H}$ & 1.873271000 & 2.527683000 & 1.643463000 \\
\hline $\mathrm{H}$ & 0.124117000 & 2.057303000 & 0.847371000 & $\mathrm{H}$ & 0.501535000 & 2.075576000 & 0.641015000 \\
\hline C & 0.202905000 & -0.298111000 & -1.509562000 & C & 0.883821000 & -0.335342000 & -1.650764000 \\
\hline $\mathrm{Au}$ & -1.520486000 & -0.108658000 & -0.190285000 & $\mathrm{Au}$ & -0.973267000 & -0.149473000 & -0.443581000 \\
\hline$P$ & -3.515971000 & 0.124146000 & 1.050791000 & $\mathrm{P}$ & -3.079690000 & 0.065528000 & 0.582816000 \\
\hline $\mathrm{H}$ & -0.156229000 & -1.094498000 & -2.163061000 & $\mathrm{H}$ & 0.561256000 & -1.143811000 & -2.306782000 \\
\hline $\mathrm{H}$ & -3.919159000 & 1.436142000 & 1.358493000 & C & -4.190289000 & -1.349756000 & 0.221834000 \\
\hline $\mathrm{H}$ & -3.564310000 & -0.486587000 & 2.317139000 & $\mathrm{H}$ & -5.162137000 & -1.204064000 & 0.703044000 \\
\hline \multirow[t]{11}{*}{$\mathrm{H}$} & -4.676935000 & -0.391558000 & 0.447279000 & $\mathrm{H}$ & -4.332563000 & -1.439335000 & -0.857884000 \\
\hline & & & & $\mathrm{H}$ & -3.740147000 & -2.274518000 & 0.590862000 \\
\hline & & & & C & -3.987014000 & 1.560381000 & 0.026830000 \\
\hline & & & & $\mathrm{H}$ & -3.408862000 & 2.454719000 & 0.271289000 \\
\hline & & & & $\mathrm{H}$ & -4.128096000 & 1.523493000 & -1.056112000 \\
\hline & & & & $\mathrm{H}$ & -4.963916000 & 1.616518000 & 0.516476000 \\
\hline & & & & C & -3.009674000 & 0.182633000 & 2.413570000 \\
\hline & & & & H & -2.411287000 & 1.048933000 & 2.705532000 \\
\hline & & & & H & -4.017963000 & 0.285302000 & 2.826175000 \\
\hline & & & & $\mathrm{H}$ & -2.543411000 & -0.717210000 & 2.821837000 \\
\hline & \multicolumn{3}{|c|}{ Int3 $\left(\mathrm{Et}_{3} \mathrm{PAu}^{+}\right)$} & \multicolumn{4}{|c|}{ Int3 $\left(\mathrm{Ph}_{3} \mathrm{PAu}^{+}\right)$} \\
\hline C & 2.852048000 & 1.816688000 & 0.118972000 & C & -3.808589000 & -1.775610000 & -0.368412000 \\
\hline C & 4.128096000 & -0.233916000 & 0.778942000 & C & -4.966506000 & 0.134895000 & 0.759347000 \\
\hline C & 3.104753000 & 0.332567000 & 0.061838000 & C & -4.017406000 & -0.302518000 & -0.128472000 \\
\hline C & 2.264106000 & -0.496650000 & -0.791830000 & c & -3.219386000 & 0.659462000 & -0.880361000 \\
\hline C & 2.405900000 & -2.000036000 & -0.688836000 & C & -3.327671000 & 2.124035000 & -0.509796000 \\
\hline C & 3.871857000 & -2.404857000 & -0.511450000 & C & -4.765453000 & 2.497914000 & -0.141066000 \\
\hline C & 4.445382000 & -1.708395000 & 0.721343000 & C & -5.248956000 & 1.598973000 & 0.995410000 \\
\hline C & 5.040604000 & 0.567121000 & 1.659470000 & C & -5.829415000 & -0.803035000 & 1.550802000 \\
\hline C & 3.437852000 & 2.648886000 & -0.749125000 & C & -4.488524000 & -2.415600000 & -1.326370000 \\
\hline C & 1.924022000 & 2.300679000 & 1.209984000 & C & -2.819119000 & -2.478559000 & 0.532810000 \\
\hline $\mathrm{H}$ & 1.954569000 & -2.478665000 & -1.562455000 & $\mathrm{H}$ & -2.944513000 & 2.743447000 & -1.325688000 \\
\hline
\end{tabular}




\begin{tabular}{|c|c|c|c|c|c|c|c|}
\hline H & 1.838768000 & -2.340214000 & 0.189660000 & H & -2.680315000 & 2.308867000 & 0.359311000 \\
\hline $\mathrm{H}$ & 3.952925000 & -3.491424000 & -0.415608000 & $\mathrm{H}$ & -4.817454000 & 3.551356000 & 0.148863000 \\
\hline $\mathrm{H}$ & 4.445120000 & -2.116609000 & -1.400491000 & $\mathrm{H}$ & -5.416187000 & 2.371845000 & -1.014485000 \\
\hline $\mathrm{H}$ & 5.531691000 & -1.841758000 & 0.778987000 & $\mathrm{H}$ & -6.322720000 & 1.731037000 & 1.171309000 \\
\hline $\mathrm{H}$ & 4.047012000 & -2.171805000 & 1.638657000 & $\mathrm{H}$ & -4.761805000 & 1.887514000 & 1.941031000 \\
\hline $\mathrm{H}$ & 6.055462000 & 0.559568000 & 1.240402000 & $\mathrm{H}$ & -6.871377000 & -0.715676000 & 1.215580000 \\
\hline $\mathrm{H}$ & 4.732585000 & 1.604827000 & 1.777917000 & H & -5.533569000 & -1.847245000 & 1.462015000 \\
\hline $\mathrm{H}$ & 5.118591000 & 0.095549000 & 2.646945000 & $\mathrm{H}$ & -5.827267000 & -0.512892000 & 2.608786000 \\
\hline $\mathrm{H}$ & 1.427534000 & 1.106911000 & -1.938300000 & H & -2.475498000 & -0.727338000 & -2.335466000 \\
\hline $\mathrm{H}$ & 4.115750000 & 2.291349000 & -1.518783000 & $\mathrm{H}$ & -5.208973000 & -1.904157000 & -1.958173000 \\
\hline $\mathrm{H}$ & 3.261727000 & 3.720394000 & -0.712076000 & $\mathrm{H}$ & -4.349005000 & -3.478249000 & -1.504933000 \\
\hline $\mathrm{H}$ & 1.773824000 & 3.381715000 & 1.152920000 & $\mathrm{H}$ & -2.726985000 & -3.535745000 & 0.271390000 \\
\hline $\mathrm{H}$ & 2.324865000 & 2.061497000 & 2.202627000 & H & -3.126340000 & -2.410390000 & 1.583694000 \\
\hline $\mathrm{H}$ & 0.942187000 & 1.812958000 & 1.146013000 & H & -1.822225000 & -2.023537000 & 0.471114000 \\
\hline C & 1.357626000 & 0.045777000 & -1.710810000 & C & -2.383672000 & 0.279589000 & -1.936188000 \\
\hline $\mathrm{Au}$ & -0.529116000 & -0.028142000 & -0.515618000 & $\mathrm{Au}$ & -0.458061000 & 0.158581000 & -0.803684000 \\
\hline$P$ & -2.669378000 & -0.025142000 & 0.464521000 & $\mathrm{H}$ & -2.092561000 & 1.051093000 & -2.648035000 \\
\hline $\mathrm{H}$ & 1.036534000 & -0.598213000 & -2.529324000 & $P$ & 1.707932000 & 0.004771000 & 0.115016000 \\
\hline C & -3.942176000 & -0.486350000 & -0.797739000 & C & 2.411896000 & 1.661220000 & 0.433126000 \\
\hline $\mathrm{H}$ & -3.818755000 & 0.221057000 & -1.624853000 & $\mathrm{H}$ & 1.591131000 & 2.489327000 & -1.390941000 \\
\hline $\mathrm{H}$ & -3.640671000 & -1.463360000 & -1.190552000 & C & 2.193262000 & 2.682208000 & -0.506961000 \\
\hline C & -3.143439000 & 1.624228000 & 1.151505000 & C & 2.750118000 & 3.944551000 & -0.311023000 \\
\hline $\mathrm{H}$ & -2.446773000 & 1.823541000 & 1.973275000 & C & 3.520155000 & 4.200423000 & 0.826793000 \\
\hline $\mathrm{H}$ & -4.140195000 & 1.526589000 & 1.596799000 & $\mathrm{H}$ & 2.579289000 & 4.728633000 & -1.042122000 \\
\hline C & -2.838319000 & -1.203701000 & 1.879290000 & $\mathrm{H}$ & 3.948374000 & 5.186016000 & 0.981270000 \\
\hline H & -2.163793000 & -0.838760000 & 2.661697000 & C & 3.735342000 & 3.191298000 & 1.766601000 \\
\hline $\mathrm{H}$ & -3.858190000 & -1.110373000 & 2.270057000 & $\mathrm{H}$ & 4.330738000 & 3.389030000 & 2.652553000 \\
\hline C & -2.518022000 & -2.661668000 & 1.526529000 & C & 3.184747000 & 1.922739000 & 1.574521000 \\
\hline $\mathrm{H}$ & -3.207948000 & -3.063142000 & 0.778713000 & $\mathrm{H}$ & 3.354372000 & 1.144874000 & 2.311723000 \\
\hline $\mathrm{H}$ & -1.499910000 & -2.764160000 & 1.138030000 & C & 0.700339000 & -0.673945000 & 2.630430000 \\
\hline H & -2.601480000 & -3.287386000 & 2.419288000 & H & -0.103383000 & 0.017637000 & 2.391438000 \\
\hline C & -5.397333000 & -0.514113000 & -0.310728000 & C & 0.709919000 & -1.327426000 & 3.861195000 \\
\hline $\mathrm{H}$ & -5.729949000 & 0.464352000 & 0.046904000 & H & -0.084573000 & -1.141695000 & 4.577392000 \\
\hline $\mathrm{H}$ & -6.053299000 & -0.799350000 & -1.138074000 & C & 1.736398000 & -2.224633000 & 4.168905000 \\
\hline $\mathrm{H}$ & -5.549034000 & -1.241428000 & 0.491793000 & $\mathrm{H}$ & 1.739126000 & -2.738165000 & 5.125401000 \\
\hline C & -3.109601000 & 2.764552000 & 0.125531000 & C & 2.754974000 & -2.464154000 & 3.245543000 \\
\hline $\mathrm{H}$ & -3.834027000 & 2.612162000 & -0.679811000 & $\mathrm{H}$ & 3.551293000 & -3.163312000 & 3.481095000 \\
\hline $\mathrm{H}$ & -3.356699000 & 3.711085000 & 0.613843000 & C & 2.754865000 & -1.808541000 & 2.012821000 \\
\hline \multirow[t]{8}{*}{$\mathrm{H}$} & -2.117703000 & 2.868596000 & -0.325504000 & $\mathrm{H}$ & 3.549086000 & -2.002521000 & 1.299576000 \\
\hline & & & & C & 1.727110000 & -0.906301000 & 1.700204000 \\
\hline & & & & C & 2.843138000 & -0.860321000 & -1.025730000 \\
\hline & & & & C & 4.180946000 & -0.460008000 & -1.165553000 \\
\hline & & & & $\mathrm{H}$ & 4.556755000 & 0.397189000 & -0.616535000 \\
\hline & & & & C & 5.031940000 & -1.163303000 & -2.019736000 \\
\hline & & & & $\mathrm{H}$ & 6.064991000 & -0.847614000 & -2.127275000 \\
\hline & & & & C & 4.556839000 & -2.263633000 & -2.735209000 \\
\hline
\end{tabular}




\begin{tabular}{|c|c|c|c|c|c|c|c|}
\hline & & & & $\mathrm{H}$ & 5.221223000 & -2.805423000 & -3.401275000 \\
\hline & & & & C & 3.225024000 & -2.664229000 & -2.600879000 \\
\hline & & & & $\mathrm{H}$ & 2.851947000 & -3.515836000 & -3.161192000 \\
\hline & & & & $\mathrm{H}$ & 1.330983000 & -2.274539000 & -1.658582000 \\
\hline & & & & C & 2.368227000 & -1.964327000 & -1.753263000 \\
\hline & & Int3 $\left(t \mathrm{Bu}_{3} \mathrm{PAu}^{+}\right.$ & & & & Int3 $\left(\operatorname{IPrAu} u^{+}\right)$ & \\
\hline C & 3.481505000 & 1.785416000 & -0.296671000 & C & -2.643881000 & -3.402553000 & -0.089704000 \\
\hline c & 4.646476000 & -0.107705000 & 0.849701000 & c & -4.630482000 & -1.890254000 & -0.220141000 \\
\hline C & 3.679112000 & 0.317186000 & -0.023816000 & C & -3.344856000 & -2.169692000 & -0.598401000 \\
\hline C & 2.848016000 & -0.653328000 & -0.728710000 & c & -2.649194000 & -1.298847000 & -1.546866000 \\
\hline c & 2.949236000 & -2.108155000 & -0.319737000 & c & -3.320265000 & 0.002218000 & -1.941486000 \\
\hline C & 4.392200000 & -2.490566000 & 0.018825000 & C & -4.834901000 & -0.173299000 & -2.073056000 \\
\hline c & 4.919169000 & -1.568419000 & 1.116660000 & c & -5.398789000 & -0.705517000 & -0.756986000 \\
\hline C & 5.541998000 & 0.840080000 & 1.592161000 & C & -5.402856000 & -2.755483000 & 0.733035000 \\
\hline C & 4.144279000 & 2.396417000 & -1.284955000 & C & -2.733080000 & -4.561804000 & -0.751409000 \\
\hline C & 2.519764000 & 2.517455000 & 0.610698000 & C & -1.864597000 & -3.260894000 & 1.196628000 \\
\hline H & 2.536359000 & -2.745896000 & -1.106605000 & H & -2.874743000 & 0.382385000 & -2.865301000 \\
\hline H & 2.325180000 & -2.260318000 & 0.572682000 & H & -3.118641000 & 0.749240000 & -1.161481000 \\
\hline $\mathrm{H}$ & 4.440349000 & -3.536719000 & 0.334747000 & $\mathrm{H}$ & -5.301822000 & 0.780085000 & -2.337494000 \\
\hline H & 5.018781000 & -2.395371000 & -0.875945000 & H & -5.059470000 & -0.877288000 & -2.883336000 \\
\hline $\mathrm{H}$ & 5.996301000 & -1.708541000 & 1.263533000 & $\mathrm{H}$ & -6.453100000 & -0.984866000 & -0.866180000 \\
\hline $\mathrm{H}$ & 4.458119000 & -1.826425000 & 2.083846000 & $\mathrm{H}$ & -5.383424000 & 0.086389000 & 0.009034000 \\
\hline H & 6.573137000 & 0.731925000 & 1.230589000 & H & -6.235340000 & -3.237526000 & 0.203860000 \\
\hline H & 5.255744000 & 1.885247000 & 1.484577000 & H & -4.801537000 & -3.536307000 & 1.196517000 \\
\hline $\mathrm{H}$ & 5.566028000 & 0.577870000 & 2.657094000 & $\mathrm{H}$ & -5.858855000 & -2.136582000 & 1.515718000 \\
\hline $\mathrm{H}$ & 2.081271000 & 0.700567000 & -2.202438000 & H & -1.051575000 & -2.656975000 & -1.995507000 \\
\hline $\mathrm{H}$ & 4.845714000 & 1.863401000 & -1.920236000 & $\mathrm{H}$ & -3.308531000 & -4.656465000 & -1.667723000 \\
\hline $\mathrm{H}$ & 4.009864000 & 3.455755000 & -1.485811000 & H & -2.234906000 & -5.458012000 & -0.391847000 \\
\hline $\mathrm{H}$ & 2.410470000 & 3.563781000 & 0.314145000 & H & -1.369813000 & -4.197568000 & 1.466109000 \\
\hline H & 2.862838000 & 2.489711000 & 1.652428000 & $\mathrm{H}$ & -2.522637000 & -2.969730000 & 2.024678000 \\
\hline $\mathrm{H}$ & 1.527444000 & 2.049025000 & 0.597341000 & $\mathrm{H}$ & -1.100627000 & -2.477367000 & 1.115395000 \\
\hline C & 1.992238000 & -0.294024000 & -1.772990000 & C & -1.426873000 & -1.645610000 & -2.124444000 \\
\hline $\mathrm{Au}$ & 0.047801000 & -0.138255000 & -0.644295000 & $\mathrm{Au}$ & -0.191855000 & -0.432846000 & -0.704985000 \\
\hline H & 1.677502000 & -1.083414000 & -2.454746000 & $\mathrm{H}$ & -1.140955000 & -1.139441000 & -3.045194000 \\
\hline$P$ & -2.163227000 & 0.013392000 & 0.224841000 & C & 1.139435000 & 0.682237000 & 0.360943000 \\
\hline C & -2.499487000 & 1.851420000 & 0.728695000 & N & 0.910089000 & 1.893503000 & 0.933660000 \\
\hline C & -1.751807000 & 2.174722000 & 2.040222000 & C & 2.071776000 & 2.381270000 & 1.517459000 \\
\hline $\mathrm{H}$ & -0.686269000 & 1.935116000 & 1.978668000 & $\mathrm{H}$ & 2.097412000 & 3.332097000 & 2.024887000 \\
\hline H & -2.176699000 & 1.672246000 & 2.909831000 & C & 3.039502000 & 1.454164000 & 1.299080000 \\
\hline H & -1.834127000 & 3.252119000 & 2.219659000 & H & 4.081042000 & 1.433761000 & 1.575835000 \\
\hline C & -3.998372000 & 2.167950000 & 0.917611000 & C & -0.621025000 & 3.489109000 & -0.133892000 \\
\hline $\mathrm{H}$ & -4.564202000 & 2.094485000 & -0.012953000 & C & -0.355194000 & 2.599865000 & 0.926900000 \\
\hline $\mathrm{H}$ & -4.091619000 & 3.202414000 & 1.266912000 & C & -1.250347000 & 2.386572000 & 1.994586000 \\
\hline H & -4.476142000 & 1.529824000 & 1.663067000 & C & -0.939287000 & 1.459328000 & 3.165702000 \\
\hline $\mathrm{H}$ & -5.257122000 & -0.038654000 & -0.172498000 & C & -2.028534000 & 0.387391000 & 3.357405000 \\
\hline $\mathrm{H}$ & -4.808999000 & -1.745571000 & -0.028647000 & $\mathrm{H}$ & -2.179413000 & -0.194042000 & 2.442335000 \\
\hline
\end{tabular}




\begin{tabular}{|c|c|c|c|c|c|c|c|}
\hline $\mathrm{H}$ & -5.409482000 & -1.116746000 & -1.556813000 & $\mathrm{H}$ & -1.741122000 & -0.302566000 & 4.157279000 \\
\hline c & -4.785534000 & -0.876634000 & -0.688643000 & $\mathrm{H}$ & -2.988990000 & 0.831340000 & 3.638430000 \\
\hline c & -3.362452000 & -0.550532000 & -1.188600000 & $\mathrm{H}$ & -0.005553000 & 0.932988000 & 2.945581000 \\
\hline C & -2.771078000 & -1.794916000 & -1.893990000 & $\mathrm{H}$ & -0.458967000 & 1.591463000 & 5.288337000 \\
\hline $\mathrm{H}$ & -3.447626000 & -2.075105000 & -2.709229000 & C & -0.720135000 & 2.262606000 & 4.463896000 \\
\hline $\mathrm{H}$ & -1.794047000 & -1.580200000 & -2.336907000 & $\mathrm{H}$ & 0.087051000 & 2.993538000 & 4.353359000 \\
\hline H & -2.668572000 & -2.663130000 & -1.245722000 & $\mathrm{H}$ & -1.624405000 & 2.808790000 & 4.751279000 \\
\hline $\mathrm{H}$ & -0.834766000 & 2.642231000 & -0.474187000 & $\mathrm{H}$ & -3.171449000 & 2.961502000 & 2.774596000 \\
\hline $\mathrm{H}$ & -2.067586000 & 3.824413000 & -0.023496000 & C & -2.459581000 & 3.092700000 & 1.965732000 \\
\hline C & -1.911417000 & 2.790565000 & -0.351531000 & C & -2.759186000 & 3.967943000 & 0.924840000 \\
\hline $\mathrm{H}$ & -2.382286000 & 2.684871000 & -1.326720000 & $\mathrm{H}$ & -3.700991000 & 4.508742000 & 0.926401000 \\
\hline $\mathrm{H}$ & -3.982153000 & 1.442814000 & -1.926650000 & C & -1.848844000 & 4.163032000 & -0.110392000 \\
\hline H & -4.003450000 & 0.151366000 & -3.118222000 & $\mathrm{H}$ & -2.088789000 & 4.857924000 & -0.909073000 \\
\hline H & -2.459983000 & 0.850879000 & -2.631134000 & $\mathrm{H}$ & 1.269759000 & 5.514015000 & -0.324595000 \\
\hline C & -3.447461000 & 0.554286000 & -2.264822000 & C & 0.822557000 & 5.225318000 & -1.280902000 \\
\hline $\mathrm{H}$ & -3.529043000 & 0.072264000 & 3.107731000 & $\mathrm{H}$ & -0.013316000 & 5.904834000 & -1.475129000 \\
\hline c & -3.541194000 & -0.906884000 & 2.625222000 & $\mathrm{H}$ & 1.568481000 & 5.384302000 & -2.066016000 \\
\hline $\mathrm{H}$ & -3.567013000 & -1.655926000 & 3.424851000 & C & -0.219209000 & 3.343214000 & -2.635162000 \\
\hline H & -4.471552000 & -1.002490000 & 2.062582000 & $\mathrm{H}$ & -0.498198000 & 2.284358000 & -2.645145000 \\
\hline C & -2.289175000 & -1.162728000 & 1.759501000 & $\mathrm{H}$ & -1.109505000 & 3.929398000 & -2.885352000 \\
\hline C & -1.020995000 & -1.002225000 & 2.631092000 & $\mathrm{H}$ & 0.517755000 & 3.508169000 & -3.427710000 \\
\hline $\mathrm{H}$ & -0.907887000 & -0.009935000 & 3.063054000 & C & 0.364374000 & 3.753102000 & -1.269246000 \\
\hline $\mathrm{H}$ & -0.113572000 & -1.235822000 & 2.065881000 & $\mathrm{H}$ & 1.254732000 & 3.139427000 & -1.101594000 \\
\hline $\mathrm{H}$ & -1.087640000 & -1.714926000 & 3.460753000 & $\mathrm{~N}$ & 2.451552000 & 0.415557000 & 0.588914000 \\
\hline H & -1.451593000 & -2.859427000 & 0.632173000 & C & 3.167332000 & -0.766522000 & 0.147631000 \\
\hline C & -2.301242000 & -2.631547000 & 1.282898000 & C & 3.319909000 & -1.835126000 & 1.054782000 \\
\hline $\mathrm{H}$ & -3.223303000 & -2.908590000 & 0.770867000 & C & 3.718216000 & -0.773133000 & -1.149888000 \\
\hline \multirow[t]{19}{*}{$\mathrm{H}$} & -2.218891000 & -3.274832000 & 2.165746000 & C & 3.599044000 & 0.404101000 & -2.113811000 \\
\hline & & & & $\mathrm{H}$ & 2.928009000 & 1.145369000 & -1.669380000 \\
\hline & & & & $\mathrm{H}$ & 1.998896000 & -0.478538000 & -3.320714000 \\
\hline & & & & C & 2.979230000 & -0.011863000 & -3.461220000 \\
\hline & & & & $\mathrm{H}$ & 3.614207000 & -0.722633000 & -3.999241000 \\
\hline & & & & $\mathrm{H}$ & 2.852284000 & 0.865372000 & -4.103778000 \\
\hline & & & & $\mathrm{H}$ & 4.862101000 & 1.956761000 & -2.978795000 \\
\hline & & & & C & 4.966085000 & 1.087505000 & -2.321465000 \\
\hline & & & & $\mathrm{H}$ & 5.393151000 & 1.428474000 & -1.373043000 \\
\hline & & & & $\mathrm{H}$ & 5.686160000 & 0.404357000 & -2.783274000 \\
\hline & & & & $\mathrm{H}$ & 4.873926000 & -1.957782000 & -2.524258000 \\
\hline & & & & C & 4.433215000 & -1.914726000 & -1.533427000 \\
\hline & & & & $\mathrm{H}$ & 5.162570000 & -3.860377000 & -0.981525000 \\
\hline & & & & C & 4.598564000 & -2.988602000 & -0.663724000 \\
\hline & & & & $\mathrm{H}$ & 4.194603000 & -3.786790000 & 1.285688000 \\
\hline & & & & C & 4.050183000 & -2.945850000 & 0.615365000 \\
\hline & & & & $\mathrm{H}$ & 2.067317000 & -0.960437000 & 2.548651000 \\
\hline & & & & C & 2.764002000 & -1.801761000 & 2.475773000 \\
\hline & & & & C & 3.892767000 & -1.563483000 & 3.500481000 \\
\hline
\end{tabular}




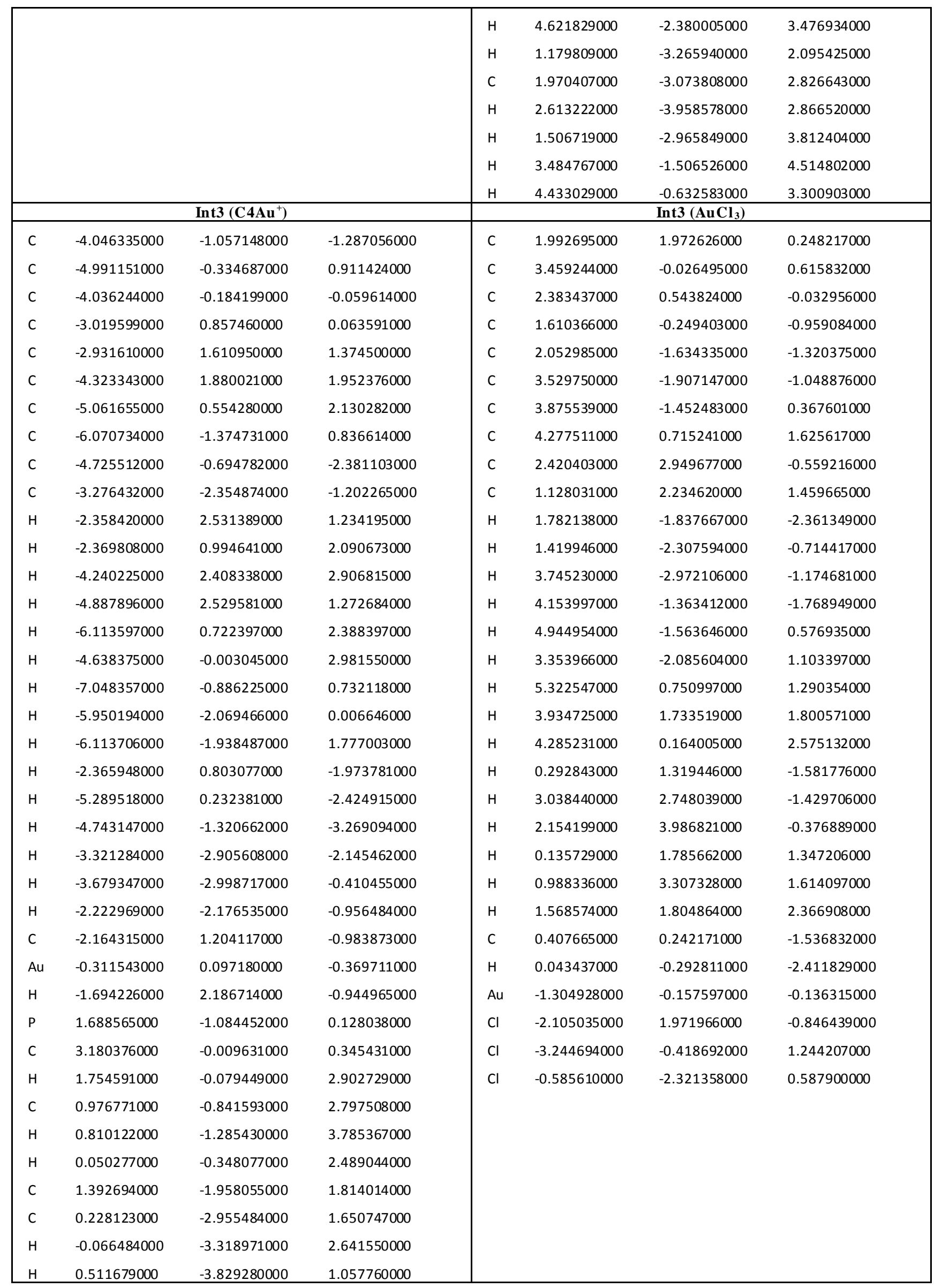




\begin{tabular}{|c|c|c|c|c|c|c|c|}
\hline $\mathrm{H}$ & 0.229635000 & -3.487239000 & -1.117390000 & & & & \\
\hline $\mathrm{H}$ & 0.942250000 & -3.489201000 & -2.730102000 & & & & \\
\hline C & 0.728994000 & -2.859988000 & -1.858756000 & & & & \\
\hline $\mathrm{H}$ & -0.650078000 & -2.491617000 & 1.190711000 & & & & \\
\hline $\mathrm{H}$ & 0.032232000 & -2.078769000 & -2.175077000 & & & & \\
\hline $\mathrm{H}$ & 2.793625000 & -2.010513000 & -3.359507000 & & & & \\
\hline C & 2.663196000 & -1.392236000 & -2.464567000 & & & & \\
\hline $\mathrm{H}$ & 2.001455000 & -0.561946000 & -2.732354000 & & & & \\
\hline $\mathrm{H}$ & 3.639712000 & -0.984826000 & -2.193205000 & & & & \\
\hline C & 2.058355000 & -2.266225000 & -1.345113000 & & & & \\
\hline C & 3.018599000 & -3.426271000 & -1.018684000 & & & & \\
\hline $\mathrm{H}$ & 3.178491000 & -4.008116000 & -1.933335000 & & & & \\
\hline $\mathrm{H}$ & 2.606889000 & -4.110083000 & -0.272106000 & & & & \\
\hline $\mathrm{H}$ & 4.000350000 & -3.086220000 & -0.684093000 & & & & \\
\hline $\mathrm{H}$ & 3.017961000 & -3.461245000 & 1.756361000 & & & & \\
\hline $\mathrm{H}$ & 2.283685000 & -3.190908000 & 3.333059000 & & & & \\
\hline $\mathrm{H}$ & 3.408938000 & -2.001412000 & 2.688456000 & & & & \\
\hline C & 2.609365000 & -2.691373000 & 2.413699000 & & & & \\
\hline H & 4.437886000 & -1.723896000 & 0.748333000 & & & & \\
\hline C & 4.398463000 & -0.646734000 & 0.664726000 & & & & \\
\hline C & 5.571875000 & 0.067040000 & 0.888195000 & & & & \\
\hline $\mathrm{H}$ & 6.488046000 & -0.462692000 & 1.129376000 & & & & \\
\hline $\mathrm{H}$ & 6.456527000 & 2.033986000 & 0.980286000 & & & & \\
\hline C & 5.553993000 & 1.457323000 & 0.803258000 & & & & \\
\hline C & 4.367336000 & 2.105184000 & 0.475595000 & & & & \\
\hline $\mathrm{H}$ & 4.353529000 & 3.187012000 & 0.387780000 & & & & \\
\hline C & 3.172482000 & 1.403765000 & 0.229289000 & & & & \\
\hline C & 1.737245000 & 2.482859000 & -1.539205000 & & & & \\
\hline C & 2.017479000 & 2.270383000 & -0.178098000 & & & & \\
\hline $\mathrm{H}$ & 2.284538000 & 1.921597000 & -2.290558000 & & & & \\
\hline C & 1.338393000 & 3.043193000 & 0.777879000 & & & & \\
\hline $\mathrm{H}$ & 1.568339000 & 2.911770000 & 1.831262000 & & & & \\
\hline C & 0.401307000 & 4.001073000 & 0.383027000 & & & & \\
\hline $\mathrm{H}$ & -0.097978000 & 4.606157000 & 1.134424000 & & & & \\
\hline C & 0.135166000 & 4.207952000 & -0.973309000 & & & & \\
\hline $\mathrm{H}$ & -0.570734000 & 4.973842000 & -1.280718000 & & & & \\
\hline C & 0.803200000 & 3.443309000 & -1.933246000 & & & & \\
\hline $\mathrm{H}$ & 0.616119000 & 3.609906000 & -2.989944000 & & & & \\
\hline \multicolumn{4}{|c|}{ TSc $\left(\mathbf{H}_{3} \mathbf{P A u}^{+}\right)$} & \multicolumn{4}{|c|}{$\mathbf{T S c}\left(\mathrm{Me}_{3} \mathbf{P A u}^{+}\right)$} \\
\hline C & -2.010453000 & 1.598203000 & -0.024581000 & C & 2.685955000 & -1.586664000 & -0.003414000 \\
\hline C & -3.838688000 & -0.250626000 & 0.061162000 & C & 4.414529000 & 0.347121000 & 0.076506000 \\
\hline C & -2.564410000 & 0.189834000 & 0.010147000 & C & 3.163263000 & -0.157589000 & 0.000112000 \\
\hline C & -1.390651000 & -0.657319000 & 0.035079000 & C & 1.947471000 & 0.628221000 & -0.005009000 \\
\hline C & -1.535087000 & -2.133769000 & 0.243812000 & C & 2.015965000 & 2.105188000 & 0.237019000 \\
\hline C & -2.876590000 & -2.591816000 & -0.363656000 & C & 3.339582000 & 2.640892000 & -0.344528000 \\
\hline C & -4.062480000 & -1.750724000 & 0.140178000 & C & 4.560109000 & 1.855325000 & 0.166144000 \\
\hline
\end{tabular}




\begin{tabular}{|c|c|c|c|c|c|c|c|}
\hline c & -5.062519000 & 0.618331000 & 0.094224000 & C & 5.680380000 & -0.456807000 & 0.147915000 \\
\hline c & -2.254227000 & 2.433747000 & -1.251085000 & C & 3.022177000 & -2.464931000 & -1.173959000 \\
\hline c & -1.852704000 & 2.326784000 & 1.280749000 & c & 2.446412000 & -2.264005000 & 1.314063000 \\
\hline C & -0.279304000 & 0.091988000 & -0.118878000 & C & 0.871222000 & -0.167111000 & -0.206397000 \\
\hline $\mathrm{H}$ & -0.691943000 & -2.679885000 & -0.190620000 & $\mathrm{H}$ & 1.152160000 & 2.615556000 & -0.200575000 \\
\hline $\mathrm{H}$ & -1.526207000 & -2.337761000 & 1.323726000 & $\mathrm{H}$ & 1.982451000 & 2.291865000 & 1.319950000 \\
\hline $\mathrm{H}$ & -3.047769000 & -3.646753000 & -0.131410000 & H & 3.455456000 & 3.700350000 & -0.098398000 \\
\hline $\mathrm{H}$ & -2.819379000 & -2.511041000 & -1.455397000 & $\mathrm{H}$ & 3.301701000 & 2.570208000 & -1.437944000 \\
\hline $\mathrm{H}$ & -4.963402000 & -2.002873000 & -0.432168000 & H & 5.454908000 & 2.156642000 & -0.392357000 \\
\hline $\mathrm{H}$ & -4.298650000 & -2.007709000 & 1.184061000 & $\mathrm{H}$ & 4.771223000 & 2.115502000 & 1.214806000 \\
\hline $\mathrm{H}$ & -5.676303000 & 0.449762000 & -0.799146000 & $\mathrm{H}$ & 6.307798000 & -0.261779000 & -0.730564000 \\
\hline $\mathrm{H}$ & -4.841333000 & 1.686103000 & 0.159327000 & $\mathrm{H}$ & 5.513879000 & -1.534187000 & 0.215865000 \\
\hline $\mathrm{H}$ & -5.690696000 & 0.354292000 & 0.953651000 & H & 6.271897000 & -0.155156000 & 1.021052000 \\
\hline H & -1.626574000 & 3.328128000 & -1.278926000 & H & 2.428772000 & -3.382627000 & -1.191915000 \\
\hline $\mathrm{H}$ & -2.134202000 & 1.854975000 & -2.168957000 & H & 2.931456000 & -1.933653000 & -2.123513000 \\
\hline $\mathrm{H}$ & -3.301239000 & 2.767164000 & -1.209383000 & $\mathrm{H}$ & 4.076934000 & -2.758282000 & -1.065650000 \\
\hline $\mathrm{H}$ & -1.147081000 & 3.158661000 & 1.205881000 & $\mathrm{H}$ & 1.733640000 & -3.088996000 & 1.227779000 \\
\hline $\mathrm{H}$ & -2.834075000 & 2.756303000 & 1.529812000 & $\mathrm{H}$ & 3.405340000 & -2.702111000 & 1.630187000 \\
\hline $\mathrm{H}$ & -1.563240000 & 1.657422000 & 2.091724000 & H & 2.121418000 & -1.565338000 & 2.085145000 \\
\hline $\mathrm{H}$ & -0.635392000 & 1.213942000 & -0.339432000 & $\mathrm{H}$ & 1.247210000 & -1.236937000 & -0.495388000 \\
\hline $\mathrm{H}$ & 4.862655000 & 0.960496000 & -0.237243000 & $P$ & -3.530192000 & 0.124677000 & 0.062584000 \\
\hline $\mathrm{H}$ & 4.681041000 & -0.569774000 & 1.291560000 & $\mathrm{Au}$ & -1.161221000 & -0.089059000 & -0.077039000 \\
\hline $\mathrm{H}$ & 4.702663000 & -1.128356000 & -0.805582000 & C & -4.434291000 & -1.473178000 & -0.027386000 \\
\hline$P$ & 4.111501000 & -0.191393000 & 0.061847000 & $\mathrm{H}$ & -4.116473000 & -2.122317000 & 0.792172000 \\
\hline \multirow[t]{11}{*}{$\mathrm{Au}$} & 1.737231000 & 0.004051000 & -0.028634000 & $\mathrm{H}$ & -5.513808000 & -1.308629000 & 0.041104000 \\
\hline & & & & $\mathrm{H}$ & -4.205335000 & -1.970963000 & -0.972803000 \\
\hline & & & & C & -4.102556000 & 0.910401000 & 1.621951000 \\
\hline & & & & $\mathrm{H}$ & -3.778787000 & 0.310220000 & 2.475783000 \\
\hline & & & & $\mathrm{H}$ & -3.660900000 & 1.905859000 & 1.712752000 \\
\hline & & & & $\mathrm{H}$ & -5.193136000 & 0.997436000 & 1.634165000 \\
\hline & & & & C & -4.249270000 & 1.154426000 & -1.278555000 \\
\hline & & & & $\mathrm{H}$ & -5.334931000 & 1.231109000 & -1.166592000 \\
\hline & & & & H & -3.812424000 & 2.155545000 & -1.245796000 \\
\hline & & & & $\mathrm{H}$ & -4.016779000 & 0.708479000 & -2.248693000 \\
\hline & \multicolumn{3}{|c|}{$\mathbf{T S c}\left(\mathrm{Et}_{3} \mathbf{P A u}^{+}\right)$} & \multicolumn{4}{|c|}{ TSc $\left(\mathrm{Ph}_{3} \mathrm{PAu}^{+}\right)$} \\
\hline C & 3.189224000 & -1.568141000 & -0.092037000 & C & 4.283191000 & 1.618889000 & 0.005798000 \\
\hline C & 4.881359000 & 0.371025000 & 0.217591000 & C & 5.972660000 & -0.348126000 & -0.056337000 \\
\hline C & 3.640613000 & -0.140699000 & 0.055824000 & C & 4.732303000 & 0.182740000 & 0.028152000 \\
\hline C & 2.412482000 & 0.625319000 & 0.088706000 & c & 3.501692000 & -0.578554000 & 0.060238000 \\
\hline C & 2.451325000 & 2.068652000 & 0.489054000 & C & 3.537797000 & -2.058694000 & -0.169903000 \\
\hline C & 3.779194000 & 2.681514000 & 0.000992000 & C & 4.856349000 & -2.615218000 & 0.403155000 \\
\hline C & 5.000550000 & 1.864787000 & 0.458391000 & C & 6.087313000 & -1.859766000 & -0.127895000 \\
\hline C & 6.157812000 & -0.418555000 & 0.250504000 & C & 7.253105000 & 0.429071000 & -0.158082000 \\
\hline C & 3.580843000 & -2.337264000 & -1.320125000 & C & 4.655165000 & 2.520838000 & 1.146928000 \\
\hline C & 2.898128000 & -2.360453000 & 1.147921000 & C & 4.019843000 & 2.267745000 & -1.321131000 \\
\hline C & 1.354470000 & -0.156782000 & -0.232907000 & C & 2.442244000 & 0.239026000 & 0.268331000 \\
\hline
\end{tabular}




\begin{tabular}{|c|c|c|c|c|c|c|c|}
\hline H & 1.589512000 & 2.611167000 & 0.087909000 & H & 2.668273000 & -2.547068000 & 0.281114000 \\
\hline $\mathrm{H}$ & 2.390799000 & 2.138145000 & 1.584688000 & $\mathrm{H}$ & 3.488598000 & -2.255533000 & -1.250561000 \\
\hline $\mathrm{H}$ & 3.871660000 & 3.711024000 & 0.358981000 & $\mathrm{H}$ & 4.949073000 & -3.679426000 & 0.167624000 \\
\hline $\mathrm{H}$ & 3.768760000 & 2.725304000 & -1.094484000 & $\mathrm{H}$ & 4.831317000 & -2.532000000 & 1.496105000 \\
\hline $\mathrm{H}$ & 5.902415000 & 2.231809000 & -0.047091000 & H & 6.981497000 & -2.172016000 & 0.425776000 \\
\hline $\mathrm{H}$ & 5.186149000 & 2.022445000 & 1.532067000 & $\mathrm{H}$ & 6.283491000 & -2.136835000 & -1.175212000 \\
\hline $\mathrm{H}$ & 6.805432000 & -0.134920000 & -0.588347000 & $\mathrm{H}$ & 7.890389000 & 0.232915000 & 0.713058000 \\
\hline $\mathrm{H}$ & 6.007435000 & -1.499796000 & 0.214965000 & $\mathrm{H}$ & 7.106808000 & 1.508538000 & -0.237586000 \\
\hline $\mathrm{H}$ & 6.720655000 & -0.190188000 & 1.163892000 & $\mathrm{H}$ & 7.824823000 & 0.104536000 & -1.036262000 \\
\hline $\mathrm{H}$ & 3.006440000 & -3.259875000 & -1.435159000 & H & 4.072055000 & 3.445333000 & 1.154940000 \\
\hline $\mathrm{H}$ & 3.510257000 & -1.728876000 & -2.224202000 & $\mathrm{H}$ & 4.577889000 & 2.014280000 & 2.111101000 \\
\hline $\mathrm{H}$ & 4.637503000 & -2.620318000 & -1.202758000 & $\mathrm{H}$ & 5.710469000 & 2.800523000 & 1.010603000 \\
\hline $\mathrm{H}$ & 2.195462000 & -3.176808000 & 0.957405000 & $\mathrm{H}$ & 3.308482000 & 3.094478000 & -1.238638000 \\
\hline $\mathrm{H}$ & 3.845245000 & -2.823380000 & 1.465035000 & $\mathrm{H}$ & 4.971992000 & 2.699582000 & -1.665609000 \\
\hline H & 2.534909000 & -1.734783000 & 1.963218000 & $\mathrm{H}$ & 3.677821000 & 1.553748000 & -2.070344000 \\
\hline H & 1.748718000 & -1.178719000 & -0.628842000 & $\mathrm{H}$ & 2.831363000 & 1.297424000 & 0.557425000 \\
\hline $\mathrm{Au}$ & -0.685971000 & -0.112118000 & -0.165586000 & $\mathrm{Au}$ & 0.408682000 & 0.176483000 & 0.136939000 \\
\hline$P$ & -3.065827000 & 0.039392000 & -0.110012000 & $\mathrm{P}$ & -1.969813000 & -0.025991000 & -0.005434000 \\
\hline $\mathrm{H}$ & -3.631225000 & -1.705242000 & -1.653535000 & C & -2.759506000 & -0.179869000 & 1.638729000 \\
\hline C & -3.914045000 & -1.528465000 & -0.609876000 & C & -2.738849000 & 1.425460000 & -0.816701000 \\
\hline $\mathrm{H}$ & -4.995199000 & -1.349505000 & -0.599105000 & H & -4.155970000 & 0.306610000 & -2.006711000 \\
\hline $\mathrm{H}$ & -3.865457000 & -2.609745000 & 1.296379000 & C & -3.788215000 & 1.291245000 & -1.737312000 \\
\hline C & -3.550684000 & -2.740961000 & 0.256806000 & C & -2.267377000 & 2.706743000 & -0.485895000 \\
\hline $\mathrm{H}$ & -2.471865000 & -2.926331000 & 0.250668000 & $\mathrm{H}$ & -1.449744000 & 2.818345000 & 0.221636000 \\
\hline H & -4.047581000 & -3.636926000 & -0.125631000 & C & -2.846793000 & 3.836847000 & -1.059876000 \\
\hline C & -3.735641000 & 1.314400000 & -1.272385000 & $\mathrm{H}$ & -2.479683000 & 4.824307000 & -0.797222000 \\
\hline H & -3.466704000 & 0.976103000 & -2.279238000 & H & -4.339710000 & 4.578438000 & -2.427195000 \\
\hline $\mathrm{H}$ & -4.829593000 & 1.292476000 & -1.210809000 & C & -3.892960000 & 3.697814000 & -1.975863000 \\
\hline $\mathrm{H}$ & -5.624017000 & 1.389033000 & 1.100172000 & C & -4.360515000 & 2.427064000 & -2.313673000 \\
\hline C & -5.212672000 & 0.597427000 & 1.732971000 & $\mathrm{H}$ & -5.171216000 & 2.316229000 & -3.027265000 \\
\hline C & -3.691351000 & 0.471149000 & 1.580546000 & C & -2.090183000 & -0.891357000 & 2.647704000 \\
\hline H & -3.291858000 & -0.293860000 & 2.255240000 & C & -2.679508000 & -1.052441000 & 3.900878000 \\
\hline $\mathrm{H}$ & -3.190974000 & 1.404887000 & 1.859526000 & H & -2.156175000 & -1.603580000 & 4.676130000 \\
\hline H & -5.460417000 & 0.845894000 & 2.769057000 & H & -1.108461000 & -1.315250000 & 2.453458000 \\
\hline C & -3.198964000 & 2.729751000 & -1.023035000 & C & -2.467837000 & -1.497822000 & -0.974518000 \\
\hline H & -2.107015000 & 2.758842000 & -1.093076000 & C & -3.589651000 & -2.266053000 & -0.629307000 \\
\hline H & -3.486476000 & 3.108500000 & -0.037700000 & $\mathrm{H}$ & -4.178207000 & -2.011184000 & 0.246004000 \\
\hline H & -3.601415000 & 3.419591000 & -1.769990000 & H & -4.815625000 & -3.959755000 & -1.134173000 \\
\hline \multirow[t]{8}{*}{$\mathrm{H}$} & -5.728285000 & -0.335717000 & 1.488636000 & C & -3.948327000 & -3.367251000 & -1.408816000 \\
\hline & & & & C & -3.194784000 & -3.707198000 & -2.533537000 \\
\hline & & & & C & -2.075952000 & -2.946124000 & -2.881246000 \\
\hline & & & & $\mathrm{H}$ & -1.486046000 & -3.210457000 & -3.753556000 \\
\hline & & & & c & -1.709390000 & -1.848863000 & -2.103481000 \\
\hline & & & & $\mathrm{H}$ & -0.833716000 & -1.263903000 & -2.372178000 \\
\hline & & & & H & -3.475681000 & -4.565737000 & -3.135893000 \\
\hline & & & & C & -3.935366000 & -0.497981000 & 4.159452000 \\
\hline
\end{tabular}




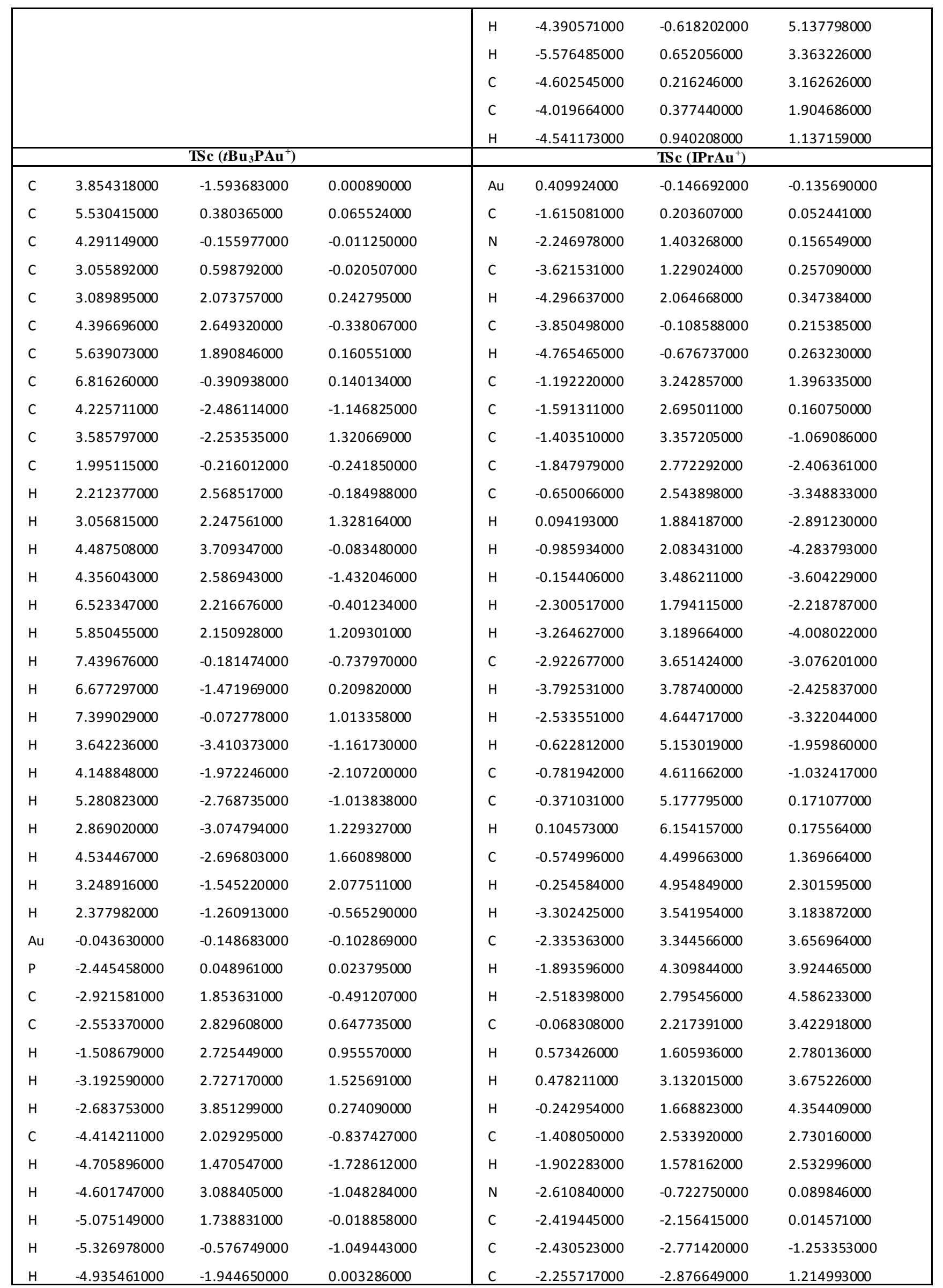




\begin{tabular}{|c|c|c|c|c|c|c|c|}
\hline $\mathrm{H}$ & -5.089648000 & -2.183790000 & -1.731988000 & C & -2.263159000 & -2.215224000 & 2.590206000 \\
\hline c & -4.730022000 & -1.487031000 & -0.966107000 & $\mathrm{H}$ & -2.412284000 & -1.140291000 & 2.452604000 \\
\hline c & -3.225287000 & -1.238428000 & -1.196487000 & $\mathrm{H}$ & -0.091213000 & -1.989215000 & 2.708020000 \\
\hline C & -2.459165000 & -2.577814000 & -1.071521000 & C & -0.911775000 & -2.396230000 & 3.307961000 \\
\hline $\mathrm{H}$ & -2.863302000 & -3.272430000 & -1.816794000 & $\mathrm{H}$ & -0.699488000 & -3.452776000 & 3.502692000 \\
\hline $\mathrm{H}$ & -1.392874000 & -2.450446000 & -1.281501000 & $\mathrm{H}$ & -0.920397000 & -1.877314000 & 4.272089000 \\
\hline $\mathrm{H}$ & -2.560630000 & -3.052561000 & -0.097399000 & $\mathrm{H}$ & -3.447996000 & -2.203420000 & 4.418306000 \\
\hline $\mathrm{H}$ & -0.991631000 & 2.219775000 & -1.480736000 & C & -3.428100000 & -2.729833000 & 3.458624000 \\
\hline $\mathrm{H}$ & -2.298850000 & 3.313034000 & -1.952803000 & $\mathrm{H}$ & -4.393464000 & -2.574371000 & 2.966901000 \\
\hline C & -2.060755000 & 2.271558000 & -1.707931000 & $\mathrm{H}$ & -3.331650000 & -3.799733000 & 3.669732000 \\
\hline $\mathrm{H}$ & -2.246387000 & 1.677255000 & -2.600399000 & $\mathrm{H}$ & -1.960305000 & -4.851391000 & 2.016075000 \\
\hline $\mathrm{H}$ & -3.615876000 & 0.108994000 & -2.909706000 & C & -2.086152000 & -4.263049000 & 1.112454000 \\
\hline $\mathrm{H}$ & -3.315783000 & -1.572621000 & -3.321479000 & $\mathrm{H}$ & -1.959679000 & -5.977164000 & -0.180660000 \\
\hline H & -1.961466000 & -0.540357000 & -2.860060000 & C & -2.085737000 & -4.899811000 & -0.125694000 \\
\hline C & -3.012306000 & -0.761582000 & -2.650262000 & $\mathrm{H}$ & -2.263856000 & -4.669658000 & -2.251717000 \\
\hline $\mathrm{H}$ & -4.551691000 & 1.233428000 & 2.113477000 & C & -2.257415000 & -4.160679000 & -1.292847000 \\
\hline c & -4.435605000 & 0.149303000 & 2.166195000 & $\mathrm{H}$ & -2.731177000 & -0.935968000 & -2.307588000 \\
\hline $\mathrm{H}$ & -4.675223000 & -0.147819000 & 3.193707000 & C & -2.629566000 & -1.997114000 & -2.552954000 \\
\hline H & -5.182780000 & -0.307589000 & 1.514424000 & C & -3.925058000 & -2.427009000 & -3.269737000 \\
\hline C & -2.998567000 & -0.310587000 & 1.845215000 & $\mathrm{H}$ & -3.887408000 & -3.478697000 & -3.571609000 \\
\hline C & -2.009586000 & 0.381824000 & 2.814427000 & $\mathrm{H}$ & -0.496025000 & -1.779447000 & -2.991478000 \\
\hline $\mathrm{H}$ & -2.019136000 & 1.468086000 & 2.747970000 & C & -1.408853000 & -2.129447000 & -3.484277000 \\
\hline $\mathrm{H}$ & -0.982976000 & 0.039223000 & 2.653278000 & $\mathrm{H}$ & -1.249986000 & -3.167190000 & -3.795523000 \\
\hline $\mathrm{H}$ & -2.292408000 & 0.114709000 & 3.839136000 & $\mathrm{H}$ & -1.557468000 & -1.532243000 & -4.389817000 \\
\hline H & -1.888086000 & -2.211381000 & 1.888707000 & $\mathrm{H}$ & -4.076056000 & -1.827800000 & -4.173357000 \\
\hline C & -2.883259000 & -1.824543000 & 2.128197000 & $\mathrm{H}$ & -4.801387000 & -2.297542000 & -2.626769000 \\
\hline $\mathrm{H}$ & -3.630947000 & -2.418143000 & 1.600495000 & $\mathrm{H}$ & 2.639174000 & -1.555521000 & -0.634899000 \\
\hline \multirow[t]{19}{*}{$\mathrm{H}$} & -3.045910000 & -1.983106000 & 3.199993000 & C & 2.407788000 & -0.463724000 & -0.351095000 \\
\hline & & & & C & 3.577193000 & 0.212879000 & -0.202547000 \\
\hline & & & & C & 4.695959000 & -0.701571000 & -0.132703000 \\
\hline & & & & C & 4.062245000 & -2.049014000 & 0.042500000 \\
\hline & & & & C & 3.692880000 & -2.503184000 & 1.423598000 \\
\hline & & & & $\mathrm{H}$ & 3.509914000 & -1.668417000 & 2.099686000 \\
\hline & & & & $\mathrm{H}$ & 2.824750000 & -3.169204000 & 1.412608000 \\
\hline & & & & $\mathrm{H}$ & 4.537826000 & -3.090970000 & 1.814640000 \\
\hline & & & & $\mathrm{H}$ & 3.562966000 & -3.933139000 & -0.900350000 \\
\hline & & & & C & 4.287703000 & -3.119177000 & -0.985624000 \\
\hline & & & & $\mathrm{H}$ & 5.283544000 & -3.549904000 & -0.802389000 \\
\hline & & & & $\mathrm{H}$ & 4.290459000 & -2.719498000 & -2.001773000 \\
\hline & & & & C & 5.999216000 & -0.336975000 & -0.110029000 \\
\hline & & & & C & 7.167090000 & -1.268421000 & 0.035902000 \\
\hline & & & & $\mathrm{H}$ & 7.807862000 & -1.220517000 & -0.853342000 \\
\hline & & & & $\mathrm{H}$ & 7.793836000 & -0.961866000 & 0.882587000 \\
\hline & & & & $\mathrm{H}$ & 6.880410000 & -2.310036000 & 0.194159000 \\
\hline & & & & $\mathrm{H}$ & 7.220945000 & 1.297403000 & -0.751762000 \\
\hline & & & & $\mathrm{H}$ & 6.592908000 & 1.458704000 & 0.869501000 \\
\hline
\end{tabular}




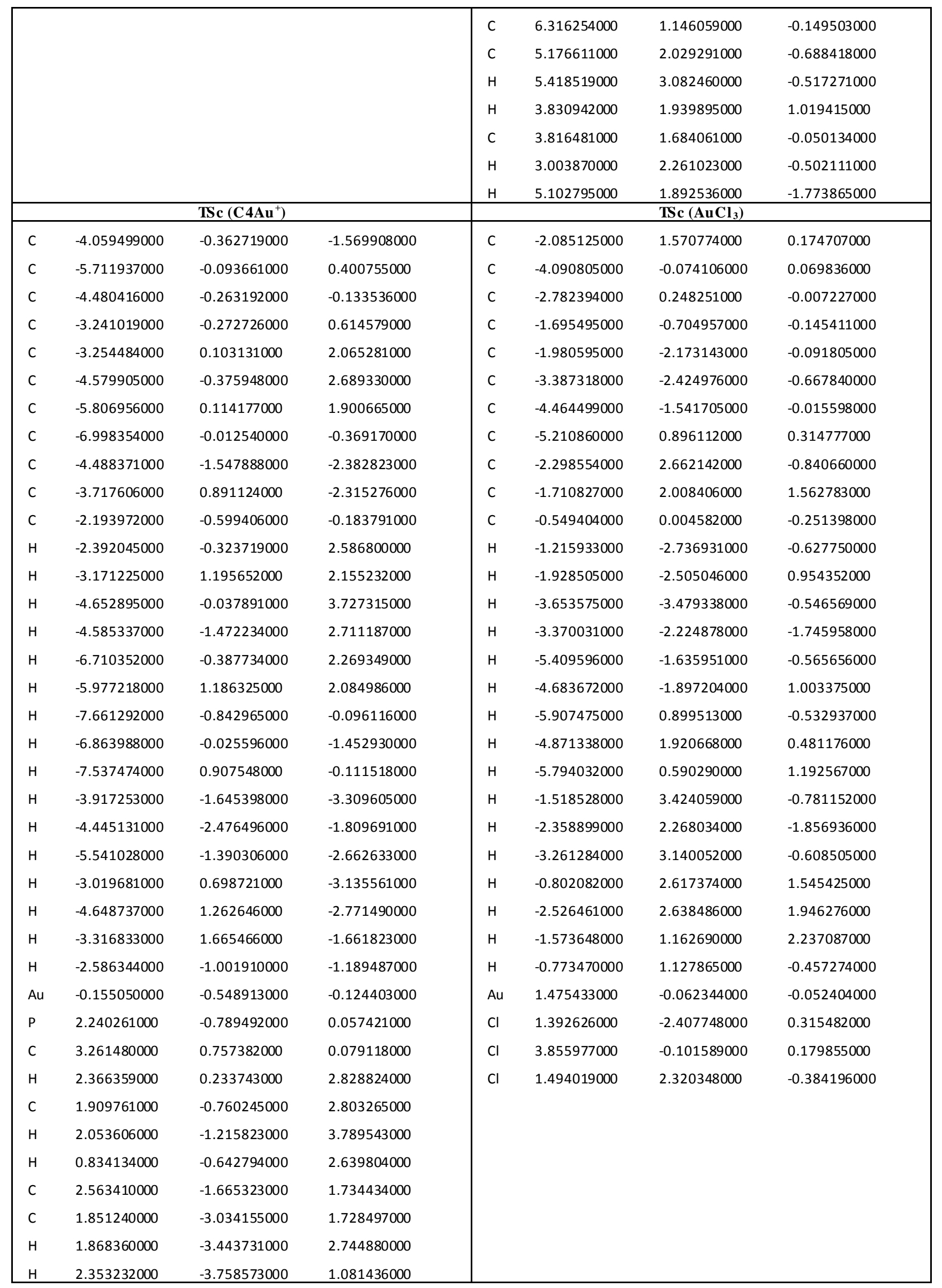




\begin{tabular}{|c|c|c|c|c|c|c|c|}
\hline H & 1.675307000 & -3.619525000 & -0.989044000 & & & & \\
\hline $\mathrm{H}$ & 2.043101000 & -3.407900000 & -2.701574000 & & & & \\
\hline c & 1.750687000 & -2.878912000 & -1.787259000 & & & & \\
\hline $\mathrm{H}$ & 0.804369000 & -2.956618000 & 1.418677000 & & & & \\
\hline $\mathrm{H}$ & 0.756901000 & -2.452627000 & -1.949381000 & & & & \\
\hline $\mathrm{H}$ & 1.840374000 & -0.277871000 & -2.767526000 & & & & \\
\hline C & 2.806846000 & -0.783485000 & -2.664611000 & & & & \\
\hline $\mathrm{H}$ & 3.583176000 & -0.022334000 & -2.556662000 & & & & \\
\hline $\mathrm{H}$ & 2.996751000 & -1.328416000 & -3.596214000 & & & & \\
\hline C & 2.804215000 & -1.788507000 & -1.491796000 & & & & \\
\hline C & 4.183788000 & -2.467228000 & -1.392382000 & & & & \\
\hline $\mathrm{H}$ & 4.383763000 & -2.980856000 & -2.339826000 & & & & \\
\hline $\mathrm{H}$ & 4.220126000 & -3.223971000 & -0.604735000 & & & & \\
\hline H & 4.999779000 & -1.759132000 & -1.239583000 & & & & \\
\hline $\mathrm{H}$ & 4.597179000 & -2.460091000 & 1.387220000 & & & & \\
\hline $\mathrm{H}$ & 4.076212000 & -2.414040000 & 3.068140000 & & & & \\
\hline $\mathrm{H}$ & 4.562033000 & -0.921268000 & 2.271970000 & & & & \\
\hline C & 4.041925000 & -1.868510000 & 2.118119000 & & & & \\
\hline H & 5.105386000 & -0.364847000 & 0.181304000 & & & & \\
\hline C & 4.664762000 & 0.620530000 & 0.129219000 & & & & \\
\hline C & 5.519045000 & 1.718844000 & 0.116590000 & & & & \\
\hline $\mathrm{H}$ & 6.593276000 & 1.567755000 & 0.156326000 & & & & \\
\hline $\mathrm{H}$ & 5.630330000 & 3.873593000 & 0.039998000 & & & & \\
\hline C & 4.982193000 & 3.002708000 & 0.051582000 & & & & \\
\hline C & 3.601170000 & 3.161607000 & 0.001738000 & & & & \\
\hline $\mathrm{H}$ & 3.177220000 & 4.159788000 & -0.046673000 & & & & \\
\hline C & 2.719460000 & 2.064760000 & 0.014538000 & & & & \\
\hline C & 0.679856000 & 2.769022000 & -1.276853000 & & & & \\
\hline C & 1.266585000 & 2.434902000 & -0.046187000 & & & & \\
\hline $\mathrm{H}$ & 1.249808000 & 2.635886000 & -2.191830000 & & & & \\
\hline C & 0.533474000 & 2.651503000 & 1.131313000 & & & & \\
\hline H & 0.987204000 & 2.429212000 & 2.092495000 & & & & \\
\hline C & -0.751857000 & 3.194332000 & 1.078883000 & & & & \\
\hline $\mathrm{H}$ & -1.292781000 & 3.383108000 & 2.001804000 & & & & \\
\hline C & -1.323628000 & 3.529439000 & -0.150561000 & & & & \\
\hline H & -2.309839000 & 3.983802000 & -0.187240000 & & & & \\
\hline C & -0.606833000 & 3.309404000 & -1.328948000 & & & & \\
\hline $\mathrm{H}$ & -1.033882000 & 3.587402000 & -2.288456000 & & & & \\
\hline \multicolumn{4}{|c|}{ Int4 $\left(\mathrm{H}_{3} \mathrm{PAu}^{+}\right)$} & \multicolumn{4}{|c|}{ Int4 $\left(\mathrm{Me}_{3} \mathrm{PAu}^{+}\right)$} \\
\hline C & -2.245512000 & 1.742113000 & 0.021816000 & C & -2.791243000 & 1.757494000 & 0.018262000 \\
\hline $\mathrm{Au}$ & 1.843855000 & -0.066194000 & -0.001005000 & $\mathrm{Au}$ & 1.257924000 & -0.144460000 & -0.018986000 \\
\hline C & -3.781887000 & -0.358134000 & 0.005573000 & C & -4.384195000 & -0.297030000 & 0.019267000 \\
\hline C & -2.562163000 & 0.235365000 & -0.031691000 & C & -3.148442000 & 0.260021000 & -0.028935000 \\
\hline C & -1.369846000 & -0.609667000 & -0.139329000 & C & -1.980046000 & -0.620216000 & -0.143431000 \\
\hline C & -1.500593000 & -2.136687000 & -0.435497000 & C & -2.160420000 & -2.139779000 & -0.434290000 \\
\hline C & -2.688950000 & -2.645101000 & 0.383328000 & $\mathrm{C}$ & -3.356033000 & -2.614282000 & 0.394108000 \\
\hline
\end{tabular}




\begin{tabular}{|c|c|c|c|c|c|c|c|}
\hline c & -3.951842000 & -1.866073000 & 0.024693000 & C & -4.598840000 & -1.799540000 & 0.043519000 \\
\hline c & -5.090325000 & 0.384624000 & 0.045825000 & C & -5.671245000 & 0.482816000 & 0.067383000 \\
\hline c & -2.814745000 & 2.459357000 & 1.257993000 & c & -3.327327000 & 2.492572000 & 1.259132000 \\
\hline C & -2.583189000 & 2.470884000 & -1.292497000 & C & -3.124494000 & 2.493060000 & -1.293579000 \\
\hline c & -0.109037000 & -0.325641000 & -0.071016000 & C & -0.711419000 & -0.364097000 & -0.081309000 \\
\hline $\mathrm{H}$ & -0.584448000 & -2.686835000 & -0.204690000 & H & -1.257870000 & -2.714746000 & -0.210253000 \\
\hline $\mathrm{H}$ & -1.683755000 & -2.226544000 & -1.511834000 & H & -2.356470000 & -2.230657000 & -1.508509000 \\
\hline $\mathrm{H}$ & -2.821866000 & -3.712063000 & 0.173862000 & $\mathrm{H}$ & -3.522694000 & -3.677513000 & 0.190147000 \\
\hline $\mathrm{H}$ & -2.468956000 & -2.549501000 & 1.452112000 & H & -3.125296000 & -2.520944000 & 1.460984000 \\
\hline $\mathrm{H}$ & -4.754624000 & -2.112559000 & 0.729966000 & $\mathrm{H}$ & -5.402934000 & -2.020927000 & 0.755775000 \\
\hline $\mathrm{H}$ & -4.325930000 & -2.181154000 & -0.961043000 & $\mathrm{H}$ & -4.989403000 & -2.106737000 & -0.938359000 \\
\hline $\mathrm{H}$ & -5.014177000 & 1.433761000 & -0.229864000 & $\mathrm{H}$ & -5.566635000 & 1.530331000 & -0.205247000 \\
\hline $\mathrm{H}$ & -5.806354000 & -0.095378000 & -0.631202000 & H & -6.403152000 & 0.026414000 & -0.609226000 \\
\hline H & -5.527046000 & 0.324423000 & 1.051122000 & H & -6.106708000 & 0.431923000 & 1.073743000 \\
\hline $\mathrm{H}$ & -2.410264000 & 3.474984000 & 1.313301000 & H & -2.897165000 & 3.498088000 & 1.306800000 \\
\hline $\mathrm{H}$ & -2.543372000 & 1.936835000 & 2.180327000 & $\mathrm{H}$ & -3.057415000 & 1.965654000 & 2.179488000 \\
\hline $\mathrm{H}$ & -3.903165000 & 2.546467000 & 1.229358000 & $\mathrm{H}$ & -4.413638000 & 2.605891000 & 1.244389000 \\
\hline $\mathrm{H}$ & -2.222773000 & 3.503579000 & -1.252608000 & $\mathrm{H}$ & -2.736381000 & 3.516028000 & -1.260346000 \\
\hline $\mathrm{H}$ & -3.660364000 & 2.507260000 & -1.477248000 & H & -4.202194000 & 2.557241000 & -1.467071000 \\
\hline $\mathrm{H}$ & -2.114325000 & 1.980987000 & -2.151471000 & $\mathrm{H}$ & -2.678043000 & 1.988754000 & -2.156236000 \\
\hline $\mathrm{H}$ & -1.148846000 & 1.796319000 & 0.125145000 & H & -1.692986000 & 1.784476000 & 0.108745000 \\
\hline $\mathrm{H}$ & 4.802425000 & 0.253539000 & 1.359516000 & $\mathrm{P}$ & 3.622114000 & 0.146768000 & 0.051080000 \\
\hline$P$ & 4.203672000 & 0.273132000 & 0.087884000 & C & 4.378031000 & -0.388352000 & 1.635116000 \\
\hline $\mathrm{H}$ & 4.670975000 & 1.491965000 & -0.433986000 & $\mathrm{H}$ & 4.167154000 & -1.447202000 & 1.803812000 \\
\hline \multirow[t]{11}{*}{$\mathrm{H}$} & 4.985738000 & -0.659991000 & -0.614820000 & $\mathrm{H}$ & 5.461084000 & -0.235717000 & 1.613301000 \\
\hline & & & & $\mathrm{H}$ & 3.948899000 & 0.186280000 & 2.459523000 \\
\hline & & & & C & 4.118172000 & 1.899829000 & -0.172178000 \\
\hline & & & & $\mathrm{H}$ & 5.207837000 & 1.993111000 & -0.143451000 \\
\hline & & & & $\mathrm{H}$ & 3.750042000 & 2.266728000 & -1.133303000 \\
\hline & & & & $\mathrm{H}$ & 3.683270000 & 2.509725000 & 0.623367000 \\
\hline & & & & C & 4.500795000 & -0.788419000 & -1.261044000 \\
\hline & & & & H & 4.302703000 & -1.857129000 & -1.148427000 \\
\hline & & & & H & 4.141779000 & -0.469720000 & -2.242587000 \\
\hline & & & & $\mathrm{H}$ & 5.578723000 & -0.612973000 & -1.195487000 \\
\hline & \multicolumn{3}{|c|}{ Int4 $\left(\mathrm{Et}_{3} \mathrm{PAu}^{+}\right)$} & \multicolumn{4}{|c|}{ Int4 $\left(\mathrm{Ph}_{3} \mathrm{PAu}^{+}\right)$} \\
\hline C & 3.291810000 & 1.684432000 & 0.325977000 & C & 4.378524000 & 1.721738000 & -0.026711000 \\
\hline $\mathrm{Au}$ & -0.774659000 & -0.142361000 & 0.053264000 & $\mathrm{Au}$ & 0.332846000 & -0.191161000 & 0.044999000 \\
\hline C & 4.862458000 & -0.326684000 & -0.169077000 & C & 5.973726000 & -0.329925000 & -0.005155000 \\
\hline C & 3.634646000 & 0.211606000 & 0.034705000 & c & 4.737442000 & 0.225623000 & 0.036047000 \\
\hline C & 2.459717000 & -0.667340000 & -0.012233000 & C & 3.569240000 & -0.655095000 & 0.161045000 \\
\hline C & 2.633814000 & -2.212823000 & -0.056335000 & C & 3.752802000 & -2.168668000 & 0.468898000 \\
\hline C & 3.800372000 & -2.506936000 & -1.001307000 & C & 4.948597000 & -2.652823000 & -0.353385000 \\
\hline C & 5.061063000 & -1.789480000 & -0.523638000 & C & 6.190713000 & -1.832442000 & -0.012410000 \\
\hline C & 6.156367000 & 0.438465000 & -0.082332000 & C & 7.260480000 & 0.450518000 & -0.059551000 \\
\hline C & 3.804334000 & 2.672846000 & -0.736092000 & C & 4.913580000 & 2.444779000 & -1.275162000 \\
\hline C & 3.664347000 & 2.112067000 & 1.758291000 & C & 4.710447000 & 2.471049000 & 1.277734000 \\
\hline
\end{tabular}




\begin{tabular}{|c|c|c|c|c|c|c|c|}
\hline c & 1.193594000 & -0.389827000 & 0.010248000 & C & 2.301142000 & -0.392671000 & 0.093921000 \\
\hline $\mathrm{H}$ & 1.719060000 & -2.723890000 & -0.368489000 & $\mathrm{H}$ & 2.850071000 & -2.746191000 & 0.252223000 \\
\hline $\mathrm{H}$ & 2.861078000 & -2.529917000 & 0.967713000 & $\mathrm{H}$ & 3.950116000 & -2.248912000 & 1.543882000 \\
\hline $\mathrm{H}$ & 3.962632000 & -3.589778000 & -1.031943000 & $\mathrm{H}$ & 5.117273000 & -3.713540000 & -0.138309000 \\
\hline $\mathrm{H}$ & 3.540577000 & -2.189388000 & -2.017206000 & $\mathrm{H}$ & 4.717832000 & -2.571090000 & -1.421291000 \\
\hline $\mathrm{H}$ & 5.841112000 & -1.855131000 & -1.291882000 & $\mathrm{H}$ & 6.995120000 & -2.061086000 & -0.722169000 \\
\hline H & 5.478108000 & -2.301914000 & 0.356517000 & $\mathrm{H}$ & 6.581452000 & -2.128599000 & 0.972861000 \\
\hline $\mathrm{H}$ & 6.070163000 & 1.402313000 & 0.414014000 & $\mathrm{H}$ & 7.152918000 & 1.502881000 & 0.192462000 \\
\hline $\mathrm{H}$ & 6.900420000 & -0.157339000 & 0.459130000 & $\mathrm{H}$ & 7.988076000 & 0.008448000 & 0.631281000 \\
\hline $\mathrm{H}$ & 6.565657000 & 0.605476000 & -1.087096000 & $\mathrm{H}$ & 7.703771000 & 0.381257000 & -1.061400000 \\
\hline $\mathrm{H}$ & 3.378451000 & 3.664608000 & -0.552789000 & $\mathrm{H}$ & 4.481748000 & 3.449092000 & -1.333511000 \\
\hline $\mathrm{H}$ & 3.511527000 & 2.359959000 & -1.743039000 & $\mathrm{H}$ & 4.644038000 & 1.907570000 & -2.189635000 \\
\hline H & 4.891288000 & 2.780851000 & -0.721427000 & $\mathrm{H}$ & 5.999783000 & 2.559884000 & -1.262066000 \\
\hline H & 3.286643000 & 3.119391000 & 1.960476000 & $\mathrm{H}$ & 4.321927000 & 3.493561000 & 1.233993000 \\
\hline $\mathrm{H}$ & 4.746625000 & 2.130662000 & 1.913514000 & $\mathrm{H}$ & 5.788025000 & 2.537031000 & 1.451890000 \\
\hline $\mathrm{H}$ & 3.233373000 & 1.432946000 & 2.500498000 & $\mathrm{H}$ & 4.262686000 & 1.975457000 & 2.144713000 \\
\hline $\mathrm{H}$ & 2.191988000 & 1.735469000 & 0.272738000 & $\mathrm{H}$ & 3.280576000 & 1.747183000 & -0.117629000 \\
\hline$P$ & -3.138814000 & 0.169754000 & 0.115460000 & $\mathrm{P}$ & -2.046818000 & 0.023129000 & -0.005043000 \\
\hline C & -3.610650000 & 1.953017000 & 0.251475000 & C & -2.815561000 & -1.479164000 & -0.710711000 \\
\hline $\mathrm{H}$ & -3.213299000 & 2.300740000 & 1.211381000 & C & -2.227520000 & -2.070101000 & -1.841864000 \\
\hline $\mathrm{H}$ & -4.703054000 & 2.004645000 & 0.321760000 & $\mathrm{H}$ & -1.325415000 & -1.644160000 & -2.273555000 \\
\hline C & -3.091694000 & 2.828446000 & -0.896693000 & $\mathrm{H}$ & -2.344960000 & -3.652454000 & -3.293052000 \\
\hline $\mathrm{H}$ & -3.505503000 & 2.523864000 & -1.862228000 & C & -2.802321000 & -3.201392000 & -2.417930000 \\
\hline $\mathrm{H}$ & -2.000024000 & 2.791859000 & -0.968341000 & C & -3.959869000 & -3.756029000 & -1.865219000 \\
\hline $\mathrm{H}$ & -3.380528000 & 3.869569000 & -0.729418000 & $\mathrm{H}$ & -4.402844000 & -4.641299000 & -2.310966000 \\
\hline C & -3.926804000 & -0.653001000 & 1.571454000 & $\mathrm{H}$ & -5.442101000 & -3.606526000 & -0.306664000 \\
\hline $\mathrm{H}$ & -3.516806000 & -0.156514000 & 2.457839000 & C & -4.544346000 & -3.175425000 & -0.738681000 \\
\hline $\mathrm{H}$ & -4.999093000 & -0.428208000 & 1.546770000 & C & -3.977297000 & -2.038623000 & -0.159496000 \\
\hline $\mathrm{H}$ & -4.118425000 & -2.691903000 & 0.787050000 & $\mathrm{H}$ & -4.435930000 & -1.594629000 & 0.717810000 \\
\hline C & -3.682884000 & -2.166164000 & 1.641805000 & C & -2.571822000 & 1.439633000 & -1.034556000 \\
\hline H & -2.613347000 & -2.397336000 & 1.675163000 & C & -3.687330000 & 1.357558000 & -1.881007000 \\
\hline H & -3.658800000 & -1.557051000 & -1.467460000 & $\mathrm{H}$ & -4.248219000 & 0.431982000 & -1.960324000 \\
\hline C & -3.946684000 & -0.501805000 & -1.408966000 & C & -4.074536000 & 2.469381000 & -2.631249000 \\
\hline $\mathrm{H}$ & -3.455305000 & -0.009350000 & -2.255044000 & $\mathrm{H}$ & -4.937102000 & 2.400283000 & -3.286752000 \\
\hline $\mathrm{H}$ & -4.140596000 & -2.575022000 & 2.546577000 & C & -3.355524000 & 3.662367000 & -2.542035000 \\
\hline $\mathrm{H}$ & -5.980062000 & -0.862811000 & -0.670238000 & $\mathrm{H}$ & -3.658358000 & 4.523485000 & -3.129699000 \\
\hline C & -5.470760000 & -0.342597000 & -1.486457000 & $\mathrm{H}$ & -1.680610000 & 4.673961000 & -1.632320000 \\
\hline H & -5.836501000 & -0.769574000 & -2.424528000 & C & -2.243113000 & 3.748043000 & -1.700764000 \\
\hline \multirow[t]{8}{*}{$\mathrm{H}$} & -5.775348000 & 0.707626000 & -1.464685000 & C & -1.848546000 & 2.641056000 & -0.951655000 \\
\hline & & & & $\mathrm{H}$ & -0.980351000 & 2.712263000 & -0.301280000 \\
\hline & & & & C & -2.750322000 & 0.273695000 & 1.661978000 \\
\hline & & & & C & -2.149046000 & -0.362139000 & 2.760280000 \\
\hline & & & & $\mathrm{H}$ & -1.260525000 & -0.971797000 & 2.618234000 \\
\hline & & & & $\mathrm{H}$ & -2.218729000 & -0.706019000 & 4.882184000 \\
\hline & & & & C & -2.688535000 & -0.212727000 & 4.037008000 \\
\hline & & & & C & -3.824910000 & 0.577139000 & 4.227445000 \\
\hline
\end{tabular}




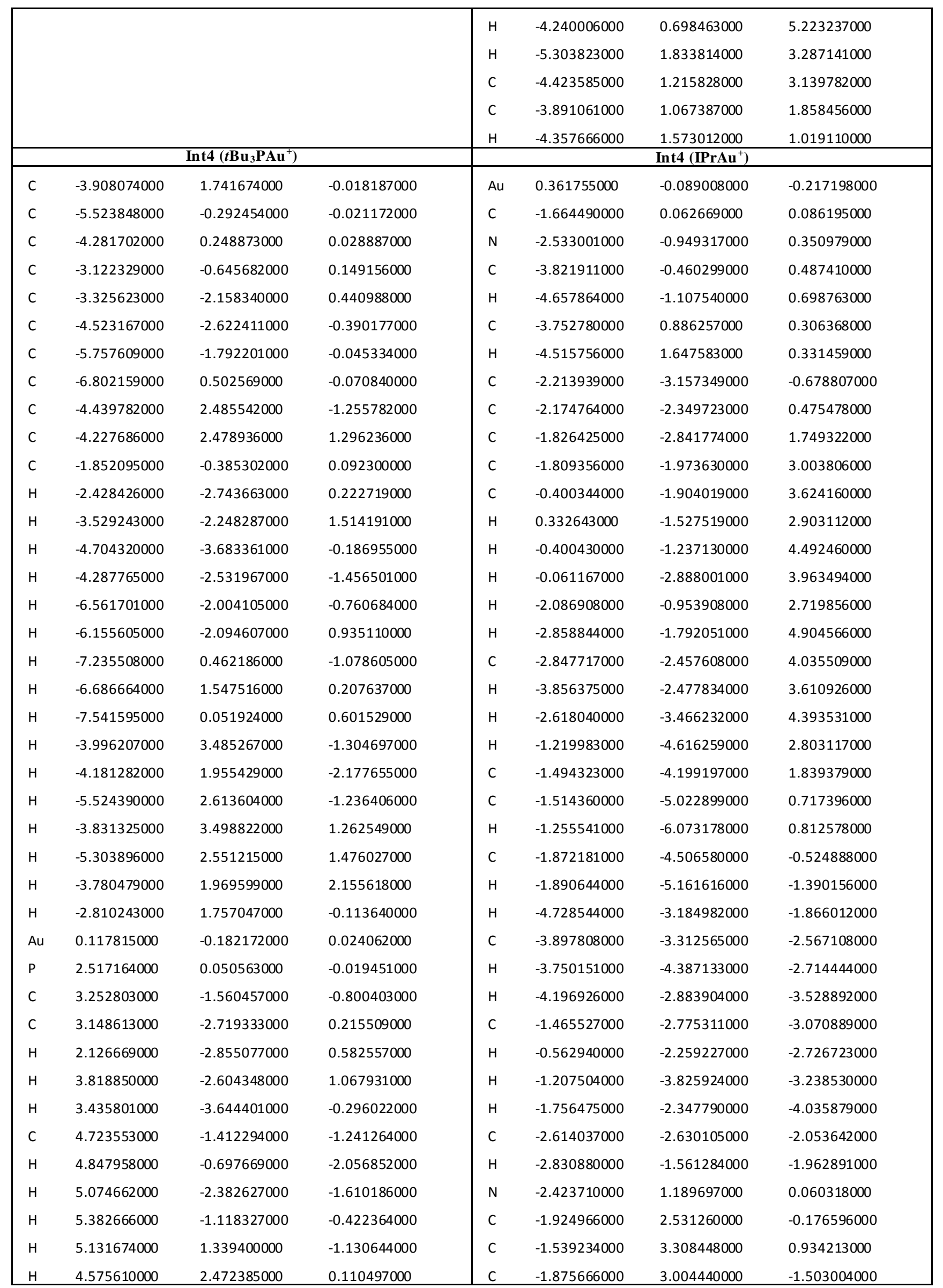




\begin{tabular}{|c|c|c|c|c|c|c|c|}
\hline $\mathrm{H}$ & 4.559231000 & 2.942834000 & -1.583753000 & C & -2.322441000 & 2.174836000 & -2.703213000 \\
\hline c & 4.387685000 & 2.104163000 & -0.899659000 & $\mathrm{H}$ & -2.635075000 & 1.189969000 & -2.342990000 \\
\hline c & 2.940797000 & 1.605279000 & -1.091543000 & $\mathrm{H}$ & -0.314601000 & 1.461679000 & -3.212116000 \\
\hline C & 1.950171000 & 2.743820000 & -0.746679000 & C & -1.167482000 & 1.947179000 & -3.697811000 \\
\hline $\mathrm{H}$ & 2.166631000 & 3.593277000 & -1.404187000 & $\mathrm{H}$ & -0.815376000 & 2.889804000 & -4.128980000 \\
\hline $\mathrm{H}$ & 0.913072000 & 2.445105000 & -0.930689000 & $\mathrm{H}$ & -1.499483000 & 1.309941000 & -4.523855000 \\
\hline $\mathrm{H}$ & 2.026765000 & 3.100118000 & 0.278744000 & $\mathrm{H}$ & -3.878288000 & 2.180516000 & -4.228995000 \\
\hline $\mathrm{H}$ & 1.347287000 & -2.149908000 & -1.731493000 & C & -3.539338000 & 2.813022000 & -3.402314000 \\
\hline $\mathrm{H}$ & 2.786036000 & -2.919043000 & -2.407729000 & $\mathrm{H}$ & -4.377012000 & 2.944283000 & -2.710136000 \\
\hline C & 2.389543000 & -1.975368000 & -2.016474000 & $\mathrm{H}$ & -3.293762000 & 3.796337000 & -3.815850000 \\
\hline $\mathrm{H}$ & 2.402534000 & -1.255594000 & -2.832626000 & $\mathrm{H}$ & -1.346443000 & 4.708052000 & -2.704910000 \\
\hline $\mathrm{H}$ & 3.446811000 & 0.576502000 & -2.985858000 & C & -1.400735000 & 4.307293000 & -1.697651000 \\
\hline $\mathrm{H}$ & 2.819441000 & 2.209423000 & -3.152278000 & $\mathrm{H}$ & -0.641896000 & 6.107364000 & -0.799453000 \\
\hline H & 1.709466000 & 0.892563000 & -2.774309000 & C & -1.003141000 & 5.098552000 & -0.623632000 \\
\hline C & 2.715413000 & 1.278650000 & -2.584175000 & $\mathrm{H}$ & -0.769502000 & 5.234522000 & 1.504645000 \\
\hline $\mathrm{H}$ & 4.937818000 & -0.968053000 & 1.801692000 & C & -1.074546000 & 4.603906000 & 0.675637000 \\
\hline c & 4.630908000 & 0.062348000 & 1.991290000 & $\mathrm{H}$ & -1.998476000 & 1.780322000 & 2.359643000 \\
\hline $\mathrm{H}$ & 4.889127000 & 0.284477000 & 3.032849000 & C & -1.619671000 & 2.806795000 & 2.373560000 \\
\hline H & 5.231609000 & 0.720841000 & 1.361318000 & C & -2.609835000 & 3.645802000 & 3.205891000 \\
\hline C & 3.114294000 & 0.277161000 & 1.807238000 & $\mathrm{H}$ & -2.280226000 & 4.685981000 & 3.292855000 \\
\hline C & 2.339926000 & -0.704569000 & 2.719879000 & $\mathrm{H}$ & 0.463510000 & 2.136735000 & 2.474424000 \\
\hline $\mathrm{H}$ & 2.532077000 & -1.753213000 & 2.502370000 & C & -0.230344000 & 2.768220000 & 3.039094000 \\
\hline $\mathrm{H}$ & 1.259867000 & -0.535524000 & 2.669786000 & $\mathrm{H}$ & 0.208916000 & 3.768301000 & 3.110481000 \\
\hline $\mathrm{H}$ & 2.652466000 & -0.523826000 & 3.754457000 & $\mathrm{H}$ & -0.307053000 & 2.367048000 & 4.054880000 \\
\hline H & 1.681765000 & 1.913194000 & 2.162158000 & $\mathrm{H}$ & -2.694830000 & 3.239080000 & 4.218623000 \\
\hline C & 2.745636000 & 1.695258000 & 2.296185000 & $\mathrm{H}$ & -3.608149000 & 3.650634000 & 2.757193000 \\
\hline $\mathrm{H}$ & 3.332196000 & 2.481213000 & 1.819063000 & $\mathrm{H}$ & 3.310219000 & 0.930284000 & 1.245317000 \\
\hline \multirow[t]{19}{*}{$\mathrm{H}$} & 2.954099000 & 1.747668000 & 3.370298000 & C & 2.295963000 & -0.245158000 & -0.499866000 \\
\hline & & & & C & 3.554173000 & -0.422316000 & -0.770393000 \\
\hline & & & & C & 4.738092000 & 0.010581000 & -0.010788000 \\
\hline & & & & C & 4.409255000 & 0.942110000 & 1.169778000 \\
\hline & & & & C & 4.795781000 & 2.408374000 & 0.897624000 \\
\hline & & & & $\mathrm{H}$ & 4.360827000 & 2.764642000 & -0.041397000 \\
\hline & & & & $\mathrm{H}$ & 4.431057000 & 3.051213000 & 1.705334000 \\
\hline & & & & $\mathrm{H}$ & 5.879250000 & 2.543013000 & 0.834596000 \\
\hline & & & & $\mathrm{H}$ & 4.504542000 & 1.057326000 & 3.333773000 \\
\hline & & & & C & 4.922087000 & 0.440587000 & 2.531181000 \\
\hline & & & & $\mathrm{H}$ & 6.009950000 & 0.491674000 & 2.616653000 \\
\hline & & & & $\mathrm{H}$ & 4.620321000 & -0.596124000 & 2.709746000 \\
\hline & & & & C & 5.961858000 & -0.421632000 & -0.401185000 \\
\hline & & & & C & 7.261869000 & -0.004603000 & 0.235712000 \\
\hline & & & & $\mathrm{H}$ & 7.677664000 & -0.830681000 & 0.827208000 \\
\hline & & & & $\mathrm{H}$ & 7.999447000 & 0.217813000 & -0.544611000 \\
\hline & & & & $\mathrm{H}$ & 7.182020000 & 0.867746000 & 0.880282000 \\
\hline & & & & $\mathrm{H}$ & 6.929973000 & -2.121781000 & -1.255295000 \\
\hline & & & & $\mathrm{H}$ & 6.567544000 & -0.856711000 & -2.403021000 \\
\hline
\end{tabular}




\begin{tabular}{|c|c|c|c|c|c|c|c|}
\hline & & & & C & 6.152060000 & -1.403959000 & -1.543151000 \\
\hline & & & & C & 4.884734000 & -2.148824000 & -1.958136000 \\
\hline & & & & $\mathrm{H}$ & 5.041311000 & -2.674274000 & -2.906353000 \\
\hline & & & & $\mathrm{H}$ & 3.953802000 & -0.406479000 & -2.875690000 \\
\hline & & & & C & 3.723637000 & -1.166184000 & -2.119590000 \\
\hline & & & & $\mathrm{H}$ & 2.803390000 & -1.671004000 & -2.425083000 \\
\hline & & & & $\mathrm{H}$ & 4.624177000 & -2.903946000 & -1.208001000 \\
\hline & & Int4 $\left(\mathrm{C}^{2} \mathrm{Au}^{+}\right)$ & & & & Int4 $\left(\mathrm{AuCl}_{3}\right)$ & \\
\hline C & -4.068863000 & -1.860082000 & -0.385514000 & C & -2.686352000 & -1.649571000 & 0.236375000 \\
\hline $\mathrm{Au}$ & -0.149879000 & -0.058902000 & 0.157356000 & C & -4.005875000 & 0.559503000 & -0.097494000 \\
\hline C & -5.750561000 & 0.095182000 & -0.098750000 & C & -2.850539000 & -0.130280000 & 0.068899000 \\
\hline C & -4.492793000 & -0.410928000 & -0.087109000 & C & -1.589576000 & 0.626909000 & 0.111239000 \\
\hline c & -3.371244000 & 0.483290000 & 0.242617000 & C & -1.619295000 & 2.183059000 & 0.274241000 \\
\hline C & -3.644510000 & 1.908151000 & 0.787979000 & C & -2.703958000 & 2.694460000 & -0.672002000 \\
\hline C & -4.824726000 & 2.482490000 & 0.003418000 & C & -4.049023000 & 2.059280000 & -0.327704000 \\
\hline C & -6.039928000 & 1.567026000 & 0.136278000 & C & -5.376530000 & -0.067044000 & -0.088486000 \\
\hline c & -6.997283000 & -0.712428000 & -0.349900000 & C & -3.304390000 & -2.479849000 & -0.903311000 \\
\hline C & -4.511626000 & -2.370933000 & -1.768153000 & C & -3.124172000 & -2.140031000 & 1.630404000 \\
\hline C & -4.432785000 & -2.837190000 & 0.748899000 & C & -0.342111000 & 0.299909000 & 0.072297000 \\
\hline C & -2.091162000 & 0.266580000 & 0.184724000 & H & -0.646605000 & 2.638966000 & 0.079362000 \\
\hline $\mathrm{H}$ & -2.758467000 & 2.543950000 & 0.719401000 & $\mathrm{H}$ & -1.872169000 & 2.370467000 & 1.323880000 \\
\hline $\mathrm{H}$ & -3.896417000 & 1.796517000 & 1.849283000 & H & -2.759613000 & 3.785294000 & -0.585415000 \\
\hline $\mathrm{H}$ & -5.055036000 & 3.482835000 & 0.385866000 & $\mathrm{H}$ & -2.423454000 & 2.467039000 & -1.706386000 \\
\hline $\mathrm{H}$ & -4.545810000 & 2.594307000 & -1.050500000 & $\mathrm{H}$ & -4.776497000 & 2.264308000 & -1.123609000 \\
\hline $\mathrm{H}$ & -6.823355000 & 1.880953000 & -0.564451000 & $\mathrm{H}$ & -4.468256000 & 2.530435000 & 0.574587000 \\
\hline $\mathrm{H}$ & -6.486825000 & 1.674612000 & 1.136382000 & $\mathrm{H}$ & -5.776552000 & -0.124423000 & -1.109311000 \\
\hline $\mathrm{H}$ & -6.853460000 & -1.788051000 & -0.278439000 & $\mathrm{H}$ & -5.401556000 & -1.065152000 & 0.342023000 \\
\hline $\mathrm{H}$ & -7.772408000 & -0.425858000 & 0.370758000 & $\mathrm{H}$ & -6.067474000 & 0.566340000 & 0.480625000 \\
\hline $\mathrm{H}$ & -7.402128000 & -0.486342000 & -1.344923000 & $\mathrm{H}$ & -2.980354000 & -3.521267000 & -0.809413000 \\
\hline $\mathrm{H}$ & -4.036339000 & -3.335148000 & -1.976560000 & H & -2.971805000 & -2.115064000 & -1.879866000 \\
\hline $\mathrm{H}$ & -4.220578000 & -1.672430000 & -2.558863000 & $\mathrm{H}$ & -4.397347000 & -2.475762000 & -0.895067000 \\
\hline $\mathrm{H}$ & -5.591430000 & -2.521472000 & -1.836204000 & H & -2.867050000 & -3.197329000 & 1.750160000 \\
\hline $\mathrm{H}$ & -3.998913000 & -3.822828000 & 0.551140000 & $\mathrm{H}$ & -4.202317000 & -2.041378000 & 1.790148000 \\
\hline $\mathrm{H}$ & -5.513529000 & -2.969769000 & 0.849181000 & $\mathrm{H}$ & -2.616236000 & -1.580889000 & 2.422439000 \\
\hline $\mathrm{H}$ & -4.050774000 & -2.485307000 & 1.712374000 & $\mathrm{H}$ & -1.608578000 & -1.837371000 & 0.178409000 \\
\hline $\mathrm{H}$ & -2.968571000 & -1.835501000 & -0.417773000 & $\mathrm{Au}$ & 1.604062000 & -0.020840000 & -0.004808000 \\
\hline$P$ & 2.089325000 & -0.959678000 & 0.086071000 & $\mathrm{Cl}$ & 2.052564000 & 2.299840000 & 0.357035000 \\
\hline C & 2.142963000 & -2.152153000 & -1.426685000 & $\mathrm{Cl}$ & 3.909318000 & -0.491393000 & -0.126068000 \\
\hline $\mathrm{H}$ & 1.488632000 & -0.492939000 & -2.715451000 & $\mathrm{Cl}$ & 1.039642000 & -2.323500000 & -0.365054000 \\
\hline C & 2.273910000 & -1.255803000 & -2.677161000 & & & & \\
\hline $\mathrm{H}$ & 2.168735000 & -1.881948000 & -3.570008000 & & & & \\
\hline C & 0.799993000 & -2.911331000 & -1.512244000 & & & & \\
\hline $\mathrm{H}$ & 0.605769000 & -3.532770000 & -0.636103000 & & & & \\
\hline H & 0.830692000 & -3.572556000 & -2.385431000 & & & & \\
\hline $\mathrm{H}$ & -0.044877000 & -2.229443000 & -1.643451000 & & & & \\
\hline $\mathrm{H}$ & 0.361980000 & -2.629043000 & 1.778088000 & & & & \\
\hline
\end{tabular}




\begin{tabular}{|c|c|c|c|c|c|c|c|}
\hline C & 1.392581000 & -2.952093000 & 1.957122000 & & & & \\
\hline $\mathrm{H}$ & 1.611052000 & -3.799953000 & 1.302856000 & & & & \\
\hline $\mathrm{H}$ & 1.450757000 & -3.315873000 & 2.988885000 & & & & \\
\hline $\mathrm{H}$ & 3.200812000 & -3.885600000 & -0.571231000 & & & & \\
\hline C & 3.285678000 & -3.185067000 & -1.405847000 & & & & \\
\hline $\mathrm{H}$ & 3.232254000 & -3.775906000 & -2.327295000 & & & & \\
\hline $\mathrm{H}$ & 4.276045000 & -2.727143000 & -1.382230000 & & & & \\
\hline $\mathrm{H}$ & 4.127454000 & -3.076441000 & 1.243397000 & & & & \\
\hline C & 3.833427000 & -2.336205000 & 1.989712000 & & & & \\
\hline H & 3.875040000 & -2.827873000 & 2.968067000 & & & & \\
\hline $\mathrm{H}$ & 4.573782000 & -1.533868000 & 2.000165000 & & & & \\
\hline C & 2.403928000 & -1.801342000 & 1.779650000 & & & & \\
\hline C & 2.133304000 & -0.719443000 & 2.848766000 & & & & \\
\hline H & 2.296751000 & -1.155113000 & 3.840722000 & & & & \\
\hline $\mathrm{H}$ & 2.813811000 & 0.131309000 & 2.747684000 & & & & \\
\hline H & 1.103619000 & -0.350560000 & 2.812666000 & & & & \\
\hline C & 3.432803000 & 0.283020000 & -0.168753000 & & & & \\
\hline C & 4.755802000 & -0.188166000 & -0.300580000 & & & & \\
\hline H & 4.964440000 & -1.244255000 & -0.199010000 & & & & \\
\hline C & 5.820934000 & 0.667311000 & -0.565162000 & & & & \\
\hline H & 6.825046000 & 0.266039000 & -0.659233000 & & & & \\
\hline $\mathrm{H}$ & 6.400505000 & 2.715918000 & -0.920830000 & & & & \\
\hline C & 5.583457000 & 2.032565000 & -0.711267000 & & & & \\
\hline C & 4.286196000 & 2.518462000 & -0.582639000 & & & & \\
\hline $\mathrm{H}$ & 4.098704000 & 3.582548000 & -0.686386000 & & & & \\
\hline C & 3.195512000 & 1.673321000 & -0.307623000 & & & & \\
\hline C & 1.874310000 & 2.370053000 & -0.181705000 & & & & \\
\hline C & 1.247763000 & 2.892010000 & -1.326535000 & & & & \\
\hline H & 1.662781000 & 2.674506000 & -2.306265000 & & & & \\
\hline C & 0.123718000 & 3.711739000 & -1.212604000 & & & & \\
\hline H & -0.337022000 & 4.119271000 & -2.107512000 & & & & \\
\hline $\mathrm{H}$ & -1.236657000 & 4.702790000 & 0.136674000 & & & & \\
\hline C & -0.386237000 & 4.032880000 & 0.047947000 & & & & \\
\hline $\mathrm{H}$ & -0.158750000 & 3.770894000 & 2.178124000 & & & & \\
\hline C & 0.218943000 & 3.511355000 & 1.193397000 & & & & \\
\hline C & 1.340594000 & 2.684185000 & 1.080667000 & & & & \\
\hline $\mathrm{H}$ & 1.835405000 & 2.322707000 & 1.976560000 & & & & \\
\hline $\mathrm{H}$ & 3.243276000 & -0.755511000 & -2.729959000 & & & & \\
\hline \multicolumn{4}{|c|}{ TSd $\left(\mathrm{H}_{3} \mathrm{PAu}^{+}\right)$} & \multicolumn{4}{|c|}{$\mathbf{T S d}\left(\mathrm{Me}_{3} \mathbf{P A u}^{+}\right)$} \\
\hline C & -2.496281000 & 1.769877000 & -0.321136000 & C & 3.095788000 & 1.779179000 & 0.318866000 \\
\hline $\mathrm{Au}$ & 1.819442000 & -0.013920000 & -0.017270000 & $\mathrm{Au}$ & -1.238404000 & -0.036337000 & 0.037088000 \\
\hline C & -3.826942000 & -0.475770000 & 0.006713000 & C & 4.428477000 & -0.460256000 & -0.002277000 \\
\hline C & -2.687504000 & 0.254080000 & -0.129476000 & C & 3.284221000 & 0.262653000 & 0.128695000 \\
\hline C & -1.407456000 & -0.479351000 & -0.184886000 & C & 2.007470000 & -0.478708000 & 0.184732000 \\
\hline C & -1.412621000 & -2.009286000 & -0.478158000 & C & 2.025144000 & -2.010577000 & 0.457616000 \\
\hline $\mathrm{C}$ & -2.451013000 & -2.616458000 & 0.462985000 & C & 3.067464000 & -2.601628000 & -0.489177000 \\
\hline
\end{tabular}




\begin{tabular}{|c|c|c|c|c|c|c|c|}
\hline c & -3.814795000 & -1.980900000 & 0.212414000 & C & 4.426705000 & -1.963747000 & -0.221212000 \\
\hline c & -5.238817000 & 0.069919000 & -0.047014000 & C & 5.837769000 & 0.090792000 & 0.070179000 \\
\hline c & -1.353056000 & 2.284573000 & 0.564506000 & c & 1.915027000 & 2.288104000 & -0.520383000 \\
\hline C & -3.714771000 & 2.684115000 & -0.100103000 & C & 4.301319000 & 2.692350000 & 0.028579000 \\
\hline c & -0.161969000 & -0.135541000 & -0.079716000 & C & 0.757424000 & -0.142378000 & 0.095253000 \\
\hline $\mathrm{H}$ & -0.429148000 & -2.467113000 & -0.342361000 & $\mathrm{H}$ & 1.043753000 & -2.471371000 & 0.316731000 \\
\hline $\mathrm{H}$ & -1.692980000 & -2.124324000 & -1.531169000 & H & 2.307694000 & -2.140898000 & 1.508450000 \\
\hline $\mathrm{H}$ & -2.495786000 & -3.698168000 & 0.297338000 & $\mathrm{H}$ & 3.116909000 & -3.685742000 & -0.341586000 \\
\hline $\mathrm{H}$ & -2.136297000 & -2.461419000 & 1.500773000 & H & 2.756806000 & -2.430041000 & -1.525736000 \\
\hline $\mathrm{H}$ & -4.491017000 & -2.209420000 & 1.045516000 & $\mathrm{H}$ & 5.111914000 & -2.181055000 & -1.050270000 \\
\hline $\mathrm{H}$ & -4.288915000 & -2.434495000 & -0.671158000 & $\mathrm{H}$ & 4.893931000 & -2.424784000 & 0.662201000 \\
\hline $\mathrm{H}$ & -5.451130000 & 0.756316000 & 0.776238000 & $\mathrm{H}$ & 6.072858000 & 0.743724000 & -0.774542000 \\
\hline $\mathrm{H}$ & -5.437469000 & 0.600613000 & -0.980941000 & H & 6.011597000 & 0.658156000 & 0.987030000 \\
\hline H & -5.957361000 & -0.749898000 & 0.021290000 & H & 6.558280000 & -0.730250000 & 0.053104000 \\
\hline $\mathrm{H}$ & -1.122668000 & 3.328887000 & 0.335484000 & H & 1.699539000 & 3.335634000 & -0.291007000 \\
\hline $\mathrm{H}$ & -0.404284000 & 1.737803000 & 0.389767000 & $\mathrm{H}$ & 0.973885000 & 1.747500000 & -0.298989000 \\
\hline $\mathrm{H}$ & -1.586258000 & 2.194097000 & 1.629878000 & $\mathrm{H}$ & 2.103895000 & 2.189978000 & -1.593915000 \\
\hline $\mathrm{H}$ & -3.403259000 & 3.722376000 & -0.244883000 & $\mathrm{H}$ & 3.990079000 & 3.733797000 & 0.149585000 \\
\hline $\mathrm{H}$ & -4.108087000 & 2.600105000 & 0.916989000 & H & 4.659637000 & 2.575353000 & -0.998416000 \\
\hline $\mathrm{H}$ & -4.521327000 & 2.491948000 & -0.806521000 & $\mathrm{H}$ & 5.133749000 & 2.528167000 & 0.711148000 \\
\hline $\mathrm{H}$ & -2.174969000 & 1.902585000 & -1.365767000 & H & 2.821409000 & 1.922453000 & 1.375590000 \\
\hline $\mathrm{H}$ & 4.823569000 & -0.527234000 & 1.145397000 & $\mathrm{P}$ & -3.611085000 & 0.045982000 & -0.031889000 \\
\hline$P$ & 4.193707000 & 0.090061000 & 0.050596000 & C & -4.350804000 & -1.334971000 & -0.987909000 \\
\hline $\mathrm{H}$ & 4.751624000 & 1.380001000 & 0.088421000 & $\mathrm{H}$ & -4.071216000 & -2.288785000 & -0.533926000 \\
\hline \multirow[t]{11}{*}{$\mathrm{H}$} & 4.875576000 & -0.485422000 & -1.035692000 & $\mathrm{H}$ & -5.441284000 & -1.248328000 & -1.001871000 \\
\hline & & & & $\mathrm{H}$ & -3.973999000 & -1.314598000 & -2.013365000 \\
\hline & & & & C & -4.240132000 & 1.582356000 & -0.815529000 \\
\hline & & & & $\mathrm{H}$ & -5.334072000 & 1.581502000 & -0.831218000 \\
\hline & & & & $\mathrm{H}$ & -3.888568000 & 2.452106000 & -0.255217000 \\
\hline & & & & $\mathrm{H}$ & -3.864777000 & 1.652846000 & -1.839316000 \\
\hline & & & & C & -4.395132000 & -0.022244000 & 1.625782000 \\
\hline & & & & H & -4.117331000 & -0.953250000 & 2.125844000 \\
\hline & & & & H & -4.044658000 & 0.815537000 & 2.233453000 \\
\hline & & & & $\mathrm{H}$ & -5.484260000 & 0.027658000 & 1.535299000 \\
\hline & \multicolumn{3}{|c|}{$\mathbf{T S d}\left(\mathrm{Et}_{3} \mathbf{P A u}^{+}\right)$} & \multicolumn{4}{|c|}{$\operatorname{TSd}\left(\mathrm{Ph}_{3} \mathrm{PAu}^{+}\right)$} \\
\hline C & -3.624029000 & 1.696466000 & -0.488642000 & C & 4.683862000 & -1.775662000 & -0.295340000 \\
\hline $\mathrm{Au}$ & 0.758114000 & -0.011915000 & -0.093506000 & $\mathrm{Au}$ & 0.346422000 & 0.052905000 & -0.034624000 \\
\hline C & -4.903248000 & -0.506216000 & 0.141038000 & C & 6.014240000 & 0.468223000 & -0.020295000 \\
\hline C & -3.775348000 & 0.213526000 & -0.101399000 & c & 4.868202000 & -0.255360000 & -0.129234000 \\
\hline C & -2.485342000 & -0.506477000 & -0.084337000 & C & 3.591302000 & 0.487132000 & -0.185772000 \\
\hline C & -2.478578000 & -2.060963000 & -0.146052000 & C & 3.610043000 & 2.016632000 & -0.468867000 \\
\hline C & -3.493509000 & -2.536499000 & 0.891101000 & C & 4.660654000 & 2.613893000 & 0.464757000 \\
\hline C & -4.868691000 & -1.966066000 & 0.560864000 & C & 6.016580000 & 1.973990000 & 0.185135000 \\
\hline C & -6.324277000 & 0.003060000 & 0.015283000 & C & 7.422637000 & -0.083764000 & -0.106727000 \\
\hline C & -2.423791000 & 2.326503000 & 0.232763000 & C & 3.491897000 & -2.273309000 & 0.535466000 \\
\hline C & -4.836595000 & 2.619400000 & -0.263388000 & C & 5.886769000 & -2.679580000 & 0.034498000 \\
\hline
\end{tabular}




\begin{tabular}{|c|c|c|c|c|c|c|c|}
\hline c & -1.239931000 & -0.140378000 & -0.072516000 & C & 2.340367000 & 0.153563000 & -0.090541000 \\
\hline $\mathrm{H}$ & -1.485886000 & -2.481005000 & 0.036851000 & $\mathrm{H}$ & 2.629793000 & 2.478610000 & -0.323910000 \\
\hline $\mathrm{H}$ & -2.776436000 & -2.338831000 & -1.163518000 & $\mathrm{H}$ & 3.884721000 & 2.140952000 & -1.522732000 \\
\hline $\mathrm{H}$ & -3.523236000 & -3.631324000 & 0.894290000 & $\mathrm{H}$ & 4.709112000 & 3.697524000 & 0.311936000 \\
\hline $\mathrm{H}$ & -3.171094000 & -2.218831000 & 1.888959000 & $\mathrm{H}$ & 4.360626000 & 2.446550000 & 1.505338000 \\
\hline $\mathrm{H}$ & -5.539211000 & -2.080812000 & 1.421998000 & $\mathrm{H}$ & 6.712846000 & 2.197931000 & 1.003496000 \\
\hline H & -5.337506000 & -2.551593000 & -0.244510000 & $\mathrm{H}$ & 6.471915000 & 2.427986000 & -0.708276000 \\
\hline $\mathrm{H}$ & -6.571679000 & 0.739285000 & 0.784974000 & $\mathrm{H}$ & 7.679082000 & -0.706264000 & 0.755121000 \\
\hline $\mathrm{H}$ & -6.514345000 & 0.461630000 & -0.957196000 & $\mathrm{H}$ & 7.577543000 & -0.682182000 & -1.007088000 \\
\hline $\mathrm{H}$ & -7.025770000 & -0.827138000 & 0.127553000 & $\mathrm{H}$ & 8.141257000 & 0.738933000 & -0.134178000 \\
\hline $\mathrm{H}$ & -2.238725000 & 3.339129000 & -0.136981000 & $\mathrm{H}$ & 3.287236000 & -3.327220000 & 0.325272000 \\
\hline $\mathrm{H}$ & -1.480422000 & 1.777050000 & 0.045543000 & $\mathrm{H}$ & 2.551805000 & -1.744889000 & 0.287026000 \\
\hline H & -2.571781000 & 2.364948000 & 1.316494000 & $\mathrm{H}$ & 3.664452000 & -2.152359000 & 1.609630000 \\
\hline H & -4.547344000 & 3.646049000 & -0.505218000 & $\mathrm{H}$ & 5.577162000 & -3.724718000 & -0.056603000 \\
\hline $\mathrm{H}$ & -5.163442000 & 2.608907000 & 0.780600000 & $\mathrm{H}$ & 6.234399000 & -2.529541000 & 1.061174000 \\
\hline $\mathrm{H}$ & -5.685247000 & 2.371392000 & -0.898944000 & $\mathrm{H}$ & 6.726807000 & -2.537195000 & -0.643495000 \\
\hline $\mathrm{H}$ & -3.392443000 & 1.711358000 & -1.565076000 & $\mathrm{H}$ & 4.426450000 & -1.940384000 & -1.353408000 \\
\hline$P$ & 3.134076000 & 0.073718000 & -0.131750000 & $\mathrm{P}$ & -2.034788000 & -0.012969000 & 0.009025000 \\
\hline C & 3.774516000 & 1.759996000 & -0.541315000 & C & -2.674842000 & -1.397752000 & -0.998824000 \\
\hline $\mathrm{H}$ & 3.421539000 & 1.980974000 & -1.554609000 & $\mathrm{H}$ & -1.154744000 & -1.146165000 & -2.519679000 \\
\hline $\mathrm{H}$ & 4.867809000 & 1.701949000 & -0.590173000 & C & -2.030281000 & -1.709535000 & -2.207556000 \\
\hline C & 3.327313000 & 2.854274000 & 0.436686000 & C & -2.512631000 & -2.740460000 & -3.012058000 \\
\hline $\mathrm{H}$ & 3.699926000 & 2.674325000 & 1.449226000 & C & -3.633843000 & -3.472425000 & -2.612784000 \\
\hline $\mathrm{H}$ & 2.236286000 & 2.928790000 & 0.484420000 & $\mathrm{H}$ & -2.010910000 & -2.975941000 & -3.945485000 \\
\hline H & 3.714174000 & 3.823886000 & 0.111799000 & $\mathrm{H}$ & -4.004642000 & -4.279762000 & -3.236796000 \\
\hline C & 3.863270000 & -1.042944000 & -1.411661000 & C & -4.274668000 & -3.169882000 & -1.410078000 \\
\hline $\mathrm{H}$ & 3.518270000 & -0.661001000 & -2.378843000 & $\mathrm{H}$ & -5.143773000 & -3.739979000 & -1.096831000 \\
\hline $\mathrm{H}$ & 4.951334000 & -0.916826000 & -1.388778000 & C & -3.800128000 & -2.135497000 & -0.601812000 \\
\hline $\mathrm{H}$ & 3.839040000 & -2.934247000 & -0.302327000 & $\mathrm{H}$ & -4.300634000 & -1.910004000 & 0.334153000 \\
\hline C & 3.477646000 & -2.518971000 & -1.247696000 & C & -1.994645000 & -1.030139000 & 2.609725000 \\
\hline H & 2.392161000 & -2.655018000 & -1.286764000 & $\mathrm{H}$ & -1.066033000 & -1.509261000 & 2.310597000 \\
\hline H & 3.460145000 & -1.405388000 & 1.728863000 & C & -2.491298000 & -1.227146000 & 3.897184000 \\
\hline C & 3.853487000 & -0.407987000 & 1.504535000 & $\mathrm{H}$ & -1.948453000 & -1.858113000 & 4.594049000 \\
\hline $\mathrm{H}$ & 3.402639000 & 0.264741000 & 2.242234000 & C & -3.679489000 & -0.606482000 & 4.290579000 \\
\hline $\mathrm{H}$ & 3.917243000 & -3.110232000 & -2.055489000 & $\mathrm{H}$ & -4.061553000 & -0.755044000 & 5.295868000 \\
\hline $\mathrm{H}$ & 5.850401000 & -1.084374000 & 0.900219000 & C & -4.372892000 & 0.208977000 & 3.394338000 \\
\hline C & 5.384612000 & -0.385675000 & 1.600927000 & $\mathrm{H}$ & -5.294143000 & 0.695171000 & 3.699934000 \\
\hline H & 5.692752000 & -0.679557000 & 2.608298000 & C & -3.883561000 & 0.406915000 & 2.102269000 \\
\hline \multirow[t]{8}{*}{$\mathrm{H}$} & 5.793648000 & 0.610961000 & 1.412164000 & $\mathrm{H}$ & -4.424379000 & 1.047608000 & 1.413224000 \\
\hline & & & & C & -2.690706000 & -0.214791000 & 1.702552000 \\
\hline & & & & C & -2.747656000 & 1.531225000 & -0.664515000 \\
\hline & & & & C & -3.840656000 & 1.516484000 & -1.542736000 \\
\hline & & & & $\mathrm{H}$ & -4.277423000 & 0.574661000 & -1.858080000 \\
\hline & & & & C & -4.366436000 & 2.719009000 & -2.019600000 \\
\hline & & & & $\mathrm{H}$ & -5.210861000 & 2.702181000 & -2.701640000 \\
\hline & & & & C & -3.809193000 & 3.935683000 & -1.623780000 \\
\hline
\end{tabular}




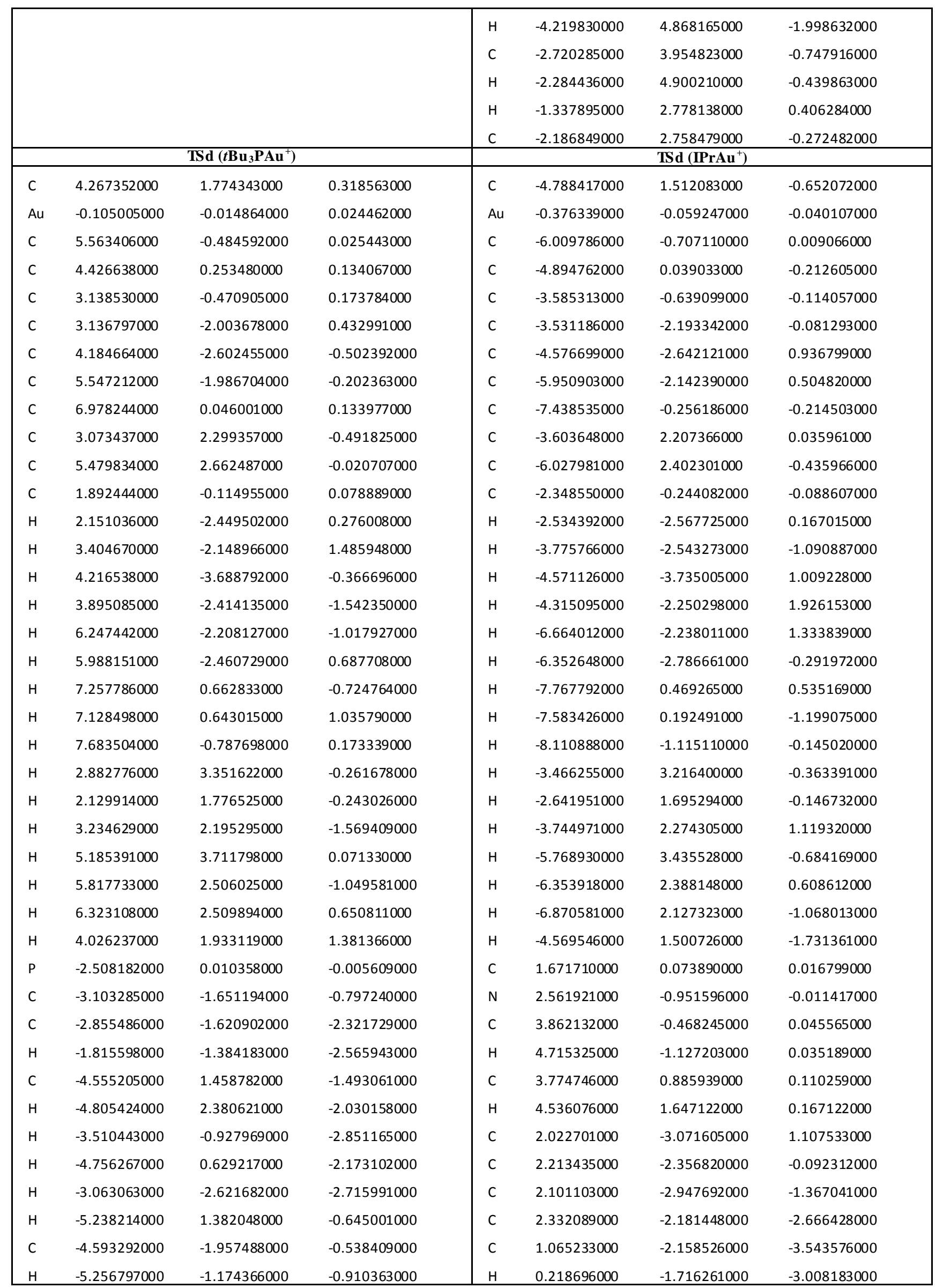




\begin{tabular}{|c|c|c|c|c|c|c|c|}
\hline $\mathrm{H}$ & -4.853945000 & -2.882289000 & -1.065458000 & $\mathrm{H}$ & 1.241668000 & -1.568299000 & -4.448584000 \\
\hline $\mathrm{H}$ & -4.812208000 & -2.120338000 & 0.518499000 & $\mathrm{H}$ & 0.775457000 & -3.166419000 & -3.857883000 \\
\hline $\mathrm{H}$ & -5.274155000 & -0.132779000 & 1.451945000 & $\mathrm{H}$ & 2.569634000 & -1.143165000 & -2.415830000 \\
\hline C & -4.602368000 & 0.571597000 & 1.946522000 & $\mathrm{H}$ & 3.717550000 & -2.155206000 & -4.347519000 \\
\hline c & -2.235922000 & -2.809010000 & -0.246546000 & C & 3.535868000 & -2.748185000 & -3.445507000 \\
\hline $\mathrm{H}$ & -2.557647000 & -3.736597000 & -0.733279000 & $\mathrm{H}$ & 4.448151000 & -2.734211000 & -2.840911000 \\
\hline H & -2.328155000 & -2.956584000 & 0.827538000 & $\mathrm{H}$ & 3.360915000 & -3.782453000 & -3.758567000 \\
\hline $\mathrm{H}$ & -3.563571000 & -1.963539000 & 2.220152000 & $\mathrm{H}$ & 1.673943000 & -4.799152000 & -2.375970000 \\
\hline $\mathrm{H}$ & -3.111821000 & -1.008878000 & 3.624707000 & C & 1.769139000 & -4.307514000 & -1.412979000 \\
\hline C & -2.898637000 & -1.169407000 & 2.562311000 & C & 1.565112000 & -5.040264000 & -0.246946000 \\
\hline $\mathrm{H}$ & -1.863150000 & -1.515484000 & 2.488416000 & $\mathrm{H}$ & 1.312892000 & -6.094791000 & -0.307819000 \\
\hline $\mathrm{H}$ & -2.561982000 & 1.250267000 & 3.605513000 & C & 1.692934000 & -4.428230000 & 0.996961000 \\
\hline c & -2.240666000 & 1.212996000 & 2.558605000 & $\mathrm{H}$ & 1.541298000 & -5.013365000 & 1.898600000 \\
\hline H & -1.183518000 & 0.930457000 & 2.549263000 & $\mathrm{H}$ & 4.275633000 & -3.018997000 & 2.732800000 \\
\hline $\mathrm{H}$ & -2.328525000 & 2.220483000 & 2.157028000 & C & 3.328829000 & -3.086684000 & 3.277704000 \\
\hline $\mathrm{H}$ & -1.177441000 & -2.663331000 & -0.484198000 & $\mathrm{H}$ & 3.134946000 & -4.145686000 & 3.475707000 \\
\hline c & -3.117661000 & 0.169778000 & 1.824663000 & $\mathrm{H}$ & 3.456387000 & -2.586583000 & 4.243051000 \\
\hline $\mathrm{H}$ & -4.801478000 & 1.570384000 & 1.553373000 & C & 0.856458000 & -2.495622000 & 3.285161000 \\
\hline H & -4.872045000 & 0.586695000 & 3.008543000 & $\mathrm{H}$ & 0.043740000 & -1.999967000 & 2.743932000 \\
\hline C & -3.075266000 & 1.529438000 & -1.064260000 & $\mathrm{H}$ & 0.549807000 & -3.527893000 & 3.482989000 \\
\hline C & -2.846701000 & 2.832902000 & -0.267550000 & $\mathrm{H}$ & 0.976166000 & -1.995728000 & 4.251760000 \\
\hline $\mathrm{H}$ & -3.041509000 & 3.676627000 & -0.938512000 & C & 2.174010000 & -2.439076000 & 2.487797000 \\
\hline $\mathrm{H}$ & -1.814050000 & 2.927949000 & 0.081665000 & $\mathrm{H}$ & 2.424796000 & -1.382296000 & 2.355478000 \\
\hline $\mathrm{H}$ & -3.519265000 & 2.940634000 & 0.584139000 & $\mathrm{~N}$ & 2.423256000 & 1.202128000 & 0.091969000 \\
\hline C & -2.177503000 & 1.631048000 & -2.320758000 & C & 1.898682000 & 2.553052000 & 0.143433000 \\
\hline H & -2.268375000 & 0.783553000 & -2.997188000 & C & 1.678920000 & 3.234047000 & -1.071015000 \\
\hline $\mathrm{H}$ & -2.473051000 & 2.526491000 & -2.879088000 & C & 1.654450000 & 3.131071000 & 1.405583000 \\
\hline \multirow[t]{19}{*}{$\mathrm{H}$} & -1.122027000 & 1.743748000 & -2.054395000 & C & 1.916076000 & 2.403964000 & 2.721713000 \\
\hline & & & & $\mathrm{H}$ & 2.301325000 & 1.405895000 & 2.492031000 \\
\hline & & & & $\mathrm{H}$ & -0.130938000 & 1.660164000 & 2.957162000 \\
\hline & & & & C & 0.618576000 & 2.214683000 & 3.531264000 \\
\hline & & & & $\mathrm{H}$ & 0.179280000 & 3.175812000 & 3.817040000 \\
\hline & & & & $\mathrm{H}$ & 0.823426000 & 1.657939000 & 4.451419000 \\
\hline & & & & $\mathrm{H}$ & 3.201344000 & 2.565377000 & 4.473632000 \\
\hline & & & & C & 2.992151000 & 3.126000000 & 3.556990000 \\
\hline & & & & $\mathrm{H}$ & 3.929458000 & 3.229352000 & 3.001228000 \\
\hline & & & & $\mathrm{H}$ & 2.667309000 & 4.129646000 & 3.849105000 \\
\hline & & & & $\mathrm{H}$ & 0.957563000 & 4.920041000 & 2.375749000 \\
\hline & & & & C & 1.156313000 & 4.439838000 & 1.422776000 \\
\hline & & & & $\mathrm{H}$ & 0.537139000 & 6.152664000 & 0.279573000 \\
\hline & & & & C & 0.919249000 & 5.136886000 & 0.241229000 \\
\hline & & & & $\mathrm{H}$ & 0.999654000 & 5.097912000 & -1.902664000 \\
\hline & & & & C & 1.179603000 & 4.539757000 & -0.989180000 \\
\hline & & & & $\mathrm{H}$ & 2.337548000 & 1.600501000 & -2.284053000 \\
\hline & & & & C & 1.973248000 & 2.620769000 & -2.437249000 \\
\hline & & & & C & 3.085215000 & 3.396832000 & -3.170901000 \\
\hline
\end{tabular}




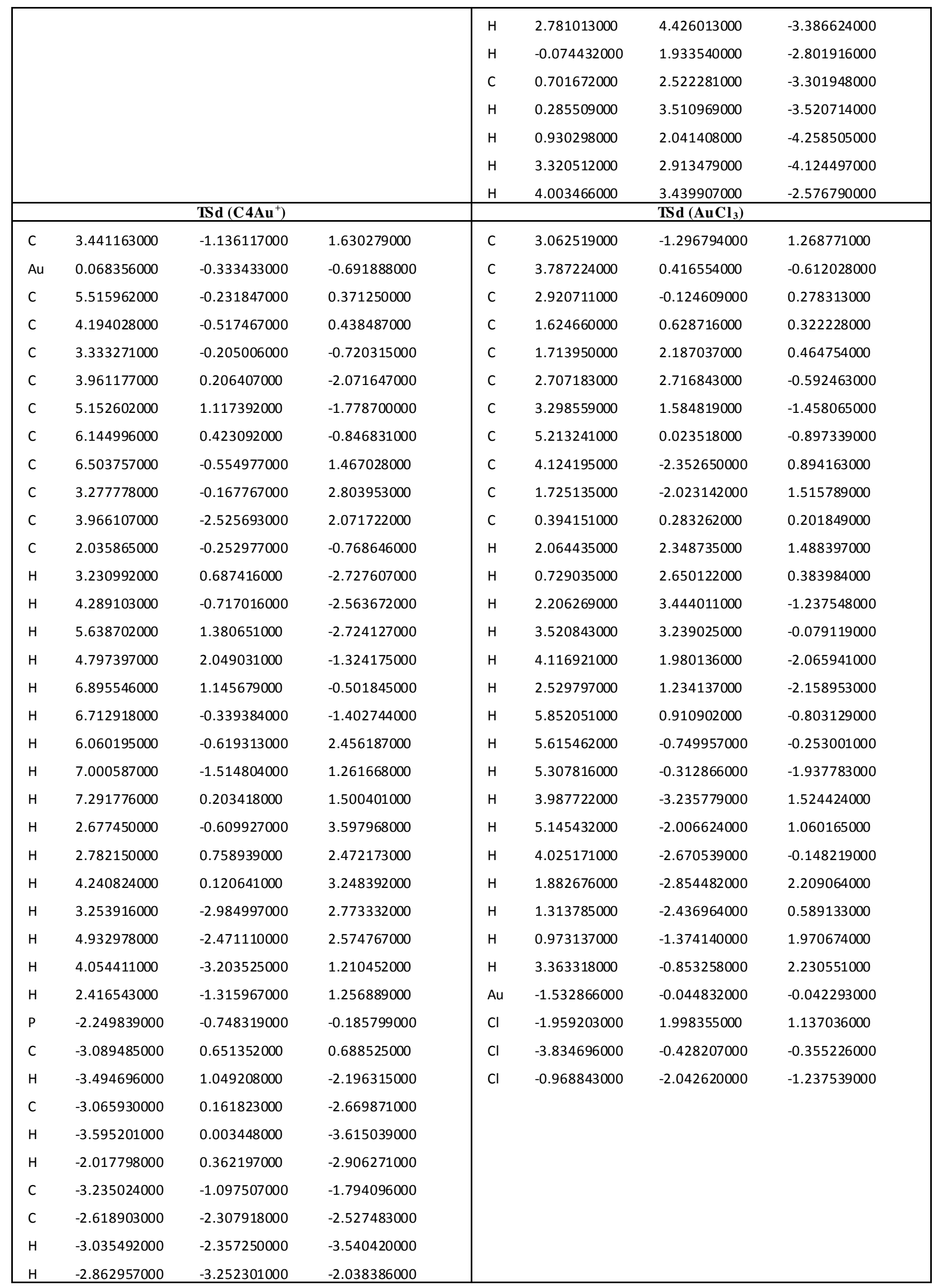




\begin{tabular}{|c|c|c|c|c|c|c|c|}
\hline H & -1.818681000 & -3.855574000 & -0.354339000 & & & & \\
\hline $\mathrm{H}$ & -1.092190000 & -4.084868000 & 1.238264000 & & & & \\
\hline c & -1.295295000 & -3.341650000 & 0.457230000 & & & & \\
\hline $\mathrm{H}$ & -1.530835000 & -2.232834000 & -2.617747000 & & & & \\
\hline $\mathrm{H}$ & -0.329131000 & -2.989848000 & 0.075488000 & & & & \\
\hline $\mathrm{H}$ & -0.449688000 & -2.125551000 & 2.493451000 & & & & \\
\hline C & -1.394353000 & -1.614532000 & 2.317034000 & & & & \\
\hline $\mathrm{H}$ & -1.164897000 & -0.549913000 & 2.231247000 & & & & \\
\hline $\mathrm{H}$ & -1.997099000 & -1.739552000 & 3.216097000 & & & & \\
\hline C & -2.139615000 & -2.203807000 & 1.077010000 & & & & \\
\hline C & -3.484698000 & -2.806691000 & 1.522747000 & & & & \\
\hline $\mathrm{H}$ & -3.275586000 & -3.627792000 & 2.215719000 & & & & \\
\hline $\mathrm{H}$ & -4.053135000 & -3.228172000 & 0.691921000 & & & & \\
\hline H & -4.108743000 & -2.089207000 & 2.056645000 & & & & \\
\hline $\mathrm{H}$ & -4.945691000 & -2.206673000 & -0.957911000 & & & & \\
\hline H & -5.195291000 & -1.522505000 & -2.565360000 & & & & \\
\hline $\mathrm{H}$ & -5.244051000 & -0.466594000 & -1.160529000 & & & & \\
\hline C & -4.740290000 & -1.338978000 & -1.585395000 & & & & \\
\hline H & -4.869288000 & -0.528145000 & 1.026371000 & & & & \\
\hline C & -4.377409000 & 0.415613000 & 1.214210000 & & & & \\
\hline C & -5.050421000 & 1.363489000 & 1.980529000 & & & & \\
\hline $\mathrm{H}$ & -6.040189000 & 1.140735000 & 2.368459000 & & & & \\
\hline $\mathrm{H}$ & -4.943375000 & 3.335088000 & 2.853747000 & & & & \\
\hline C & -4.440743000 & 2.587620000 & 2.245000000 & & & & \\
\hline C & -3.180668000 & 2.852102000 & 1.715539000 & & & & \\
\hline $\mathrm{H}$ & -2.715643000 & 3.815702000 & 1.894650000 & & & & \\
\hline C & -2.486544000 & 1.914614000 & 0.928393000 & & & & \\
\hline C & -0.103736000 & 2.636837000 & 1.287693000 & & & & \\
\hline C & -1.167206000 & 2.390458000 & 0.400801000 & & & & \\
\hline $\mathrm{H}$ & -0.211723000 & 2.383818000 & 2.340787000 & & & & \\
\hline C & -1.017925000 & 2.761325000 & -0.947725000 & & & & \\
\hline $\mathrm{H}$ & -1.846674000 & 2.631508000 & -1.636385000 & & & & \\
\hline C & 0.169864000 & 3.343235000 & -1.400877000 & & & & \\
\hline $\mathrm{H}$ & 0.253958000 & 3.655078000 & -2.438004000 & & & & \\
\hline C & 1.218226000 & 3.583703000 & -0.509507000 & & & & \\
\hline H & 2.126940000 & 4.068606000 & -0.854014000 & & & & \\
\hline C & 1.080438000 & 3.225067000 & 0.833709000 & & & & \\
\hline $\mathrm{H}$ & 1.887143000 & 3.420463000 & 1.535708000 & & & & \\
\hline \multicolumn{4}{|c|}{ Int5 $\left(\mathrm{H}_{3} \mathrm{PAu}^{+}\right)$} & \multicolumn{4}{|c|}{ Int5 $\left(\mathrm{Me}_{3} \mathrm{PAu}^{+}\right)$} \\
\hline C & 2.531365000 & 1.873244000 & -0.150538000 & C & 3.108025000 & 1.877393000 & -0.143401000 \\
\hline $\mathrm{Au}$ & -1.765569000 & -0.079577000 & -0.002268000 & $\mathrm{Au}$ & -1.188153000 & -0.095185000 & 0.008327000 \\
\hline C & 3.898855000 & -0.292728000 & -0.117698000 & C & 4.489591000 & -0.277655000 & -0.124256000 \\
\hline C & 2.709871000 & 0.359158000 & -0.068524000 & C & 3.296276000 & 0.364555000 & -0.066736000 \\
\hline C & 1.477490000 & -0.418506000 & 0.123252000 & C & 2.069520000 & -0.424469000 & 0.128777000 \\
\hline C & 1.590299000 & -1.911277000 & 0.585965000 & C & 2.199338000 & -1.914098000 & 0.588724000 \\
\hline C & 2.696840000 & -2.550791000 & -0.254320000 & $\mathrm{C}$ & 3.304174000 & -2.545505000 & -0.259759000 \\
\hline
\end{tabular}




\begin{tabular}{|c|c|c|c|c|c|c|c|}
\hline c & 4.013493000 & -1.802230000 & -0.058166000 & C & 4.616546000 & -1.786896000 & -0.071771000 \\
\hline c & 5.226209000 & 0.406795000 & -0.247330000 & C & 5.813145000 & 0.429952000 & -0.255575000 \\
\hline c & 2.392814000 & 2.497249000 & 1.251837000 & c & 2.940119000 & 2.493127000 & 1.259482000 \\
\hline C & 1.357186000 & 2.263217000 & -1.052919000 & C & 1.946919000 & 2.266033000 & -1.064793000 \\
\hline c & 0.212567000 & -0.168177000 & 0.030137000 & C & 0.801753000 & -0.183297000 & 0.038319000 \\
\hline $\mathrm{H}$ & 0.646779000 & -2.453737000 & 0.486356000 & $\mathrm{H}$ & 1.258712000 & -2.462923000 & 0.496390000 \\
\hline $\mathrm{H}$ & 1.849549000 & -1.881902000 & 1.649823000 & H & 2.467992000 & -1.886676000 & 1.650504000 \\
\hline $\mathrm{H}$ & 2.801650000 & -3.598449000 & 0.047772000 & $\mathrm{H}$ & 3.420380000 & -3.593166000 & 0.038163000 \\
\hline $\mathrm{H}$ & 2.403954000 & -2.547274000 & -1.309915000 & H & 3.003804000 & -2.541125000 & -1.313391000 \\
\hline $\mathrm{H}$ & 4.744354000 & -2.125148000 & -0.809621000 & $\mathrm{H}$ & 5.343751000 & -2.101256000 & -0.830555000 \\
\hline $\mathrm{H}$ & 4.460127000 & -2.069399000 & 0.911291000 & $\mathrm{H}$ & 5.073103000 & -2.055048000 & 0.892837000 \\
\hline $\mathrm{H}$ & 5.612352000 & 0.300990000 & -1.269583000 & $\mathrm{H}$ & 6.199525000 & 0.327764000 & -1.278100000 \\
\hline $\mathrm{H}$ & 5.193993000 & 1.468367000 & -0.003939000 & H & 5.775749000 & 1.491079000 & -0.010858000 \\
\hline H & 5.961867000 & -0.068534000 & 0.411029000 & H & 6.552299000 & -0.041339000 & 0.401970000 \\
\hline $\mathrm{H}$ & 2.293369000 & 3.584785000 & 1.181434000 & H & 2.833039000 & 3.580259000 & 1.192490000 \\
\hline $\mathrm{H}$ & 3.265080000 & 2.275943000 & 1.873349000 & $\mathrm{H}$ & 3.803658000 & 2.275772000 & 1.894559000 \\
\hline $\mathrm{H}$ & 1.506601000 & 2.112896000 & 1.769362000 & $\mathrm{H}$ & 2.048316000 & 2.099093000 & 1.759798000 \\
\hline $\mathrm{H}$ & 1.258441000 & 3.351438000 & -1.110788000 & $\mathrm{H}$ & 1.850642000 & 3.354515000 & -1.123909000 \\
\hline $\mathrm{H}$ & 0.396762000 & 1.900503000 & -0.641955000 & H & 0.981189000 & 1.900066000 & -0.673569000 \\
\hline $\mathrm{H}$ & 1.461154000 & 1.866381000 & -2.066510000 & $\mathrm{H}$ & 2.071307000 & 1.870938000 & -2.076919000 \\
\hline $\mathrm{H}$ & 3.431930000 & 2.281177000 & -0.618755000 & H & 4.013713000 & 2.295436000 & -0.591946000 \\
\hline $\mathrm{H}$ & -4.792307000 & -0.627043000 & -1.100468000 & $\mathrm{P}$ & -3.564043000 & 0.003638000 & -0.020805000 \\
\hline$P$ & -4.143376000 & 0.014491000 & -0.031049000 & C & -4.335378000 & -1.352115000 & -0.987803000 \\
\hline $\mathrm{H}$ & -4.704906000 & 1.302272000 & -0.084462000 & $\mathrm{H}$ & -4.056766000 & -2.317130000 & -0.557475000 \\
\hline \multirow[t]{11}{*}{$\mathrm{H}$} & -4.802054000 & -0.539743000 & 1.080314000 & $\mathrm{H}$ & -5.424998000 & -1.254486000 & -0.978444000 \\
\hline & & & & $\mathrm{H}$ & -3.978777000 & -1.315959000 & -2.020034000 \\
\hline & & & & C & -4.192440000 & 1.560205000 & -0.764318000 \\
\hline & & & & $\mathrm{H}$ & -5.286468000 & 1.568847000 & -0.759017000 \\
\hline & & & & $\mathrm{H}$ & -3.822711000 & 2.416240000 & -0.194655000 \\
\hline & & & & $\mathrm{H}$ & -3.836051000 & 1.646618000 & -1.793635000 \\
\hline & & & & C & -4.318719000 & -0.086577000 & 1.649406000 \\
\hline & & & & H & -4.043123000 & -1.029673000 & 2.127593000 \\
\hline & & & & H & -3.948141000 & 0.736174000 & 2.265604000 \\
\hline & & & & $\mathrm{H}$ & -5.408620000 & -0.022927000 & 1.579051000 \\
\hline & \multicolumn{3}{|c|}{ Int5 $\left(\mathrm{Et}_{3} \mathrm{PAu}^{+}\right)$} & \multicolumn{4}{|c|}{ Int5 $\left(\mathrm{Ph}_{3} \mathrm{PAu}^{+}\right)$} \\
\hline C & 3.605323000 & 1.820603000 & 0.253342000 & C & 4.662349000 & -1.850402000 & 0.160647000 \\
\hline $\mathrm{Au}$ & -0.712553000 & -0.085142000 & 0.055375000 & $\mathrm{Au}$ & 0.382372000 & 0.130052000 & 0.001588000 \\
\hline C & 4.956403000 & -0.283467000 & -0.290082000 & C & 6.057222000 & 0.294435000 & 0.125795000 \\
\hline C & 3.774413000 & 0.332097000 & -0.037926000 & c & 4.859807000 & -0.339811000 & 0.073809000 \\
\hline C & 2.544267000 & -0.476513000 & 0.008978000 & C & 3.637823000 & 0.457832000 & -0.125582000 \\
\hline C & 2.671614000 & -2.031816000 & 0.097578000 & C & 3.779224000 & 1.941676000 & -0.596355000 \\
\hline C & 3.738337000 & -2.448848000 & -0.916007000 & C & 4.888519000 & 2.572123000 & 0.247163000 \\
\hline C & 5.066000000 & -1.762853000 & -0.600323000 & C & 6.195283000 & 1.802324000 & 0.065417000 \\
\hline C & 6.284170000 & 0.428651000 & -0.299132000 & C & 7.376729000 & -0.421999000 & 0.259002000 \\
\hline C & 3.494309000 & 2.086934000 & 1.767368000 & C & 4.464702000 & -2.469304000 & -1.237170000 \\
\hline C & 2.418854000 & 2.424312000 & -0.505963000 & C & 3.516169000 & -2.230149000 & 1.105229000 \\
\hline
\end{tabular}




\begin{tabular}{|c|c|c|c|c|c|c|c|}
\hline c & 1.277696000 & -0.211342000 & 0.025138000 & C & 2.368663000 & 0.223998000 & -0.031303000 \\
\hline $\mathrm{H}$ & 1.720904000 & -2.538325000 & -0.087326000 & $\mathrm{H}$ & 2.842168000 & 2.497103000 & -0.508454000 \\
\hline $\mathrm{H}$ & 2.978148000 & -2.258744000 & 1.124708000 & $\mathrm{H}$ & 4.048096000 & 1.905541000 & -1.657805000 \\
\hline $\mathrm{H}$ & 3.851064000 & -3.537840000 & -0.881068000 & $\mathrm{H}$ & 5.013087000 & 3.616751000 & -0.057952000 \\
\hline $\mathrm{H}$ & 3.402775000 & -2.191018000 & -1.926605000 & $\mathrm{H}$ & 4.587522000 & 2.577127000 & 1.300573000 \\
\hline $\mathrm{H}$ & 5.763769000 & -1.892299000 & -1.436906000 & $\mathrm{H}$ & 6.923778000 & 2.115325000 & 0.823734000 \\
\hline H & 5.550468000 & -2.253671000 & 0.257224000 & $\mathrm{H}$ & 6.655877000 & 2.062018000 & -0.899747000 \\
\hline $\mathrm{H}$ & 6.633731000 & 0.568741000 & -1.330374000 & $\mathrm{H}$ & 7.749972000 & -0.346829000 & 1.288690000 \\
\hline $\mathrm{H}$ & 6.270019000 & 1.402330000 & 0.189850000 & $\mathrm{H}$ & 7.337052000 & -1.477076000 & -0.011034000 \\
\hline $\mathrm{H}$ & 7.039178000 & -0.188029000 & 0.201390000 & $\mathrm{H}$ & 8.126705000 & 0.059889000 & -0.377906000 \\
\hline $\mathrm{H}$ & 3.393433000 & 3.158997000 & 1.963197000 & $\mathrm{H}$ & 4.354381000 & -3.555750000 & -1.164778000 \\
\hline $\mathrm{H}$ & 4.378635000 & 1.726190000 & 2.300395000 & $\mathrm{H}$ & 5.316469000 & -2.256615000 & -1.889459000 \\
\hline H & 2.618234000 & 1.584892000 & 2.193096000 & $\mathrm{H}$ & 3.564530000 & -2.073542000 & -1.720658000 \\
\hline H & 2.341502000 & 3.497680000 & -0.306950000 & $\mathrm{H}$ & 3.425807000 & -3.318174000 & 1.179163000 \\
\hline $\mathrm{H}$ & 1.461821000 & 1.986374000 & -0.172533000 & $\mathrm{H}$ & 2.544020000 & -1.871436000 & 0.726866000 \\
\hline $\mathrm{H}$ & 2.499902000 & 2.274785000 & -1.586319000 & $\mathrm{H}$ & 3.657849000 & -1.822987000 & 2.110366000 \\
\hline $\mathrm{H}$ & 4.500876000 & 2.328077000 & -0.116054000 & $\mathrm{H}$ & 5.574179000 & -2.272868000 & 0.592336000 \\
\hline$P$ & -3.086629000 & 0.082199000 & 0.109215000 & $\mathrm{P}$ & -2.000227000 & 0.009769000 & 0.014180000 \\
\hline C & -3.655295000 & 1.840556000 & 0.184054000 & C & -2.739540000 & 1.468603000 & -0.804398000 \\
\hline $\mathrm{H}$ & -3.268222000 & 2.245852000 & 1.125423000 & C & -2.190168000 & 2.736634000 & -0.551522000 \\
\hline $\mathrm{H}$ & -4.747926000 & 1.834739000 & 0.265554000 & $\mathrm{H}$ & -1.329780000 & 2.837199000 & 0.105246000 \\
\hline C & -3.196750000 & 2.698872000 & -1.002121000 & $\mathrm{H}$ & -2.321578000 & 4.848142000 & -0.937366000 \\
\hline $\mathrm{H}$ & -3.603128000 & 2.336045000 & -1.950638000 & C & -2.749464000 & 3.870689000 & -1.137399000 \\
\hline $\mathrm{H}$ & -2.105437000 & 2.718481000 & -1.085670000 & C & -3.853923000 & 3.747465000 & -1.984613000 \\
\hline H & -3.539906000 & 3.728532000 & -0.870240000 & $\mathrm{H}$ & -4.285003000 & 4.630960000 & -2.445283000 \\
\hline C & -3.827865000 & -0.726844000 & 1.597189000 & $\mathrm{H}$ & -5.256719000 & 2.391629000 & -2.902426000 \\
\hline $\mathrm{H}$ & -3.447116000 & -0.173449000 & 2.462708000 & C & -4.400428000 & 2.489436000 & -2.242452000 \\
\hline $\mathrm{H}$ & -4.911055000 & -0.563276000 & 1.565356000 & C & -3.847670000 & 1.349351000 & -1.655727000 \\
\hline $\mathrm{H}$ & -3.902619000 & -2.801579000 & 0.891999000 & $\mathrm{H}$ & -4.275130000 & 0.374269000 & -1.865481000 \\
\hline C & -3.500224000 & -2.220041000 & 1.726650000 & C & -2.687648000 & -0.067376000 & 1.705192000 \\
\hline H & -2.419664000 & -2.389503000 & 1.770713000 & C & -3.912855000 & 0.538920000 & 2.022261000 \\
\hline H & -3.529895000 & -1.726298000 & -1.407161000 & $\mathrm{H}$ & -4.460008000 & 1.094203000 & 1.267269000 \\
\hline C & -3.863947000 & -0.683254000 & -1.386875000 & C & -4.426182000 & 0.435599000 & 3.315885000 \\
\hline $\mathrm{H}$ & -3.396069000 & -0.200803000 & -2.251899000 & $\mathrm{H}$ & -5.372533000 & 0.909521000 & 3.557417000 \\
\hline $\mathrm{H}$ & -3.938069000 & -2.618766000 & 2.645701000 & C & -3.724821000 & -0.269273000 & 4.295713000 \\
\hline $\mathrm{H}$ & -5.879392000 & -1.107472000 & -0.631630000 & $\mathrm{H}$ & -4.125867000 & -0.343977000 & 5.301769000 \\
\hline C & -5.393915000 & -0.592928000 & -1.465771000 & $\mathrm{H}$ & -1.954990000 & -1.418060000 & 4.746807000 \\
\hline $\mathrm{H}$ & -5.742180000 & -1.065660000 & -2.388408000 & C & -2.503997000 & -0.873571000 & 3.984882000 \\
\hline \multirow[t]{8}{*}{$\mathrm{H}$} & -5.743024000 & 0.443517000 & -1.476940000 & C & -1.983406000 & -0.771053000 & 2.695944000 \\
\hline & & & & $\mathrm{H}$ & -1.030005000 & -1.236749000 & 2.460594000 \\
\hline & & & & C & -2.580418000 & -1.472251000 & -0.886444000 \\
\hline & & & & C & -1.916288000 & -1.847491000 & -2.066275000 \\
\hline & & & & $\mathrm{H}$ & -1.062239000 & -1.274205000 & -2.417489000 \\
\hline & & & & $\mathrm{H}$ & -1.834524000 & -3.238997000 & -3.703594000 \\
\hline & & & & C & -2.350989000 & -2.955025000 & -2.792015000 \\
\hline & & & & C & -3.443283000 & -3.701002000 & -2.341517000 \\
\hline
\end{tabular}




\begin{tabular}{|c|c|c|c|c|c|c|c|}
\hline & & & & $\mathrm{H}$ & -3.776811000 & -4.567796000 & -2.903709000 \\
\hline & & & & $\mathrm{H}$ & -4.948895000 & -3.916593000 & -0.813100000 \\
\hline & & & & c & -4.102499000 & -3.335941000 & -1.166681000 \\
\hline & & & & C & -3.676245000 & -2.224251000 & -0.437466000 \\
\hline & & & & H & -4.191379000 & -1.949747000 & 0.477120000 \\
\hline & & Int5 $\left(t \mathrm{Bu}_{3} \mathbf{P A u ^ { + }}\right)$ & & & & Int5 $\left(\right.$ IPrAu $\left.{ }^{+}\right)$ & \\
\hline C & 4.225607000 & -1.870369000 & 0.136873000 & C & -4.789647000 & 1.572275000 & -0.609507000 \\
\hline $\mathrm{Au}$ & -0.067091000 & 0.107940000 & -0.036673000 & $\mathrm{Au}$ & -0.401293000 & -0.080711000 & -0.014863000 \\
\hline C & 5.608673000 & 0.281482000 & 0.145166000 & C & -6.043810000 & -0.506480000 & 0.194655000 \\
\hline C & 4.415288000 & -0.357709000 & 0.065495000 & c & -4.888330000 & 0.131713000 & -0.115825000 \\
\hline c & 3.192843000 & 0.436211000 & -0.150273000 & c & -3.616493000 & -0.606732000 & -0.019294000 \\
\hline C & 3.339009000 & 1.921568000 & -0.611667000 & C & -3.662032000 & -2.161595000 & 0.108241000 \\
\hline c & 4.427203000 & 2.553494000 & 0.257360000 & c & -4.734793000 & -2.494422000 & 1.145957000 \\
\hline C & 5.741112000 & 1.790765000 & 0.098058000 & C & -6.086340000 & -1.931854000 & 0.709425000 \\
\hline C & 6.930124000 & -0.427053000 & 0.297129000 & C & -7.408899000 & 0.121170000 & 0.075019000 \\
\hline C & 4.052440000 & -2.481057000 & -1.267578000 & C & -4.616133000 & 1.629203000 & -2.140006000 \\
\hline C & 3.068341000 & -2.264000000 & 1.062454000 & C & -3.680955000 & 2.353482000 & 0.106068000 \\
\hline C & 1.922406000 & 0.197633000 & -0.075026000 & C & -2.365202000 & -0.272650000 & -0.033306000 \\
\hline $\mathrm{H}$ & 2.397907000 & 2.472793000 & -0.541774000 & $\mathrm{H}$ & -2.691308000 & -2.582526000 & 0.382485000 \\
\hline H & 3.631308000 & 1.892000000 & -1.667371000 & H & -3.925576000 & -2.549768000 & -0.882222000 \\
\hline $\mathrm{H}$ & 4.553323000 & 3.600543000 & -0.038740000 & $\mathrm{H}$ & -4.792073000 & -3.582151000 & 1.262393000 \\
\hline $\mathrm{H}$ & 4.105023000 & 2.550882000 & 1.304732000 & $\mathrm{H}$ & -4.442594000 & -2.080578000 & 2.117636000 \\
\hline H & 6.452222000 & 2.101930000 & 0.873405000 & H & -6.799266000 & -1.980270000 & 1.542128000 \\
\hline H & 6.219925000 & 2.059414000 & -0.855614000 & H & -6.521450000 & -2.564948000 & -0.078633000 \\
\hline $\mathrm{H}$ & 7.302056000 & -0.324618000 & 1.324971000 & $\mathrm{H}$ & -7.803104000 & 0.369475000 & 1.069148000 \\
\hline $\mathrm{H}$ & 6.896597000 & -1.488300000 & 0.052378000 & H & -7.433128000 & 1.024003000 & -0.534517000 \\
\hline $\mathrm{H}$ & 7.679139000 & 0.043199000 & -0.350083000 & $\mathrm{H}$ & -8.110168000 & -0.598105000 & -0.363474000 \\
\hline $\mathrm{H}$ & 3.942552000 & -3.568179000 & -1.203830000 & H & -4.573332000 & 2.667445000 & -2.484080000 \\
\hline $\mathrm{H}$ & 4.915387000 & -2.264024000 & -1.903694000 & H & -5.447529000 & 1.135939000 & -2.651934000 \\
\hline H & 3.160790000 & -2.082970000 & -1.764925000 & $\mathrm{H}$ & -3.689168000 & 1.134978000 & -2.451777000 \\
\hline $\mathrm{H}$ & 2.980101000 & -3.353245000 & 1.122359000 & $\mathrm{H}$ & -3.659186000 & 3.391209000 & -0.240945000 \\
\hline $\mathrm{H}$ & 2.100289000 & -1.901239000 & 0.677863000 & $\mathrm{H}$ & -2.683502000 & 1.939061000 & -0.114054000 \\
\hline $\mathrm{H}$ & 3.198101000 & -1.869109000 & 2.074204000 & $\mathrm{H}$ & -3.813121000 & 2.353085000 & 1.191893000 \\
\hline H & 5.132789000 & -2.291631000 & 0.578863000 & H & -5.731196000 & 2.068591000 & -0.358546000 \\
\hline$P$ & -2.470520000 & 0.002218000 & 0.016389000 & C & 1.648359000 & 0.067393000 & 0.025256000 \\
\hline C & -3.141929000 & 1.815359000 & -0.056474000 & N & 2.542917000 & -0.951967000 & -0.047414000 \\
\hline C & -2.914104000 & 2.512428000 & 1.303087000 & C & 3.841011000 & -0.465073000 & 0.021976000 \\
\hline $\mathrm{H}$ & -1.868671000 & 2.463778000 & 1.622375000 & $\mathrm{H}$ & 4.697066000 & -1.119182000 & -0.017833000 \\
\hline C & -4.473767000 & -0.673064000 & 2.026837000 & C & 3.747737000 & 0.885275000 & 0.139633000 \\
\hline H & -4.688466000 & -1.248095000 & 2.934666000 & H & 4.505803000 & 1.647383000 & 0.221959000 \\
\hline $\mathrm{H}$ & -3.545955000 & 2.118385000 & 2.100058000 & C & 2.020048000 & -3.114342000 & 0.996047000 \\
\hline $\mathrm{H}$ & -4.723865000 & 0.367375000 & 2.242540000 & C & 2.200744000 & -2.355019000 & -0.177808000 \\
\hline $\mathrm{H}$ & -3.169672000 & 3.571130000 & 1.185662000 & C & 2.085909000 & -2.899488000 & -1.472851000 \\
\hline H & -5.147485000 & -1.033991000 & 1.247325000 & C & 2.300454000 & -2.083057000 & -2.744104000 \\
\hline C & -4.639975000 & 1.897763000 & -0.415606000 & C & 1.023527000 & -2.032584000 & -3.605453000 \\
\hline $\mathrm{H}$ & -5.272206000 & 1.344700000 & 0.281796000 & $\mathrm{H}$ & 0.181218000 & -1.615638000 & -3.043623000 \\
\hline
\end{tabular}




\begin{tabular}{|c|c|c|c|c|c|c|c|}
\hline $\mathrm{H}$ & -4.950186000 & 2.947951000 & -0.373820000 & $\mathrm{H}$ & 1.186963000 & -1.407111000 & -4.489004000 \\
\hline $\mathrm{H}$ & -4.849972000 & 1.543238000 & -1.426459000 & $\mathrm{H}$ & 0.734547000 & -3.029102000 & -3.954730000 \\
\hline $\mathrm{H}$ & -5.221762000 & -0.665634000 & -1.319106000 & $\mathrm{H}$ & 2.535865000 & -1.053984000 & -2.456175000 \\
\hline C & -4.517204000 & -1.491921000 & -1.431541000 & $\mathrm{H}$ & 3.666513000 & -1.983271000 & -4.437429000 \\
\hline c & -2.318308000 & 2.616672000 & -1.093809000 & C & 3.498096000 & -2.612700000 & -3.557878000 \\
\hline $\mathrm{H}$ & -2.677782000 & 3.651820000 & -1.087564000 & $\mathrm{H}$ & 4.417009000 & -2.617872000 & -2.963271000 \\
\hline H & -2.412838000 & 2.246511000 & -2.112834000 & $\mathrm{H}$ & 3.324771000 & -3.634531000 & -3.910213000 \\
\hline $\mathrm{H}$ & -3.567115000 & 0.662627000 & -2.864355000 & $\mathrm{H}$ & 1.666197000 & -4.715674000 & -2.547045000 \\
\hline $\mathrm{H}$ & -3.067603000 & -0.820760000 & -3.663126000 & C & 1.763298000 & -4.259063000 & -1.567078000 \\
\hline C & -2.871494000 & -0.174880000 & -2.800427000 & C & 1.570221000 & -5.035408000 & -0.427710000 \\
\hline $\mathrm{H}$ & -1.849704000 & 0.202694000 & -2.904174000 & $\mathrm{H}$ & 1.325166000 & -6.088781000 & -0.526072000 \\
\hline $\mathrm{H}$ & -2.421526000 & -2.777988000 & -2.600071000 & C & 1.698924000 & -4.468291000 & 0.837235000 \\
\hline C & -2.124594000 & -2.249601000 & -1.687164000 & $\mathrm{H}$ & 1.554928000 & -5.087173000 & 1.717417000 \\
\hline H & -1.077909000 & -1.953035000 & -1.806616000 & $\mathrm{H}$ & 4.271365000 & -3.121923000 & 2.624280000 \\
\hline $\mathrm{H}$ & -2.186322000 & -2.962326000 & -0.867207000 & C & 3.324243000 & -3.207298000 & 3.166078000 \\
\hline $\mathrm{H}$ & -1.255205000 & 2.636917000 & -0.833824000 & $\mathrm{H}$ & 3.129312000 & -4.272229000 & 3.327823000 \\
\hline c & -3.051225000 & -1.020822000 & -1.520979000 & $\mathrm{H}$ & 3.451842000 & -2.740697000 & 4.148042000 \\
\hline $\mathrm{H}$ & -4.682530000 & -2.198706000 & -0.616405000 & C & 0.852056000 & -2.615574000 & 3.191144000 \\
\hline H & -4.771868000 & -2.011745000 & -2.362005000 & $\mathrm{H}$ & 0.040951000 & -2.097314000 & 2.668643000 \\
\hline C & -2.985256000 & -0.865093000 & 1.668616000 & $\mathrm{H}$ & 0.542923000 & -3.654112000 & 3.347912000 \\
\hline C & -2.683765000 & -2.376840000 & 1.574005000 & $\mathrm{H}$ & 0.971416000 & -2.153906000 & 4.176622000 \\
\hline $\mathrm{H}$ & -2.836083000 & -2.814922000 & 2.566437000 & C & 2.170865000 & -2.531470000 & 2.398145000 \\
\hline $\mathrm{H}$ & -1.645728000 & -2.572989000 & 1.288777000 & $\mathrm{H}$ & 2.422710000 & -1.470909000 & 2.303812000 \\
\hline $\mathrm{H}$ & -3.345621000 & -2.905712000 & 0.887070000 & $\mathrm{~N}$ & 2.394808000 & 1.195415000 & 0.140874000 \\
\hline C & -2.104297000 & -0.322177000 & 2.819642000 & C & 1.863927000 & 2.540780000 & 0.248437000 \\
\hline H & -2.233480000 & 0.741681000 & 3.008078000 & C & 1.648481000 & 3.272824000 & -0.936673000 \\
\hline $\mathrm{H}$ & -2.380944000 & -0.852451000 & 3.737797000 & C & 1.607596000 & 3.061064000 & 1.533138000 \\
\hline \multirow[t]{19}{*}{$\mathrm{H}$} & -1.041777000 & -0.511156000 & 2.638546000 & C & 1.859074000 & 2.276283000 & 2.817614000 \\
\hline & & & & $\mathrm{H}$ & 2.270273000 & 1.298612000 & 2.548385000 \\
\hline & & & & $\mathrm{H}$ & -0.177286000 & 1.481768000 & 2.971911000 \\
\hline & & & & C & 0.548740000 & 2.022253000 & 3.588394000 \\
\hline & & & & $\mathrm{H}$ & 0.084392000 & 2.960217000 & 3.909487000 \\
\hline & & & & $\mathrm{H}$ & 0.745396000 & 1.425931000 & 4.485350000 \\
\hline & & & & $\mathrm{H}$ & 3.106578000 & 2.371904000 & 4.600562000 \\
\hline & & & & C & 2.902061000 & 2.976450000 & 3.711228000 \\
\hline & & & & $\mathrm{H}$ & 3.846956000 & 3.130046000 & 3.180652000 \\
\hline & & & & $\mathrm{H}$ & 2.548921000 & 3.955318000 & 4.050653000 \\
\hline & & & & $\mathrm{H}$ & 0.893460000 & 4.802047000 & 2.575516000 \\
\hline & & & & C & 1.102276000 & 4.365297000 & 1.604052000 \\
\hline & & & & $\mathrm{H}$ & 0.481032000 & 6.123078000 & 0.532639000 \\
\hline & & & & C & 0.869581000 & 5.112208000 & 0.452536000 \\
\hline & & & & $\mathrm{H}$ & 0.962757000 & 5.167534000 & -1.690212000 \\
\hline & & & & C & 1.141348000 & 4.571055000 & -0.801037000 \\
\hline & & & & $\mathrm{H}$ & 2.330239000 & 1.699600000 & -2.216027000 \\
\hline & & & & C & 1.951136000 & 2.720074000 & -2.326634000 \\
\hline & & & & C & 3.051026000 & 3.540418000 & -3.029552000 \\
\hline
\end{tabular}




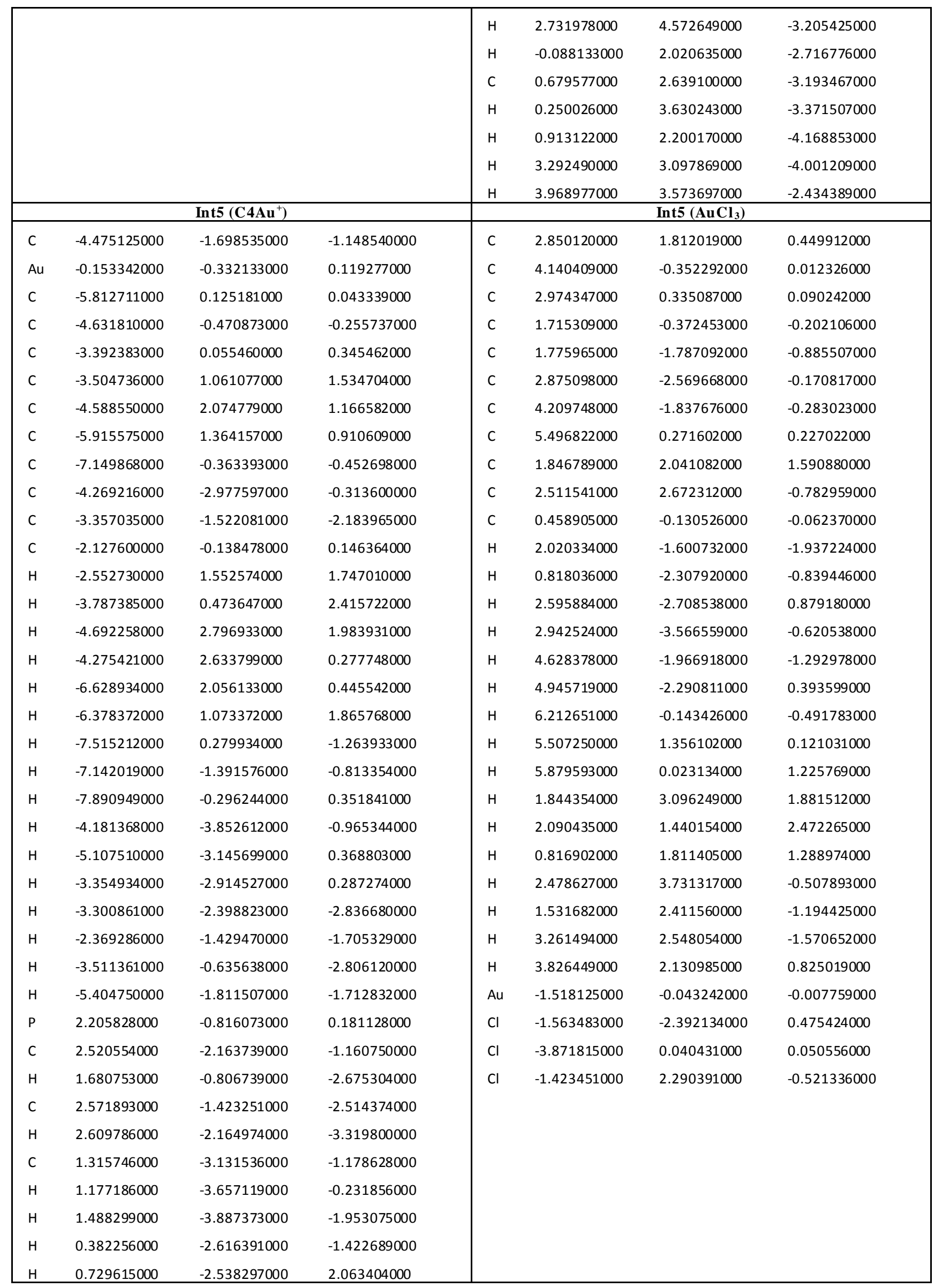




\begin{tabular}{|c|c|c|c|c|c|c|c|}
\hline c & 1.794921000 & -2.650806000 & 2.288694000 & & & & \\
\hline $\mathrm{H}$ & 2.174714000 & -3.521823000 & 1.748590000 & & & & \\
\hline $\mathrm{H}$ & 1.885596000 & -2.868476000 & 3.358583000 & & & & \\
\hline $\mathrm{H}$ & 3.788871000 & -3.582448000 & -0.051309000 & & & & \\
\hline c & 3.804619000 & -2.993777000 & -0.971709000 & & & & \\
\hline $\mathrm{H}$ & 3.880952000 & -3.701898000 & -1.804595000 & & & & \\
\hline $\mathrm{H}$ & 4.713464000 & -2.390061000 & -0.987845000 & & & & \\
\hline $\mathrm{H}$ & 4.532580000 & -2.381357000 & 1.635251000 & & & & \\
\hline C & 4.089081000 & -1.612278000 & 2.270345000 & & & & \\
\hline $\mathrm{H}$ & 4.182146000 & -1.954479000 & 3.307156000 & & & & \\
\hline $\mathrm{H}$ & 4.678206000 & -0.697649000 & 2.177705000 & & & & \\
\hline c & 2.596440000 & -1.371344000 & 1.974029000 & & & & \\
\hline C & 2.102659000 & -0.227238000 & 2.887770000 & & & & \\
\hline $\mathrm{H}$ & 2.299315000 & -0.498795000 & 3.930893000 & & & & \\
\hline $\mathrm{H}$ & 2.628311000 & 0.710940000 & 2.686581000 & & & & \\
\hline $\mathrm{H}$ & 1.027034000 & -0.051855000 & 2.785483000 & & & & \\
\hline C & 3.331336000 & 0.601715000 & -0.196759000 & & & & \\
\hline C & 4.715687000 & 0.341164000 & -0.272376000 & & & & \\
\hline H & 5.088319000 & -0.655093000 & -0.077420000 & & & & \\
\hline C & 5.637721000 & 1.332163000 & -0.594700000 & & & & \\
\hline $\mathrm{H}$ & 6.694825000 & 1.090608000 & -0.643063000 & & & & \\
\hline $\mathrm{H}$ & 5.893456000 & 3.412980000 & -1.108838000 & & & & \\
\hline C & 5.190369000 & 2.626062000 & -0.854089000 & & & & \\
\hline C & 3.829258000 & 2.905211000 & -0.782638000 & & & & \\
\hline $\mathrm{H}$ & 3.476282000 & 3.912668000 & -0.979581000 & & & & \\
\hline C & 2.879784000 & 1.919805000 & -0.456565000 & & & & \\
\hline C & 1.459564000 & 2.399878000 & -0.418573000 & & & & \\
\hline C & 0.743574000 & 2.575260000 & -1.614322000 & & & & \\
\hline $\mathrm{H}$ & 1.185199000 & 2.252463000 & -2.552594000 & & & & \\
\hline C & -0.511400000 & 3.187546000 & -1.609189000 & & & & \\
\hline $\mathrm{H}$ & -1.044502000 & 3.328905000 & -2.544641000 & & & & \\
\hline $\mathrm{H}$ & -2.024487000 & 4.151409000 & -0.412660000 & & & & \\
\hline C & -1.063942000 & 3.644591000 & -0.410781000 & & & & \\
\hline H & -0.773138000 & 3.849027000 & 1.718067000 & & & & \\
\hline C & -0.362819000 & 3.473082000 & 0.784951000 & & & & \\
\hline C & 0.890274000 & 2.854324000 & 0.781961000 & & & & \\
\hline $\mathrm{H}$ & 1.448351000 & 2.759225000 & 1.708426000 & & & & \\
\hline $\mathrm{H}$ & 3.452342000 & -0.783255000 & -2.603662000 & & & & \\
\hline \multicolumn{4}{|c|}{ TSe $\left(\mathrm{H}_{3} \mathrm{PAu}^{+}\right)$} & \multicolumn{4}{|c|}{ TSe $\left(\mathrm{Me}_{3} \mathrm{PAu}^{+}\right)$} \\
\hline C & -0.272161000 & -0.379746000 & 0.100694000 & C & -0.871739000 & -0.429279000 & 0.126783000 \\
\hline C & -1.168324000 & -2.005637000 & -0.753605000 & C & -1.794102000 & -1.995436000 & -0.740259000 \\
\hline C & -1.403854000 & 0.358802000 & 0.194616000 & C & -1.979152000 & 0.352188000 & 0.210993000 \\
\hline C & -2.702330000 & -0.247968000 & -0.088057000 & C & -3.293084000 & -0.206578000 & -0.096040000 \\
\hline C & -2.589854000 & -1.759248000 & -0.296858000 & C & -3.221611000 & -1.717862000 & -0.316970000 \\
\hline C & -2.930476000 & -2.567090000 & 0.971552000 & C & -3.613576000 & -2.525526000 & 0.936716000 \\
\hline C & -3.841759000 & 0.485922000 & -0.151172000 & C & -4.406826000 & 0.564259000 & -0.172650000 \\
\hline
\end{tabular}




\begin{tabular}{|c|c|c|c|c|c|c|c|}
\hline c & -5.196355000 & -0.103806000 & -0.419205000 & C & -5.775579000 & 0.020167000 & -0.465485000 \\
\hline c & -3.815638000 & 1.985832000 & 0.052324000 & C & -4.333914000 & 2.061017000 & 0.041824000 \\
\hline c & -1.355521000 & 1.818115000 & 0.581860000 & c & -1.886621000 & 1.807515000 & 0.601032000 \\
\hline C & -2.434975000 & 2.609729000 & -0.167849000 & C & -2.929848000 & 2.639207000 & -0.156767000 \\
\hline $\mathrm{Au}$ & 1.738861000 & -0.067046000 & 0.008662000 & $\mathrm{Au}$ & 1.165821000 & -0.164162000 & 0.037634000 \\
\hline $\mathrm{P}$ & 4.076367000 & 0.338044000 & -0.083291000 & $\mathrm{P}$ & 3.509049000 & 0.189133000 & -0.059152000 \\
\hline $\mathrm{H}$ & 4.881881000 & -0.711064000 & -0.562690000 & H & -0.873165000 & 2.184000000 & 0.439506000 \\
\hline $\mathrm{H}$ & 4.509575000 & 1.402975000 & -0.894089000 & $\mathrm{H}$ & -2.081449000 & 1.871723000 & 1.681431000 \\
\hline $\mathrm{H}$ & 4.721605000 & 0.641857000 & 1.129512000 & H & -2.684048000 & 2.644362000 & -1.225451000 \\
\hline $\mathrm{H}$ & -0.358130000 & 2.230385000 & 0.407848000 & $\mathrm{H}$ & -2.895290000 & 3.677998000 & 0.184462000 \\
\hline $\mathrm{H}$ & -1.540801000 & 1.877386000 & 1.663887000 & $\mathrm{H}$ & -5.047270000 & 2.554014000 & -0.628819000 \\
\hline $\mathrm{H}$ & -2.202334000 & 2.617748000 & -1.239271000 & $\mathrm{H}$ & -4.692286000 & 2.277662000 & 1.060354000 \\
\hline $\mathrm{H}$ & -2.430329000 & 3.650532000 & 0.168549000 & H & -6.184964000 & 0.487769000 & -1.369477000 \\
\hline H & -4.553664000 & 2.449563000 & -0.612298000 & H & -6.464561000 & 0.277097000 & 0.349286000 \\
\hline $\mathrm{H}$ & -4.168658000 & 2.196601000 & 1.073871000 & H & -5.801467000 & -1.062433000 & -0.595905000 \\
\hline $\mathrm{H}$ & -5.629624000 & 0.338375000 & -1.324838000 & $\mathrm{H}$ & -3.872750000 & -2.007777000 & -1.150272000 \\
\hline $\mathrm{H}$ & -5.882708000 & 0.145506000 & 0.399964000 & $\mathrm{H}$ & -4.641744000 & -2.304458000 & 1.231691000 \\
\hline $\mathrm{H}$ & -5.192085000 & -1.188367000 & -0.533754000 & $\mathrm{H}$ & -3.539253000 & -3.601570000 & 0.750936000 \\
\hline $\mathrm{H}$ & -3.249701000 & -2.073863000 & -1.114395000 & H & -2.968249000 & -2.276521000 & 1.786307000 \\
\hline $\mathrm{H}$ & -3.958349000 & -2.373955000 & 1.286218000 & $\mathrm{H}$ & -1.403616000 & -2.995143000 & -0.524160000 \\
\hline $\mathrm{H}$ & -2.828207000 & -3.641991000 & 0.792876000 & H & -1.077253000 & -1.577688000 & 0.328242000 \\
\hline $\mathrm{H}$ & -2.274974000 & -2.291365000 & 1.804823000 & $\mathrm{H}$ & -1.495581000 & -1.672181000 & -1.733586000 \\
\hline $\mathrm{H}$ & -0.746027000 & -2.993929000 & -0.544202000 & C & 4.433446000 & -1.216510000 & -0.797412000 \\
\hline $\mathrm{H}$ & -0.448873000 & -1.551698000 & 0.267064000 & $\mathrm{H}$ & 5.506407000 & -1.003186000 & -0.815146000 \\
\hline \multirow[t]{11}{*}{$\mathrm{H}$} & -0.896653000 & -1.668188000 & -1.749799000 & $\mathrm{H}$ & 4.256267000 & -2.121894000 & -0.211803000 \\
\hline & & & & $\mathrm{H}$ & 4.084326000 & -1.389784000 & -1.818285000 \\
\hline & & & & C & 4.280844000 & 0.457633000 & 1.586126000 \\
\hline & & & & $\mathrm{H}$ & 3.828104000 & 1.328024000 & 2.067318000 \\
\hline & & & & $\mathrm{H}$ & 4.106954000 & -0.416031000 & 2.219059000 \\
\hline & & & & $\mathrm{H}$ & 5.357870000 & 0.621314000 & 1.484306000 \\
\hline & & & & C & 3.991519000 & 1.652913000 & -1.058878000 \\
\hline & & & & H & 5.079142000 & 1.771782000 & -1.066018000 \\
\hline & & & & H & 3.635517000 & 1.530218000 & -2.084699000 \\
\hline & & & & $\mathrm{H}$ & 3.534209000 & 2.552236000 & -0.639145000 \\
\hline & \multicolumn{3}{|c|}{ TSe $\left(\mathrm{Et}_{3} \mathrm{PAu}^{+}\right)$} & \multicolumn{4}{|c|}{ TSe $\left(\mathrm{Ph}_{3} \mathrm{PAu}^{+}\right)$} \\
\hline C & 1.345471000 & -0.402344000 & -0.215223000 & C & 2.438073000 & -0.541622000 & -0.163516000 \\
\hline C & 2.262237000 & -2.130857000 & 0.228150000 & C & 3.388728000 & -2.068613000 & 0.724038000 \\
\hline C & 2.461487000 & 0.368760000 & -0.136037000 & C & 3.524178000 & 0.270721000 & -0.242778000 \\
\hline C & 3.774185000 & -0.258065000 & -0.006075000 & c & 4.849424000 & -0.248523000 & 0.085195000 \\
\hline C & 3.684217000 & -1.777001000 & -0.155948000 & C & 4.814934000 & -1.759042000 & 0.317979000 \\
\hline C & 4.029167000 & -2.261990000 & -1.578414000 & C & 5.244611000 & -2.565613000 & -0.924004000 \\
\hline C & 4.899783000 & 0.461682000 & 0.228352000 & C & 5.940140000 & 0.553097000 & 0.171210000 \\
\hline C & 6.267308000 & -0.147834000 & 0.345326000 & C & 7.319428000 & 0.049119000 & 0.486639000 \\
\hline C & 4.842163000 & 1.965725000 & 0.391244000 & C & 5.828353000 & 2.045857000 & -0.054664000 \\
\hline C & 2.380524000 & 1.875624000 & -0.156427000 & C & 3.395902000 & 1.720225000 & -0.643422000 \\
\hline C & 3.450066000 & 2.488317000 & 0.757455000 & C & 4.405939000 & 2.585532000 & 0.121806000 \\
\hline
\end{tabular}




\begin{tabular}{|c|c|c|c|c|c|c|c|}
\hline $\mathrm{Au}$ & -0.691771000 & -0.147377000 & -0.028549000 & $\mathrm{Au}$ & 0.393495000 & -0.317674000 & -0.081708000 \\
\hline $\mathrm{H}$ & 1.375643000 & 2.208342000 & 0.116371000 & $\mathrm{H}$ & 2.370247000 & 2.068920000 & -0.497939000 \\
\hline $\mathrm{H}$ & 2.553734000 & 2.201823000 & -1.192355000 & $\mathrm{H}$ & 3.602696000 & 1.783465000 & -1.721719000 \\
\hline $\mathrm{H}$ & 3.226113000 & 2.233308000 & 1.800275000 & $\mathrm{H}$ & 4.146497000 & 2.589991000 & 1.187368000 \\
\hline $\mathrm{H}$ & 3.422269000 & 3.579351000 & 0.682299000 & $\mathrm{H}$ & 4.346858000 & 3.621024000 & -0.226251000 \\
\hline $\mathrm{H}$ & 5.574823000 & 2.271919000 & 1.147104000 & $\mathrm{H}$ & 6.518724000 & 2.563070000 & 0.621937000 \\
\hline $\mathrm{H}$ & 5.184072000 & 2.424673000 & -0.549654000 & H & 6.193692000 & 2.266427000 & -1.069885000 \\
\hline $\mathrm{H}$ & 6.697232000 & 0.070574000 & 1.330748000 & $\mathrm{H}$ & 7.703845000 & 0.534364000 & 1.392332000 \\
\hline $\mathrm{H}$ & 6.945060000 & 0.304431000 & -0.389917000 & H & 8.012464000 & 0.317992000 & -0.320869000 \\
\hline $\mathrm{H}$ & 6.283315000 & -1.228015000 & 0.194151000 & $\mathrm{H}$ & 7.372695000 & -1.031467000 & 0.625977000 \\
\hline $\mathrm{H}$ & 4.351080000 & -2.267425000 & 0.563155000 & $\mathrm{H}$ & 5.462928000 & -2.025399000 & 1.161501000 \\
\hline $\mathrm{H}$ & 5.054539000 & -1.991939000 & -1.840931000 & $\mathrm{H}$ & 6.270589000 & -2.320029000 & -1.207263000 \\
\hline $\mathrm{H}$ & 3.936583000 & -3.349778000 & -1.656551000 & H & 5.195769000 & -3.641864000 & -0.730930000 \\
\hline H & 3.368915000 & -1.805246000 & -2.323907000 & H & 4.604283000 & -2.340038000 & -1.783838000 \\
\hline $\mathrm{H}$ & 1.848800000 & -3.045563000 & -0.208696000 & H & 3.026458000 & -3.079450000 & 0.510606000 \\
\hline $\mathrm{H}$ & 1.526300000 & -1.463178000 & -0.700029000 & $\mathrm{H}$ & 2.669885000 & -1.681813000 & -0.363552000 \\
\hline $\mathrm{H}$ & 1.995117000 & -2.052403000 & 1.278328000 & $\mathrm{H}$ & 3.069928000 & -1.747753000 & 1.711936000 \\
\hline$P$ & -3.033283000 & 0.181146000 & 0.183997000 & $P$ & -1.959157000 & 0.020706000 & 0.011665000 \\
\hline C & -3.516738000 & 1.964771000 & 0.281544000 & c & -2.368252000 & 1.805888000 & 0.006088000 \\
\hline $\mathrm{H}$ & -3.053517000 & 2.356175000 & 1.194054000 & C & -1.591949000 & 2.674345000 & 0.791617000 \\
\hline $\mathrm{H}$ & -4.601467000 & 2.014914000 & 0.429389000 & H & -0.759990000 & 2.283904000 & 1.372241000 \\
\hline C & -3.090838000 & 2.794741000 & -0.936393000 & $\mathrm{H}$ & -1.286427000 & 4.700385000 & 1.445617000 \\
\hline $\mathrm{H}$ & -3.577991000 & 2.451984000 & -1.853914000 & C & -1.888484000 & 4.035483000 & 0.833861000 \\
\hline $\mathrm{H}$ & -2.008242000 & 2.750896000 & -1.091724000 & C & -2.954667000 & 4.542512000 & 0.086139000 \\
\hline $\mathrm{H}$ & -3.366862000 & 3.842653000 & -0.790035000 & $\mathrm{H}$ & -3.181295000 & 5.603912000 & 0.114861000 \\
\hline C & -3.744880000 & -0.576422000 & 1.715221000 & $\mathrm{H}$ & -4.552522000 & 4.077350000 & -1.284291000 \\
\hline $\mathrm{H}$ & -3.282139000 & -0.049860000 & 2.557271000 & C & -3.725550000 & 3.685355000 & -0.700240000 \\
\hline $\mathrm{H}$ & -4.815337000 & -0.343665000 & 1.744229000 & C & -3.437039000 & 2.319707000 & -0.742452000 \\
\hline $\mathrm{H}$ & -3.994652000 & -2.641030000 & 1.023373000 & $\mathrm{H}$ & -4.039354000 & 1.660925000 & -1.359347000 \\
\hline C & -3.508927000 & -2.087499000 & 1.832485000 & C & -2.711255000 & -0.681356000 & 1.526113000 \\
\hline H & -2.440800000 & -2.326156000 & 1.813611000 & C & -3.786038000 & -0.051977000 & 2.172020000 \\
\hline $\mathrm{H}$ & -3.660136000 & -1.601666000 & -1.294959000 & H & -4.174159000 & 0.888899000 & 1.795376000 \\
\hline C & -3.949212000 & -0.545760000 & -1.253864000 & C & -4.354553000 & -0.632128000 & 3.307210000 \\
\hline $\mathrm{H}$ & -3.520267000 & -0.083905000 & -2.149904000 & H & -5.184237000 & -0.138982000 & 3.804280000 \\
\hline $\mathrm{H}$ & -3.918580000 & -2.458451000 & 2.776039000 & C & -3.856833000 & -1.838252000 & 3.803504000 \\
\hline $\mathrm{H}$ & -5.927856000 & -0.886702000 & -0.370537000 & $\mathrm{H}$ & -4.299728000 & -2.284937000 & 4.688412000 \\
\hline C & -5.476021000 & -0.395940000 & -1.237220000 & $\mathrm{H}$ & -2.394705000 & -3.404341000 & 3.551881000 \\
\hline $\mathrm{H}$ & -5.900815000 & -0.858943000 & -2.132586000 & C & -2.785566000 & -2.468123000 & 3.165290000 \\
\hline \multirow[t]{8}{*}{$\mathrm{H}$} & -5.785772000 & 0.653042000 & -1.234382000 & C & -2.211263000 & -1.891428000 & 2.033470000 \\
\hline & & & & $\mathrm{H}$ & -1.374125000 & -2.380515000 & 1.542419000 \\
\hline & & & & C & -2.831821000 & -0.730365000 & -1.411721000 \\
\hline & & & & C & -2.214548000 & -0.705427000 & -2.673239000 \\
\hline & & & & $\mathrm{H}$ & -1.226785000 & -0.266647000 & -2.786680000 \\
\hline & & & & $\mathrm{H}$ & -2.382190000 & -1.220080000 & -4.754718000 \\
\hline & & & & C & -2.864985000 & -1.243580000 & -3.782616000 \\
\hline & & & & C & -4.130164000 & -1.818876000 & -3.640548000 \\
\hline
\end{tabular}




\begin{tabular}{|c|c|c|c|c|c|c|c|}
\hline & & & & $\mathrm{H}$ & -4.632799000 & -2.244076000 & -4.503871000 \\
\hline & & & & $\mathrm{H}$ & -5.726829000 & -2.303066000 & -2.274644000 \\
\hline & & & & c & -4.745655000 & -1.852440000 & -2.388134000 \\
\hline & & & & C & -4.102034000 & -1.310372000 & -1.274408000 \\
\hline & & & & H & -4.585495000 & -1.345119000 & -0.303598000 \\
\hline & & $\underline{I S e}\left(t \mathbf{B u}_{3} \mathbf{P A u}^{+}\right)$ & & & & TSe $\left(\operatorname{IPrAu}{ }^{+}\right)$ & \\
\hline C & 1.996970000 & -0.492741000 & -0.156672000 & C & 2.312995000 & -0.880806000 & -0.343473000 \\
\hline c & 2.943750000 & -2.019075000 & 0.722498000 & c & 2.888348000 & -2.739664000 & 0.030761000 \\
\hline C & 3.084651000 & 0.320321000 & -0.231162000 & C & 3.549567000 & -0.321873000 & -0.234466000 \\
\hline C & 4.409172000 & -0.200766000 & 0.095981000 & c & 4.726651000 & -1.179077000 & -0.123121000 \\
\hline c & 4.371789000 & -1.712208000 & 0.320561000 & c & 4.360320000 & -2.649896000 & -0.320991000 \\
\hline C & 4.801065000 & -2.512726000 & -0.925508000 & C & 4.637139000 & -3.147684000 & -1.753973000 \\
\hline c & 5.500883000 & 0.599129000 & 0.187233000 & c & 5.961981000 & -0.684309000 & 0.139150000 \\
\hline C & 6.879510000 & 0.091665000 & 0.499821000 & C & 7.194832000 & -1.536259000 & 0.239879000 \\
\hline C & 5.390790000 & 2.093087000 & -0.030954000 & C & 6.175342000 & 0.800121000 & 0.348103000 \\
\hline C & 2.959056000 & 1.772004000 & -0.623820000 & C & 3.738556000 & 1.173867000 & -0.195000000 \\
\hline c & 3.968652000 & 2.633062000 & 0.146748000 & C & 4.897921000 & 1.550370000 & 0.736865000 \\
\hline $\mathrm{Au}$ & -0.054325000 & -0.261510000 & -0.073170000 & $\mathrm{Au}$ & 0.358919000 & -0.283352000 & -0.136778000 \\
\hline H & 1.933397000 & 2.119946000 & -0.477159000 & H & 2.807778000 & 1.669521000 & 0.091594000 \\
\hline H & 3.167607000 & 1.841381000 & -1.701601000 & H & 3.971481000 & 1.503648000 & -1.218325000 \\
\hline $\mathrm{H}$ & 3.708107000 & 2.632370000 & 1.212162000 & $\mathrm{H}$ & 4.628641000 & 1.299985000 & 1.770204000 \\
\hline $\mathrm{H}$ & 3.910966000 & 3.670492000 & -0.195800000 & $\mathrm{H}$ & 5.067133000 & 2.630803000 & 0.703386000 \\
\hline H & 6.080945000 & 2.606448000 & 0.648774000 & H & 6.950314000 & 0.947229000 & 1.109634000 \\
\hline H & 5.757098000 & 2.318634000 & -1.044773000 & H & 6.593482000 & 1.220961000 & -0.579970000 \\
\hline $\mathrm{H}$ & 7.266445000 & 0.574163000 & 1.405884000 & $\mathrm{H}$ & 7.669821000 & -1.405712000 & 1.220089000 \\
\hline $\mathrm{H}$ & 7.572060000 & 0.360456000 & -0.308185000 & H & 7.936569000 & -1.216622000 & -0.503555000 \\
\hline $\mathrm{H}$ & 6.930372000 & -0.989336000 & 0.636849000 & $\mathrm{H}$ & 7.007238000 & -2.600030000 & 0.087590000 \\
\hline $\mathrm{H}$ & 5.018623000 & -1.985178000 & 1.162849000 & H & 4.912340000 & -3.277220000 & 0.388825000 \\
\hline $\mathrm{H}$ & 5.828103000 & -2.268388000 & -1.206026000 & $\mathrm{H}$ & 5.699745000 & -3.065183000 & -1.993763000 \\
\hline $\mathrm{H}$ & 4.749096000 & -3.589943000 & -0.738739000 & $\mathrm{H}$ & 4.345274000 & -4.196360000 & -1.869465000 \\
\hline $\mathrm{H}$ & 4.162745000 & -2.280560000 & -1.785216000 & $\mathrm{H}$ & 4.087385000 & -2.553917000 & -2.492786000 \\
\hline $\mathrm{H}$ & 2.579588000 & -3.027578000 & 0.501695000 & $\mathrm{H}$ & 2.320579000 & -3.542991000 & -0.449374000 \\
\hline $\mathrm{H}$ & 2.223579000 & -1.626305000 & -0.374454000 & $\mathrm{H}$ & 2.307683000 & -1.914848000 & -0.900543000 \\
\hline H & 2.626079000 & -1.706400000 & 1.713473000 & $\mathrm{H}$ & 2.622070000 & -2.665235000 & 1.081593000 \\
\hline$P$ & -2.428140000 & 0.075401000 & 0.028115000 & C & -1.583320000 & 0.344345000 & 0.073774000 \\
\hline C & -2.950316000 & 1.170616000 & -1.482821000 & N & -2.040748000 & 1.624716000 & 0.096280000 \\
\hline C & -2.497693000 & 2.627968000 & -1.243245000 & C & -3.420219000 & 1.650208000 & 0.258796000 \\
\hline $\mathrm{H}$ & -1.429606000 & 2.693011000 & -1.014715000 & $\mathrm{H}$ & -3.971914000 & 2.575490000 & 0.300033000 \\
\hline C & -4.249202000 & 1.594449000 & 1.738148000 & C & -3.829137000 & 0.358003000 & 0.339328000 \\
\hline H & -4.414344000 & 2.017516000 & 2.735653000 & H & -4.809773000 & -0.072114000 & 0.464133000 \\
\hline $\mathrm{H}$ & -3.059375000 & 3.129431000 & -0.454248000 & C & -0.695065000 & 3.394259000 & 1.140457000 \\
\hline $\mathrm{H}$ & -4.365799000 & 2.410350000 & 1.022639000 & C & -1.217573000 & 2.810385000 & -0.031026000 \\
\hline $\mathrm{H}$ & -2.668549000 & 3.191906000 & -2.166783000 & C & -0.998310000 & 3.342559000 & -1.317492000 \\
\hline H & -5.041993000 & 0.865896000 & 1.559270000 & C & -1.569520000 & 2.718153000 & -2.587159000 \\
\hline C & -4.466313000 & 1.158669000 & -1.767431000 & C & -0.449360000 & 2.254360000 & -3.538436000 \\
\hline $\mathrm{H}$ & -5.060177000 & 1.497825000 & -0.916373000 & $\mathrm{H}$ & 0.220837000 & 1.544702000 & -3.042406000 \\
\hline
\end{tabular}




\begin{tabular}{|c|c|c|c|c|c|c|c|}
\hline $\mathrm{H}$ & -4.669928000 & 1.842824000 & -2.599183000 & $\mathrm{H}$ & -0.877598000 & 1.762895000 & -4.418167000 \\
\hline $\mathrm{H}$ & -4.828559000 & 0.174298000 & -2.069158000 & $\mathrm{H}$ & 0.152994000 & 3.097659000 & -3.891661000 \\
\hline $\mathrm{H}$ & -5.375192000 & -0.951310000 & -0.208539000 & $\mathrm{H}$ & -2.140094000 & 1.828360000 & -2.305362000 \\
\hline C & -4.768837000 & -1.640047000 & 0.382517000 & $\mathrm{H}$ & -2.976858000 & 3.195643000 & -4.180328000 \\
\hline c & -2.187225000 & 0.689022000 & -2.740516000 & C & -2.542711000 & 3.678617000 & -3.299042000 \\
\hline $\mathrm{H}$ & -2.437760000 & 1.361352000 & -3.568952000 & $\mathrm{H}$ & -3.362586000 & 3.981166000 & -2.639931000 \\
\hline H & -2.447557000 & -0.320349000 & -3.053007000 & $\mathrm{H}$ & -2.035127000 & 4.588054000 & -3.636204000 \\
\hline $\mathrm{H}$ & -3.826641000 & -1.679216000 & -2.198538000 & $\mathrm{H}$ & -0.028315000 & 4.942373000 & -2.379593000 \\
\hline $\mathrm{H}$ & -3.536317000 & -3.254225000 & -1.475257000 & C & -0.216558000 & 4.501338000 & -1.405741000 \\
\hline C & -3.193646000 & -2.213728000 & -1.489198000 & C & 0.317653000 & 5.099922000 & -0.268102000 \\
\hline $\mathrm{H}$ & -2.164804000 & -2.213582000 & -1.861706000 & $\mathrm{H}$ & 0.916365000 & 6.001238000 & -0.361084000 \\
\hline $\mathrm{H}$ & -2.972968000 & -3.636530000 & 0.749276000 & C & 0.080149000 & 4.550773000 & 0.988994000 \\
\hline C & -2.505696000 & -2.649596000 & 0.843244000 & $\mathrm{H}$ & 0.495761000 & 5.030558000 & 1.869656000 \\
\hline H & -1.464769000 & -2.744445000 & 0.519145000 & $\mathrm{H}$ & -2.683913000 & 4.098066000 & 2.953567000 \\
\hline $\mathrm{H}$ & -2.511643000 & -2.386144000 & 1.899148000 & C & -1.729226000 & 3.824452000 & 3.413656000 \\
\hline $\mathrm{H}$ & -1.103775000 & 0.732593000 & -2.593983000 & $\mathrm{H}$ & -1.163965000 & 4.747371000 & 3.578399000 \\
\hline c & -3.288728000 & -1.658458000 & -0.051381000 & $\mathrm{H}$ & -1.939084000 & 3.384296000 & 4.393785000 \\
\hline $\mathrm{H}$ & -4.894612000 & -1.388652000 & 1.437397000 & C & 0.372401000 & 2.406983000 & 3.216573000 \\
\hline H & -5.185934000 & -2.643797000 & 0.241862000 & $\mathrm{H}$ & 0.907066000 & 1.663792000 & 2.615728000 \\
\hline C & -2.840367000 & 0.967504000 & 1.697624000 & $\mathrm{H}$ & 1.038337000 & 3.262775000 & 3.367866000 \\
\hline C & -2.705146000 & -0.032275000 & 2.867075000 & $\mathrm{H}$ & 0.168570000 & 1.968855000 & 4.199083000 \\
\hline $\mathrm{H}$ & -2.788433000 & 0.529026000 & 3.804310000 & C & -0.944314000 & 2.829368000 & 2.536089000 \\
\hline $\mathrm{H}$ & -1.731232000 & -0.531012000 & 2.871405000 & $\mathrm{H}$ & -1.558039000 & 1.929277000 & 2.436862000 \\
\hline $\mathrm{H}$ & -3.490869000 & -0.788696000 & 2.877008000 & $\mathrm{~N}$ & -2.691003000 & -0.430172000 & 0.224298000 \\
\hline C & -1.782056000 & 2.068580000 & 1.949870000 & C & -2.698033000 & -1.878643000 & 0.256416000 \\
\hline H & -1.786868000 & 2.858560000 & 1.201405000 & C & -2.870495000 & -2.576433000 & -0.955885000 \\
\hline $\mathrm{H}$ & -1.997595000 & 2.534335000 & 2.918315000 & C & -2.554823000 & -2.524780000 & 1.500703000 \\
\hline \multirow[t]{19}{*}{$\mathrm{H}$} & -0.772123000 & 1.650354000 & 2.000893000 & C & -2.394025000 & -1.770363000 & 2.817120000 \\
\hline & & & & $\mathrm{H}$ & -2.405789000 & -0.698022000 & 2.601414000 \\
\hline & & & & $\mathrm{H}$ & -0.207342000 & -1.832338000 & 2.819469000 \\
\hline & & & & C & -1.040530000 & -2.081549000 & 3.485014000 \\
\hline & & & & $\mathrm{H}$ & -0.957582000 & -3.140274000 & 3.751862000 \\
\hline & & & & $\mathrm{H}$ & -0.928345000 & -1.498629000 & 4.405015000 \\
\hline & & & & $\mathrm{H}$ & -3.459351000 & -1.468478000 & 4.693441000 \\
\hline & & & & C & -3.566311000 & -2.056594000 & 3.776358000 \\
\hline & & & & $\mathrm{H}$ & -4.528272000 & -1.802157000 & 3.320494000 \\
\hline & & & & $\mathrm{H}$ & -3.603443000 & -3.112711000 & 4.062112000 \\
\hline & & & & $\mathrm{H}$ & -2.469085000 & -4.458145000 & 2.440517000 \\
\hline & & & & C & -2.574147000 & -3.925143000 & 1.500633000 \\
\hline & & & & $\mathrm{H}$ & -2.751232000 & -5.729793000 & 0.344254000 \\
\hline & & & & C & -2.733719000 & -4.644188000 & 0.319153000 \\
\hline & & & & $\mathrm{H}$ & -3.015315000 & -4.547308000 & -1.806004000 \\
\hline & & & & C & -2.881812000 & -3.975527000 & -0.892989000 \\
\hline & & & & $\mathrm{H}$ & -3.015908000 & -0.797142000 & -2.132923000 \\
\hline & & & & C & -3.049224000 & -1.877786000 & -2.300515000 \\
\hline & & & & C & -4.421744000 & -2.199519000 & -2.924666000 \\
\hline
\end{tabular}




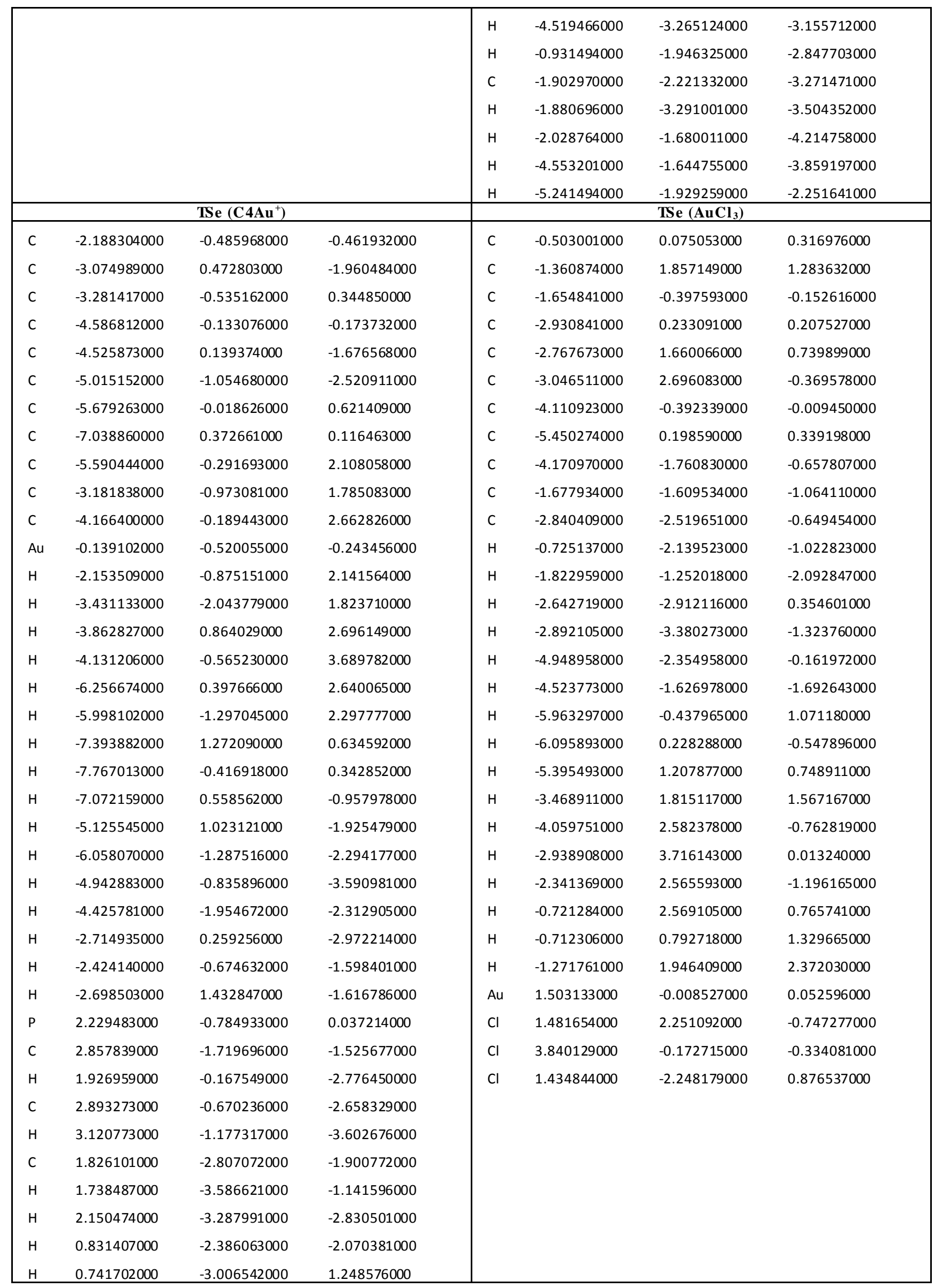




\begin{tabular}{|c|c|c|c|c|c|c|c|}
\hline c & 1.773997000 & -3.093623000 & 1.602313000 & & & & \\
\hline $\mathrm{H}$ & 2.305904000 & -3.793028000 & 0.951719000 & & & & \\
\hline $\mathrm{H}$ & 1.747391000 & -3.540662000 & 2.602463000 & & & & \\
\hline $\mathrm{H}$ & 4.254756000 & -3.174488000 & -0.640340000 & & & & \\
\hline c & 4.238647000 & -2.391697000 & -1.402754000 & & & & \\
\hline $\mathrm{H}$ & 4.473571000 & -2.871595000 & -2.359694000 & & & & \\
\hline $\mathrm{H}$ & 5.043974000 & -1.683957000 & -1.200081000 & & & & \\
\hline $\mathrm{H}$ & 4.530979000 & -2.505561000 & 1.394235000 & & & & \\
\hline C & 3.945122000 & -1.944198000 & 2.124993000 & & & & \\
\hline $\mathrm{H}$ & 3.941909000 & -2.527702000 & 3.052785000 & & & & \\
\hline $\mathrm{H}$ & 4.457371000 & -1.004293000 & 2.339164000 & & & & \\
\hline c & 2.482829000 & -1.725575000 & 1.690191000 & & & & \\
\hline C & 1.787280000 & -0.862595000 & 2.766907000 & & & & \\
\hline $\mathrm{H}$ & 1.883876000 & -1.361306000 & 3.737880000 & & & & \\
\hline $\mathrm{H}$ & 2.247995000 & 0.125735000 & 2.855483000 & & & & \\
\hline $\mathrm{H}$ & 0.720967000 & -0.729366000 & 2.560571000 & & & & \\
\hline C & 3.239574000 & 0.763692000 & 0.166897000 & & & & \\
\hline C & 4.640526000 & 0.627753000 & 0.262681000 & & & & \\
\hline H & 5.083254000 & -0.357782000 & 0.285980000 & & & & \\
\hline C & 5.490795000 & 1.727214000 & 0.330890000 & & & & \\
\hline $\mathrm{H}$ & 6.563316000 & 1.576481000 & 0.403516000 & & & & \\
\hline $\mathrm{H}$ & 5.597152000 & 3.883414000 & 0.354264000 & & & & \\
\hline C & 4.952246000 & 3.011592000 & 0.303224000 & & & & \\
\hline C & 3.573332000 & 3.169908000 & 0.210199000 & & & & \\
\hline $\mathrm{H}$ & 3.148179000 & 4.168550000 & 0.191805000 & & & & \\
\hline C & 2.695462000 & 2.072162000 & 0.142293000 & & & & \\
\hline C & 1.244360000 & 2.443683000 & 0.049326000 & & & & \\
\hline C & 0.704249000 & 2.851908000 & -1.180467000 & & & & \\
\hline $\mathrm{H}$ & 1.310570000 & 2.779215000 & -2.078722000 & & & & \\
\hline C & -0.583408000 & 3.389481000 & -1.249633000 & & & & \\
\hline $\mathrm{H}$ & -0.972483000 & 3.730117000 & -2.205325000 & & & & \\
\hline $\mathrm{H}$ & -2.336164000 & 3.980049000 & -0.135967000 & & & & \\
\hline C & -1.348801000 & 3.529187000 & -0.089020000 & & & & \\
\hline H & -1.402781000 & 3.245860000 & 2.048968000 & & & & \\
\hline C & -0.823052000 & 3.120090000 & 1.139104000 & & & & \\
\hline C & 0.464372000 & 2.584497000 & 1.208377000 & & & & \\
\hline $\mathrm{H}$ & 0.882323000 & 2.304962000 & 2.170698000 & & & & \\
\hline $\mathrm{H}$ & 3.658992000 & 0.091569000 & -2.493986000 & & & & \\
\hline \multicolumn{4}{|c|}{ Int6 $\left(\mathrm{H}_{3} \mathrm{PAu}^{+}\right)$} & \multicolumn{4}{|c|}{ Int6 $\left(\mathrm{Me}_{3} \mathrm{PAu}^{+}\right)$} \\
\hline C & -0.303718000 & 0.797292000 & -1.372057000 & C & 1.032858000 & -1.026180000 & -1.384344000 \\
\hline C & -0.853706000 & 2.111746000 & -0.818941000 & C & 1.563774000 & -2.215086000 & -0.589993000 \\
\hline C & -1.162784000 & -0.246200000 & -0.966689000 & C & 1.721588000 & 0.129049000 & -1.002154000 \\
\hline C & -2.067191000 & 0.198240000 & 0.068353000 & C & 2.509142000 & -0.107091000 & 0.196339000 \\
\hline C & -1.843201000 & 1.698692000 & 0.315202000 & C & 2.402829000 & -1.592891000 & 0.573013000 \\
\hline C & -3.098909000 & 2.585606000 & 0.288135000 & C & 3.743422000 & -2.330896000 & 0.728153000 \\
\hline C & -2.922297000 & -0.680978000 & 0.671585000 & C & 3.177583000 & 0.909127000 & 0.807717000 \\
\hline
\end{tabular}




\begin{tabular}{|c|c|c|c|c|c|c|c|}
\hline c & -3.866423000 & -0.302563000 & 1.773280000 & C & 3.982139000 & 0.744356000 & 2.064369000 \\
\hline c & -2.983115000 & -2.127333000 & 0.223774000 & C & 3.167073000 & 2.306105000 & 0.216716000 \\
\hline c & -1.261562000 & -1.618615000 & -1.553173000 & c & 1.799415000 & 1.437877000 & -1.726497000 \\
\hline C & -1.718655000 & -2.623479000 & -0.489014000 & C & 1.990727000 & 2.586758000 & -0.727848000 \\
\hline $\mathrm{Au}$ & 1.375295000 & 0.103950000 & -0.118786000 & $\mathrm{Au}$ & -0.828052000 & -0.252034000 & -0.367200000 \\
\hline $\mathrm{P}$ & 3.321904000 & -0.420209000 & 1.120816000 & $\mathrm{P}$ & -2.929666000 & 0.238980000 & 0.577776000 \\
\hline $\mathrm{H}$ & 4.146151000 & 0.667282000 & 1.461722000 & H & 0.929890000 & 1.591294000 & -2.372266000 \\
\hline $\mathrm{H}$ & 3.144138000 & -1.039358000 & 2.371641000 & $\mathrm{H}$ & 2.676810000 & 1.378165000 & -2.388972000 \\
\hline $\mathrm{H}$ & 4.241281000 & -1.288782000 & 0.505412000 & H & 1.072391000 & 2.703853000 & -0.138263000 \\
\hline $\mathrm{H}$ & -0.325399000 & -1.917363000 & -2.033485000 & $\mathrm{H}$ & 2.150463000 & 3.528777000 & -1.260099000 \\
\hline $\mathrm{H}$ & -2.017295000 & -1.558821000 & -2.352759000 & $\mathrm{H}$ & 3.179188000 & 3.041271000 & 1.029219000 \\
\hline $\mathrm{H}$ & -0.915452000 & -2.756213000 & 0.246937000 & $\mathrm{H}$ & 4.119776000 & 2.450752000 & -0.317353000 \\
\hline $\mathrm{H}$ & -1.900082000 & -3.601309000 & -0.943396000 & H & 3.774798000 & 1.568637000 & 2.755765000 \\
\hline H & -3.204090000 & -2.763057000 & 1.087880000 & H & 5.057437000 & 0.790693000 & 1.845043000 \\
\hline $\mathrm{H}$ & -3.855409000 & -2.230689000 & -0.441810000 & H & 3.784049000 & -0.195781000 & 2.579164000 \\
\hline $\mathrm{H}$ & -3.884470000 & -1.083877000 & 2.540266000 & $\mathrm{H}$ & 1.840202000 & -1.683447000 & 1.512788000 \\
\hline $\mathrm{H}$ & -4.892762000 & -0.225377000 & 1.388633000 & $\mathrm{H}$ & 4.328563000 & -1.976023000 & 1.578655000 \\
\hline $\mathrm{H}$ & -3.613728000 & 0.645617000 & 2.246968000 & $\mathrm{H}$ & 3.564944000 & -3.400718000 & 0.876399000 \\
\hline $\mathrm{H}$ & -1.343942000 & 1.822487000 & 1.287357000 & $\mathrm{H}$ & 4.354880000 & -2.214840000 & -0.172774000 \\
\hline $\mathrm{H}$ & -3.769846000 & 2.403658000 & 1.129224000 & $\mathrm{H}$ & 2.212429000 & -2.808106000 & -1.248967000 \\
\hline $\mathrm{H}$ & -2.806831000 & 3.639712000 & 0.327302000 & H & 0.648117000 & -1.146947000 & -2.396969000 \\
\hline $\mathrm{H}$ & -3.664583000 & 2.429721000 & -0.636264000 & $\mathrm{H}$ & 0.787129000 & -2.895682000 & -0.231400000 \\
\hline $\mathrm{H}$ & -1.400162000 & 2.619721000 & -1.625167000 & C & -4.020839000 & -1.233875000 & 0.658127000 \\
\hline $\mathrm{H}$ & 0.164072000 & 0.762071000 & -2.357576000 & $\mathrm{H}$ & -4.989273000 & -0.966713000 & 1.091668000 \\
\hline \multirow[t]{11}{*}{$\mathrm{H}$} & -0.089080000 & 2.811250000 & -0.473768000 & $\mathrm{H}$ & -4.175126000 & -1.632681000 & -0.347504000 \\
\hline & & & & $\mathrm{H}$ & -3.553048000 & -2.007897000 & 1.271291000 \\
\hline & & & & C & -3.871361000 & 1.497946000 & -0.368747000 \\
\hline & & & & $\mathrm{H}$ & -3.311745000 & 2.436002000 & -0.396350000 \\
\hline & & & & $\mathrm{H}$ & -4.021261000 & 1.151533000 & -1.394199000 \\
\hline & & & & $\mathrm{H}$ & -4.845135000 & 1.673357000 & 0.098583000 \\
\hline & & & & C & -2.832105000 & 0.879995000 & 2.295031000 \\
\hline & & & & H & -3.834733000 & 1.087432000 & 2.681136000 \\
\hline & & & & H & -2.344353000 & 0.141976000 & 2.936354000 \\
\hline & & & & $\mathrm{H}$ & -2.242800000 & 1.799896000 & 2.311930000 \\
\hline & \multicolumn{3}{|c|}{ Int6 $\left(\mathrm{Et}_{3} \mathrm{PAu}^{+}\right)$} & \multicolumn{4}{|c|}{ Int6 $\left(\mathrm{Ph}_{3} \mathrm{PAu}^{+}\right)$} \\
\hline C & 1.501767000 & -0.891727000 & -1.502790000 & C & 2.499730000 & -1.074091000 & -1.667015000 \\
\hline C & 2.011720000 & -2.154355000 & -0.817336000 & C & 3.029097000 & -2.278855000 & -0.899884000 \\
\hline C & 2.167930000 & 0.220870000 & -0.987359000 & C & 3.082872000 & 0.091442000 & -1.169842000 \\
\hline C & 2.935322000 & -0.133536000 & 0.197201000 & c & 3.802917000 & -0.174660000 & 0.068670000 \\
\hline C & 2.845087000 & -1.652651000 & 0.407086000 & C & 3.763480000 & -1.685126000 & 0.346174000 \\
\hline C & 4.197669000 & -2.382177000 & 0.475056000 & C & 5.136566000 & -2.352057000 & 0.535745000 \\
\hline C & 3.582244000 & 0.815466000 & 0.926100000 & C & 4.366405000 & 0.834363000 & 0.784525000 \\
\hline C & 4.363384000 & 0.524468000 & 2.175156000 & C & 5.101473000 & 0.631713000 & 2.079111000 \\
\hline C & 3.569993000 & 2.267591000 & 0.487463000 & C & 4.305530000 & 2.265196000 & 0.282989000 \\
\hline C & 2.250672000 & 1.600544000 & -1.566927000 & C & 3.135884000 & 1.448187000 & -1.805550000 \\
\hline C & 2.410696000 & 2.637461000 & -0.447433000 & C & 3.180216000 & 2.536622000 & -0.724611000 \\
\hline
\end{tabular}




\begin{tabular}{|c|c|c|c|c|c|c|c|}
\hline $\mathrm{Au}$ & -0.394977000 & -0.225805000 & -0.447745000 & $\mathrm{Au}$ & 0.552579000 & -0.395941000 & -0.709748000 \\
\hline $\mathrm{H}$ & 1.393389000 & 1.817603000 & -2.210893000 & $\mathrm{H}$ & 2.309045000 & 1.596134000 & -2.506257000 \\
\hline $\mathrm{H}$ & 3.142237000 & 1.618476000 & -2.212556000 & $\mathrm{H}$ & 4.062642000 & 1.483072000 & -2.398840000 \\
\hline $\mathrm{H}$ & 1.479779000 & 2.683505000 & 0.132389000 & $\mathrm{H}$ & 2.216795000 & 2.557987000 & -0.199186000 \\
\hline $\mathrm{H}$ & 2.571244000 & 3.632317000 & -0.872746000 & $\mathrm{H}$ & 3.314843000 & 3.520226000 & -1.183977000 \\
\hline $\mathrm{H}$ & 3.556956000 & 2.912397000 & 1.373549000 & $\mathrm{H}$ & 4.212231000 & 2.943197000 & 1.139111000 \\
\hline $\mathrm{H}$ & 4.531817000 & 2.476411000 & -0.007460000 & H & 5.280500000 & 2.505398000 & -0.170268000 \\
\hline $\mathrm{H}$ & 4.110178000 & 1.251906000 & 2.954796000 & $\mathrm{H}$ & 4.814468000 & 1.402899000 & 2.802568000 \\
\hline $\mathrm{H}$ & 5.441266000 & 0.632293000 & 1.993786000 & H & 6.184997000 & 0.735591000 & 1.931917000 \\
\hline $\mathrm{H}$ & 4.186078000 & -0.476615000 & 2.568285000 & $\mathrm{H}$ & 4.916084000 & -0.345245000 & 2.526263000 \\
\hline $\mathrm{H}$ & 2.289000000 & -1.855696000 & 1.332548000 & $\mathrm{H}$ & 3.156541000 & -1.867841000 & 1.243780000 \\
\hline $\mathrm{H}$ & 4.786608000 & -2.104012000 & 1.351232000 & $\mathrm{H}$ & 5.651372000 & -2.012930000 & 1.436664000 \\
\hline $\mathrm{H}$ & 4.037264000 & -3.464336000 & 0.516700000 & H & 5.017284000 & -3.437505000 & 0.614070000 \\
\hline H & 4.798077000 & -2.166608000 & -0.415011000 & H & 5.787702000 & -2.147315000 & -0.320605000 \\
\hline $\mathrm{H}$ & 2.658797000 & -2.695002000 & -1.521106000 & H & 3.742462000 & -2.812746000 & -1.542457000 \\
\hline $\mathrm{H}$ & 1.130196000 & -0.910626000 & -2.527057000 & $\mathrm{H}$ & 2.170352000 & -1.156764000 & -2.701998000 \\
\hline $\mathrm{H}$ & 1.221829000 & -2.853817000 & -0.530272000 & $\mathrm{H}$ & 2.259293000 & -3.005701000 & -0.627182000 \\
\hline$P$ & -2.536996000 & 0.110251000 & 0.476261000 & $P$ & -1.614060000 & 0.023180000 & 0.126841000 \\
\hline C & -2.700063000 & 1.697942000 & 1.410484000 & c & -1.699474000 & 1.658825000 & 0.939433000 \\
\hline $\mathrm{H}$ & -2.005961000 & 1.627133000 & 2.255010000 & C & -0.655715000 & 2.044109000 & 1.798056000 \\
\hline $\mathrm{H}$ & -3.711313000 & 1.732322000 & 1.831977000 & H & 0.191309000 & 1.381612000 & 1.956833000 \\
\hline C & -2.411896000 & 2.952695000 & 0.576537000 & $\mathrm{H}$ & 0.099661000 & 3.564027000 & 3.118872000 \\
\hline $\mathrm{H}$ & -3.121317000 & 3.068296000 & -0.248036000 & C & -0.706752000 & 3.273161000 & 2.452706000 \\
\hline $\mathrm{H}$ & -1.402213000 & 2.929778000 & 0.154580000 & C & -1.791232000 & 4.130979000 & 2.247783000 \\
\hline $\mathrm{H}$ & -2.492377000 & 3.843409000 & 1.205488000 & $\mathrm{H}$ & -1.826207000 & 5.091088000 & 2.753295000 \\
\hline C & -2.991267000 & -1.209826000 & 1.687853000 & $\mathrm{H}$ & -3.669492000 & 4.420332000 & 1.229941000 \\
\hline $\mathrm{H}$ & -2.279902000 & -1.119638000 & 2.516235000 & C & -2.827130000 & 3.755055000 & 1.391896000 \\
\hline $\mathrm{H}$ & -3.981033000 & -0.966665000 & 2.090850000 & C & -2.786675000 & 2.521796000 & 0.737941000 \\
\hline $\mathrm{H}$ & -3.699552000 & -2.758034000 & 0.306230000 & $\mathrm{H}$ & -3.596239000 & 2.237600000 & 0.073851000 \\
\hline C & -2.968330000 & -2.630666000 & 1.109702000 & C & -2.117205000 & -1.228642000 & 1.358697000 \\
\hline H & -1.980316000 & -2.886213000 & 0.713801000 & C & -2.831742000 & -0.874699000 & 2.513134000 \\
\hline $\mathrm{H}$ & -3.703528000 & -0.825740000 & -1.399469000 & H & -3.075206000 & 0.164035000 & 2.710882000 \\
\hline C & -3.823728000 & 0.115891000 & -0.853055000 & C & -3.228103000 & -1.863009000 & 3.416168000 \\
\hline $\mathrm{H}$ & -3.534930000 & 0.908067000 & -1.552203000 & H & -3.778470000 & -1.583996000 & 4.309305000 \\
\hline $\mathrm{H}$ & -3.211888000 & -3.353822000 & 1.892679000 & C & -2.917194000 & -3.201818000 & 3.173749000 \\
\hline $\mathrm{H}$ & -5.592379000 & -0.505395000 & 0.286798000 & $\mathrm{H}$ & -3.225529000 & -3.966734000 & 3.879736000 \\
\hline C & -5.273686000 & 0.298002000 & -0.383311000 & $\mathrm{H}$ & -1.960343000 & -4.599082000 & 1.836103000 \\
\hline $\mathrm{H}$ & -5.941651000 & 0.285314000 & -1.249198000 & C & -2.205334000 & -3.558576000 & 2.025167000 \\
\hline \multirow[t]{8}{*}{$\mathrm{H}$} & -5.423325000 & 1.251866000 & 0.130440000 & C & -1.801825000 & -2.577104000 & 1.122013000 \\
\hline & & & & $\mathrm{H}$ & -1.244967000 & -2.859012000 & 0.232219000 \\
\hline & & & & C & -2.856646000 & 0.005912000 & -1.211592000 \\
\hline & & & & C & -2.524154000 & 0.560089000 & -2.459041000 \\
\hline & & & & $\mathrm{H}$ & -1.530885000 & 0.967994000 & -2.626630000 \\
\hline & & & & $\mathrm{H}$ & -3.203202000 & 1.014728000 & -4.448085000 \\
\hline & & & & C & -3.465936000 & 0.586173000 & -3.485958000 \\
\hline & & & & C & -4.740694000 & 0.052447000 & -3.279276000 \\
\hline
\end{tabular}




\begin{tabular}{|c|c|c|c|c|c|c|c|}
\hline & & & & $\mathrm{H}$ & -5.470703000 & 0.066216000 & -4.082683000 \\
\hline & & & & $\mathrm{H}$ & -6.062900000 & -0.922709000 & -1.882777000 \\
\hline & & & & c & -5.074329000 & -0.504032000 & -2.043424000 \\
\hline & & & & C & -4.137872000 & -0.529299000 & -1.008576000 \\
\hline & & & & H & -4.403214000 & -0.967507000 & -0.052087000 \\
\hline & & Int6 $\left(t \mathrm{Bu}_{3} \mathbf{P A u ^ { + }}\right)$ & & & & Int6 $\left.(\operatorname{IPrAu})^{+}\right)$ & \\
\hline C & 2.119339000 & -1.073877000 & -1.496298000 & C & 0.800987000 & -2.189073000 & -2.059968000 \\
\hline c & 2.601245000 & -2.248294000 & -0.655227000 & c & 0.474097000 & -3.508503000 & -1.373780000 \\
\hline C & 2.726041000 & 0.100006000 & -1.058178000 & C & 2.080701000 & -1.770923000 & -1.714329000 \\
\hline C & 3.429055000 & -0.115686000 & 0.201782000 & c & 2.631522000 & -2.608031000 & -0.648116000 \\
\hline c & 3.346783000 & -1.607141000 & 0.560445000 & c & 1.646558000 & -3.752033000 & -0.365400000 \\
\hline C & 4.701426000 & -2.298230000 & 0.792093000 & C & 2.237377000 & -5.160387000 & -0.554944000 \\
\hline c & 4.021665000 & 0.912642000 & 0.863304000 & c & 3.834527000 & -2.331657000 & -0.085802000 \\
\hline C & 4.744493000 & 0.762270000 & 2.172233000 & C & 4.448183000 & -3.138370000 & 1.024048000 \\
\hline C & 4.013444000 & 2.313039000 & 0.278721000 & C & 4.673161000 & -1.168432000 & -0.582982000 \\
\hline C & 2.830728000 & 1.416045000 & -1.770129000 & C & 2.946130000 & -0.746107000 & -2.387778000 \\
\hline C & 2.905084000 & 2.562224000 & -0.752445000 & C & 3.892560000 & -0.102818000 & -1.365183000 \\
\hline $\mathrm{Au}$ & 0.162551000 & -0.303133000 & -0.592332000 & $\mathrm{Au}$ & 0.118973000 & -0.503775000 & -0.692546000 \\
\hline $\mathrm{H}$ & 2.015071000 & 1.552515000 & -2.486300000 & $\mathrm{H}$ & 2.346831000 & -0.000454000 & -2.918047000 \\
\hline H & 3.762083000 & 1.386718000 & -2.356153000 & H & 3.534763000 & -1.281110000 & -3.148523000 \\
\hline $\mathrm{H}$ & 1.940038000 & 2.642085000 & -0.235298000 & $\mathrm{H}$ & 3.304547000 & 0.505889000 & -0.668199000 \\
\hline $\mathrm{H}$ & 3.073249000 & 3.513956000 & -1.264898000 & $\mathrm{H}$ & 4.587354000 & 0.574883000 & -1.870230000 \\
\hline H & 3.937934000 & 3.043464000 & 1.092368000 & H & 5.183367000 & -0.703896000 & 0.269054000 \\
\hline H & 4.998549000 & 2.492834000 & -0.180381000 & H & 5.478545000 & -1.580932000 & -1.211267000 \\
\hline $\mathrm{H}$ & 4.477141000 & 1.581698000 & 2.848677000 & $\mathrm{H}$ & 4.797556000 & -2.473267000 & 1.822558000 \\
\hline $\mathrm{H}$ & 5.831329000 & 0.825535000 & 2.026698000 & H & 5.333166000 & -3.682496000 & 0.668231000 \\
\hline $\mathrm{H}$ & 4.528118000 & -0.181444000 & 2.673807000 & $\mathrm{H}$ & 3.759133000 & -3.861900000 & 1.460280000 \\
\hline $\mathrm{H}$ & 2.733052000 & -1.726499000 & 1.464073000 & H & 1.269554000 & -3.665375000 & 0.662072000 \\
\hline H & 5.216748000 & -1.932031000 & 1.681953000 & H & 3.033972000 & -5.382978000 & 0.157934000 \\
\hline $\mathrm{H}$ & 4.555490000 & -3.376109000 & 0.916701000 & $\mathrm{H}$ & 1.456428000 & -5.916880000 & -0.425559000 \\
\hline $\mathrm{H}$ & 5.364288000 & -2.146472000 & -0.066304000 & $\mathrm{H}$ & 2.650217000 & -5.273092000 & -1.563129000 \\
\hline $\mathrm{H}$ & 3.300367000 & -2.843798000 & -1.257871000 & $\mathrm{H}$ & 0.451172000 & -4.303090000 & -2.131403000 \\
\hline $\mathrm{H}$ & 1.791113000 & -1.205945000 & -2.526478000 & $\mathrm{H}$ & 0.323335000 & -1.892728000 & -2.992030000 \\
\hline H & 1.805029000 & -2.931374000 & -0.346455000 & H & -0.506900000 & -3.516464000 & -0.890174000 \\
\hline$P$ & -2.038430000 & 0.076802000 & 0.227646000 & C & -0.904923000 & 0.874778000 & 0.402050000 \\
\hline C & -2.753108000 & 1.645484000 & -0.650658000 & N & -0.459057000 & 2.061292000 & 0.889712000 \\
\hline C & -2.086993000 & 2.913731000 & -0.073722000 & C & -1.480590000 & 2.723051000 & 1.558883000 \\
\hline $\mathrm{H}$ & -0.995012000 & 2.861951000 & -0.119649000 & $\mathrm{H}$ & -1.326734000 & 3.685225000 & 2.020120000 \\
\hline C & -3.192739000 & 0.952055000 & 2.756048000 & C & -2.580237000 & 1.929881000 & 1.481589000 \\
\hline H & -3.062192000 & 1.004569000 & 3.842771000 & H & -3.580822000 & 2.059778000 & 1.861219000 \\
\hline $\mathrm{H}$ & -2.388647000 & 3.130950000 & 0.951658000 & C & 1.834350000 & 2.279924000 & 1.741897000 \\
\hline $\mathrm{H}$ & -3.381248000 & 1.969055000 & 2.408032000 & C & 0.884710000 & 2.585839000 & 0.746142000 \\
\hline $\mathrm{H}$ & -2.398155000 & 3.766658000 & -0.686591000 & C & 1.168629000 & 3.409363000 & -0.361876000 \\
\hline H & -4.084236000 & 0.352046000 & 2.565611000 & C & 0.132567000 & 3.765160000 & -1.424507000 \\
\hline C & -4.284993000 & 1.775601000 & -0.511400000 & C & 0.571082000 & 3.296820000 & -2.825578000 \\
\hline $\mathrm{H}$ & -4.616423000 & 1.793908000 & 0.528420000 & $\mathrm{H}$ & 0.754308000 & 2.217284000 & -2.842969000 \\
\hline
\end{tabular}




\begin{tabular}{|c|c|c|c|c|c|c|c|}
\hline $\mathrm{H}$ & -4.594180000 & 2.721970000 & -0.969173000 & $\mathrm{H}$ & -0.207900000 & 3.521383000 & -3.561183000 \\
\hline $\mathrm{H}$ & -4.823201000 & 0.980084000 & -1.029910000 & $\mathrm{H}$ & 1.486988000 & 3.799897000 & -3.151422000 \\
\hline $\mathrm{H}$ & -5.045204000 & -0.708809000 & 0.442957000 & $\mathrm{H}$ & -0.797635000 & 3.240092000 & -1.187450000 \\
\hline C & -4.406709000 & -1.581007000 & 0.594167000 & $\mathrm{H}$ & -0.959517000 & 5.505406000 & -2.150219000 \\
\hline C & -2.375089000 & 1.604680000 & -2.150977000 & C & -0.179746000 & 5.275094000 & -1.417358000 \\
\hline $\mathrm{H}$ & -2.757365000 & 2.517355000 & -2.621757000 & $\mathrm{H}$ & -0.528880000 & 5.608578000 & -0.435067000 \\
\hline $\mathrm{H}$ & -2.800237000 & 0.759760000 & -2.688687000 & $\mathrm{H}$ & 0.703531000 & 5.868461000 & -1.674383000 \\
\hline $\mathrm{H}$ & -4.110715000 & -0.751235000 & -2.011781000 & $\mathrm{H}$ & 2.723886000 & 4.566225000 & -1.295478000 \\
\hline $\mathrm{H}$ & -3.855891000 & -2.488176000 & -1.942963000 & C & 2.467443000 & 3.924955000 & -0.458105000 \\
\hline C & -3.399507000 & -1.520381000 & -1.708497000 & C & 3.430690000 & 3.638610000 & 0.505354000 \\
\hline $\mathrm{H}$ & -2.496191000 & -1.431616000 & -2.319569000 & $\mathrm{H}$ & 4.428779000 & 4.056551000 & 0.413603000 \\
\hline $\mathrm{H}$ & -2.830943000 & -3.638950000 & -0.197524000 & C & 3.115478000 & 2.826163000 & 1.591493000 \\
\hline C & -2.236321000 & -2.762632000 & 0.083785000 & $\mathrm{H}$ & 3.872529000 & 2.619317000 & 2.341466000 \\
\hline $\mathrm{H}$ & -1.324114000 & -2.776355000 & -0.520684000 & $\mathrm{H}$ & 0.946990000 & 3.094976000 & 4.246362000 \\
\hline $\mathrm{H}$ & -1.956204000 & -2.882208000 & 1.128365000 & C & 1.615238000 & 2.228316000 & 4.263616000 \\
\hline $\mathrm{H}$ & -1.290138000 & 1.589859000 & -2.292712000 & $\mathrm{H}$ & 2.632115000 & 2.597416000 & 4.431413000 \\
\hline C & -3.084298000 & -1.498531000 & -0.196588000 & $\mathrm{H}$ & 1.341539000 & 1.606183000 & 5.121684000 \\
\hline $\mathrm{H}$ & -4.248701000 & -1.711826000 & 1.666188000 & C & 2.419696000 & 0.164591000 & 3.016275000 \\
\hline $\mathrm{H}$ & -4.964651000 & -2.456679000 & 0.244042000 & $\mathrm{H}$ & 2.323032000 & -0.437240000 & 2.106771000 \\
\hline C & -1.915646000 & 0.341142000 & 2.141578000 & $\mathrm{H}$ & 3.474234000 & 0.434826000 & 3.134262000 \\
\hline C & -1.621568000 & -1.007452000 & 2.833872000 & $\mathrm{H}$ & 2.143123000 & -0.460933000 & 3.871182000 \\
\hline $\mathrm{H}$ & -1.412827000 & -0.806059000 & 3.890202000 & C & 1.519194000 & 1.413506000 & 2.958133000 \\
\hline $\mathrm{H}$ & -0.739420000 & -1.501569000 & 2.415887000 & $\mathrm{H}$ & 0.487707000 & 1.059875000 & 2.870170000 \\
\hline $\mathrm{H}$ & -2.463523000 & -1.699805000 & 2.801691000 & $\mathrm{~N}$ & -2.210053000 & 0.797494000 & 0.768818000 \\
\hline C & -0.708022000 & 1.258036000 & 2.452300000 & C & -3.104647000 & -0.304237000 & 0.468706000 \\
\hline $\mathrm{H}$ & -0.807840000 & 2.265470000 & 2.052499000 & C & -3.834242000 & -0.268562000 & -0.736567000 \\
\hline $\mathrm{H}$ & -0.621513000 & 1.346230000 & 3.541143000 & C & -3.223128000 & -1.345952000 & 1.410907000 \\
\hline \multirow[t]{19}{*}{$\mathrm{H}$} & 0.228192000 & 0.831442000 & 2.079654000 & C & -2.455149000 & -1.371211000 & 2.729618000 \\
\hline & & & & $\mathrm{H}$ & -1.822602000 & -0.479554000 & 2.777419000 \\
\hline & & & & $\mathrm{H}$ & -0.810155000 & -2.607833000 & 1.984282000 \\
\hline & & & & C & -1.521104000 & -2.593623000 & 2.817220000 \\
\hline & & & & $\mathrm{H}$ & -2.083302000 & -3.532623000 & 2.795609000 \\
\hline & & & & $\mathrm{H}$ & -0.951592000 & -2.570447000 & 3.751999000 \\
\hline & & & & $\mathrm{H}$ & -2.843277000 & -1.282521000 & 4.872051000 \\
\hline & & & & C & -3.412047000 & -1.316734000 & 3.937364000 \\
\hline & & & & $\mathrm{H}$ & -4.056325000 & -0.432742000 & 3.899758000 \\
\hline & & & & $\mathrm{H}$ & -4.060248000 & -2.197903000 & 3.975416000 \\
\hline & & & & $\mathrm{H}$ & -4.224754000 & -3.209966000 & 1.801193000 \\
\hline & & & & C & -4.103564000 & -2.389808000 & 1.100483000 \\
\hline & & & & $\mathrm{H}$ & -5.511367000 & -3.209225000 & -0.303316000 \\
\hline & & & & C & -4.831641000 & -2.390706000 & -0.086228000 \\
\hline & & & & $\mathrm{H}$ & -5.279750000 & -1.349358000 & -1.907545000 \\
\hline & & & & C & -4.698527000 & -1.340954000 & -0.990904000 \\
\hline & & & & $\mathrm{H}$ & -2.988866000 & 1.589517000 & -1.375378000 \\
\hline & & & & C & -3.734327000 & 0.876461000 & -1.740375000 \\
\hline & & & & $\mathrm{C}$ & -5.072472000 & 1.633440000 & -1.858347000 \\
\hline
\end{tabular}




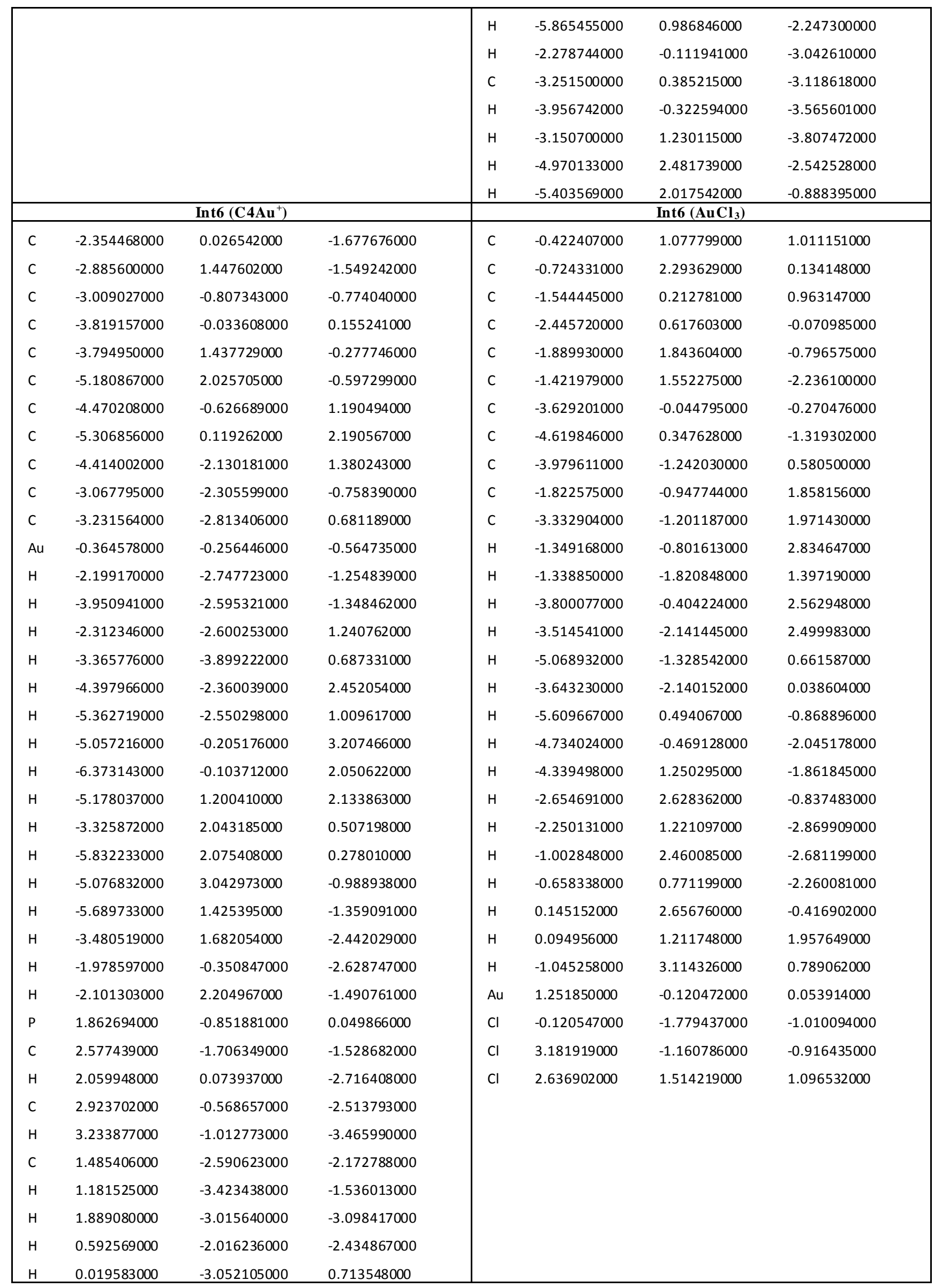




\begin{tabular}{|c|c|c|c|c|c|c|c|}
\hline c & 0.969201000 & -3.274430000 & 1.209715000 & & & & \\
\hline $\mathrm{H}$ & 1.550155000 & -3.938585000 & 0.565004000 & & & & \\
\hline $\mathrm{H}$ & 0.742885000 & -3.830536000 & 2.126212000 & & & & \\
\hline $\mathrm{H}$ & 3.626645000 & -3.419404000 & -0.617721000 & & & & \\
\hline c & 3.822395000 & -2.585172000 & -1.296216000 & & & & \\
\hline $\mathrm{H}$ & 4.119101000 & -3.018194000 & -2.258082000 & & & & \\
\hline $\mathrm{H}$ & 4.680895000 & -2.022136000 & -0.928222000 & & & & \\
\hline $\mathrm{H}$ & 3.756969000 & -2.922249000 & 1.509631000 & & & & \\
\hline C & 3.101143000 & -2.399027000 & 2.208487000 & & & & \\
\hline $\mathrm{H}$ & 2.902082000 & -3.084442000 & 3.040007000 & & & & \\
\hline $\mathrm{H}$ & 3.636327000 & -1.540860000 & 2.619154000 & & & & \\
\hline c & 1.748107000 & -1.998002000 & 1.586584000 & & & & \\
\hline C & 0.950639000 & -1.209560000 & 2.648606000 & & & & \\
\hline $\mathrm{H}$ & 0.843412000 & -1.831459000 & 3.544422000 & & & & \\
\hline $\mathrm{H}$ & 1.469408000 & -0.293704000 & 2.946695000 & & & & \\
\hline $\mathrm{H}$ & -0.051986000 & -0.943871000 & 2.299786000 & & & & \\
\hline C & 3.036124000 & 0.515606000 & 0.470943000 & & & & \\
\hline C & 4.381795000 & 0.153623000 & 0.696224000 & & & & \\
\hline H & 4.664064000 & -0.889259000 & 0.704329000 & & & & \\
\hline C & 5.380233000 & 1.098796000 & 0.907933000 & & & & \\
\hline $\mathrm{H}$ & 6.402143000 & 0.775391000 & 1.078732000 & & & & \\
\hline $\mathrm{H}$ & 5.816664000 & 3.208627000 & 1.048422000 & & & & \\
\hline C & 5.053309000 & 2.452821000 & 0.891948000 & & & & \\
\hline C & 3.731506000 & 2.831515000 & 0.685396000 & & & & \\
\hline $\mathrm{H}$ & 3.468153000 & 3.884270000 & 0.697301000 & & & & \\
\hline C & 2.700800000 & 1.893219000 & 0.483018000 & & & & \\
\hline C & 1.338217000 & 2.496773000 & 0.343333000 & & & & \\
\hline C & 1.062186000 & 3.343902000 & -0.743181000 & & & & \\
\hline $\mathrm{H}$ & 1.785808000 & 3.429472000 & -1.548741000 & & & & \\
\hline C & -0.108842000 & 4.102824000 & -0.777117000 & & & & \\
\hline $\mathrm{H}$ & -0.296766000 & 4.764788000 & -1.617282000 & & & & \\
\hline $\mathrm{H}$ & -1.912760000 & 4.651814000 & 0.271215000 & & & & \\
\hline C & -1.018335000 & 4.036086000 & 0.281218000 & & & & \\
\hline $\mathrm{H}$ & -1.453498000 & 3.144436000 & 2.197109000 & & & & \\
\hline C & -0.762347000 & 3.186754000 & 1.360267000 & & & & \\
\hline C & 0.404333000 & 2.419607000 & 1.389846000 & & & & \\
\hline $\mathrm{H}$ & 0.619701000 & 1.801570000 & 2.255356000 & & & & \\
\hline $\mathrm{H}$ & 3.742514000 & 0.057937000 & -2.153620000 & & & & \\
\hline \multicolumn{4}{|c|}{ Int7 } & \multicolumn{4}{|c|}{ TSf } \\
\hline C & 6.627929000 & 0.071248000 & -0.358286000 & C & -3.100869000 & -3.468888000 & -1.303817000 \\
\hline C & 5.598384000 & -0.115978000 & -1.151978000 & C & -1.586889000 & -3.559948000 & -1.068151000 \\
\hline C & 4.455920000 & -1.071399000 & -0.875253000 & C & -1.165737000 & -2.936441000 & 0.276686000 \\
\hline C & 3.118301000 & -0.331601000 & -0.690521000 & C & -1.180006000 & -1.437962000 & 0.206182000 \\
\hline C & -0.330043000 & 0.094985000 & -0.168881000 & C & -3.248314000 & -1.267594000 & -0.003139000 \\
\hline C & 0.683075000 & -0.614182000 & -0.302563000 & C & -3.773339000 & -2.269770000 & -0.694827000 \\
\hline C & 7.670861000 & 0.255161000 & 0.419311000 & C & -0.389405000 & -0.431792000 & 0.109221000 \\
\hline
\end{tabular}




\begin{tabular}{|c|c|c|c|c|c|c|c|}
\hline $\mathrm{H}$ & 5.538734000 & 0.448913000 & -2.085581000 & C & -3.544901000 & -0.168211000 & 0.750764000 \\
\hline $\mathrm{H}$ & 4.680757000 & -1.663200000 & 0.019301000 & C & -3.622469000 & -0.253750000 & 2.248045000 \\
\hline $\mathrm{H}$ & 4.361152000 & -1.776155000 & -1.714338000 & c & -3.587515000 & 1.176430000 & 0.133060000 \\
\hline $\mathrm{H}$ & 3.184630000 & 0.343177000 & 0.169530000 & $\mathrm{H}$ & -3.327145000 & -3.508415000 & -2.377041000 \\
\hline $\mathrm{H}$ & 2.906917000 & 0.287088000 & -1.570239000 & $\mathrm{H}$ & -3.596088000 & -4.351999000 & -0.874886000 \\
\hline C & 8.934092000 & -0.569248000 & 0.268376000 & $\mathrm{H}$ & -1.040190000 & -3.052679000 & -1.870203000 \\
\hline $\mathrm{H}$ & 9.790886000 & 0.083354000 & 0.058437000 & H & -1.279881000 & -4.611126000 & -1.089596000 \\
\hline $\mathrm{H}$ & 9.157588000 & -1.102669000 & 1.200989000 & $\mathrm{H}$ & -0.139409000 & -3.230268000 & 0.515534000 \\
\hline $\mathrm{H}$ & 8.850380000 & -1.300345000 & -0.538686000 & H & -1.802315000 & -3.301358000 & 1.089914000 \\
\hline C & 7.681594000 & 1.309396000 & 1.508477000 & $\mathrm{H}$ & -4.862541000 & -2.270490000 & -0.768408000 \\
\hline $\mathrm{H}$ & 7.863012000 & 0.847796000 & 2.487452000 & $\mathrm{H}$ & -3.558400000 & -1.280881000 & 2.609660000 \\
\hline $\mathrm{H}$ & 8.494697000 & 2.025954000 & 1.336553000 & $\mathrm{H}$ & -4.565249000 & 0.190360000 & 2.591502000 \\
\hline $\mathrm{H}$ & 6.739371000 & 1.860595000 & 1.552939000 & $\mathrm{H}$ & -2.813214000 & 0.334783000 & 2.703634000 \\
\hline $\mathrm{Au}$ & -1.635213000 & -1.629343000 & 0.080691000 & H & -3.942414000 & 1.154170000 & -0.899617000 \\
\hline$P$ & -3.189085000 & -3.342389000 & 0.367289000 & H & -2.536407000 & 1.561990000 & 0.097468000 \\
\hline $\mathrm{H}$ & -3.252550000 & -4.313236000 & -0.648197000 & $\mathrm{H}$ & -4.164030000 & 1.887752000 & 0.731213000 \\
\hline $\mathrm{H}$ & -3.041247000 & -4.144678000 & 1.512814000 & $\mathrm{Au}$ & -0.250272000 & 1.584696000 & -0.146033000 \\
\hline $\mathrm{H}$ & -4.538767000 & -2.959626000 & 0.469147000 & $P$ & 0.101264000 & 3.909681000 & -0.479363000 \\
\hline $\mathrm{Au}$ & -1.475636000 & 1.755160000 & -0.046338000 & H & -0.438975000 & 4.474173000 & -1.650638000 \\
\hline$P$ & -2.694923000 & 3.758551000 & 0.073654000 & $\mathrm{H}$ & -0.423239000 & 4.787530000 & 0.488614000 \\
\hline $\mathrm{H}$ & -2.994130000 & 4.249351000 & 1.357669000 & H & 1.424875000 & 4.385619000 & -0.558203000 \\
\hline $\mathrm{H}$ & -2.090852000 & 4.884583000 & -0.513814000 & $\mathrm{Au}$ & 1.680742000 & -0.830158000 & 0.136074000 \\
\hline $\mathrm{H}$ & -3.965266000 & 3.786157000 & -0.530720000 & $\mathrm{P}$ & 3.990809000 & -1.294856000 & 0.181706000 \\
\hline C & 1.945212000 & -1.327022000 & -0.475630000 & $\mathrm{H}$ & 4.889489000 & -0.219942000 & 0.036529000 \\
\hline $\mathrm{H}$ & 1.874057000 & -2.001847000 & -1.337689000 & $\mathrm{H}$ & 4.486260000 & -2.176634000 & -0.797590000 \\
\hline $\mathrm{H}$ & 2.145891000 & -1.945360000 & 0.408032000 & $\mathrm{H}$ & 4.497337000 & -1.895946000 & 1.349616000 \\
\hline \multicolumn{4}{|c|}{ Int8 } & \multicolumn{4}{|c|}{ TSg } \\
\hline C & -0.086435000 & 0.662478000 & 0.052739000 & C & -3.248915000 & 0.523950000 & 1.512167000 \\
\hline C & 2.557581000 & 0.390929000 & 0.674297000 & C & -0.590578000 & -0.124807000 & 0.134361000 \\
\hline C & 0.605350000 & 1.819664000 & 0.082710000 & C & -2.208142000 & 1.412934000 & 1.873985000 \\
\hline C & 2.061711000 & 1.710574000 & 0.144398000 & $\mathrm{H}$ & -2.147056000 & 1.705351000 & 2.922672000 \\
\hline C & 2.875425000 & 2.738356000 & -0.190287000 & $\mathrm{H}$ & -1.229934000 & 0.755240000 & 1.458369000 \\
\hline C & 2.339216000 & 4.094036000 & -0.570785000 & $\mathrm{H}$ & -2.042827000 & 2.250344000 & 1.197805000 \\
\hline C & 0.855980000 & 4.063800000 & -0.973442000 & C & -4.186305000 & -0.048297000 & 2.528208000 \\
\hline c & 0.015921000 & 3.209879000 & -0.002400000 & $\mathrm{H}$ & -3.801873000 & -1.009854000 & 2.902776000 \\
\hline C & 2.430968000 & 0.227317000 & 2.184578000 & H & -4.325469000 & 0.620486000 & 3.379901000 \\
\hline C & 3.874322000 & -0.115890000 & 0.123284000 & $\mathrm{H}$ & -5.162325000 & -0.273874000 & 2.082398000 \\
\hline $\mathrm{Au}$ & -2.054968000 & 0.184339000 & 0.061905000 & $\mathrm{Au}$ & 0.542718000 & 1.604298000 & -0.127164000 \\
\hline $\mathrm{Au}$ & 0.972787000 & -1.078910000 & -0.114132000 & $\mathrm{P}$ & 1.820028000 & 3.509586000 & -0.581731000 \\
\hline$P$ & 1.730073000 & -3.413781000 & -0.572351000 & H & 3.195780000 & 3.346971000 & -0.837960000 \\
\hline$P$ & -4.394490000 & -0.324215000 & 0.083621000 & $\mathrm{H}$ & 1.436375000 & 4.265632000 & -1.705298000 \\
\hline $\mathrm{H}$ & -5.014823000 & -0.648290000 & -1.138834000 & $\mathrm{H}$ & 1.861324000 & 4.515123000 & 0.402846000 \\
\hline H & -5.251136000 & 0.697015000 & 0.536917000 & $\mathrm{Au}$ & 1.061777000 & -1.403885000 & 0.163953000 \\
\hline H & -4.837965000 & -1.396375000 & 0.882875000 & $\mathrm{P}$ & 2.923414000 & -2.884820000 & 0.362195000 \\
\hline $\mathrm{H}$ & 2.507909000 & -4.077037000 & 0.398110000 & $\mathrm{H}$ & 2.679611000 & -4.151002000 & 0.929761000 \\
\hline $\mathrm{H}$ & 0.745009000 & -4.397827000 & -0.793697000 & $\mathrm{H}$ & 3.574168000 & -3.272389000 & -0.825878000 \\
\hline
\end{tabular}




\begin{tabular}{|c|c|c|c|c|c|c|c|}
\hline H & 2.534543000 & -3.623943000 & -1.709781000 & H & 4.036060000 & -2.483768000 & 1.129937000 \\
\hline $\mathrm{H}$ & 4.094242000 & -1.129457000 & 0.470577000 & C & -3.154738000 & -0.102437000 & 0.205331000 \\
\hline $\mathrm{H}$ & 3.912408000 & -0.082839000 & -0.967748000 & c & -4.280376000 & -0.391348000 & -0.510317000 \\
\hline $\mathrm{H}$ & 4.686106000 & 0.520200000 & 0.507174000 & $\mathrm{H}$ & -5.237556000 & -0.047209000 & -0.121932000 \\
\hline $\mathrm{H}$ & 2.572450000 & -0.809461000 & 2.502643000 & C & -4.323586000 & -1.108513000 & -1.817259000 \\
\hline $\mathrm{H}$ & 3.233660000 & 0.821213000 & 2.648019000 & $\mathrm{H}$ & -4.872258000 & -2.050058000 & -1.642364000 \\
\hline $\mathrm{H}$ & 1.475934000 & 0.591349000 & 2.564182000 & H & -4.947030000 & -0.549151000 & -2.527118000 \\
\hline $\mathrm{H}$ & 3.955958000 & 2.621716000 & -0.161529000 & C & -2.930051000 & -1.392265000 & -2.383167000 \\
\hline $\mathrm{H}$ & 2.945418000 & 4.505732000 & -1.387359000 & H & -2.552606000 & -0.504283000 & -2.904972000 \\
\hline $\mathrm{H}$ & 2.493363000 & 4.779202000 & 0.277753000 & $\mathrm{H}$ & -2.974938000 & -2.200622000 & -3.119751000 \\
\hline $\mathrm{H}$ & 0.764510000 & 3.644772000 & -1.983074000 & C & -1.958380000 & -1.761838000 & -1.251537000 \\
\hline $\mathrm{H}$ & 0.460124000 & 5.083700000 & -1.013596000 & $\mathrm{H}$ & -2.342018000 & -2.641347000 & -0.713082000 \\
\hline $\mathrm{H}$ & 0.029660000 & 3.668828000 & 0.997241000 & $\mathrm{H}$ & -0.984143000 & -2.027006000 & -1.662318000 \\
\hline $\mathrm{H}$ & -1.029020000 & 3.165669000 & -0.323513000 & C & -1.797276000 & -0.582822000 & -0.284949000 \\
\hline \multicolumn{4}{|c|}{ Int9 } & \multicolumn{4}{|c|}{ TSh } \\
\hline C & 1.718366000 & -0.398359000 & 0.396792000 & C & -0.313281000 & -0.593408000 & -0.018060000 \\
\hline C & 3.074335000 & -0.637925000 & -0.114962000 & C & -2.135951000 & -0.379726000 & 1.309294000 \\
\hline $\mathrm{Au}$ & -0.427673000 & 1.586963000 & -0.208774000 & C & -1.099153000 & -1.674815000 & -0.302160000 \\
\hline C & 0.632627000 & -0.259589000 & -0.464220000 & C & -2.368287000 & -1.507219000 & 0.352458000 \\
\hline c & 3.294723000 & -1.006925000 & -1.553362000 & c & -3.423211000 & -2.332778000 & 0.183785000 \\
\hline C & 2.877730000 & -2.400033000 & -1.972346000 & C & -3.302797000 & -3.532427000 & -0.721645000 \\
\hline H & 1.827575000 & -2.602286000 & -1.733698000 & C & -2.100601000 & -3.458673000 & -1.686283000 \\
\hline $\mathrm{H}$ & 3.021445000 & -2.549720000 & -3.045554000 & C & -0.802904000 & -2.971249000 & -1.001262000 \\
\hline $\mathrm{H}$ & 3.473022000 & -3.152297000 & -1.439363000 & C & -1.501986000 & -0.721002000 & 2.639945000 \\
\hline C & 3.897015000 & -0.151243000 & -2.391276000 & C & -3.215484000 & 0.679182000 & 1.405346000 \\
\hline $\mathrm{H}$ & 4.094725000 & -0.418140000 & -3.425983000 & $\mathrm{Au}$ & 1.745688000 & -0.603314000 & 0.082177000 \\
\hline $\mathrm{H}$ & 4.208758000 & 0.838386000 & -2.069344000 & $\mathrm{Au}$ & -0.609201000 & 1.442356000 & -0.219841000 \\
\hline$P$ & -1.423800000 & 3.719098000 & -0.121968000 & $P$ & -0.730796000 & 3.742712000 & -0.669356000 \\
\hline $\mathrm{H}$ & -0.843878000 & 4.664599000 & 0.744475000 & $\mathrm{P}$ & 4.127968000 & -0.710772000 & 0.232657000 \\
\hline $\mathrm{H}$ & -1.447138000 & 4.446381000 & -1.326622000 & H & 4.863148000 & -0.507750000 & -0.951556000 \\
\hline $\mathrm{H}$ & -2.775264000 & 3.808056000 & 0.263351000 & $\mathrm{H}$ & 4.685927000 & -1.928539000 & 0.667017000 \\
\hline $\mathrm{H}$ & 0.881506000 & -0.357668000 & -1.521337000 & $\mathrm{H}$ & 4.788006000 & 0.188030000 & 1.094212000 \\
\hline $\mathrm{Au}$ & -1.152164000 & -1.308379000 & 0.012957000 & $\mathrm{H}$ & -1.551700000 & 4.539175000 & 0.152346000 \\
\hline$P$ & -3.037376000 & -2.681726000 & 0.404562000 & $\mathrm{H}$ & 0.465686000 & 4.484184000 & -0.627569000 \\
\hline $\mathrm{H}$ & -3.481863000 & -3.464506000 & -0.677530000 & $\mathrm{H}$ & -1.214529000 & 4.104227000 & -1.940657000 \\
\hline $\mathrm{H}$ & -2.916446000 & -3.665380000 & 1.404225000 & H & -2.911800000 & 1.537064000 & 2.010233000 \\
\hline $\mathrm{H}$ & -4.244122000 & -2.068000000 & 0.792518000 & H & -3.569644000 & 1.019310000 & 0.430423000 \\
\hline C & 4.141016000 & -0.537217000 & 0.724052000 & $\mathrm{H}$ & -4.073113000 & 0.213876000 & 1.920955000 \\
\hline $\mathrm{H}$ & 5.127081000 & -0.755472000 & 0.317921000 & $\mathrm{H}$ & -0.998018000 & 0.148393000 & 3.072865000 \\
\hline C & 4.077369000 & -0.147168000 & 2.169683000 & $\mathrm{H}$ & -2.295888000 & -1.014814000 & 3.344137000 \\
\hline $\mathrm{H}$ & 4.279138000 & -1.046171000 & 2.775170000 & $\mathrm{H}$ & -0.785935000 & -1.540368000 & 2.566288000 \\
\hline $\mathrm{H}$ & 4.900537000 & 0.541417000 & 2.396982000 & $\mathrm{H}$ & -4.353073000 & -2.190848000 & 0.731519000 \\
\hline C & 2.723799000 & 0.462738000 & 2.548601000 & $\mathrm{H}$ & -4.231671000 & -3.654411000 & -1.292422000 \\
\hline H & 2.680314000 & 1.502781000 & 2.200687000 & $\mathrm{H}$ & -3.235027000 & -4.431574000 & -0.088476000 \\
\hline $\mathrm{H}$ & 2.599552000 & 0.483327000 & 3.635860000 & $\mathrm{H}$ & -2.344616000 & -2.767354000 & -2.502331000 \\
\hline C & 1.582699000 & -0.336670000 & 1.903679000 & $\mathrm{H}$ & -1.935195000 & -4.439812000 & -2.141960000 \\
\hline
\end{tabular}




\begin{tabular}{|c|c|c|c|c|c|c|c|}
\hline $\mathrm{H}$ & 1.617006000 & -1.369561000 & 2.282585000 & $\mathrm{H}$ & -0.469632000 & -3.714280000 & -0.261064000 \\
\hline $\mathrm{H}$ & 0.604793000 & 0.069888000 & 2.176431000 & $\mathrm{H}$ & 0.008921000 & -2.855223000 & -1.726553000 \\
\hline \multicolumn{4}{|c|}{ Int10 } & \multicolumn{4}{|c|}{ Int11 } \\
\hline c & -4.583176000 & -1.244402000 & -1.033840000 & C & 5.794936000 & -0.247709000 & -0.407410000 \\
\hline c & -3.770510000 & -0.414387000 & -2.056696000 & C & 4.751386000 & -0.012292000 & -1.169477000 \\
\hline c & -2.239659000 & -0.636918000 & -1.963142000 & C & 3.347675000 & -0.493296000 & -0.871888000 \\
\hline c & -1.841655000 & -0.534365000 & -0.531688000 & C & 2.390094000 & 0.709756000 & -0.611812000 \\
\hline c & -2.753350000 & -0.808507000 & 0.546087000 & c & -0.095376000 & -0.268764000 & -0.205805000 \\
\hline c & -4.040690000 & -1.165129000 & 0.376612000 & c & 1.020636000 & 0.250820000 & -0.387170000 \\
\hline C & -0.748681000 & -0.267782000 & 0.306377000 & C & 6.853207000 & -0.492540000 & 0.331214000 \\
\hline c & -1.650476000 & -0.556953000 & 1.592838000 & $\mathrm{H}$ & 4.880455000 & 0.561418000 & -2.090441000 \\
\hline C & -1.245654000 & -1.793478000 & 2.403665000 & H & 3.350521000 & -1.150707000 & 0.002000000 \\
\hline C & -1.893912000 & 0.644279000 & 2.512376000 & $\mathrm{H}$ & 2.959882000 & -1.069100000 & -1.721252000 \\
\hline $\mathrm{H}$ & -5.627500000 & -0.911635000 & -1.045278000 & $\mathrm{H}$ & 2.745698000 & 1.271412000 & 0.259483000 \\
\hline $\mathrm{H}$ & -4.612618000 & -2.303267000 & -1.337466000 & $\mathrm{H}$ & 2.417290000 & 1.388717000 & -1.472633000 \\
\hline $\mathrm{H}$ & -3.979054000 & 0.648560000 & -1.882692000 & c & 7.706143000 & -1.726476000 & 0.112709000 \\
\hline $\mathrm{H}$ & -4.115869000 & -0.642442000 & -3.069623000 & $\mathrm{H}$ & 8.736847000 & -1.436584000 & -0.126462000 \\
\hline $\mathrm{H}$ & -1.687262000 & 0.058298000 & -2.604600000 & $\mathrm{H}$ & 7.746028000 & -2.330838000 & 1.027554000 \\
\hline $\mathrm{H}$ & -1.990532000 & -1.650549000 & -2.312592000 & $\mathrm{H}$ & 7.324501000 & -2.348719000 & -0.699568000 \\
\hline $\mathrm{H}$ & -4.696971000 & -1.414704000 & 1.208368000 & c & 7.294407000 & 0.446180000 & 1.435951000 \\
\hline $\mathrm{H}$ & -2.016412000 & -2.039040000 & 3.142661000 & $\mathrm{H}$ & 7.317087000 & -0.079563000 & 2.398703000 \\
\hline $\mathrm{H}$ & -0.312771000 & -1.606808000 & 2.948110000 & $\mathrm{H}$ & 8.312571000 & 0.806033000 & 1.242720000 \\
\hline $\mathrm{H}$ & -1.101956000 & -2.668885000 & 1.761897000 & H & 6.633927000 & 1.311501000 & 1.526984000 \\
\hline H & -2.232348000 & 1.520965000 & 1.950545000 & $\mathrm{Au}$ & -0.972567000 & 1.730035000 & -0.007156000 \\
\hline $\mathrm{H}$ & -0.975029000 & 0.908942000 & 3.048394000 & $\mathrm{P}$ & -2.120217000 & 3.743918000 & 0.235941000 \\
\hline $\mathrm{H}$ & -2.657753000 & 0.408608000 & 3.261718000 & $\mathrm{H}$ & -2.091130000 & 4.623169000 & -0.861252000 \\
\hline $\mathrm{Au}$ & 1.099821000 & -1.246707000 & -0.066410000 & $\mathrm{H}$ & -3.501834000 & 3.655393000 & 0.484344000 \\
\hline $\mathrm{P}$ & 3.058661000 & -2.529124000 & -0.411632000 & H & -1.703453000 & 4.586173000 & 1.282159000 \\
\hline $\mathrm{H}$ & 3.028260000 & -3.478721000 & -1.450262000 & $\mathrm{Au}$ & -1.552826000 & -1.646798000 & 0.018432000 \\
\hline $\mathrm{H}$ & 4.255884000 & -1.852852000 & -0.716739000 & $\mathrm{P}$ & -3.161646000 & -3.340030000 & 0.264618000 \\
\hline $\mathrm{H}$ & 3.483971000 & -3.333710000 & 0.662338000 & $\mathrm{H}$ & -2.743340000 & -4.642326000 & -0.062669000 \\
\hline $\mathrm{Au}$ & 0.245600000 & 1.595503000 & -0.001730000 & H & -3.691258000 & -3.540701000 & 1.552538000 \\
\hline $\mathrm{P}$ & 1.163903000 & 3.756572000 & -0.262196000 & $\mathrm{H}$ & -4.338443000 & -3.233442000 & -0.499220000 \\
\hline $\mathrm{H}$ & 0.714453000 & 4.515178000 & -1.359402000 & & & & \\
\hline $\mathrm{H}$ & 0.945006000 & 4.665935000 & 0.789747000 & & & & \\
\hline $\mathrm{H}$ & 2.558373000 & 3.875363000 & -0.420207000 & & & & \\
\hline \multicolumn{4}{|c|}{ TSi } & \multicolumn{4}{|c|}{ Int12 } \\
\hline C & 0.820099000 & 1.805771000 & 0.043810000 & C & -0.803109000 & 1.801507000 & 0.063797000 \\
\hline c & 0.469798000 & 3.263428000 & 0.206199000 & C & -0.098573000 & 3.169158000 & 0.030469000 \\
\hline C & 1.358849000 & 4.128472000 & -0.689221000 & C & -1.105160000 & 4.124027000 & -0.657911000 \\
\hline C & 2.707587000 & 3.469900000 & -0.647810000 & C & -2.410982000 & 3.433395000 & -0.502753000 \\
\hline C & 2.779508000 & 2.224205000 & -0.198204000 & C & -2.266698000 & 2.127781000 & -0.070770000 \\
\hline $\mathrm{H}$ & 0.628644000 & 3.542670000 & 1.255209000 & $\mathrm{H}$ & 0.860099000 & 3.118066000 & -0.486552000 \\
\hline $\mathrm{H}$ & -0.592485000 & 3.389298000 & -0.014169000 & $\mathrm{H}$ & 0.095358000 & 3.518287000 & 1.053819000 \\
\hline $\mathrm{H}$ & 0.979539000 & 4.165995000 & -1.719439000 & $\mathrm{H}$ & -1.121927000 & 5.149988000 & -0.275218000 \\
\hline H & 1.405739000 & 5.161419000 & -0.328777000 & $\mathrm{H}$ & -0.895758000 & 4.204775000 & -1.739608000 \\
\hline $\mathrm{H}$ & 3.596681000 & 3.985889000 & -1.003554000 & $\mathrm{H}$ & -3.363234000 & 3.939501000 & -0.626987000 \\
\hline
\end{tabular}




\begin{tabular}{|c|c|c|c|c|c|c|c|}
\hline $\mathrm{Au}$ & -1.808438000 & 0.503138000 & 0.055046000 & $\mathrm{Au}$ & 1.868904000 & 0.422305000 & 0.057153000 \\
\hline$P$ & -4.161696000 & 0.382660000 & 0.102368000 & $P$ & 4.252697000 & 0.147350000 & 0.072233000 \\
\hline $\mathrm{H}$ & -4.793813000 & 0.696513000 & 1.320454000 & $\mathrm{H}$ & 4.981619000 & 0.725088000 & -0.986834000 \\
\hline $\mathrm{H}$ & -4.863395000 & 1.227540000 & -0.778185000 & $\mathrm{H}$ & 4.960031000 & 0.686282000 & 1.165777000 \\
\hline $\mathrm{H}$ & -4.768141000 & -0.854318000 & -0.190907000 & $\mathrm{H}$ & 4.802325000 & -1.151682000 & 0.054091000 \\
\hline C & 0.290337000 & 0.637256000 & 0.015741000 & C & -0.180319000 & 0.605724000 & 0.062487000 \\
\hline C & 3.594307000 & 1.200000000 & 0.192856000 & C & -3.411811000 & 1.431315000 & 0.392463000 \\
\hline C & 3.721466000 & -0.015136000 & -0.638648000 & C & -3.277976000 & 0.426452000 & 1.456333000 \\
\hline $\mathrm{H}$ & 4.619749000 & -0.590745000 & -0.400358000 & H & -4.233013000 & 0.121988000 & 1.887596000 \\
\hline $\mathrm{H}$ & 2.841524000 & -0.669492000 & -0.400216000 & $\mathrm{H}$ & -2.781871000 & -0.458512000 & 0.988397000 \\
\hline $\mathrm{H}$ & 3.661484000 & 0.192620000 & -1.708897000 & $\mathrm{H}$ & -2.563547000 & 0.733278000 & 2.226846000 \\
\hline C & 4.185997000 & 1.176989000 & 1.572632000 & C & -4.758204000 & 1.613220000 & -0.217246000 \\
\hline $\mathrm{H}$ & 5.254940000 & 0.937974000 & 1.515979000 & $\mathrm{H}$ & -5.522814000 & 1.761424000 & 0.553983000 \\
\hline H & 4.052366000 & 2.124227000 & 2.096692000 & H & -4.810326000 & 2.404523000 & -0.964858000 \\
\hline $\mathrm{H}$ & 3.717901000 & 0.377097000 & 2.166001000 & $\mathrm{H}$ & -5.022005000 & 0.669851000 & -0.722035000 \\
\hline $\mathrm{Au}$ & 0.669511000 & -1.362079000 & -0.058390000 & $\mathrm{Au}$ & -0.803179000 & -1.331938000 & -0.069086000 \\
\hline$P$ & 0.935763000 & -3.720930000 & -0.120784000 & $P$ & -1.281755000 & -3.679290000 & -0.300105000 \\
\hline $\mathrm{H}$ & 1.673553000 & -4.254948000 & -1.194485000 & $\mathrm{H}$ & -2.183786000 & -4.267564000 & 0.609567000 \\
\hline H & 1.600951000 & -4.320851000 & 0.965622000 & $\mathrm{H}$ & -1.860116000 & -4.107151000 & -1.512297000 \\
\hline $\mathrm{H}$ & -0.218658000 & -4.525829000 & -0.184673000 & $\mathrm{H}$ & -0.220446000 & -4.603540000 & -0.206089000 \\
\hline \multicolumn{4}{|c|}{$\mathbf{T S} \mathbf{j}$} & \multicolumn{4}{|c|}{ Int13 } \\
\hline C & 3.217943000 & -1.943466000 & 2.137569000 & C & -1.698342000 & -0.586102000 & 0.546932000 \\
\hline $\mathrm{H}$ & 3.338405000 & -1.454386000 & 3.114965000 & C & -1.544526000 & -0.567851000 & 2.057193000 \\
\hline $\mathrm{H}$ & 3.589230000 & -2.967529000 & 2.268615000 & C & -2.982544000 & -0.466612000 & 2.608142000 \\
\hline C & 1.767217000 & -1.893366000 & 1.616246000 & C & -3.821042000 & -0.781895000 & 1.409780000 \\
\hline $\mathrm{H}$ & 1.491555000 & -2.856007000 & 1.169956000 & C & -3.108472000 & -0.882543000 & 0.252000000 \\
\hline $\mathrm{H}$ & 1.033883000 & -1.675784000 & 2.395477000 & $\mathrm{H}$ & -0.875173000 & 0.224291000 & 2.400515000 \\
\hline C & 3.996224000 & -1.221270000 & 1.088385000 & $\mathrm{H}$ & -1.096688000 & -1.520555000 & 2.366226000 \\
\hline $\mathrm{H}$ & 5.080336000 & -1.149031000 & 1.108178000 & $\mathrm{H}$ & -3.181429000 & -1.147522000 & 3.443570000 \\
\hline C & 3.210825000 & -0.611891000 & 0.156526000 & H & -3.206546000 & 0.544221000 & 2.978627000 \\
\hline C & 3.580313000 & -0.017693000 & -1.094007000 & $\mathrm{H}$ & -4.897795000 & -0.903933000 & 1.474730000 \\
\hline C & 1.751466000 & -0.813449000 & 0.527260000 & $\mathrm{Au}$ & 1.192126000 & -1.241768000 & -0.075721000 \\
\hline C & 0.676053000 & -0.224909000 & -0.044833000 & C & -0.697857000 & -0.326690000 & -0.382966000 \\
\hline C & 2.695625000 & 0.972272000 & -1.599019000 & C & -3.689869000 & -1.167803000 & -1.084144000 \\
\hline $\mathrm{H}$ & 2.811160000 & 1.246522000 & -2.648338000 & C & -5.054744000 & -0.585063000 & -1.374627000 \\
\hline $\mathrm{H}$ & 1.588235000 & 0.450548000 & -1.314856000 & $\mathrm{H}$ & -5.058188000 & 0.503461000 & -1.244094000 \\
\hline $\mathrm{H}$ & 2.539018000 & 1.842380000 & -0.962010000 & $\mathrm{H}$ & -5.367221000 & -0.812567000 & -2.396376000 \\
\hline C & 4.578027000 & -0.717398000 & -1.968189000 & $\mathrm{H}$ & -5.816154000 & -0.992142000 & -0.698444000 \\
\hline $\mathrm{H}$ & 4.112656000 & -1.574560000 & -2.479043000 & c & -3.053438000 & -1.947753000 & -1.974052000 \\
\hline $\mathrm{H}$ & 4.982787000 & -0.051003000 & -2.733330000 & $\mathrm{H}$ & -3.499793000 & -2.172896000 & -2.937917000 \\
\hline $\mathrm{H}$ & 5.403104000 & -1.125757000 & -1.374243000 & $\mathrm{H}$ & -2.095890000 & -2.411162000 & -1.760413000 \\
\hline $\mathrm{Au}$ & -0.244197000 & 1.636329000 & 0.105712000 & $\mathrm{P}$ & 3.209414000 & -2.454717000 & 0.139539000 \\
\hline $\mathrm{P}$ & -1.271604000 & 3.718122000 & 0.380810000 & H & 3.257529000 & -3.446657000 & 1.137132000 \\
\hline H & -2.678586000 & 3.773796000 & 0.344473000 & $\mathrm{H}$ & 3.622050000 & -3.196156000 & -0.983529000 \\
\hline $\mathrm{H}$ & -1.016724000 & 4.390323000 & 1.590843000 & $\mathrm{H}$ & 4.390340000 & -1.743314000 & 0.427630000 \\
\hline $\mathrm{H}$ & -0.951273000 & 4.726950000 & -0.547441000 & $\mathrm{H}$ & -1.018106000 & -0.420463000 & -1.420072000 \\
\hline
\end{tabular}




\begin{tabular}{|c|c|c|c|c|c|c|c|}
\hline $\mathrm{Au}$ & -1.115164000 & -1.297681000 & -0.144380000 & $\mathrm{Au}$ & 0.215289000 & 1.595796000 & -0.134923000 \\
\hline$P$ & -3.114760000 & -2.566591000 & -0.406242000 & $P$ & 1.041620000 & 3.800423000 & -0.036061000 \\
\hline $\mathrm{H}$ & -3.050085000 & -3.715478000 & -1.219104000 & H & 1.832702000 & 4.230481000 & -1.118138000 \\
\hline $\mathrm{H}$ & -3.679978000 & -3.121101000 & 0.758726000 & H & 0.093178000 & 4.838769000 & 0.021704000 \\
\hline $\mathrm{H}$ & -4.250955000 & -1.939023000 & -0.956256000 & H & 1.870900000 & 4.146279000 & 1.048041000 \\
\hline \multicolumn{4}{|c|}{ TSk } & \multicolumn{4}{|c|}{ Int14 } \\
\hline C & -0.413266000 & 1.907491000 & 0.007114000 & C & -0.470204000 & 1.973917000 & -0.039600000 \\
\hline c & 0.192965000 & 3.267136000 & -0.310361000 & $\mathrm{Au}$ & 2.052234000 & 0.115820000 & 0.038063000 \\
\hline C & -0.964061000 & 4.005170000 & -1.046606000 & $\mathrm{P}$ & 4.337543000 & -0.594522000 & 0.087447000 \\
\hline c & -2.198256000 & 3.214379000 & -0.717776000 & H & 5.152922000 & -0.195173000 & -0.988963000 \\
\hline C & -1.871135000 & 2.087606000 & -0.011550000 & H & 5.122598000 & -0.160789000 & 1.173076000 \\
\hline $\mathrm{H}$ & 1.102862000 & 3.191561000 & -0.909730000 & H & 4.614016000 & -1.975882000 & 0.113347000 \\
\hline $\mathrm{H}$ & 0.446757000 & 3.806458000 & 0.611115000 & C & 0.142645000 & 0.774245000 & -0.004777000 \\
\hline H & -1.059304000 & 5.058201000 & -0.756068000 & C & -2.621251000 & 0.774986000 & 0.492331000 \\
\hline H & -0.831798000 & 4.010731000 & -2.140762000 & C & -2.671413000 & 0.651709000 & 2.009695000 \\
\hline $\mathrm{H}$ & -3.197786000 & 3.526128000 & -1.006074000 & $\mathrm{H}$ & -3.435918000 & 1.356580000 & 2.371805000 \\
\hline $\mathrm{Au}$ & 2.066376000 & 0.098624000 & 0.068803000 & H & -2.973884000 & -0.347676000 & 2.334370000 \\
\hline $\mathrm{P}$ & 4.367341000 & -0.572402000 & 0.117772000 & $\mathrm{H}$ & -1.722006000 & 0.912649000 & 2.478717000 \\
\hline $\mathrm{H}$ & 5.277162000 & 0.221413000 & -0.607804000 & C & -3.920052000 & 0.427979000 & -0.198593000 \\
\hline $\mathrm{H}$ & 5.016371000 & -0.602435000 & 1.368220000 & $\mathrm{H}$ & -4.652067000 & 1.217532000 & 0.034110000 \\
\hline $\mathrm{H}$ & 4.718478000 & -1.848147000 & -0.368585000 & $\mathrm{H}$ & -3.817519000 & 0.387294000 & -1.285273000 \\
\hline c & 0.119149000 & 0.667947000 & 0.036912000 & H & -4.345071000 & -0.510062000 & 0.170169000 \\
\hline C & -2.779806000 & 1.234032000 & 0.706436000 & $\mathrm{Au}$ & -1.100107000 & -0.851924000 & -0.079919000 \\
\hline c & -2.519287000 & 0.897921000 & 2.149290000 & $P$ & -2.112453000 & -3.128688000 & -0.385683000 \\
\hline $\mathrm{H}$ & -3.099854000 & 1.623806000 & 2.744741000 & H & -3.009311000 & -3.610992000 & 0.589182000 \\
\hline $\mathrm{H}$ & -2.897009000 & -0.090056000 & 2.423797000 & H & -2.878424000 & -3.355702000 & -1.546569000 \\
\hline $\mathrm{H}$ & -1.469254000 & 0.977592000 & 2.422133000 & $\mathrm{H}$ & -1.238964000 & -4.233008000 & -0.461422000 \\
\hline C & -4.199875000 & 1.089855000 & 0.253153000 & C & 0.022881000 & 3.395324000 & -0.240249000 \\
\hline $\mathrm{H}$ & -4.850441000 & 1.734652000 & 0.863643000 & $\mathrm{H}$ & 0.397879000 & 3.800647000 & 0.707063000 \\
\hline $\mathrm{H}$ & -4.341567000 & 1.333054000 & -0.800529000 & $\mathrm{H}$ & 0.837037000 & 3.460916000 & -0.966809000 \\
\hline $\mathrm{H}$ & -4.549784000 & 0.067758000 & 0.431838000 & C & -1.260236000 & 4.163899000 & -0.689184000 \\
\hline $\mathrm{Au}$ & -1.122458000 & -0.958925000 & -0.111968000 & H & -1.382197000 & 5.114007000 & -0.156798000 \\
\hline $\mathrm{P}$ & -2.310960000 & -3.053222000 & -0.483471000 & $\mathrm{H}$ & -1.237254000 & 4.415151000 & -1.759014000 \\
\hline $\mathrm{H}$ & -3.463849000 & -3.342344000 & 0.275712000 & C & -2.397803000 & 3.204698000 & -0.409756000 \\
\hline $\mathrm{H}$ & -2.818236000 & -3.297854000 & -1.775832000 & H & -3.439655000 & 3.477353000 & -0.544691000 \\
\hline $\mathrm{H}$ & -1.596586000 & -4.251584000 & -0.279930000 & C & -1.922335000 & 2.007838000 & -0.000217000 \\
\hline \multicolumn{4}{|c|}{ TSI } & \multicolumn{4}{|c|}{ Int15 } \\
\hline C & 0.143987000 & 0.760564000 & -0.127741000 & C & -0.000528000 & 0.922782000 & 0.186052000 \\
\hline C & 1.926466000 & 1.363789000 & 1.189139000 & C & -0.001165000 & 1.989512000 & 1.394614000 \\
\hline C & 0.536146000 & 2.005228000 & -0.549410000 & C & -0.001310000 & 1.990063000 & -0.733578000 \\
\hline C & 1.737940000 & 2.384478000 & 0.112587000 & C & -0.001976000 & 3.021785000 & 0.245365000 \\
\hline C & 1.217855000 & 1.616178000 & 2.498597000 & C & -1.264160000 & 1.998263000 & 2.261133000 \\
\hline C & 3.297566000 & 0.744239000 & 1.333778000 & C & 1.261922000 & 1.999900000 & 2.260961000 \\
\hline $\mathrm{Au}$ & -1.800275000 & 0.109101000 & 0.054073000 & $\mathrm{Au}$ & -1.495025000 & -0.570276000 & -0.030507000 \\
\hline $\mathrm{Au}$ & 1.105841000 & -1.064589000 & -0.195279000 & $\mathrm{Au}$ & 1.495834000 & -0.568361000 & -0.030583000 \\
\hline$P$ & 2.024035000 & -3.207454000 & -0.452730000 & $P$ & 3.294352000 & -2.092634000 & -0.223001000 \\
\hline$P$ & -4.084731000 & -0.549296000 & 0.283254000 & $\mathrm{P}$ & -3.291634000 & -2.096761000 & -0.222815000 \\
\hline
\end{tabular}




\begin{tabular}{|c|c|c|c|c|c|c|c|}
\hline $\mathrm{H}$ & -4.822455000 & -0.708465000 & -0.905750000 & $\mathrm{H}$ & -4.271122000 & -1.815223000 & -1.193893000 \\
\hline $\mathrm{H}$ & -4.939699000 & 0.315565000 & 0.993628000 & $\mathrm{H}$ & -4.099837000 & -2.268043000 & 0.916992000 \\
\hline $\mathrm{H}$ & -4.375695000 & -1.770308000 & 0.923853000 & $\mathrm{H}$ & -2.999550000 & -3.438791000 & -0.535316000 \\
\hline $\mathrm{H}$ & 3.102462000 & -3.574856000 & 0.375354000 & $\mathrm{H}$ & 4.102642000 & -2.263223000 & 0.916848000 \\
\hline $\mathrm{H}$ & 1.172418000 & -4.313097000 & -0.264637000 & $\mathrm{H}$ & 3.003783000 & -3.434927000 & -0.535780000 \\
\hline $\mathrm{H}$ & 2.561427000 & -3.508007000 & -1.718410000 & $\mathrm{H}$ & 4.273618000 & -1.809825000 & -1.193933000 \\
\hline $\mathrm{H}$ & 3.305673000 & -0.100736000 & 2.026581000 & $\mathrm{H}$ & 1.299237000 & 1.102715000 & 2.889175000 \\
\hline $\mathrm{H}$ & 3.735572000 & 0.445586000 & 0.379617000 & $\mathrm{H}$ & 2.170860000 & 2.033563000 & 1.651987000 \\
\hline $\mathrm{H}$ & 3.953154000 & 1.521552000 & 1.764749000 & $\mathrm{H}$ & 1.265633000 & 2.871510000 & 2.924794000 \\
\hline $\mathrm{H}$ & 1.053128000 & 0.680509000 & 3.041415000 & $\mathrm{H}$ & -1.300162000 & 1.101122000 & 2.889484000 \\
\hline $\mathrm{H}$ & 1.859650000 & 2.247277000 & 3.133912000 & $\mathrm{H}$ & -1.268976000 & 2.869965000 & 2.924837000 \\
\hline $\mathrm{H}$ & 0.260238000 & 2.121358000 & 2.367138000 & $\mathrm{H}$ & -2.173214000 & 2.030591000 & 1.652258000 \\
\hline $\mathrm{H}$ & 3.088785000 & 4.109345000 & 0.026557000 & $\mathrm{H}$ & -0.003907000 & 5.218543000 & 0.164135000 \\
\hline C & 2.205090000 & 3.578598000 & -0.314488000 & C & -0.003123000 & 4.247655000 & -0.322460000 \\
\hline C & 1.256056000 & 4.147014000 & -1.356447000 & C & -0.003349000 & 4.099984000 & -1.839718000 \\
\hline $\mathrm{H}$ & 1.756353000 & 4.218287000 & -2.331835000 & $\mathrm{H}$ & 0.872254000 & 4.594202000 & -2.279428000 \\
\hline $\mathrm{H}$ & 0.949547000 & 5.167535000 & -1.099075000 & $\mathrm{H}$ & -0.880345000 & 4.592260000 & -2.278850000 \\
\hline C & 0.042721000 & 3.154623000 & -1.393809000 & C & -0.001794000 & 2.552565000 & -2.120753000 \\
\hline $\mathrm{H}$ & -0.220497000 & 2.838384000 & -2.408172000 & $\mathrm{H}$ & 0.876783000 & 2.237804000 & -2.694653000 \\
\hline $\mathrm{H}$ & -0.852291000 & 3.616653000 & -0.960723000 & $\mathrm{H}$ & -0.879469000 & 2.235962000 & -2.695001000 \\
\hline
\end{tabular}

xyz at BP86/def2-TZVP level for (Me3PAu)+

$\begin{array}{llll}\text { Int1 } \text { Me }_{3} \text { PAu }^{+} \text {) } & & \\ 6 & -5.236061 & -0.895382 & 0.313926 \\ 6 & -4.437654 & 1.580391 & -0.109779 \\ 6 & -4.798370 & 0.329836 & 0.100857 \\ 6 & -0.745046 & -0.952435 & -0.548835 \\ 6 & -1.681482 & 0.128242 & -0.792585 \\ 6 & -1.963172 & 1.030739 & 0.431538 \\ 6 & -3.022496 & 2.090942 & 0.121585 \\ 6 & -5.431189 & 2.629628 & -0.567183 \\ 6 & -5.273426 & -1.942352 & -0.780212 \\ 6 & -5.759926 & -1.328781 & 1.667448 \\ 1 & -2.628756 & -0.356966 & -1.100520 \\ 1 & -1.344823 & 0.726558 & -1.656110 \\ 1 & -2.295547 & 0.398909 & 1.269408 \\ 1 & -1.026044 & 1.520544 & 0.740604 \\ 1 & -2.711273 & 2.685896 & -0.758079 \\ 1 & -3.051019 & 2.811705 & 0.958630 \\ 1 & -5.130517 & 3.057024 & -1.538424 \\ 1 & -6.439004 & 2.210288 & -0.671161 \\ 1 & -5.474198 & 3.464760 & 0.151881 \\ 1 & -4.678676 & -2.828276 & -0.500300 \\ 1 & -4.904124 & -1.553985 & -1.738589 \\ 1 & -6.306817 & -2.294895 & -0.932863 \\ 1 & -5.189593 & -2.192166 & 2.049034 \\ 1 & -6.809907 & -1.654438 & 1.583363 \\ 1 & -5.706312 & -0.519210 & 2.405685 \\ 1 & 0.212808 & -2.989441 & -0.205227 \\ 79 & 1.580728 & -0.505380 & -0.105187\end{array}$

$\begin{array}{lcrr}6 & -0.036357 & -1.946707 & -0.325248 \\ 15 & 3.591916 & 0.572437 & 0.202400 \\ 6 & 3.685045 & 1.515176 & 1.762068 \\ 1 & 4.674541 & 1.985386 & 1.860670 \\ 1 & 2.911903 & 2.294991 & 1.773995 \\ 1 & 3.517279 & 0.842397 & 2.613685 \\ 6 & 3.991388 & 1.757589 & -1.126976 \\ 1 & 3.225985 & 2.543935 & -1.168451 \\ 1 & 4.972493 & 2.218029 & -0.938702 \\ 1 & 4.014566 & 1.238031 & -2.094123 \\ 6 & 4.979398 & -0.613145 & 0.251027 \\ 1 & 5.928325 & -0.075815 & 0.394972 \\ 1 & 4.836165 & -1.320269 & 1.078926 \\ 1 & 5.022348 & -1.176985 & -0.690329\end{array}$

\begin{tabular}{lrrr}
\multicolumn{4}{l}{$\mathrm{TSa}\left(\mathrm{Me}_{3} \mathrm{PAu}^{+}\right)$} \\
6 & 3.642977 & -1.555938 & 0.109132 \\
6 & 4.623869 & 0.855869 & -0.237119 \\
6 & 3.942841 & -0.264490 & -0.095917 \\
6 & 1.569635 & 0.098869 & 0.268023 \\
6 & 1.883278 & 1.369959 & 0.923378 \\
6 & 2.546803 & 2.395833 & -0.025419 \\
6 & 4.066648 & 2.256108 & -0.112524 \\
6 & 6.118517 & 0.781984 & -0.492802 \\
6 & 3.746604 & -2.180892 & 1.478455 \\
6 & 3.253296 & -2.465682 & -1.020474 \\
1 & 2.483211 & 1.214144 & 1.834371 \\
1 & 0.904205 & 1.756346 & 1.261231
\end{tabular}




$\begin{array}{lrrr}1 & 2.092603 & 2.293857 & -1.022667 \\ 1 & 2.305641 & 3.408045 & 0.331077 \\ 1 & 4.530857 & 2.700303 & 0.788288 \\ 1 & 4.442399 & 2.861613 & -0.954363 \\ 1 & 6.661583 & 1.356331 & 0.275037 \\ 1 & 6.488761 & -0.250075 & -0.487033 \\ 1 & 6.358815 & 1.233984 & -1.468430 \\ 1 & 2.785795 & -2.627870 & 1.779793 \\ 1 & 4.062762 & -1.460325 & 2.241601 \\ 1 & 4.480779 & -3.003501 & 1.448104 \\ 1 & 2.368020 & -3.074128 & -0.767073 \\ 1 & 4.071838 & -3.187285 & -1.187625 \\ 1 & 3.080833 & -1.924127 & -1.957897 \\ 1 & 0.886738 & -1.626673 & -0.802997 \\ 79 & -1.313605 & -0.194041 & -0.080262 \\ 6 & 0.719779 & -0.698118 & -0.258477 \\ 15 & -3.578848 & 0.207111 & 0.023642 \\ 6 & -4.117163 & 1.644363 & -0.969787 \\ 1 & -5.206004 & 1.779283 & -0.891771 \\ 1 & -3.613576 & 2.552513 & -0.612488 \\ 1 & -3.846652 & 1.487763 & -2.022599 \\ 6 & -4.193643 & 0.530218 & 1.714829 \\ 1 & -3.698271 & 1.419478 & 2.126940 \\ 1 & -5.281232 & 0.694388 & 1.703444 \\ 1 & -3.963863 & -0.327415 & 2.361124 \\ 6 & -4.573421 & -1.198737 & -0.588631 \\ 1 & -5.646678 & -0.966247 & -0.525867 \\ 1 & -4.310210 & -1.411513 & -1.633426 \\ 1 & -4.360984 & -2.092408 & 0.013300\end{array}$

\section{Int2( $\left.\mathrm{Me}_{3} \mathrm{PAu}^{+}\right)$}

\begin{tabular}{|c|c|c|c|}
\hline 5 & 3.633329 & -1.514958 & 0.160176 \\
\hline & 4.164758 & 0.872091 & -0.238615 \\
\hline 6 & 3.231250 & -0.156474 & 0.020345 \\
\hline 6 & 1.815857 & 0.219065 & 0.307657 \\
\hline 6 & 1.626737 & 1.528035 & 1.033244 \\
\hline 6 & 2.298803 & 2.630638 & 0.205623 \\
\hline 6 & 3.773614 & 2.302196 & -0.029098 \\
\hline 6 & 5.601743 & 0.710580 & -0.623509 \\
\hline 6 & 2.885761 & -2.443244 & 1.052788 \\
\hline 6 & 4.808344 & -2.138769 & -0.512735 \\
\hline 1 & 2.076544 & 1.490247 & 2.042887 \\
\hline 1 & 0.553476 & 1.728057 & 1.155032 \\
\hline 1 & 1.781524 & 2.712526 & -0.763238 \\
\hline 1 & 2.219578 & 3.611389 & 0.697822 \\
\hline 1 & 4.390027 & 2.632186 & 0.836287 \\
\hline 1 & 4.199890 & 2.888216 & -0.865558 \\
\hline 1 & 6.133440 & 1.665819 & -0.526691 \\
\hline 1 & 6.135695 & -0.048308 & -0.036397 \\
\hline 1 & 5.679161 & 0.402479 & -1.681123 \\
\hline 1 & 2.322878 & -3.182958 & 0.455432 \\
\hline 1 & 2.169449 & -1.932738 & 1.704727 \\
\hline 1 & 3.611047 & -3.039218 & 1.634692 \\
\hline 1 & 4.616051 & -3.210711 & -0.669051 \\
\hline 1 & 5.696743 & -2.096467 & 0.147950 \\
\hline 1 & 5.077359 & -1.674869 & -1.466561 \\
\hline 1 & 1.118446 & -1.381280 & -0.821445 \\
\hline
\end{tabular}

79

6

15

1

1

6

6

1

1

\section{$\mathrm{TSb}\left(\mathrm{Me}_{3} \mathrm{PAu}^{+}\right)$}

6

6

6

6

6

6

6

1

1

1

1

1

1
1

1
1

1
1

1

1

1

1

1

1

6

79

15

1

6

1

1

1

6

1

1

1

6

1

1

$\begin{array}{rrr}1.215571 & -0.202676 & -0.105932 \\ 0.803731 & -0.512832 & -0.220468 \\ -3.526375 & 0.090335 & -0.025013 \\ -4.145075 & 1.474769 & -1.054763 \\ -5.238862 & 1.562619 & -0.981145 \\ -3.684242 & 2.415216 & -0.723543 \\ -3.865993 & 1.305543 & -2.103594 \\ -4.192865 & 0.437075 & 1.646780 \\ -3.738506 & 1.355758 & 2.041862 \\ -5.285760 & 0.557452 & 1.618358 \\ -3.937945 & -0.390746 & 2.322270 \\ -4.485945 & -1.361484 & -0.600025 \\ -5.566993 & -1.165062 & -0.548790 \\ -4.210409 & -1.597126 & -1.636901 \\ -4.247094 & -2.231788 & 0.026128\end{array}$

$\begin{array}{lll}-3.436772 & -1.657144 & 0.076877\end{array}$ $\begin{array}{llll}-4.299632 & 0.696250 & -0.007744\end{array}$ $\begin{array}{lll}-3.250313 & -0.213846 & -0.046068\end{array}$ $\begin{array}{lll}-1.857865 & 0.305705 & -0.133676\end{array}$ $\begin{array}{lll}-1.663195 & 1.658595 & 0.508165\end{array}$ $\begin{array}{llll}-2.647673 & 2.648530 & -0.131549\end{array}$ $\begin{array}{lll}-4.082606 & 2.176957 & 0.093380\end{array}$ $\begin{array}{llll}-5.728636 & 0.288062 & -0.156690\end{array}$ $\begin{array}{llll}-2.550999 & -2.500801 & -0.601197\end{array}$ $\begin{array}{lll}-4.268199 & -2.207637 & 1.205147\end{array}$ $\begin{array}{lll}-0.626358 & 1.985447 & 0.366113\end{array}$ $\begin{array}{lll}-1.857044 & 1.600960 & 1.595735\end{array}$ $\begin{array}{llll}-2.503017 & 3.652673 & 0.292328\end{array}$ $\begin{array}{llll}-2.443365 & 2.722991 & -1.212094\end{array}$ $\begin{array}{lll}-4.796148 & 2.703673 & -0.561026\end{array}$ $\begin{array}{lll}-4.404361 & 2.438618 & 1.124686\end{array}$ $\begin{array}{llll}-6.090091 & 0.629028 & -1.143584\end{array}$ $\begin{array}{lll}-5.895405 & -0.791226 & -0.097739\end{array}$ $\begin{array}{lll}-6.359647 & 0.806996 & 0.583186\end{array}$ $\begin{array}{llll}-1.415632 & -1.716663 & -0.362418\end{array}$ $\begin{array}{llll}-2.400265 & -2.366395 & -1.675686\end{array}$ $\begin{array}{llll}-2.496293 & -3.547406 & -0.284085\end{array}$ $\begin{array}{lll}-3.630120 & -2.846054 & 1.840324\end{array}$ $\begin{array}{llll}-5.071558 & -2.863721 & 0.832656\end{array}$ $\begin{array}{llll}-4.703275 & -1.430716 & 1.844243\end{array}$ $\begin{array}{lll}-0.794775 & -0.437274 & -0.643014\end{array}$ $\begin{array}{lll}1.212974 & -0.129532 & -0.210995\end{array}$ $\begin{array}{llll}3.481776 & 0.087902 & 0.241796\end{array}$ $\begin{array}{llll}-0.889917 & -0.859289 & -1.652075\end{array}$ $\begin{array}{llll}4.543524 & -0.082426 & -1.240436\end{array}$ $\begin{array}{llll}5.606010 & 0.020688 & -0.975384\end{array}$ $\begin{array}{llll}4.379557 & -1.066652 & -1.699633\end{array}$ $\begin{array}{llll}4.277214 & 0.689569 & -1.974926\end{array}$ $\begin{array}{llll}4.131431 & -1.156459 & 1.418442\end{array}$ $\begin{array}{llll}3.601933 & -1.070547 & 2.376875\end{array}$ $\begin{array}{lll}3.961912 & -2.165433 & 1.018902\end{array}$ $\begin{array}{lll}5.208727 & -1.009935 & 1.585215\end{array}$ $\begin{array}{lll}3.961680 & 1.698692 & 0.969182\end{array}$ $\begin{array}{llll}3.422615 & 1.852242 & 1.913748\end{array}$ $\begin{array}{llll}5.044072 & 1.731809 & 1.161449\end{array}$ 
$\begin{array}{lll}1.096949 & 2.581329 & -0.243541\end{array}$ Int3( $\left.\mathrm{Me}_{3} \mathrm{PAu}^{+}\right)$

\begin{tabular}{|c|c|c|c|c|}
\hline 6 & 2.406036 & 1.794113 & -0.313587 & 1 \\
\hline 6 & 3.624651 & -0.064242 & 0.842425 & 1 \\
\hline 6 & 2.620341 & 0.334674 & -0.012728 & 1 \\
\hline 6 & 1.773623 & -0.653157 & -0.659139 & 1 \\
\hline 6 & 1.914687 & -2.101280 & -0.245384 & 1 \\
\hline 6 & 3.373862 & -2.458082 & 0.047778 & 1 \\
\hline 6 & 3.923218 & -1.514461 & 1.114989 & 1 \\
\hline 6 & 4.523067 & 0.908459 & 1.537180 & 1 \\
\hline 6 & 3.049512 & 2.385962 & -1.329766 & 1 \\
\hline 6 & 1.445810 & 2.533373 & 0.584833 & 1 \\
\hline 1 & 1.482946 & -2.753741 & -1.017892 & 1 \\
\hline 1 & 1.319704 & -2.261278 & 0.672916 & 1 \\
\hline 1 & 3.449297 & -3.504955 & 0.374966 & 1 \\
\hline 1 & 3.971863 & -2.364316 & -0.873418 & 15 \\
\hline 1 & 5.011634 & -1.638436 & 1.235945 & 79 \\
\hline 1 & 3.494252 & -1.765994 & 2.106038 & 6 \\
\hline 1 & 5.541913 & 0.836404 & 1.117283 & 1 \\
\hline 1 & 4.195727 & 1.949679 & 1.449335 & 1 \\
\hline 1 & 4.620565 & 0.639488 & 2.602133 & 1 \\
\hline 1 & 0.932571 & 0.681145 & -2.122019 & 6 \\
\hline 1 & 3.748348 & 1.837853 & -1.964911 & 1 \\
\hline 1 & 2.899779 & 3.443953 & -1.556423 & 1 \\
\hline 1 & 1.331103 & 3.580353 & 0.274019 & 1 \\
\hline 1 & 1.788261 & 2.515759 & 1.632892 & 6 \\
\hline 1 & 0.448451 & 2.056411 & 0.576728 & 1 \\
\hline 6 & 0.854765 & -0.311768 & -1.668210 & 1 \\
\hline 79 & -0.941569 & -0.140936 & -0.424226 & 1 \\
\hline
\end{tabular}

$\begin{array}{llll}1.916563 & 2.285983 & 1.296854\end{array}$

$3.392545 \quad 3.697956-0.124564$

$3.263342 \quad 2.548345-1.461445$

$\begin{array}{llll}5.415523 & 2.170849 & -0.384139\end{array}$

$\begin{array}{lll}4.710276 & 2.129918 & 1.221139\end{array}$

$\begin{array}{llll}6.294257 & -0.232569 & -0.717396\end{array}$

$\begin{array}{llll}5.508461 & -1.517260 & 0.236949\end{array}$

$\begin{array}{llll}6.249029 & -0.114802 & 1.039730\end{array}$

$\begin{array}{llll}2.493092 & -3.428843 & -1.173248\end{array}$

$2.932101-1.958757-2.125638$

$\begin{array}{llll}4.122151 & -2.732730 & -1.071132\end{array}$

$\begin{array}{lll}1.748090 & -3.123807 & 1.228250\end{array}$

$\begin{array}{lll}3.419246 & -2.718367 & 1.638965\end{array}$

$\begin{array}{lll}2.119581 & -1.585218 & 2.088115\end{array}$

$\begin{array}{llll}1.233245 & -1.272332 & -0.498556\end{array}$

$\begin{array}{llll}-3.492637 & 0.130432 & 0.066215\end{array}$

$\begin{array}{llll}-1.182915 & -0.121848 & -0.078124\end{array}$

$-4.423681-1.441921-0.040424$

$\begin{array}{lll}-4.117404 & -2.110073 & 0.775605\end{array}$

$\begin{array}{lll}-5.505241 & -1.255433 & 0.031302\end{array}$

$\begin{array}{lll}-4.204699 & -1.936237 & -0.996348\end{array}$

$\begin{array}{lll}-4.048501 & 0.905832 & 1.627403\end{array}$

$\begin{array}{lll}-3.737606 & 0.286788 & 2.479671\end{array}$

$\begin{array}{lll}-3.585855 & 1.896571 & 1.731371\end{array}$

$\begin{array}{lll}-5.142814 & 1.015182 & 1.638860\end{array}$

$\begin{array}{llll}-4.192666 & 1.184119 & -1.255397\end{array}$

$\begin{array}{llll}-5.282243 & 1.278058 & -1.138726\end{array}$

$\begin{array}{lll}-3.737647 & 2.182795 & -1.211655\end{array}$

$\begin{array}{lll}-3.969597 & 0.744552 & -2.236824\end{array}$

$\begin{array}{lllll}15 & -2.998276 & 0.065239 & 0.587798\end{array}$

$1 \quad 0.527315 \quad-1.116626 \quad-2.336022$

$6 \quad-4.093647 \quad-1.359876 \quad 0.261151$

$1 \quad-5.070542-1.208550 \quad 0.743585$

$1 \quad-4.238751-1.475731-0.821223$

$\begin{array}{llll}-3.635300 & -2.278044 & 0.652226\end{array}$

$\begin{array}{llll}-3.921772 & 1.531867 & 0.010586\end{array}$

$\begin{array}{llll}-3.352282 & 2.442928 & 0.237535\end{array}$

$\begin{array}{llll}-4.068592 & 1.474037 & -1.076218\end{array}$

$\begin{array}{lll}-4.902252 & 1.583872 & 0.506374\end{array}$

$\begin{array}{lll}-2.932052 & 0.218219 & 2.408200\end{array}$

$\begin{array}{lll}-2.341840 & 1.101180 & 2.687060\end{array}$

$\begin{array}{lll}-3.948194 & 0.318324 & 2.817675\end{array}$

$1 \quad-2.455434-0.672682 \quad 2.838257$

\section{$\operatorname{lnt} 4\left(\mathrm{Me}_{3} \mathrm{PAu}^{+}\right)$}

$\begin{array}{lllll}6 & -2.760182 & 1.742242 & 0.021287\end{array}$

$\begin{array}{lllll}79 & 1.249237 & -0.124946 & -0.020801\end{array}$

$\begin{array}{lllll}6 & -4.375707 & -0.300110 & 0.012552\end{array}$

$\begin{array}{lllll}6 & -3.131854 & 0.248309 & -0.027688\end{array}$

$\begin{array}{lllll}6 & -1.966207 & -0.625991 & -0.135554\end{array}$

$\begin{array}{lllll}6 & -2.141723 & -2.149882 & -0.412037\end{array}$

$\begin{array}{lllll}6 & -3.349601 & -2.612154 & 0.404750\end{array}$

$\begin{array}{lllll}6 & -4.588773 & -1.800922 & 0.038095\end{array}$

$\begin{array}{lllll}6 & -5.653833 & 0.488196 & 0.052857\end{array}$

$\begin{array}{lllll}6 & -3.290372 & 2.482203 & 1.258812\end{array}$

$\begin{array}{lllll}6 & -3.078156 & 2.481182 & -1.290039\end{array}$

$\begin{array}{lllll}6 & -0.696824 & -0.347822 & -0.075245\end{array}$

$\begin{array}{lll}-0.696824 & -0.347822 & -0.075245 \\ -1.236494 & -2.721126 & -0.163800\end{array}$

$-2.318576-2.251884-1.494721$

$\begin{array}{llll}-3.513059 & -3.682879 & 0.202569\end{array}$

$\begin{array}{llll}-3.130973 & -2.516020 & 1.479788\end{array}$

$\begin{array}{lll}-5.405110 & -2.021383 & 0.746213\end{array}$

$\begin{array}{llll}-4.974229 & -2.113627 & -0.950942\end{array}$

$\begin{array}{llll}-5.533667 & 1.543848 & -0.206347\end{array}$

$\begin{array}{llll}-6.385702 & 0.043795 & -0.641247\end{array}$

$\begin{array}{lll}-6.107686 & 0.428790 & 1.057043\end{array}$

$\begin{array}{lll}-2.847979 & 3.488424 & 1.309722\end{array}$

$\begin{array}{lll}-3.032382 & 1.949880 & 2.185646\end{array}$

$\begin{array}{llll}-4.381143 & 2.609327 & 1.234988\end{array}$

$\begin{array}{llll}-2.673684 & 3.503818 & -1.256998\end{array}$ 


$\begin{array}{llll}1 & -4.161215 & 2.562308 & -1.461294 \\ 1 & -2.638650 & 1.967978 & -2.157547 \\ 1 & -1.651977 & 1.740189 & 0.114773 \\ 15 & 3.572500 & 0.156393 & 0.046689 \\ 6 & 4.331183 & -0.391241 & 1.615446 \\ 1 & 4.122007 & -1.457531 & 1.776078 \\ 1 & 5.419693 & -0.236793 & 1.591348 \\ 1 & 3.903184 & 0.176865 & 2.452239 \\ 6 & 4.067670 & 1.903779 & -0.158299 \\ 1 & 5.163364 & 1.994095 & -0.130986 \\ 1 & 3.697247 & 2.285021 & -1.119316 \\ 1 & 3.635203 & 2.510104 & 0.648895 \\ 6 & 4.448186 & -0.760115 & -1.269250 \\ 1 & 4.251459 & -1.835978 & -1.168692 \\ 1 & 4.089236 & -0.430360 & -2.253326 \\ 1 & 5.531431 & -0.583218 & -1.199975\end{array}$

\section{$\operatorname{TSd}\left(\mathrm{Me}_{3} \mathrm{PAu}^{+}\right)$}

$\begin{array}{lllll}6 & -3.201508 & -1.697210 & 0.311139\end{array}$

$\begin{array}{lllll}79 & 1.231886 & -0.139974 & 0.022422\end{array}$

$\begin{array}{lllll}6 & -4.387904 & 0.613145 & 0.042442\end{array}$

$\begin{array}{lllll}6 & -3.283945 & -0.181802 & 0.119091\end{array}$

$\begin{array}{lllll}6 & -1.948233 & 0.424123 & 0.171673\end{array}$

$\begin{array}{lllll}6 & -1.841327 & 1.935630 & 0.388419\end{array}$

$\begin{array}{lllll}6 & -2.876676 & 2.615818 & -0.509125\end{array}$

$\begin{array}{lllll}6 & -4.273240 & 2.108664 & -0.163712\end{array}$

$\begin{array}{lllll}6 & -5.817295 & 0.144721 & 0.170522\end{array}$

$\begin{array}{lllll}6 & -1.953782 & -2.223461 & -0.363178\end{array}$

$\begin{array}{lllll}6 & -4.382582 & -2.559344 & -0.177995\end{array}$

$\begin{array}{lllll}6 & -0.778849 & -0.196739 & 0.076825\end{array}$

$\begin{array}{llll}1 & -0.823296 & 2.293392 & 0.182905\end{array}$

$\begin{array}{lllll}1 & -2.056954 & 2.138792 & 1.451391\end{array}$

$\begin{array}{lllll}1 & -2.820052 & 3.707282 & -0.379659\end{array}$

$\begin{array}{lllll}1 & -2.643361 & 2.403728 & -1.564957\end{array}$

$\begin{array}{lllll}1 & -4.992959 & 2.397722 & -0.949238\end{array}$

$\begin{array}{lllll}1 & -4.645573 & 2.602539 & 0.754805\end{array}$

$1 \quad-6.163645-0.380801-0.732692$

$\begin{array}{llll}1 & -5.966638 & -0.527995 & 1.025195\end{array}$

$\begin{array}{lllll}1 & -6.480273 & 1.009554 & 0.309911\end{array}$

$1 \quad-1.617747 \quad-3.206516 \quad-0.001730$

$1 \quad-1.010884 \quad-1.580874-0.029538$

$1 \quad-1.967842 \quad-2.176276-1.459444$

$1 \quad-4.124918-3.625426 \quad-0.100615$

$\begin{array}{lllll}1 & -4.620285 & -2.346624 & -1.230091\end{array}$

$\begin{array}{lllll}1 & -5.283566 & -2.403246 & 0.423022\end{array}$

$1 \quad-3.058162-1.837561 \quad 1.396010$

$15 \quad 3.540406 \quad 0.104136-0.017911$

$\begin{array}{lllll}6 & 4.074666 & 1.687158 & -0.759725\end{array}$

$\begin{array}{lllll}1 & 3.664048 & 2.525958 & -0.181782\end{array}$

$\begin{array}{lllll}1 & 5.172202 & 1.756783 & -0.766660\end{array}$

$\begin{array}{lllll}1 & 3.701451 & 1.757701 & -1.790199\end{array}$

$64.392988-1.192853 \quad-0.984811$

$1 \quad 5.479396 \quad-1.021494-0.977966$

$14.180661 \quad-2.179626-0.551989$

$14.031297 \quad-1.180910-2.021682$

$\begin{array}{lllll}6 & 4.314234 & 0.059312 & 1.637334\end{array}$

$\begin{array}{lllll}1 & 3.900300 & 0.861709 & 2.262637\end{array}$

$\begin{array}{lllll}1 & 4.099005 & -0.903366 & 2.120086 \\ 1 & 5.403390 & 0.188468 & 1.556225\end{array}$

Int5( $\left.\mathrm{Me}_{3} \mathrm{PAu}^{+}\right)$

$\begin{array}{lllll}6 & 3.222447 & 1.918948 & -0.241691\end{array}$

$\begin{array}{lllll}79 & -1.199931 & 0.249419 & -0.130732\end{array}$

$\begin{array}{lllll}6 & 4.446173 & -0.329008 & -0.084230\end{array}$

$\begin{array}{lllll}6 & 3.299305 & 0.399587 & -0.100140\end{array}$

$\begin{array}{lllll}6 & 1.991484 & -0.241289 & 0.090583\end{array}$

$\begin{array}{lllll}6 & 1.971717 & -1.689842 & 0.597723\end{array}$

$\begin{array}{lllll}6 & 3.064347 & -2.476535 & -0.132302\end{array}$

$\begin{array}{lllll}6 & 4.432295 & -1.832190 & 0.086702\end{array}$

$\begin{array}{lllll}6 & 5.815945 & 0.268796 & -0.230773\end{array}$

$\begin{array}{lllll}6 & 3.310585 & 2.622443 & 1.126319\end{array}$

$\begin{array}{lllll}6 & 1.965358 & 2.290086 & -0.989827\end{array}$

$\begin{array}{lllll}6 & 0.804034 & 0.307152 & -0.113644\end{array}$

$\begin{array}{lllll}1 & 0.981810 & -2.142823 & 0.454913\end{array}$

$\begin{array}{lllll}1 & 2.172479 & -1.663157 & 1.682190\end{array}$

$1 \quad 3.066768-3.5152050 .232130$

$\begin{array}{lllll}1 & 2.831979 & -2.514143 & -1.208702\end{array}$

$\begin{array}{lllll}1 & 5.176049 & -2.272517 & -0.598328\end{array}$

$\begin{array}{lllll}1 & 4.806069 & -2.062833 & 1.102801\end{array}$

$1 \quad 6.274582-0.054918-1.180536$

$\begin{array}{lllll}1 & 5.830340 & 1.364342 & -0.198899\end{array}$

$\begin{array}{lllll}1 & 6.477534 & -0.103105 & 0.568877\end{array}$

$\begin{array}{lllll}1 & 3.273127 & 3.715368 & 1.007988\end{array}$

$\begin{array}{llll}1 & 4.247572 & 2.366590 & 1.638905\end{array}$

$\begin{array}{lllll}1 & 2.477658 & 2.318353 & 1.778206\end{array}$

$1 \quad 1.613434 \quad 3.320301 \quad-0.834398$

$\begin{array}{lllll}1 & 1.061446 & 1.687009 & -0.515076\end{array}$

$\begin{array}{lllll}1 & 1.955101 & 2.011983 & -2.050269\end{array}$

$1 \quad 4.069663 \quad 2.243735 \quad-0.869981$

$15 \quad-3.510736 \quad 0.008656 \quad-0.083850$

$\begin{array}{lllll}6 & -4.122357 & -1.333273 & -1.163436\end{array}$

$1 \quad-3.678578-2.289110-0.853993$

$1 \quad-5.217883-1.407076-1.101671$

$1 \quad-3.830677-1.134848-2.203490$

$\begin{array}{lllll}1 & -4.405499 & 1.510430 & -0.621882\end{array}$

$\begin{array}{lllll}1 & -5.491719 & 1.342481 & -0.580555\end{array}$

$\begin{array}{llll}1 & -4.145434 & 2.352580 & 0.033299\end{array}$

$1 \quad-4.118390 \quad 1.765682-1.650680$

$\begin{array}{llll}6 & -4.163626 & -0.373889 & 1.579548\end{array}$

$\begin{array}{lllll}1 & -3.723980 & -1.312230 & 1.943498\end{array}$

$\begin{array}{lllll}1 & -3.894108 & 0.429947 & 2.277632\end{array}$

$\begin{array}{llll}1 & -5.258187 & -0.475698 & 1.548042\end{array}$

\section{$\mathrm{TSe}\left(\mathrm{Me}_{3} \mathrm{PAu}^{+}\right)$}

\begin{tabular}{|c|c|c|c|}
\hline & -0.853420 & -0.440125 & 0.138769 \\
\hline & -1.793883 & -2.010035 & -0.741060 \\
\hline & -1.964722 & 0.345407 & 0.217480 \\
\hline & -3.274676 & -0.202914 & -0.100707 \\
\hline & -3.212879 & -1.715953 & -0.317684 \\
\hline & -3.616199 & -2.511074 & 0.940282 \\
\hline & -4.389089 & 0.576147 & -0.183901 \\
\hline & -5.754553 & 0.037514 & -0.484435 \\
\hline & -4.305078 & 2.069694 & 0.030727 \\
\hline & -1.865693 & 1.796479 & 0.618266 \\
\hline
\end{tabular}




\begin{tabular}{|c|c|c|c|c|c|c|c|}
\hline 6 & -2.895469 & 2.639501 & -0.145612 & 6 & 2.387892 & -1.582665 & 0.583552 \\
\hline 79 & 1.175513 & -0.173194 & 0.043574 & 6 & 3.756273 & -2.271730 & 0.714682 \\
\hline 15 & 3.460521 & 0.194057 & -0.067485 & 6 & 3.098461 & 0.941178 & 0.791798 \\
\hline 1 & -0.841642 & 2.166141 & 0.469076 & 6 & 3.927364 & 0.802894 & 2.032379 \\
\hline 1 & -2.070554 & 1.856471 & 1.703263 & 6 & 3.049407 & 2.328910 & 0.188501 \\
\hline 1 & -2.635301 & 2.651278 & -1.216762 & 6 & 1.705084 & 1.403517 & -1.746837 \\
\hline 1 & -2.857554 & 3.681224 & 0.204677 & 6 & 1.876889 & 2.573375 & -0.769150 \\
\hline 1 & -5.010571 & 2.570719 & -0.651995 & 79 & -0.802835 & -0.250641 & -0.351662 \\
\hline 1 & -4.681160 & 2.290446 & 1.048930 & 15 & -2.860884 & 0.227511 & 0.570121 \\
\hline 1 & -6.172252 & 0.528045 & -1.379366 & 1 & 0.835963 & 1.537764 & -2.407110 \\
\hline 1 & -6.446979 & 0.275851 & 0.341161 & 1 & 2.591730 & 1.341167 & -2.407317 \\
\hline 1 & -5.778090 & -1.047749 & -0.639155 & 1 & 0.948678 & 2.690816 & -0.184448 \\
\hline 1 & -3.874184 & -1.999239 & -1.154333 & 1 & 2.026711 & 3.512558 & -1.320640 \\
\hline 1 & -4.644310 & -2.268855 & 1.239043 & 1 & 3.037413 & 3.074775 & 0.999590 \\
\hline 1 & -3.561561 & -3.594479 & 0.758533 & 1 & 4.009469 & 2.497701 & -0.338656 \\
\hline 1 & -2.960298 & -2.267955 & 1.790507 & 1 & 3.730628 & 1.642843 & 2.717006 \\
\hline 1 & -1.407997 & -3.017880 & -0.521097 & 1 & 5.003966 & 0.851929 & 1.791662 \\
\hline 1 & -1.058422 & -1.598034 & 0.344116 & 1 & 3.745443 & -0.135252 & 2.568991 \\
\hline 1 & -1.484580 & -1.681331 & -1.735906 & 1 & 1.845676 & -1.688474 & 1.540146 \\
\hline 6 & 3.931967 & 1.932589 & 0.250155 & 1 & 4.348166 & -1.888815 & 1.555415 \\
\hline 1 & 5.023071 & 2.053480 & 0.184702 & 1 & 3.619371 & -3.351890 & 0.872808 \\
\hline 1 & 3.451236 & 2.588451 & -0.488047 & 1 & 4.347296 & -2.137802 & -0.204056 \\
\hline 1 & 3.595454 & 2.230147 & 1.252364 & 1 & 2.207273 & -2.837004 & -1.217645 \\
\hline 6 & 4.190798 & -0.203350 & -1.697445 & 1 & 0.599440 & -1.227585 & -2.386254 \\
\hline 1 & 4.015090 & -1.261379 & -1.934177 & 1 & 0.784366 & -2.934551 & -0.185376 \\
\hline 1 & 3.715590 & 0.409940 & -2.474795 & 6 & -3.909049 & -1.259769 & 0.728609 \\
\hline 1 & 5.273364 & -0.009084 & -1.694695 & 1 & -4.887263 & -0.993603 & 1.155216 \\
\hline 6 & 4.430547 & -0.784145 & 1.136290 & 1 & -4.058526 & -1.714934 & -0.259629 \\
\hline 1 & 5.503272 & -0.561660 & 1.039350 & 1 & -3.417945 & -1.994343 & 1.380641 \\
\hline 1 & 4.102342 & -0.545516 & 2.156832 & 6 & -3.840046 & 1.408880 & -0.421609 \\
\hline \multirow[t]{2}{*}{1} & 4.266832 & -1.855931 & 0.960997 & 1 & -3.305716 & 2.365130 & -0.499458 \\
\hline & & & & 1 & -3.989814 & 1.008617 & -1.433281 \\
\hline \multicolumn{4}{|c|}{ Int6 $\left(\mathrm{Me}_{3} \mathrm{PAu}^{+}\right)$} & 1 & -4.819804 & 1.579375 & 0.048554 \\
\hline 6 & 0.992374 & -1.080420 & -1.374529 & 6 & -2.774013 & 0.949013 & 2.246945 \\
\hline 6 & 1.549007 & -2.240426 & -0.560825 & 1 & -3.786202 & 1.143965 & 2.630973 \\
\hline 6 & 1.639515 & 0.107036 & -0.999469 & 1 & -2.257873 & 0.255274 & 2.923863 \\
\hline 6 & 2.439305 & -0.096714 & 0.197918 & 1 & -2.211970 & 1.891865 & 2.216699 \\
\hline
\end{tabular}

xyz for Au-triflate shuttle of scheme 9A at B3LYP/LAN2DZ(Au), 6-31G(d,p) $(\mathrm{C}, \mathrm{H}, \mathrm{O}, \mathrm{S}, \mathrm{P}, \mathrm{F})$ level

\begin{tabular}{cccc}
\multicolumn{4}{l}{ Int2+TfO- } \\
C & -2.844276 & 1.712972 & 0.403709 \\
C & -4.794610 & 0.428836 & -0.402751 \\
C & -3.467647 & 0.482821 & -0.046077 \\
C & -2.633703 & -0.733288 & 0.064747 \\
C & -3.320637 & -2.058888 & 0.363052 \\
C & -4.841487 & -1.918102 & 0.502293 \\
C & -5.397071 & -0.948633 & -0.560706 \\
C & -5.754948 & 1.578906 & -0.496305 \\
C & -2.185421 & 1.786758 & 1.723501 \\
C & -2.745481 & 2.898643 & -0.460224 \\
H & -2.893865 & -2.503786 & 1.269642 \\
H & -3.092495 & -2.754074 & -0.454319 \\
H & -5.329655 & -2.893568 & 0.408081 \\
H & -5.100197 & -1.528445 & 1.495268 \\
H & -6.489319 & -0.889459 & -0.508702 \\
H & -5.146708 & -1.329651 & -1.562930
\end{tabular}

$\begin{array}{lrrr}H & -6.551672 & 1.453429 & 0.249090 \\ H & -5.307709 & 2.560364 & -0.345998 \\ H & -6.252665 & 1.566221 & -1.474497 \\ H & -1.092920 & 1.960042 & 1.601700 \\ H & -2.331684 & 0.888154 & 2.321536 \\ H & -2.552379 & 2.678561 & 2.250778 \\ H & -1.678965 & 2.851800 & -0.798600 \\ H & -2.832387 & 3.844574 & 0.083691 \\ H & -3.377272 & 2.867904 & -1.347348 \\ \mathrm{C} & -1.312168 & -0.591749 & -0.194636 \\ \mathrm{Au} & 0.373594 & -1.718899 & -0.094095 \\ \mathrm{P} & 2.647211 & -2.429720 & 0.014284 \\ \mathrm{H} & -1.013507 & 0.412206 & -0.510217 \\ \mathrm{C} & 3.483424 & -1.646688 & 1.452295 \\ \mathrm{H} & 4.557738 & -1.857945 & 1.450520 \\ \mathrm{H} & 3.313661 & -0.568963 & 1.376399 \\ \mathrm{H} & 3.041488 & -2.010639 & 2.383309\end{array}$




$\begin{array}{cccc}\mathrm{C} & 3.578894 & -1.776182 & -1.429748 \\ \mathrm{H} & 3.204858 & -2.231118 & -2.350598 \\ \mathrm{H} & 3.398632 & -0.698257 & -1.467373 \\ \mathrm{H} & 4.651528 & -1.973704 & -1.334424 \\ \mathrm{C} & 3.108423 & -4.213281 & 0.111660 \\ \mathrm{H} & 2.724658 & -4.738748 & -0.766849 \\ \mathrm{H} & 4.194801 & -4.339681 & 0.159577 \\ \mathrm{H} & 2.656130 & -4.658848 & 1.001583 \\ \mathrm{O} & 0.848696 & 2.271207 & 1.445419 \\ \mathrm{O} & 2.318187 & 1.120840 & -0.229240 \\ \mathrm{~S} & 1.353806 & 2.210429 & 0.048575 \\ \mathrm{O} & 0.293503 & 2.397949 & -0.982256 \\ \mathrm{C} & 2.379566 & 3.752251 & -0.111181 \\ \mathrm{~F} & 1.621002 & 4.842480 & 0.091039 \\ \mathrm{~F} & 2.920416 & 3.837035 & -1.335232 \\ \mathrm{~F} & 3.371544 & 3.755974 & 0.791252\end{array}$

\section{$\mathrm{TSb}+\mathrm{TfO}^{-}$}

$\begin{array}{llll}\text { C } & -3.043467 & 1.091033 & 0.613888\end{array}$

$\begin{array}{llll}\text { C } & -4.677545 & -0.433849 & -0.415307\end{array}$

$\begin{array}{llll}\text { C } & -3.407443 & -0.213593 & 0.040209\end{array}$

$\begin{array}{llll}\text { C } & -2.343316 & -1.245002 & 0.047064\end{array}$

$\begin{array}{llll}\text { C } & -2.742664 & -2.636813 & -0.404584\end{array}$

$\begin{array}{llll}\text { C } & -4.226565 & -2.922775 & -0.163718\end{array}$

$\begin{array}{llll}\text { C } & -5.088828 & -1.835000 & -0.817380\end{array}$

$\begin{array}{llll}\text { C } & -5.788209 & 0.582292 & -0.457801\end{array}$

$\begin{array}{llll}\text { C } & -2.700599 & 1.199218 & 2.061157\end{array}$

$\begin{array}{llll}\text { C } & -2.931328 & 2.246227 & -0.205560\end{array}$

$\begin{array}{llll}\mathrm{H} & -2.108169 & -3.370267 & 0.102733\end{array}$

$\begin{array}{lllll}H & -2.533063 & -2.736152 & -1.479679\end{array}$

$\begin{array}{llll}\text { H } & -4.501568 & -3.906442 & -0.559082\end{array}$

H $\quad-4.431889-2.941850 \quad 0.914473$

H $\quad-6.148713-1.982469-0.574506$

$\begin{array}{llll}H & -5.019865 & -1.916599 & -1.914067\end{array}$

$\begin{array}{llll}\mathrm{H} & -6.594199 & 0.288181 & 0.227882\end{array}$

$\begin{array}{llll}H & -5.483107 & 1.595789 & -0.196081\end{array}$

H $\quad-6.236535 \quad 0.608870 \quad-1.459535$

$\begin{array}{llll}\mathrm{H} & -1.742973 & 1.724126 & 2.178224\end{array}$

$\begin{array}{llll}\text { H } & -2.662008 & 0.228716 & 2.553123\end{array}$

$\begin{array}{llll}\text { H } & -3.468223 & 1.827396 & 2.537149\end{array}$

H $\quad-1.769538 \quad 2.177972 \quad-0.512334$

$\begin{array}{llll}\mathrm{H} & -2.986022 & 3.212310 & 0.300674\end{array}$

H $\quad-3.431863 \quad 2.203699 \quad-1.172943$

$\begin{array}{llll}\text { C } & -1.070956 & -0.876707 & 0.312424\end{array}$

$\begin{array}{llll}\mathrm{Au} & 0.791017 & -1.672001 & 0.159975\end{array}$

$\begin{array}{lllll}\text { P } & 3.146195 & -1.895971 & -0.123356\end{array}$

$\begin{array}{llll}\text { H } & -0.930084 & 0.179680 & 0.544282\end{array}$

$\begin{array}{llll}\text { C } & 4.026135 & -0.875919 & 1.129879\end{array}$

$\begin{array}{llll}\text { H } & 5.103977 & -0.852179 & 0.939259\end{array}$

$\begin{array}{llll}H & 3.617191 & 0.136958 & 1.074549\end{array}$

$\begin{array}{llll}\text { H } & 3.841698 & -1.275202 & 2.130448\end{array}$

C $\quad 3.650672-1.126731-1.716899$

$\begin{array}{llll}\text { H } & 3.232434 & -1.693714 & -2.552487\end{array}$

H $3.236058-0.114873-1.736470$

H $4.740332 \quad-1.083600 \quad-1.813784$

$\begin{array}{llll}\text { C } & 4.006860 & -3.527308 & -0.090574\end{array}$

H $\quad 3.601871 \quad-4.170023 \quad-0.876673$ $\begin{array}{llll}\text { H } & 5.084777 & -3.410121 & -0.241314\end{array}$

H $\quad 3.830024 \quad-4.012700 \quad 0.872830$

$\begin{array}{llll}\text { O } & 0.226170 & 2.409825 & 1.356534\end{array}$

O $\quad 1.994231 \quad 1.710086 \quad-0.298402$

S $\quad 0.762682 \quad 2.471254-0.019278$

O $\quad-0.314481 \quad 2.304345 \quad-1.068006$

$\begin{array}{lllll}\text { C } & 1.255760 & 4.249761 & -0.239225\end{array}$

F $\quad 0.188867 \quad 5.046162 \quad-0.063016$

F $\quad \begin{array}{llll}1.744598 & 4.458831 & -1.467545\end{array}$

F $\quad 2.190411 \quad 4.586901 \quad 0.658525$

\section{Int2-H, TfOH}

$\begin{array}{llll}\text { C } & 3.925381 & -0.471942 & -1.583695\end{array}$

$\begin{array}{llll}\text { C } & 4.577246 & -0.113287 & 0.789390\end{array}$

C $\quad 3.596631-0.358225 \quad-0.119680$

$\begin{array}{llll}\text { C } & 2.183605 & -0.572781 & 0.286997\end{array}$

C $\quad \begin{array}{llll}1.879592 & -0.363788 & 1.761802\end{array}$

$\begin{array}{llll}\text { C } & 3.025839 & -0.832029 & 2.659237\end{array}$

$\begin{array}{llll}\text { C } & 4.310856 & -0.095910 & 2.279806\end{array}$

$\begin{array}{llll}\text { C } & 6.013706 & 0.145811 & 0.414655\end{array}$

$\begin{array}{llll}\text { C } & 4.284459 & -1.855582 & -2.076617\end{array}$

$\begin{array}{lllll}\text { C } & 3.902197 & 0.590690 & -2.395678\end{array}$

H $\quad 0.942969 \quad-0.868376 \quad 2.014740$

$\begin{array}{llll}H & 1.712923 & 0.710024 & 1.937927\end{array}$

H $\quad 2.782621 \quad-0.663298 \quad 3.714413$

H $\quad 3.173212 \quad-1.912220 \quad 2.531036$

H $\quad 5.173873 \quad-0.530421 \quad 2.802263$

$\begin{array}{llll}H & 4.259070 & 0.950204 & 2.622570\end{array}$

$\begin{array}{llll}H & 6.665309 & -0.650989 & 0.799430\end{array}$

H $\quad 6.1622190 .229068 \quad-0.662406$

H $\quad 6.365091 \quad 1.076958 \quad 0.879695$

H $4.468420 \quad-1.864918-3.155066$

H $\quad 3.481961 \quad-2.568039-1.853514$

H $\quad 5.183441 \quad-2.226387 \quad-1.568640$

$\begin{array}{lllll}H & 0.472821 & 0.976062 & -0.475717\end{array}$

H $\quad 4.138276 \quad 0.502287 \quad-3.453375$

H $\quad 3.641347 \quad 1.580013 \quad-2.031167$

C $\quad \begin{array}{llll}1.208501 & -0.885177 & -0.610331\end{array}$

Au $\quad-0.753693 \quad-1.361461 \quad-0.243667$

$\begin{array}{llll}\text { P } & -3.033639 & -1.914752 & 0.183404\end{array}$

H $\quad 1.525460 \quad-0.950448 \quad-1.651126$

C $\quad-4.204478-1.171373-1.026591$

H $\quad-5.245785 \quad-1.351221 \quad-0.740847$

H $\quad-4.009889-0.096967-1.077941$

H $\quad-4.021156-1.597471 \quad-2.016564$

C $\quad-3.607146 \quad-1.273435 \quad 1.811076$

$\begin{array}{llll}\text { H } & -3.051747 & -1.769485 & 2.611522\end{array}$

H $\quad-3.387914 \quad-0.203717 \quad 1.858752$

H $\quad-4.679056 \quad-1.443438 \quad 1.953880$

$\begin{array}{llll}\text { C } & -3.478576 & -3.702909 & 0.216562\end{array}$

H $\quad-2.880178-4.211760 \quad 0.976706$

H $\quad-4.540960 \quad-3.843922 \quad 0.439299$

H $\quad-3.250725 \quad-4.151783 \quad-0.753715$

O $\quad-2.243750 \quad 1.882699 \quad-1.103703$

$\begin{array}{llll}\mathrm{O} & -1.527994 & 1.776665 & 1.318372\end{array}$

S $\quad-1.286407 \quad 2.220078 \quad-0.053203$

$\begin{array}{lllll}0 & 0.221401 & 1.955004 & -0.506220\end{array}$ 


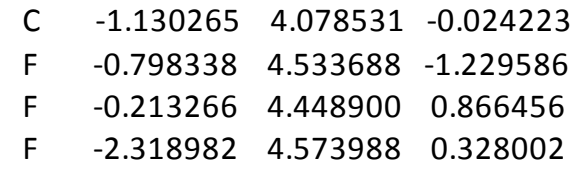

\begin{tabular}{|c|c|c|c|}
\hline & & & \\
\hline C & 4.033473 & -0.340318 & -1.545288 \\
\hline c & 4.612025 & 0.240918 & 0.810238 \\
\hline C & 3.655316 & -0.037485 & -0.120518 \\
\hline C & 2.226516 & -0.107758 & 0.241700 \\
\hline C & 1.866563 & 0.276706 & 1.663166 \\
\hline c & 2.935753 & -0.144828 & 2.669904 \\
\hline & 4.281528 & 0.459638 & 2.269806 \\
\hline C & 6.077237 & 0.347038 & 0.483139 \\
\hline C & 4.249627 & -1.799297 & -1.876769 \\
\hline & 4.169019 & 0.629749 & -2.455694 \\
\hline H & 0.883894 & -0.125672 & 1.919149 \\
\hline $\mathrm{H}$ & 1.763263 & 1.372362 & 1.688559 \\
\hline $\mathrm{H}$ & 2.656584 & 0.171885 & 3.680468 \\
\hline $\mathrm{H}$ & 3.017202 & -1.239446 & 2.686110 \\
\hline $\mathrm{H}$ & 5.093728 & 0.052092 & 2.885672 \\
\hline $\mathrm{H}$ & 4.277994 & 1.543468 & 2.468780 \\
\hline $\mathrm{H}$ & 6.642665 & -0.439937 & 1.000382 \\
\hline $\mathrm{H}$ & 6.279000 & 0.283403 & -0.585893 \\
\hline $\mathrm{H}$ & 6.476890 & 1.300896 & 0.852398 \\
\hline $\mathrm{H}$ & 4.472668 & -1.941666 & -2.938058 \\
\hline $\mathrm{H}$ & 3.362233 & -2.391653 & -1.625800 \\
\hline $\mathrm{H}$ & 5.079772 & -2.212691 & -1.290201 \\
\hline $\mathrm{H}$ & 0.428505 & 0.802307 & -0.623975 \\
\hline $\mathrm{H}$ & 4.438773 & 0.406921 & -3.484971 \\
\hline $\mathrm{H}$ & 4.005624 & 1.673659 & -2.205072 \\
\hline C & 1.241225 & -0.383 & -0.678 \\
\hline $\mathrm{Au}$ & -0.5 & $1-1.3$ & $5-0.2$ \\
\hline$P$ & -2.721608 & -2.191341 & 0.252697 \\
\hline $\mathrm{H}$ & 1.591945 & -0.539787 & -1.698776 \\
\hline C & -3.953541 & -1.636918 & -0.992384 \\
\hline $\mathrm{H}$ & -4.970092 & -1.904421 & -0.686729 \\
\hline $\mathrm{H}$ & -3.861650 & -0.552834 & -1.099376 \\
\hline $\mathrm{H}$ & -3.730536 & -2.100312 & -1.957094 \\
\hline C & -3.332289 & -1.505041 & 1.845088 \\
\hline $\mathrm{H}$ & -2.737314 & -1.912275 & 2.666899 \\
\hline $\mathrm{H}$ & -3.192942 & -0.420777 & 1.827258 \\
\hline $\mathrm{H}$ & -4.38 & -1.749744 & 2.003679 \\
\hline C & -2.97 & -4.01 & 0.392855 \\
\hline $\mathrm{H}$ & -2.326008 & -4.411772 & 1.174505 \\
\hline $\mathrm{H}$ & -4.017735 & -4.244245 & 0.634826 \\
\hline $\mathrm{H}$ & -2.710879 & -4.489397 & -0.553317 \\
\hline 0 & -2.554691 & 1.478475 & -1.018218 \\
\hline 0 & -1.616807 & 1.603055 & 1.311882 \\
\hline $\mathrm{S}$ & -1.532843 & 2.003015 & -0.101285 \\
\hline 0 & -0.097510 & 1.904602 & -0.666521 \\
\hline C & -1.723937 & 3.855636 & -0.130378 \\
\hline $\mathrm{F}$ & -1.601683 & 4.318324 & -1.376087 \\
\hline $\mathrm{F}$ & -0.800888 & 4.431227 & 0.645082 \\
\hline $\mathrm{F}$ & -2.939047 & 4.168317 & 0.334717 \\
\hline
\end{tabular}

Int3+TfO $\begin{array}{llll}\text { C } & -4.029573 & -0.405841 & -0.361174\end{array}$

$\begin{array}{llll}\text { C } & -3.056221 & -2.307148 & 0.931300\end{array}$

$\begin{array}{llll}\text { C } & -2.921001 & -1.381228 & -0.062483\end{array}$

$\begin{array}{llll}\text { C } & -1.691622 & -1.335627 & -0.868359\end{array}$

$\begin{array}{llll}\text { C } & -0.661911 & -2.417917 & -0.644880\end{array}$

$\begin{array}{llll}\text { C } & -0.572053 & -2.834908 & 0.821482\end{array}$

$\begin{array}{llll}\text { C } & -1.950586 & -3.259299 & 1.322373\end{array}$

$\begin{array}{llll}\text { C } & -4.329075 & -2.472193 & 1.715940\end{array}$

$\begin{array}{llll}\text { C } & -4.136250 & 0.797853 & 0.546943\end{array}$

$\begin{array}{llll}\text { C } & -4.875821 & -0.607959 & -1.377778\end{array}$

H $\quad 0.306771-2.094429-1.029769$

H $\quad-0.988128-3.277621 \quad-1.253315$

$\begin{array}{llll}H & 0.150288 & -3.648048 & 0.934566\end{array}$

$\begin{array}{llll}H & -0.184824 & -1.992384 & 1.403546\end{array}$

$\begin{array}{llll}H & -1.957687 & -3.376262 & 2.414046\end{array}$

$\begin{array}{llll}H & -2.212681 & -4.253821 & 0.924365\end{array}$

$\begin{array}{llll}H & -4.183004 & -2.141392 & 2.752953\end{array}$

H $\quad-5.168445 \quad-1.924082 \quad 1.287015$

$\begin{array}{llll}H & -4.599044 & -3.534088 & 1.770497\end{array}$

$\begin{array}{llll}H & -4.961080 & 1.451508 & 0.249002\end{array}$

$\begin{array}{llll}H & -3.206599 & 1.379931 & 0.529614\end{array}$

H $\quad-4.296173 \quad 0.492123 \quad 1.587928$

$\begin{array}{llll}H & -0.665609 & -0.581003 & -2.577493\end{array}$

$\begin{array}{lllll}H & -5.678893 & 0.091945 & -1.593584\end{array}$

$\begin{array}{llll}\text { H } & -4.795764 & -1.476798 & -2.024765\end{array}$

$\begin{array}{llll}\text { C } & -1.495535 & -0.416402 & -1.893867\end{array}$

$\begin{array}{llll}\mathrm{Au} & -0.454211 & 1.040422 & -0.502790\end{array}$

$\begin{array}{lllll}\text { P } & 0.540871 & 2.803634 & 0.675538\end{array}$

$\begin{array}{llll}\text { H } & -2.329016 & 0.178376 & -2.255057\end{array}$

$\begin{array}{llll}\text { C } & 2.103469 & 3.395408 & -0.076141\end{array}$

$\begin{array}{llll}\text { H } & 2.507975 & 4.226434 & 0.511057\end{array}$

$\begin{array}{llll}H & 2.823212 & 2.569924 & -0.103272\end{array}$

H $\quad 1.911449 \quad 3.731620-1.098349$

$\begin{array}{llll}\text { C } & 0.959544 & 2.350497 & 2.401337\end{array}$

$\begin{array}{llll}H & 0.040239 & 2.148536 & 2.957378\end{array}$

$\begin{array}{llll}H & 1.559979 & 1.437538 & 2.365043\end{array}$

H $\quad 1.511735 \quad 3.160877 \quad 2.887515$

$\begin{array}{lllll}\text { C } & -0.519457 & 4.303634 & 0.807741\end{array}$

$\begin{array}{llll}\text { H } & -1.453369 & 4.051197 & 1.316008\end{array}$

$\begin{array}{llll}H & -0.005654 & 5.091630 & 1.367478\end{array}$

$\begin{array}{lllll}H & -0.758928 & 4.672370 & -0.192841\end{array}$

$\begin{array}{lllll}\text { O } & 3.782871 & 0.555623 & -0.073849\end{array}$

$\begin{array}{llll}0 & 1.824701 & -0.453112 & 1.109155\end{array}$

S $\quad 2.700623-0.450311-0.097702$

$\begin{array}{llll}\text { O } & 1.950572 & -0.553018 & -1.380305\end{array}$

$\begin{array}{llll}\text { C } & 3.576367 & -2.087300 & 0.013181\end{array}$

F $\quad 4.427863 \quad-2.245528-1.007556$

$\begin{array}{llll}\text { F } & 2.681048 & -3.096849 & -0.027879\end{array}$

F $\quad 4.260656-2.185658 \quad 1.160695$

\section{TSC+TfO-}
$\begin{array}{llll}\text { C } & -2.379079 & 1.761164 & 0.108033\end{array}$
$\begin{array}{llll}\text { C } & -4.856817 & 1.080506 & -0.080464\end{array}$
$\begin{array}{llll}\text { C } & -3.532700 & 0.819325 & -0.101246\end{array}$
$\begin{array}{llll}\text { C } & -2.936145 & -0.493759 & -0.213672\end{array}$
$\begin{array}{llll}\text { C } & -3.812094 & -1.715284 & -0.220631\end{array}$
$\begin{array}{llll}\text { C } & -5.167361 & -1.399997 & 0.440399\end{array}$ 


$\begin{array}{cccc}\text { C } & -5.799497 & -0.111400 & -0.120006 \\ \text { C } & -5.473083 & 2.446129 & 0.024159 \\ \text { C } & -1.895203 & 2.005981 & 1.499836 \\ \text { C } & -2.040720 & 2.791482 & -0.922088 \\ \text { H } & -3.312409 & -2.555782 & 0.272762 \\ \text { H } & -3.980731 & -2.019202 & -1.263688 \\ \text { H } & -5.856547 & -2.240335 & 0.306928 \\ \text { H } & -5.021168 & -1.276149 & 1.520766 \\ \text { H } & -6.708743 & 0.130368 & 0.444548 \\ \text { H } & -6.127787 & -0.274564 & -1.158480 \\ \text { H } & -6.044498 & 2.544878 & 0.956638 \\ \text { H } & -4.737064 & 3.252659 & -0.006478 \\ \text { H } & -6.186329 & 2.609693 & -0.793970 \\ \text { H } & -0.809854 & 2.176721 & 1.529720 \\ \text { H } & -2.167522 & 1.194201 & 2.174962 \\ \text { H } & -2.376842 & 2.932419 & 1.850363 \\ \text { H } & -0.949841 & 2.890837 & -0.997231 \\ \text { H } & -2.430958 & 3.760634 & -0.578620 \\ \text { H } & -2.469278 & 2.557568 & -1.897642 \\ \text { C } & -1.588297 & -0.399840 & -0.291002 \\ \text { Au } & 0.118670 & -1.505347 & -0.135502 \\ \text { P } & 2.193790 & -2.635544 & 0.081130 \\ \text { H } & -1.235975 & 0.679099 & -0.458015 \\ \text { C } & 3.031581 & -2.269835 & 1.672883 \\ \text { H } & 4.004824 & -2.768488 & 1.725000 \\ \text { H } & 3.158575 & -1.185804 & 1.728373 \\ \text { H } & 2.405527 & -2.604032 & 2.504083 \\ \text { C } & 3.402912 & -2.141750 & -1.208245 \\ \text { H } & 3.011463 & -2.395165 & -2.196731 \\ \text { H } & 3.525279 & -1.058311 & -1.134485 \\ \text { H } & 4.363879 & -2.643516 & -1.055692 \\ \text { C } & 2.128147 & -4.475960 & -0.007234 \\ \text { H } & 1.708765 & -4.782138 & -0.969162 \\ \text { H } & 3.126863 & -4.909874 & 0.103888 \\ \text { H } & 1.480646 & -4.857156 & 0.786708 \\ \text { O } & 1.335193 & 2.211603 & 1.586927 \\ \text { O } & 2.805734 & 0.641833 & 0.299817 \\ \text { S } & 1.886733 & 1.807842 & 0.273209 \\ \text { O } & 0.885466 & 1.779835 & -0.829754 \\ \text { C } & 2.997631 & 3.217564 & -0.208178 \\ \text { F } & 2.290231 & 4.356443 & -0.305566 \\ \text { F } & 3.576980 & 2.981287 & -1.395362 \\ \text { F } & 3.963166 & 3.397759 & 0.704333\end{array}$

Int4+TfO-

$\begin{array}{lllll}\text { C } & & -2.37201 & 1.61461 & -0.07146 \\ \text { C } & & -4.91624 & 0.97794 & -0.03739 \\ \text { C } & & -3.59934 & 0.70872 & -0.09339 \\ \text { C } & & -3.04255 & -0.62684 & -0.16594\end{array}$

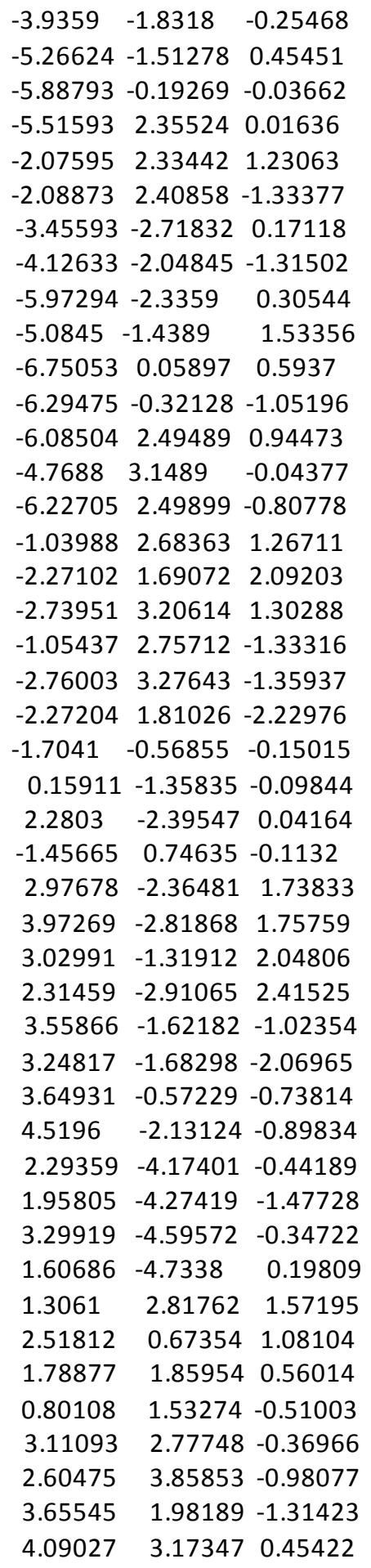

xyz for Ag-triflate shuttle of scheme 9C at B3LYP/LAN2DZ(Au), 6-31G(d,p) (C, H, O, S, P, F) level 


\begin{tabular}{|c|c|c|c|c|c|c|c|}
\hline C & -2.686364 & -0.943312 & 0.026320 & $\mathrm{H}$ & -5.392577 & -3.100960 & 0.354235 \\
\hline C & -3.378826 & -2.279759 & 0.256044 & $\mathrm{H}$ & -5.107635 & -1.769002 & 1.469837 \\
\hline C & -4.892049 & -2.132364 & 0.456600 & $\mathrm{H}$ & -6.567718 & -1.053635 & -0.447904 \\
\hline C & -5.790060 & 1.405314 & -0.507541 & $\mathrm{H}$ & -5.290251 & -1.492584 & -1.577774 \\
\hline C & -2.872446 & 1.485516 & 0.398236 & $\mathrm{H}$ & -6.586614 & 1.304194 & 0.241685 \\
\hline C & -2.754967 & 2.681743 & -0.449372 & $\mathrm{H}$ & -5.322989 & 2.379451 & -0.369835 \\
\hline C & -1.355991 & -0.793775 & -0.190167 & $\mathrm{H}$ & -6.291334 & 1.393513 & -1.484070 \\
\hline $\mathrm{Ag}$ & 0.451629 & -1.870605 & -0.094495 & $\mathrm{H}$ & -1.161303 & 1.765983 & 1.651398 \\
\hline$P$ & 2.816364 & -2.564251 & -0.012623 & $\mathrm{H}$ & -2.378834 & 0.632010 & 2.310265 \\
\hline C & 3.345060 & -4.335073 & 0.055343 & $\mathrm{H}$ & -2.667853 & 2.411052 & 2.274524 \\
\hline C & -2.245006 & 1.548807 & 1.737916 & $\mathrm{H}$ & -1.686009 & 2.641054 & -0.777468 \\
\hline C & 3.679504 & -1.791797 & 1.420651 & $\mathrm{H}$ & -2.847452 & 3.620319 & 0.106966 \\
\hline C & 3.735703 & -1.884223 & -1.458040 & $\mathrm{H}$ & -3.377505 & 2.665102 & -1.343512 \\
\hline 0 & 0.824479 & 2.096142 & 1.456968 & $\mathrm{H}$ & -1.070714 & 0.225413 & -0.477853 \\
\hline$S$ & 1.313356 & 1.941363 & 0.064023 & $\mathrm{H}$ & 4.758418 & -1.975679 & 1.383583 \\
\hline C & 2.541642 & 3.322774 & -0.129524 & $\mathrm{H}$ & 3.482192 & -0.717556 & 1.379159 \\
\hline $\mathrm{F}$ & 3.526248 & 3.206229 & 0.774352 & $\mathrm{H}$ & 3.277230 & -2.188196 & 2.356845 \\
\hline 0 & 2.126328 & 0.723763 & -0.187671 & $\mathrm{H}$ & 3.378629 & -2.351141 & -2.380044 \\
\hline $\mathrm{O}$ & 0.292093 & 2.237756 & -0.977632 & $\mathrm{H}$ & 3.526965 & -0.812290 & -1.502013 \\
\hline $\mathrm{F}$ & 1.944125 & 4.511466 & 0.046735 & $\mathrm{H}$ & 4.813583 & -2.051407 & -1.361690 \\
\hline$F$ & 3.088078 & 3.302083 & -1.354116 & $\mathrm{H}$ & 2.967399 & -4.865372 & -0.823324 \\
\hline $\mathrm{H}$ & -2.925675 & -2.791167 & 1.113448 & $\mathrm{H}$ & 4.435852 & -4.426524 & 0.085071 \\
\hline $\mathrm{H}$ & -3.191281 & -2.919933 & -0.615818 & $\mathrm{H}$ & 2.924199 & -4.809988 & 0.94592 \\
\hline
\end{tabular}

ISb + TfO-
C $\quad-5.297546 \quad-1.741985 \quad 0.130262$
C $\quad-4.801110 \quad-0.335112 \quad-0.133885$
C $\quad-3.460945 \quad-0.099774 \quad-0.132993$
C $\quad-2.436575 \quad-1.161450 \quad 0.007171$
C $\quad-2.931438 \quad-2.533052 \quad 0.452241$
C $\quad-4.338842 \quad-2.508472 \quad 1.053607$
C $\quad \begin{array}{llll}-5.869091 & 0.720971 & -0.255644\end{array}$

C $\quad-2.971361 \quad 1.302914 \quad-0.161043$

C $\quad-3.015265 \quad 2.059926 \quad-1.444314$

C $\quad-1.153106 \quad-0.921840 \quad-0.341082$

Ag $\quad 0.706633 \quad-1.876944 \quad-0.176245$

$\begin{array}{llll}\text { P } & 3.125045 & -2.342627 & -0.022175\end{array}$

C $\quad 3.813658-4.057565 \quad 0.034088$

C $-2.402403 \quad 1.899655 \quad 0.979455$ 


\begin{tabular}{|c|c|c|c|c|c|c|c|}
\hline C & 3.879742 & -1.524287 & 1.448615 & $\mathrm{H}$ & -5.488133 & 1.717092 & -0.491602 \\
\hline C & 4.026341 & -1.557805 & -1.426785 & $\mathrm{H}$ & -6.595127 & 0.444311 & -1.031228 \\
\hline $\mathrm{O}$ & 0.227083 & 1.796028 & 1.103777 & $\mathrm{H}$ & -1.147707 & 1.778632 & 0.890452 \\
\hline$S$ & 1.094644 & 2.045615 & -0.125844 & $\mathrm{H}$ & -2.591744 & 1.400680 & 1.929527 \\
\hline C & 1.851384 & 3.693705 & 0.285550 & $\mathrm{H}$ & -2.453463 & 2.989772 & 1.012367 \\
\hline $\mathrm{F}$ & 2.563058 & 3.614344 & 1.415141 & $\mathrm{H}$ & -2.002971 & 1.969754 & -1.873604 \\
\hline $\mathrm{O}$ & 2.238533 & 1.122707 & -0.215567 & $\mathrm{H}$ & -3.195021 & 3.128979 & -1.299316 \\
\hline $\mathrm{O}$ & 0.295489 & 2.283364 & -1.339950 & $\mathrm{H}$ & -3.722208 & 1.630659 & -2.155426 \\
\hline $\mathrm{F}$ & 0.887558 & 4.611277 & 0.447035 & $\mathrm{H}$ & -0.977722 & 0.074387 & -0.753539 \\
\hline $\mathrm{F}$ & 2.657216 & 4.085988 & -0.707503 & $\mathrm{H}$ & 4.970417 & -1.623133 & 1.445836 \\
\hline $\mathrm{H}$ & -2.209606 & -2.957656 & 1.158678 & $\mathrm{H}$ & 3.605209 & -0.466612 & 1.421433 \\
\hline $\mathrm{H}$ & -2.935287 & -3.200342 & -0.421004 & $\mathrm{H}$ & 3.480228 & -1.965170 & 2.365868 \\
\hline $\mathrm{H}$ & -4.703400 & -3.528426 & 1.219190 & $\mathrm{H}$ & 3.735959 & -2.035170 & -2.366648 \\
\hline $\mathrm{H}$ & -4.321631 & -2.011222 & 2.032278 & $\mathrm{H}$ & 3.734156 & -0.505225 & -1.464854 \\
\hline $\mathrm{H}$ & -6.301964 & -1.695644 & 0.569254 & $\mathrm{H}$ & 5.111284 & -1.637325 & -1.301483 \\
\hline $\mathrm{H}$ & -5.406683 & -2.284183 & -0.822099 & $\mathrm{H}$ & 3.509288 & -4.604243 & -0.862683 \\
\hline $\mathrm{H}$ & -6.437562 & 0.802129 & 0.681190 & & & & \\
\hline $\mathrm{H}$ & 4.906958 & -4.047496 & 0.092355 & C & 1.870127 & 1.372731 & -1.613071 \\
\hline $\mathrm{H}$ & 3.414986 & -4.584870 & 0.905122 & $\mathrm{Ag}$ & -0.270857 & 0.974065 & -0.491754 \\
\hline Int & 3+TfO- & & & $P$ & -2.369885 & 2.257088 & -0.059831 \\
\hline C & 4.095877 & -2.014425 & -0.238176 & C & -2.466171 & 4.074218 & -0.385149 \\
\hline C & 4.016265 & -0.735176 & 0.565640 & C & 4.080122 & 2.591776 & 0.768790 \\
\hline C & 3.261291 & 0.327229 & 0.177084 & C & -2.966422 & 2.107948 & 1.681149 \\
\hline C & 2.446835 & 0.264375 & -1.055119 & $\mathrm{C}$ & -3.766557 & 1.563107 & -1.041813 \\
\hline C & 2.310860 & -1.090662 & -1.716561 & $\mathrm{O}$ & -0.574251 & -3.289410 & -0.631575 \\
\hline C & 3.643411 & -1.844705 & -1.688519 & $S$ & -1.149877 & -1.993468 & -0.255896 \\
\hline C & 4.839896 & -0.740247 & 1.825322 & $\mathrm{C}$ & -2.643240 & -2.406505 & 0.773573 \\
\hline C & 3.222037 & 1.590117 & 0.994611 & $\mathrm{~F}$ & -2.291825 & -3.010927 & 1.912146 \\
\hline C & 2.174281 & 1.660685 & 2.081912 & 0 & -0.313165 & -1.172095 & 0.684489 \\
\hline
\end{tabular}



H $\quad-3.696752 \quad 2.764950 \quad-1.578205$
H $\quad 3.826809 \quad-1.490469-2.004332$
H $\quad-2.559996 \quad 1.603417 \quad-2.281924$
H $\quad 4.029345 \quad-0.378924 \quad-0.639034$
H $\quad-1.655641 \quad 1.094493 \quad-0.042811$
H $\quad 5.045234 \quad-1.847295 \quad-0.751228$
H $\quad 4.445208 \quad-2.646173 \quad 1.789440$
H $\quad 2.731807 \quad-4.198756-1.559093$
$\begin{array}{llll}\text { H } & 3.337247 & -1.270307 & 2.102071\end{array}$
H $\quad 4.044146 \quad-4.411730 \quad-0.373895$
$\begin{array}{llll}H & 2.788215 & -2.936158 & 2.384931\end{array}$
H $\quad 2.354388 \quad-4.744676 \quad 0.082987$ 


\section{References}

1) Block, B. P.; Bailar, J. C., Jr. J. Am. Chem. Soc. 1951, 73, 4722.

2) de Frémont, P.; Scott, N. M.; Stevens, E. D.; Nolan, S. P. Organometallics 2005, 24, 2411.

3) (a) Cadran, N.; Cariou, K.; Hervé, G.; Fensterbank, L.; Malacria, M.; Marco-Contelles, J. J. Am. Chem. Soc. 2004 , 126 , 3408; (b) Lemière, G.; Gandon, V.; Agenet, N.; Goddard, J.-P.; de Kozak, A.; Aubert, C.; Fensterbank, L.; Malacria, M. Angew. Chem. Int. Ed. 2006, 45, 7596.

4) Cheong, P. H.-Y.; Morganelli, P.; Luzung, M. R.; Houk, K. N.; Toste, F. D. J. Am. Chem. Soc. $2008,130,4517$.

5) Santos, L. S.; Metzger, J. O. Rapid Comm. Mass Spectrom. 2008, 22, 898.

6) Gaussian 03, Revision C.02, Frisch, M. J.; Trucks, G. W.; Schlegel, H. B.; Scuseria, G. E.; Robb, M. A.; Cheeseman, J. R.; Montgomery, Jr., J. A.; Vreven, T.; Kudin, K. N.; Burant, J. C.; Millam, J. M.; Iyengar, S. S.; Tomasi, J.; Barone, V.; Mennucci, B.; Cossi, M.; Scalmani, G.; Rega, N.; Petersson, G. A.; Nakatsuji, H.; Hada, M.; Ehara, M.; Toyota, K.; Fukuda, R.; Hasegawa, J.; Ishida, M.; Nakajima, T.; Honda, Y.; Kitao, O.; Nakai, H.; Klene, M.; Li, X.; Knox, J. E.; Hratchian, H. P.; Cross, J. B.; Bakken, V.; Adamo, C.; Jaramillo, J.; Gomperts, R.; Stratmann, R. E.; Yazyev, O.; Austin, A. J.; Cammi, R.; Pomelli, C.; Ochterski, J. W.; Ayala, P. Y.; Morokuma, K.; Voth, G. A.; Salvador, P.; Dannenberg, J. J.; Zakrzewski, V. G.; Dapprich, S.; Daniels, A. D.; Strain, M. C.; Farkas, O.; Malick, D. K.; Rabuck, A. D.; Raghavachari, K.; Foresman, J. B.; Ortiz, J. V.; Cui, Q.; Baboul, A. G.; Clifford, S.; Cioslowski, J.; Stefanov, B. B.; Liu, G.; Liashenko, A.; Piskorz, P.; Komaromi, I.; Martin, R. L.; Fox, D. J.; Keith, T.; Al-Laham, M. A.; Peng, C. Y.; Nanayakkara, A.; Challacombe, M.; Gill, P. M. W.; Johnson, B.; Chen, W.; Wong, M. W.; Gonzalez, C.; and Pople, J. A.; Gaussian, Inc., Wallingford CT, 2004. 
9. NMR Spectra 
${ }^{1} \mathrm{H}$ NMR (400 MHz, $\mathrm{CDCl}_{3}$ )

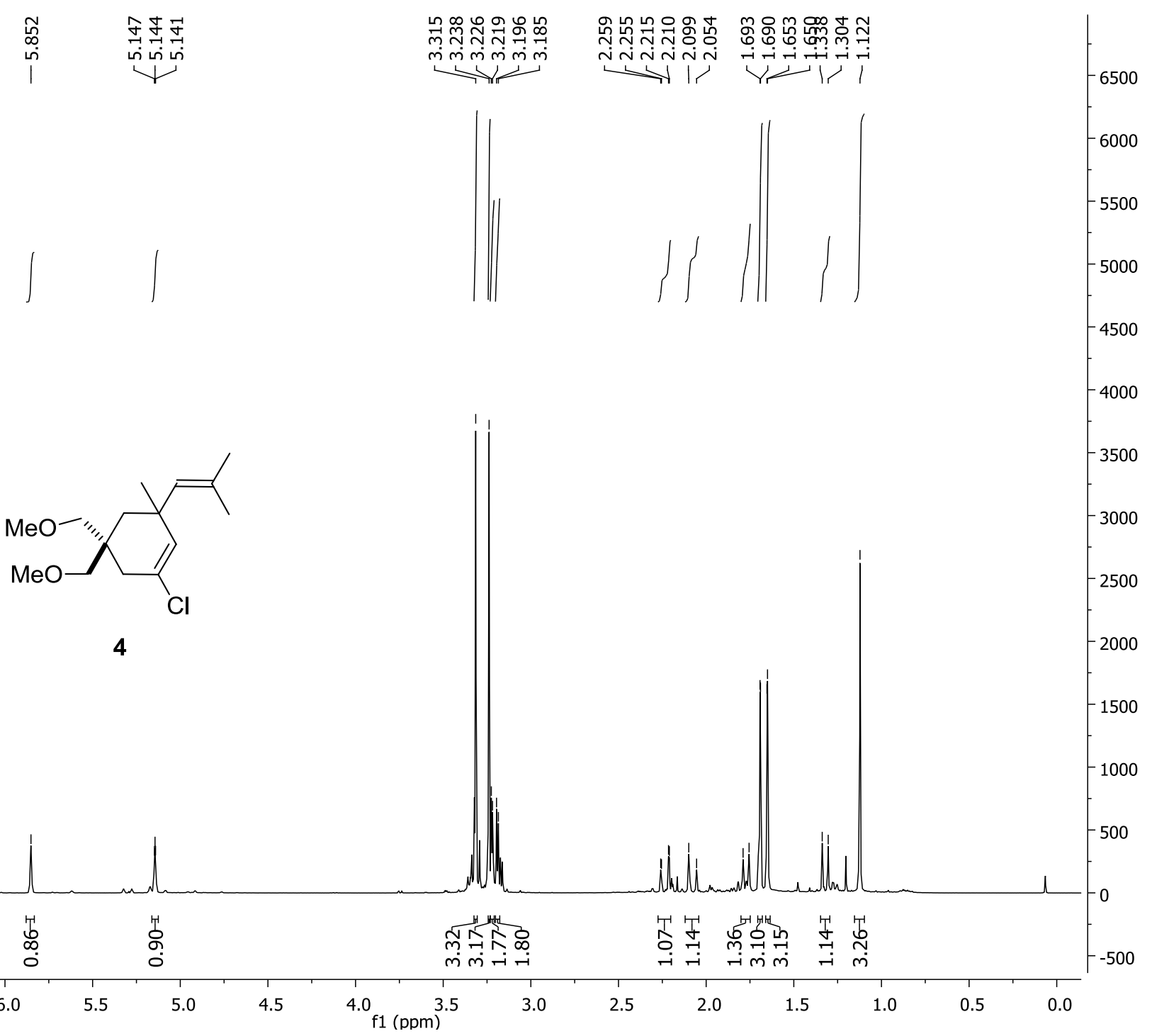


${ }^{13} \mathrm{C} \mathrm{NMR}\left(100 \mathrm{MHz}, \mathrm{CDCl}_{3}\right)$

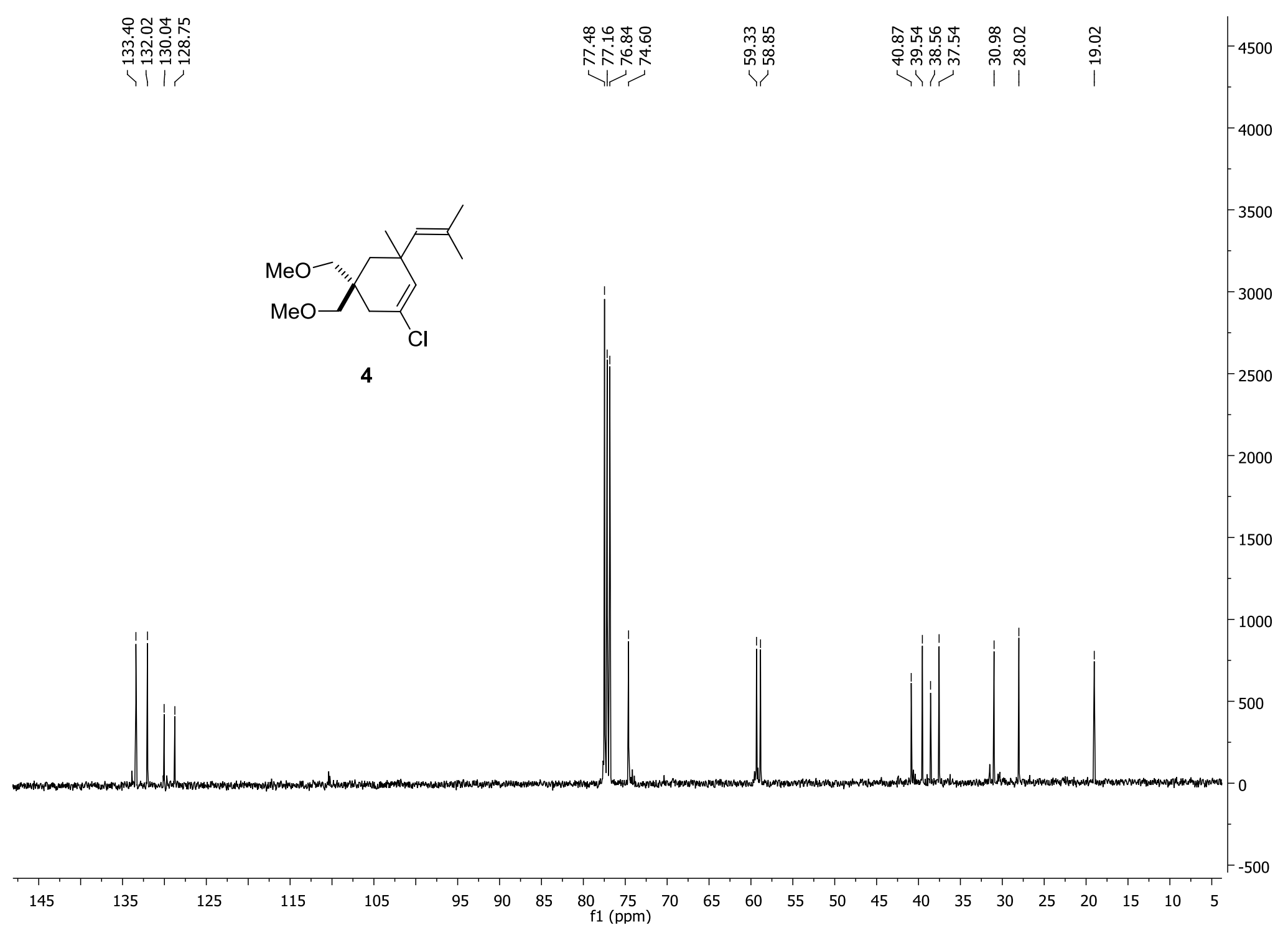


${ }^{1} \mathrm{H}$ NMR (500 MHz, $\mathrm{CDCl}_{3}$ )

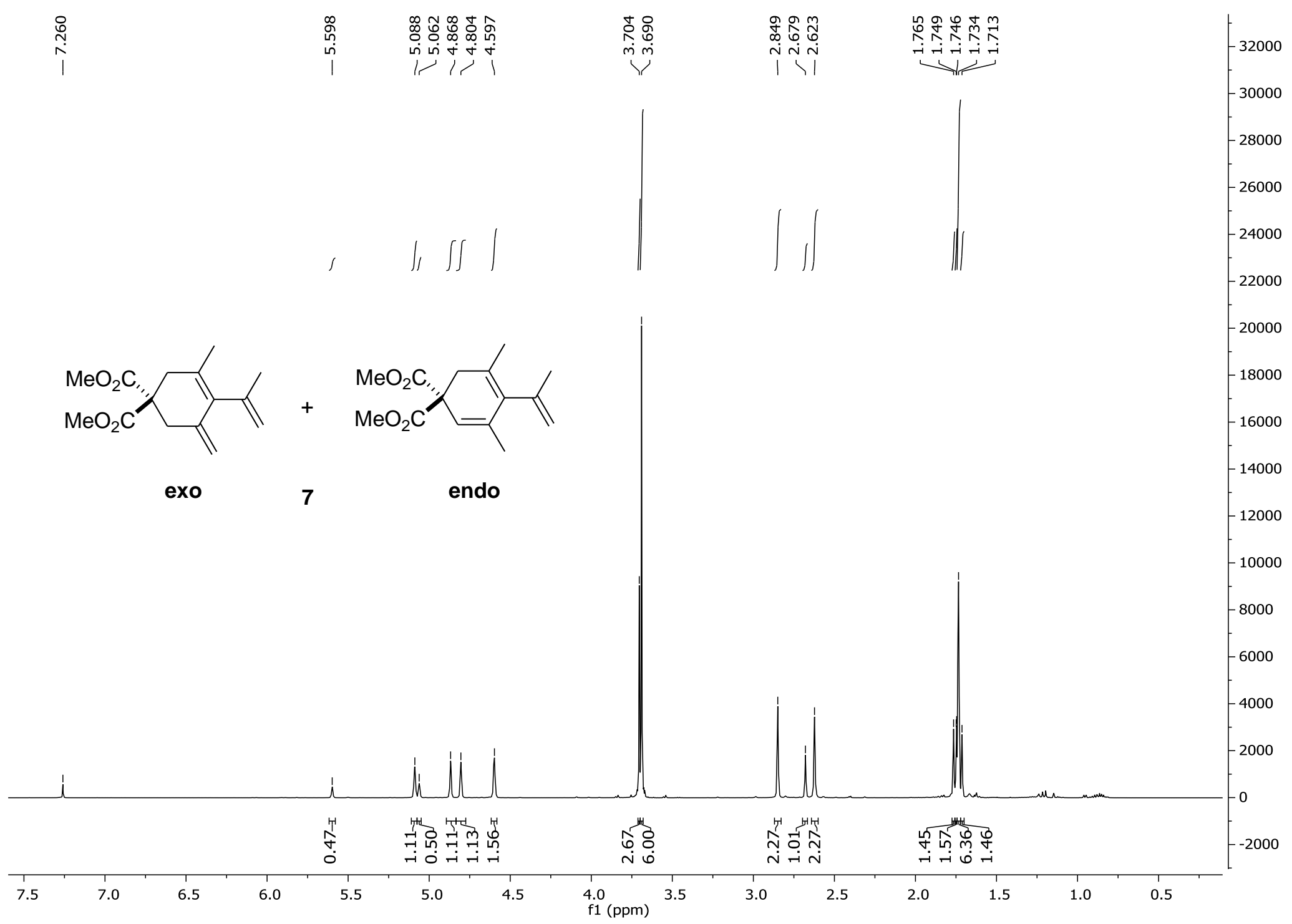


${ }^{13} \mathrm{C}$ NMR (125 MHz, $\left.\mathrm{CDCl}_{3}\right)$

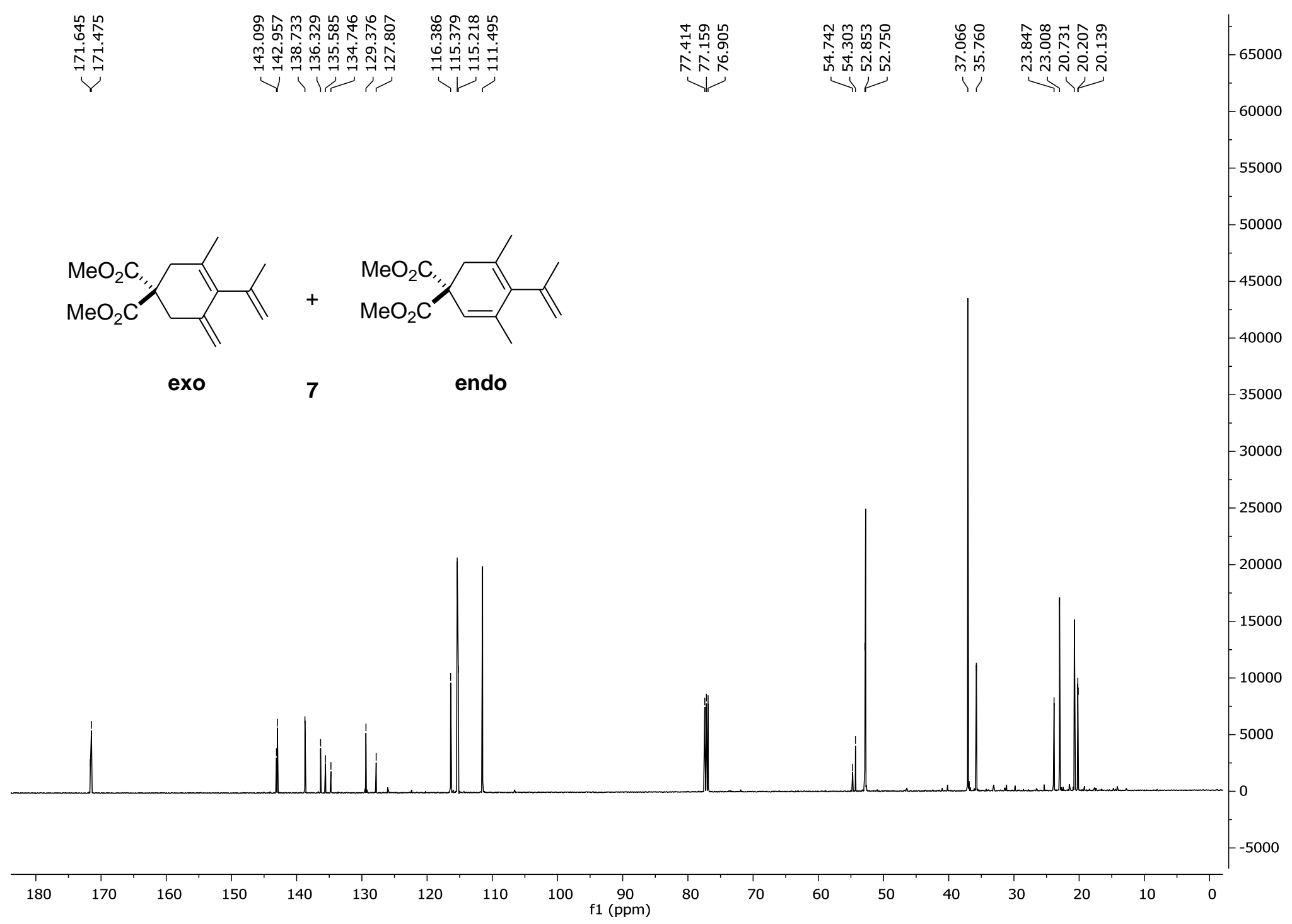


${ }^{1} \mathrm{H}$ NMR (600 MHz, $\mathrm{CDCl}_{3}$ )

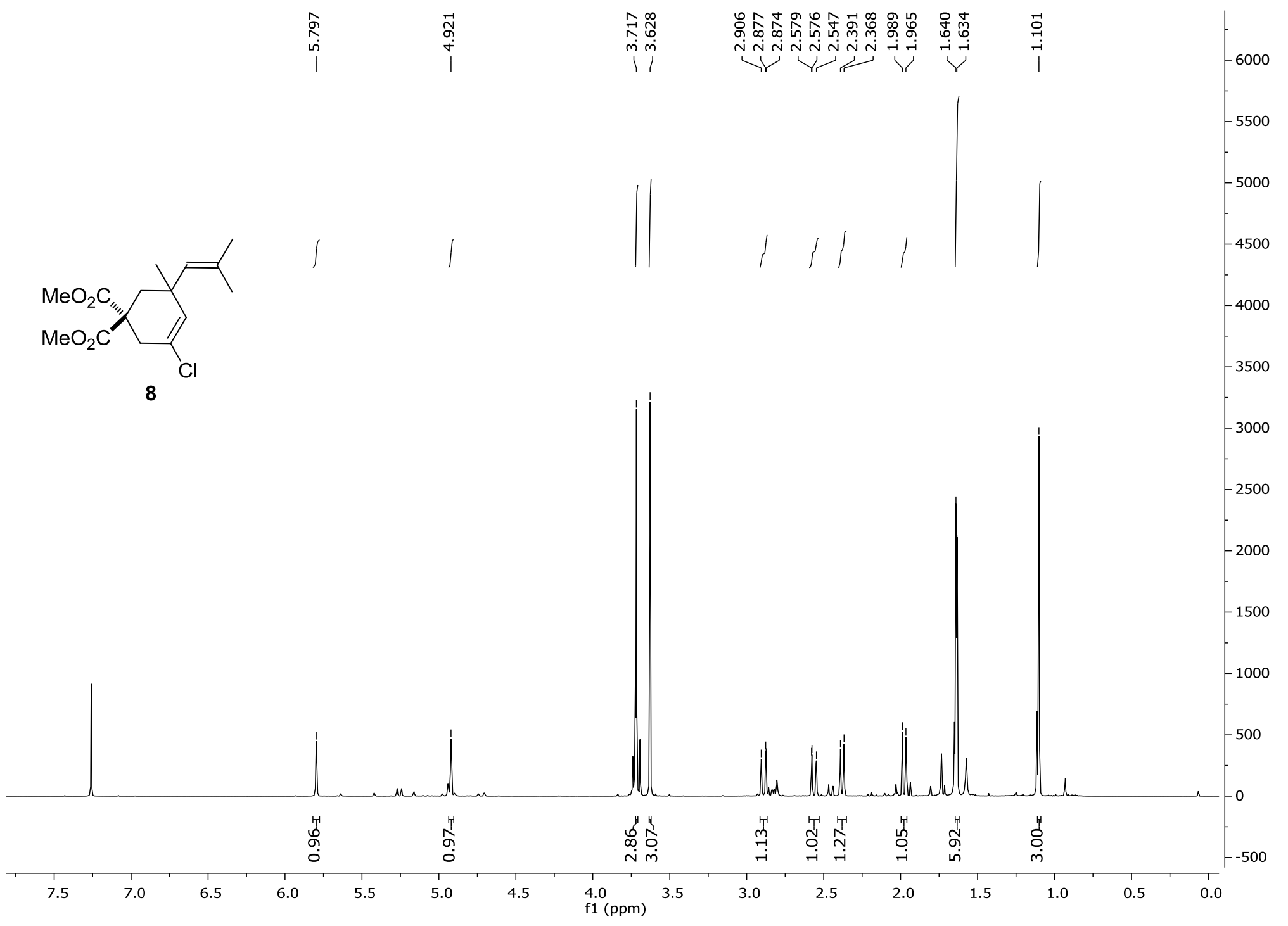


${ }^{13} \mathrm{C} \mathrm{NMR}\left(150 \mathrm{MHz}, \mathrm{CDCl}_{3}\right)$

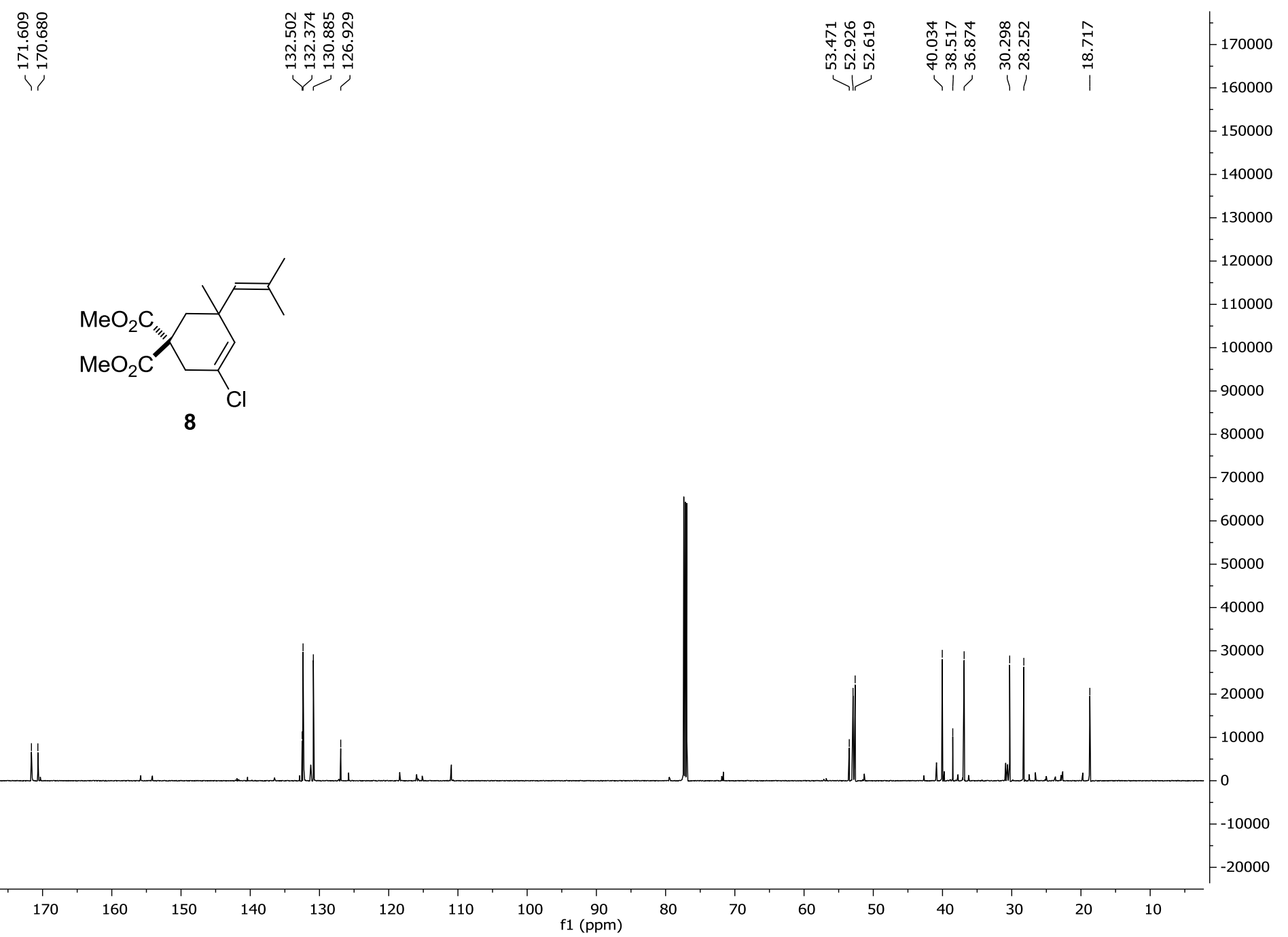


${ }^{1} \mathrm{H}$ NMR $\left(250 \mathrm{MHz}, \mathrm{CDCl}_{3}\right)$

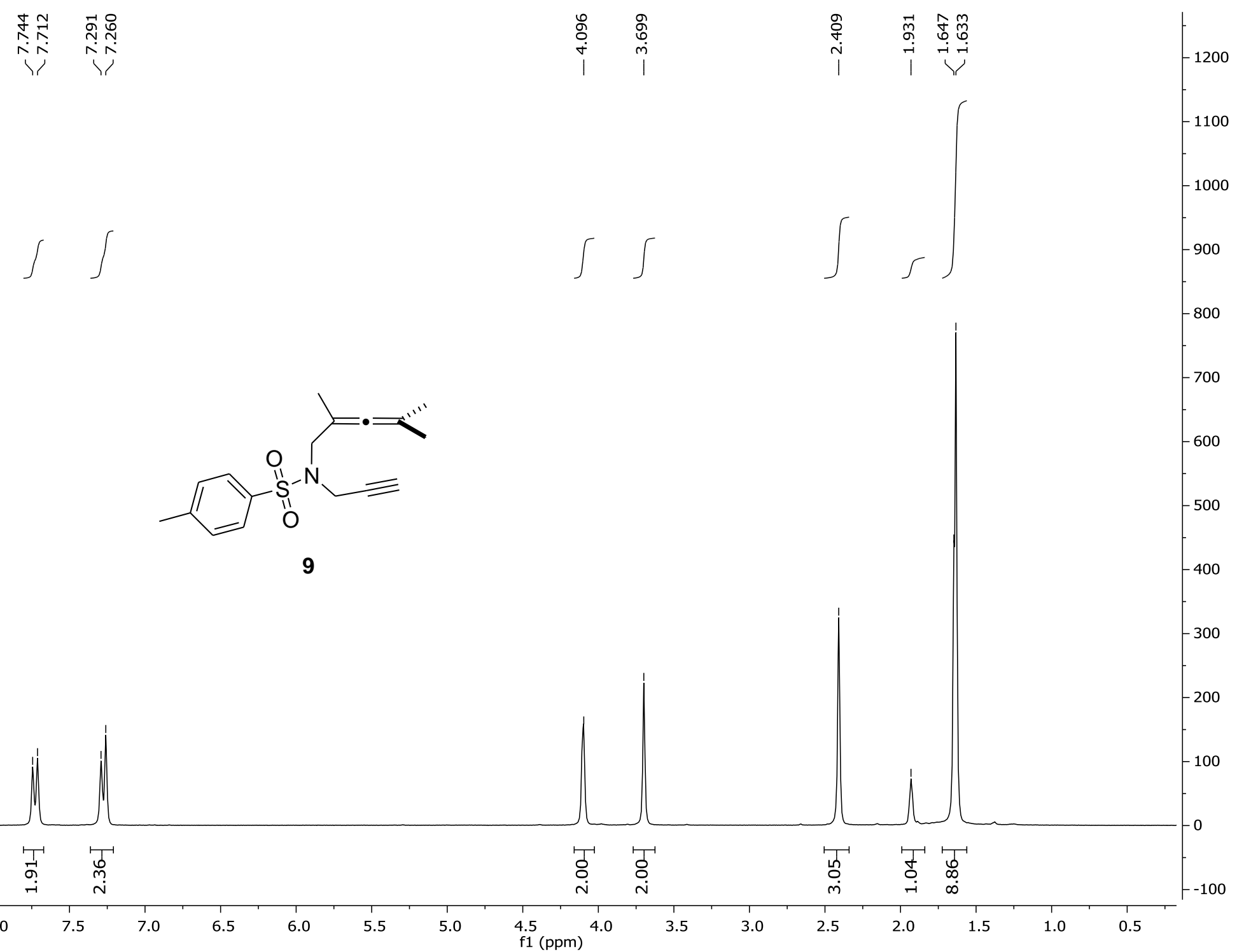


${ }^{13} \mathrm{C}$ NMR (62.5 MHz, $\mathrm{CDCl}_{3}$ )
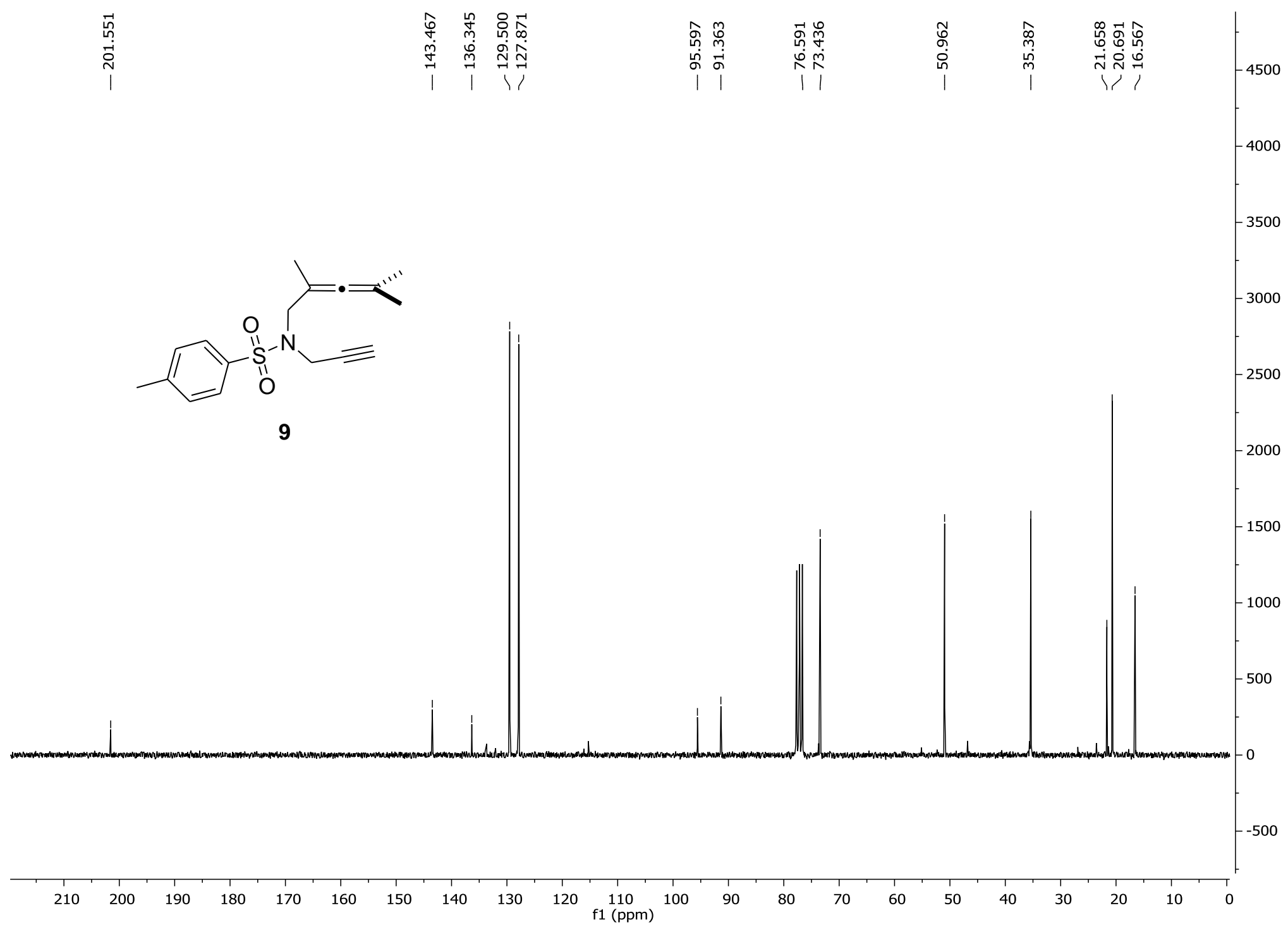
${ }^{1} \mathrm{H}\left(250 \mathrm{MHz}, \mathrm{CDCl}_{3}\right)$

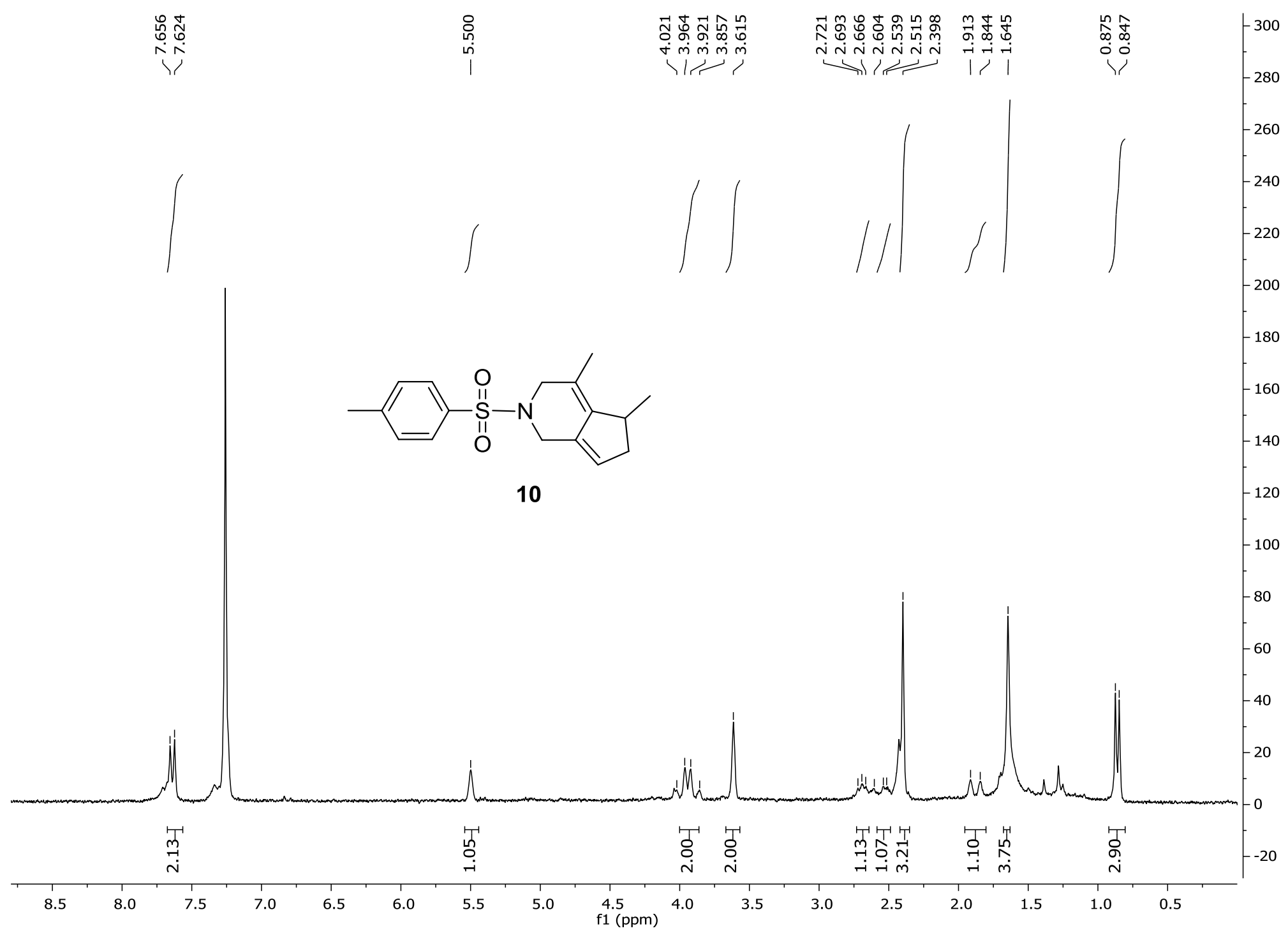


${ }^{13} \mathrm{C}$ NMR (125 MHz, $\left.\mathrm{CDCl}_{3}\right)$

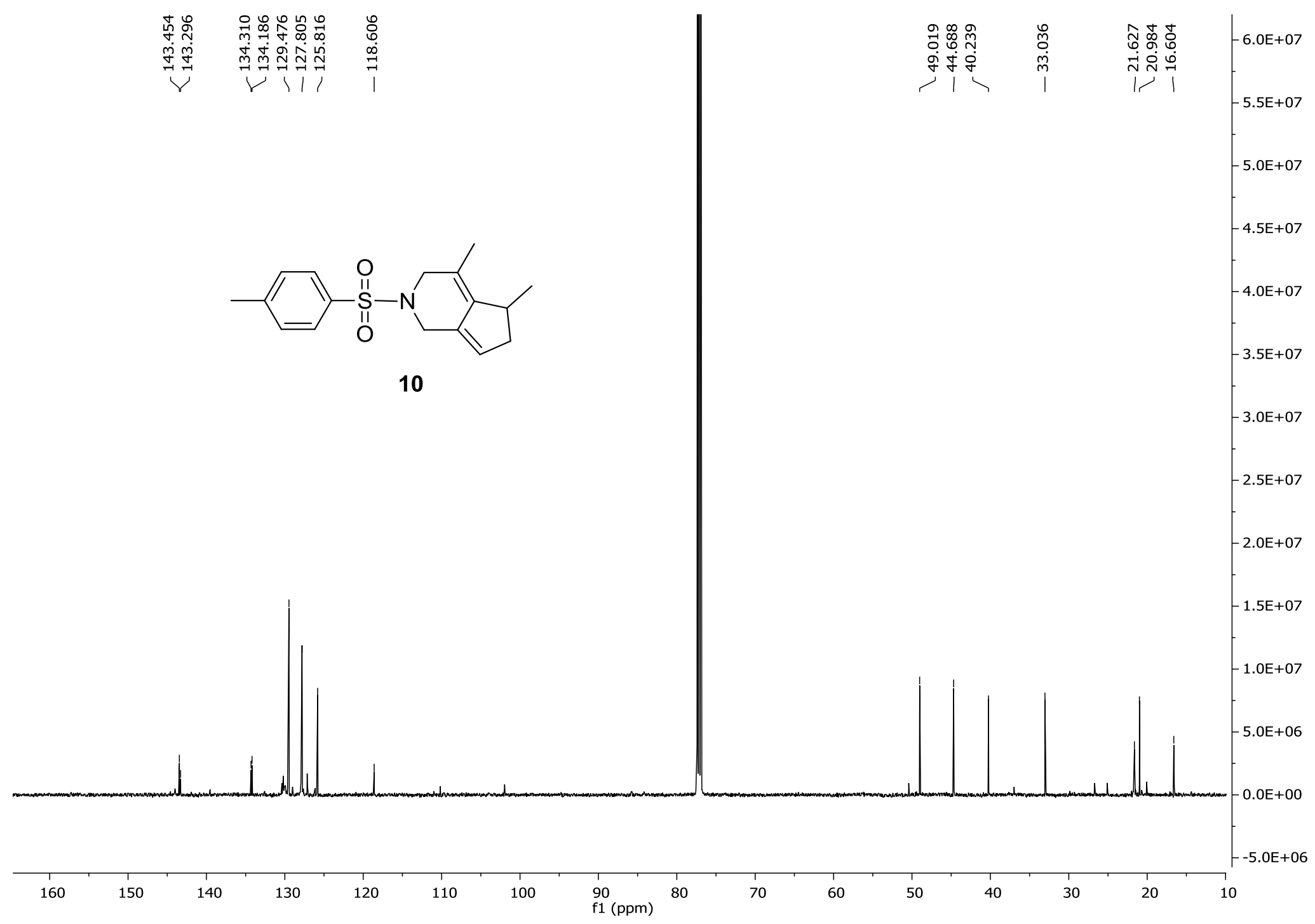


${ }^{1} \mathrm{H}$ NMR (400 MHz, $\mathrm{CDCl}_{3}$ )

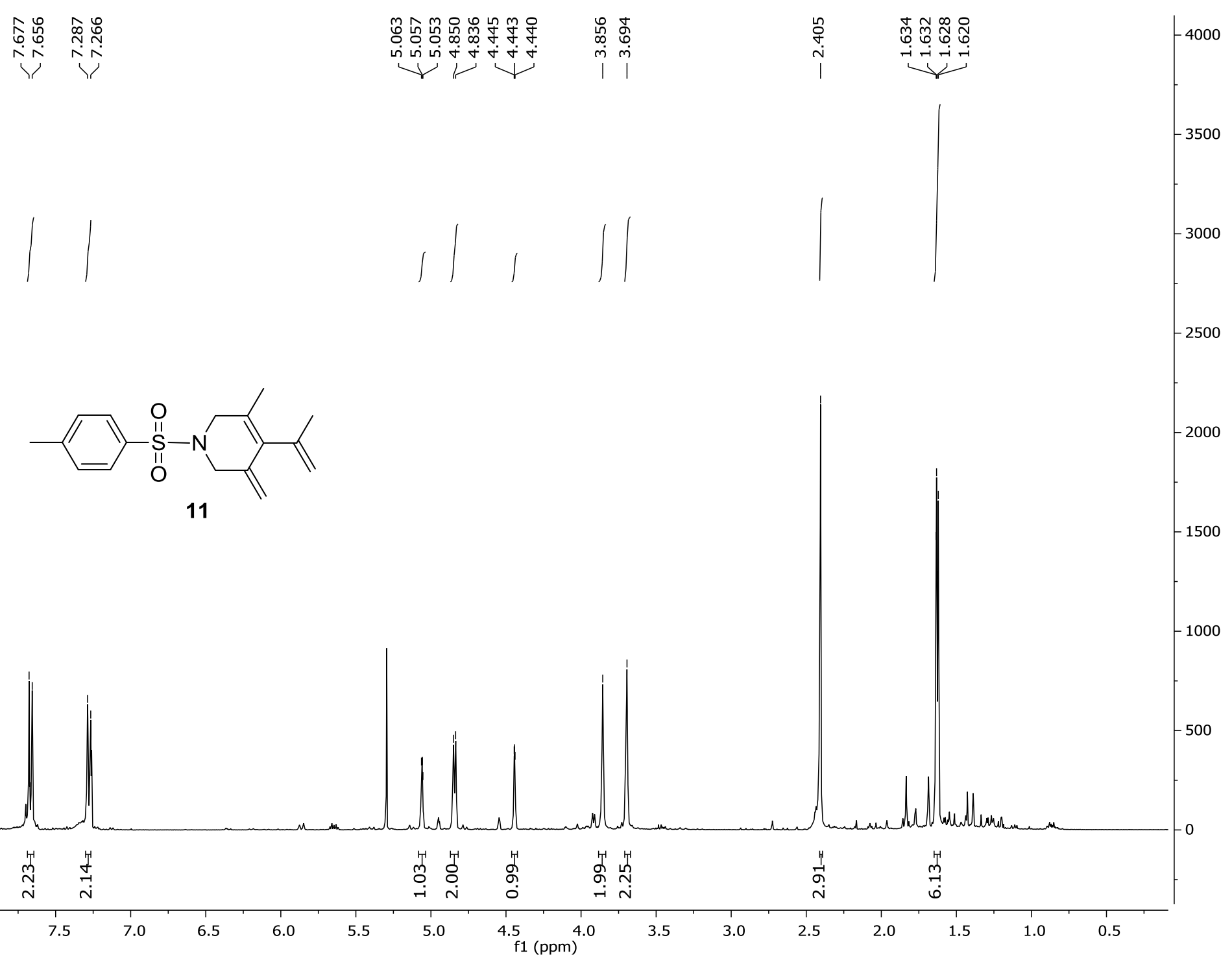


${ }^{13} \mathrm{C}$ NMR $\left(100 \mathrm{MHz}, \mathrm{CDCl}_{3}\right)$

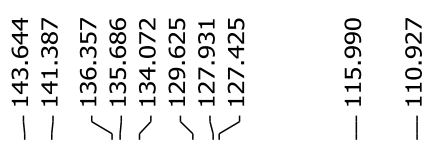

余早

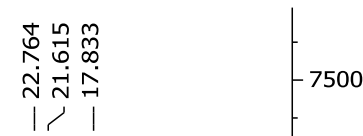

7000

6500

6000

5500
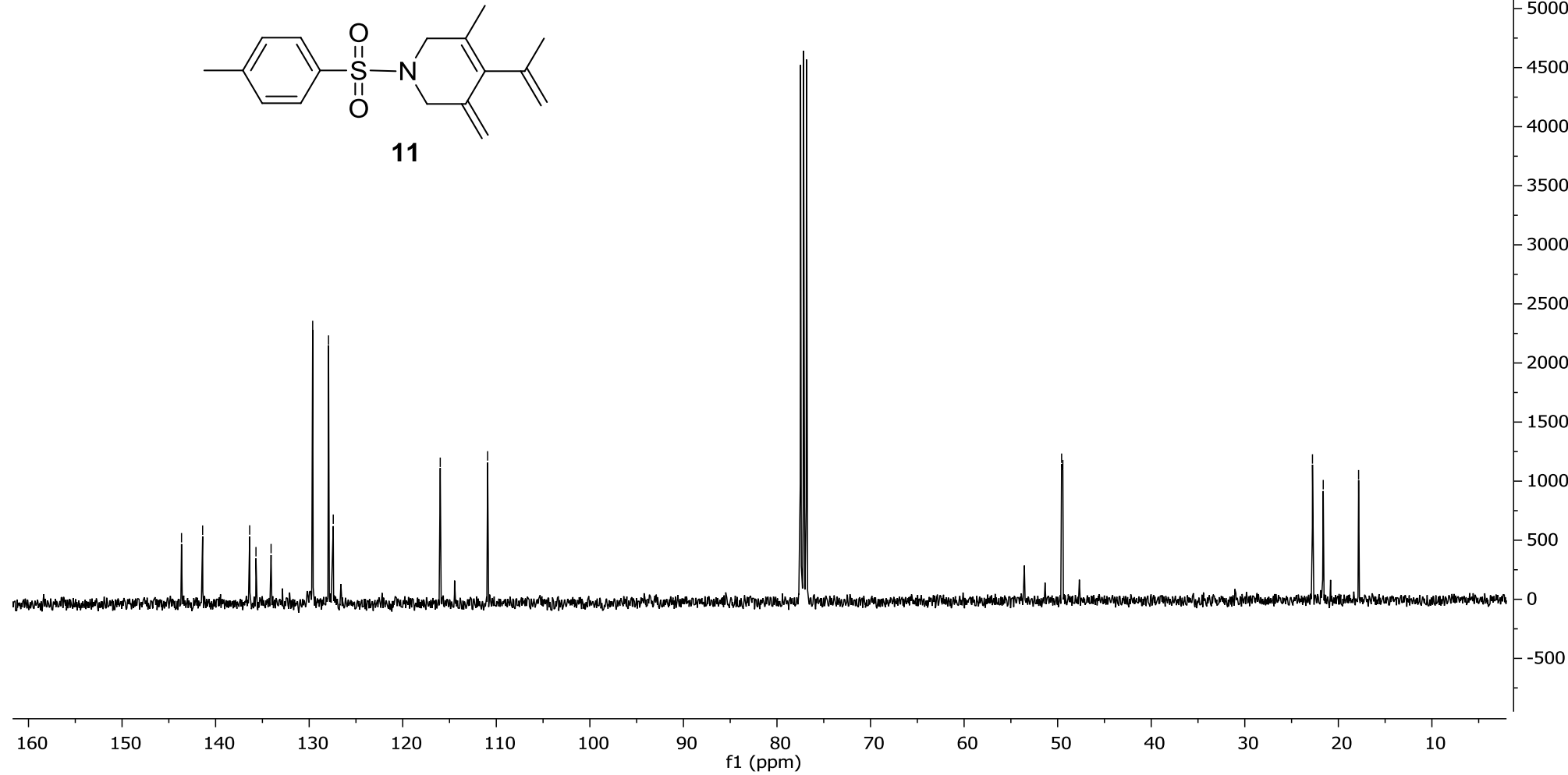
${ }^{1} \mathrm{H}$ NMR (500 MHz, $\mathrm{CDCl}_{3}$ )

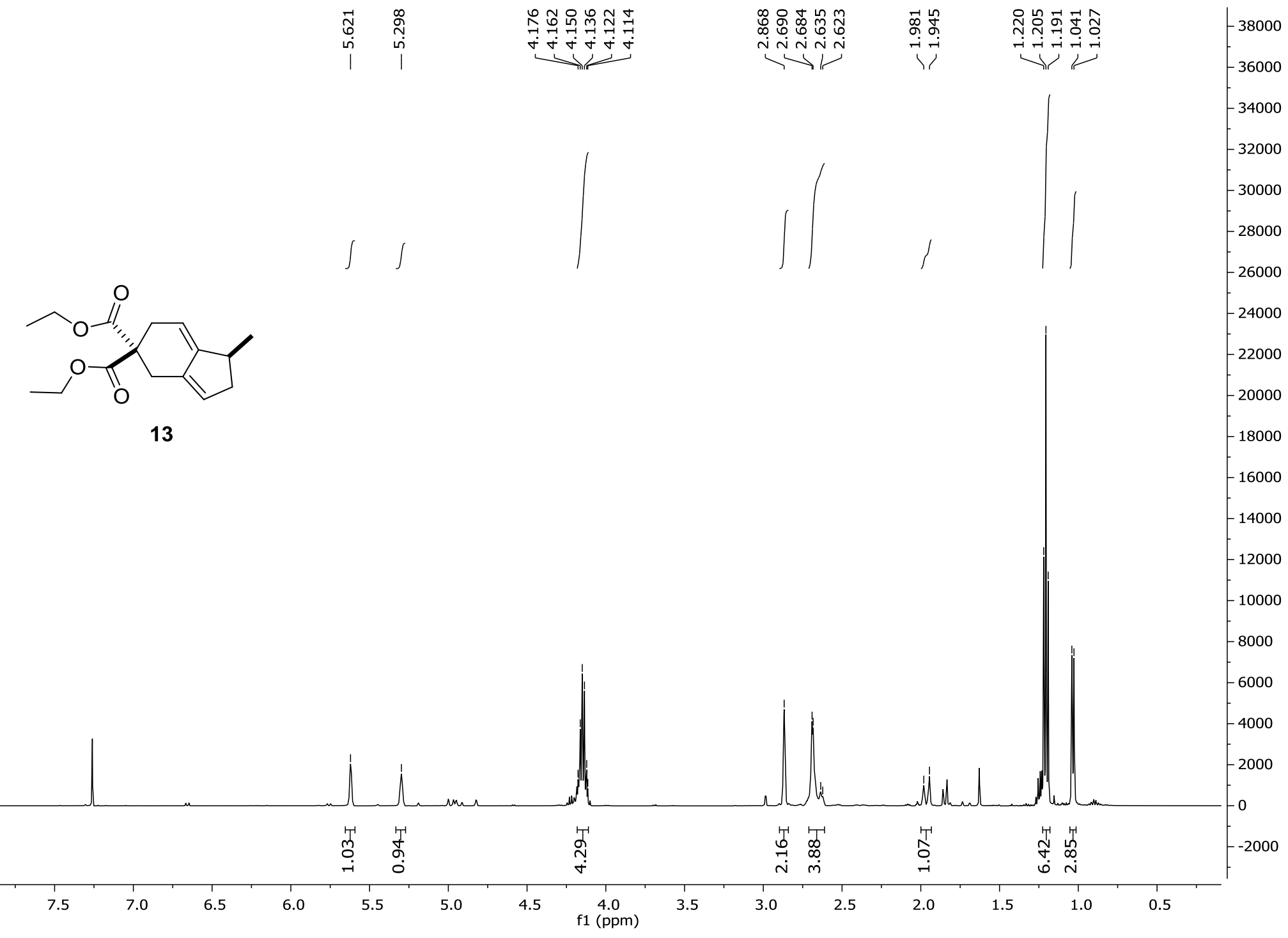


${ }^{13} \mathrm{C}$ NMR (125 MHz, $\left.\mathrm{CDCl}_{3}\right)$

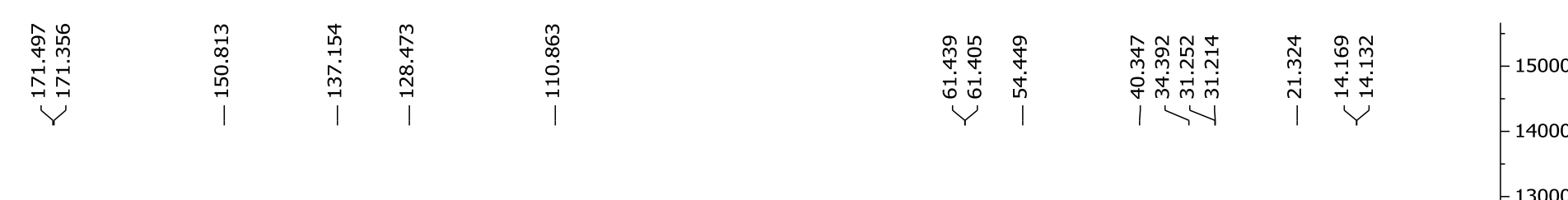

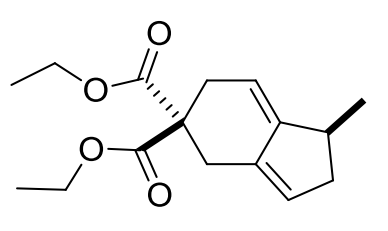

13

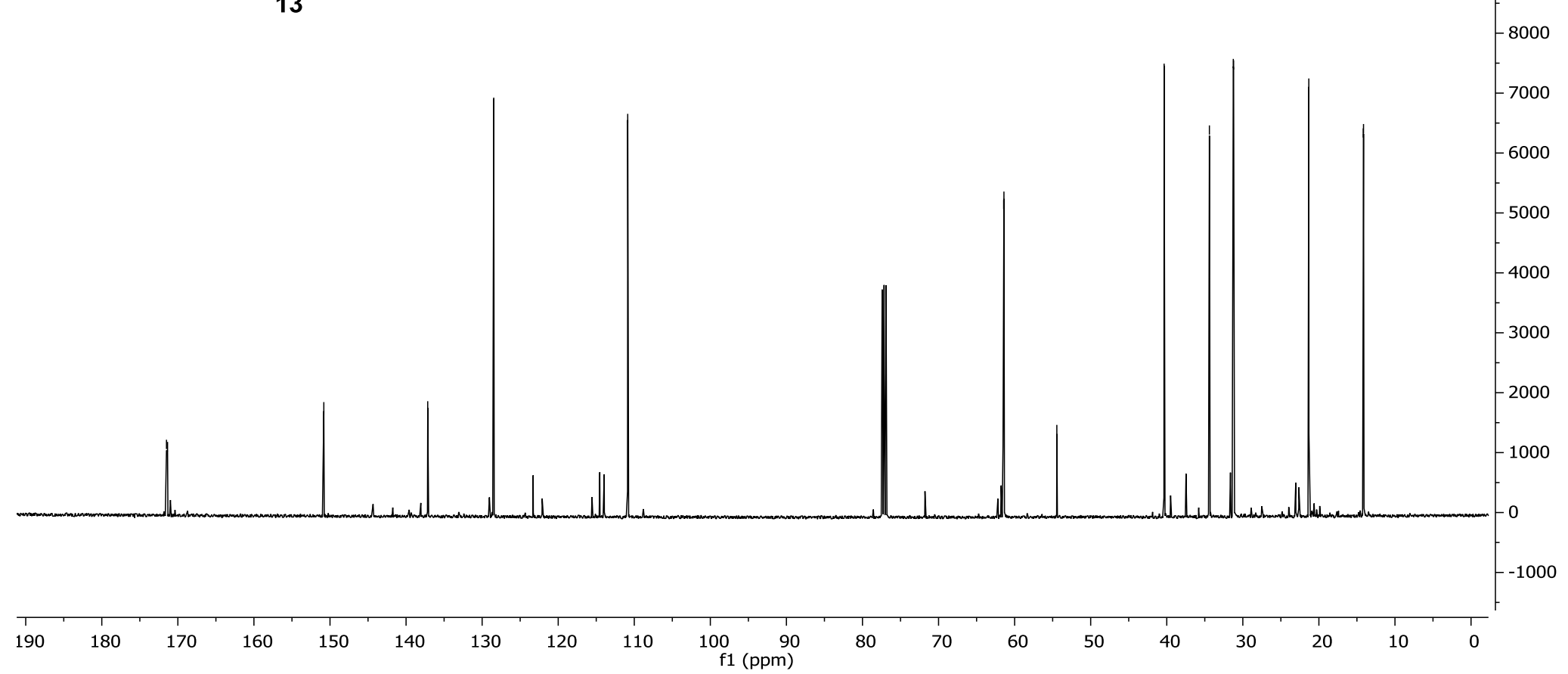


1H NMR (500 MHz, $\left.\mathrm{CDCl}_{3}\right)$

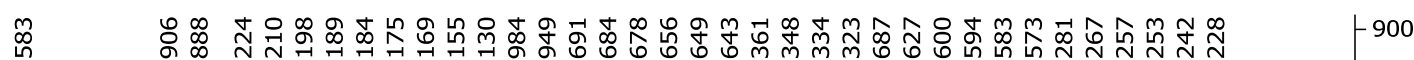

1) $\quad \underbrace{+1}$

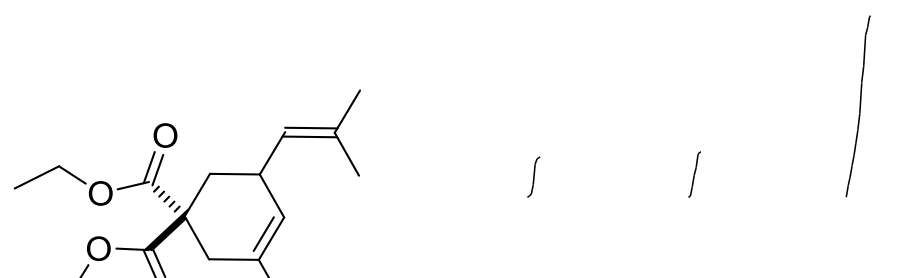

O Cl

15

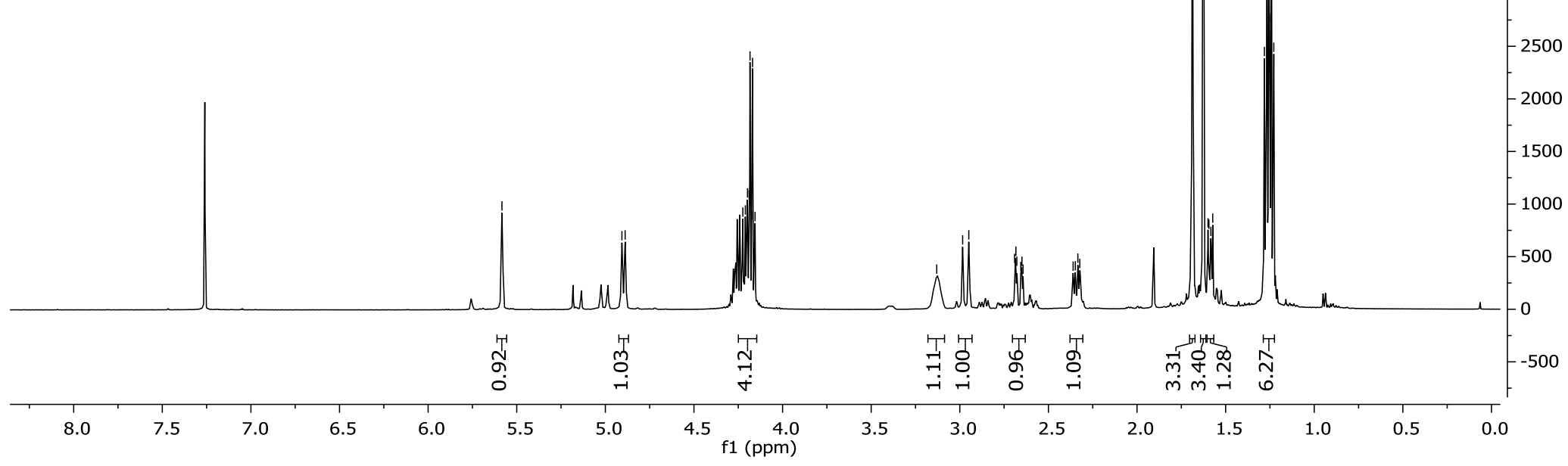




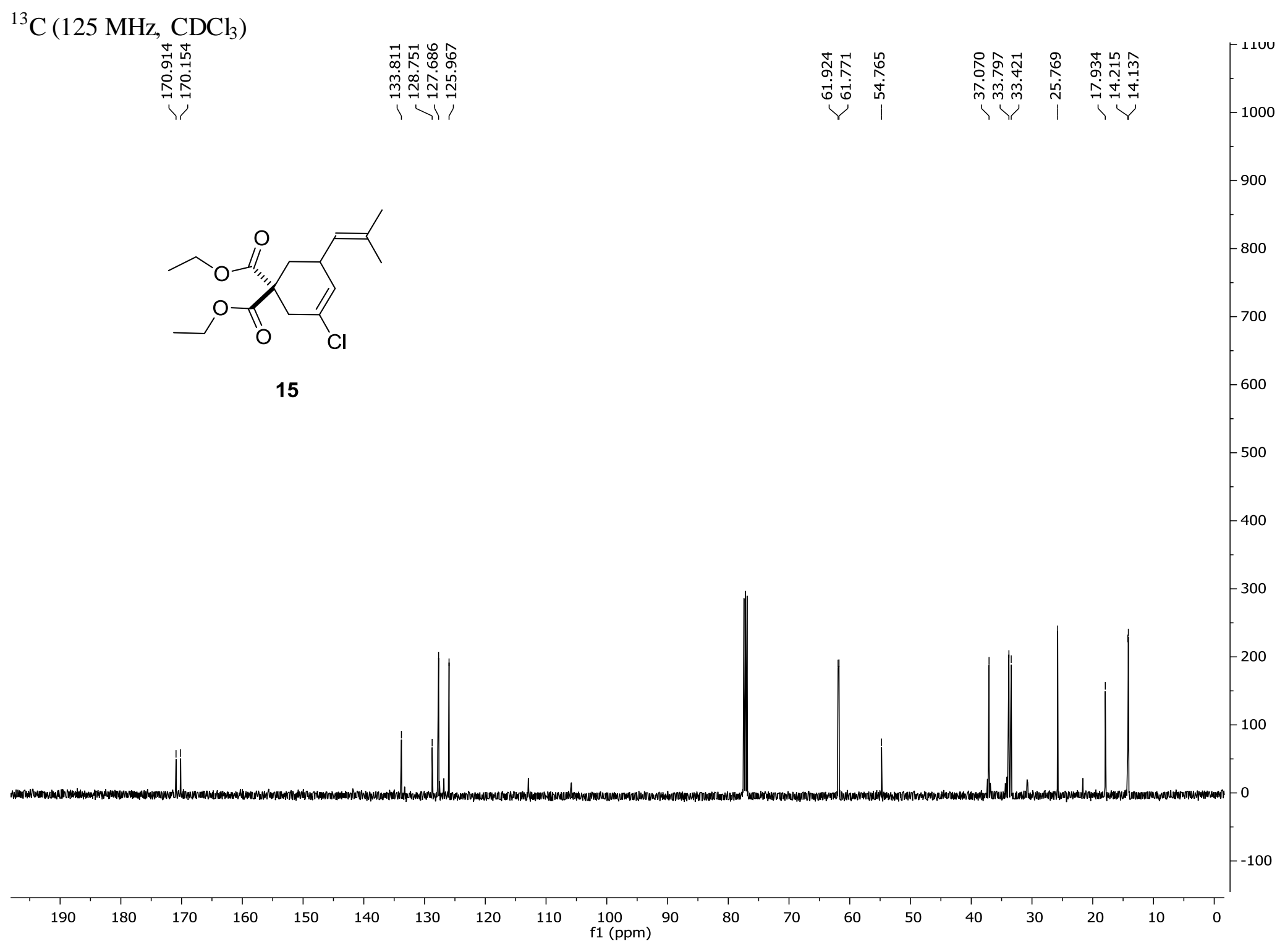




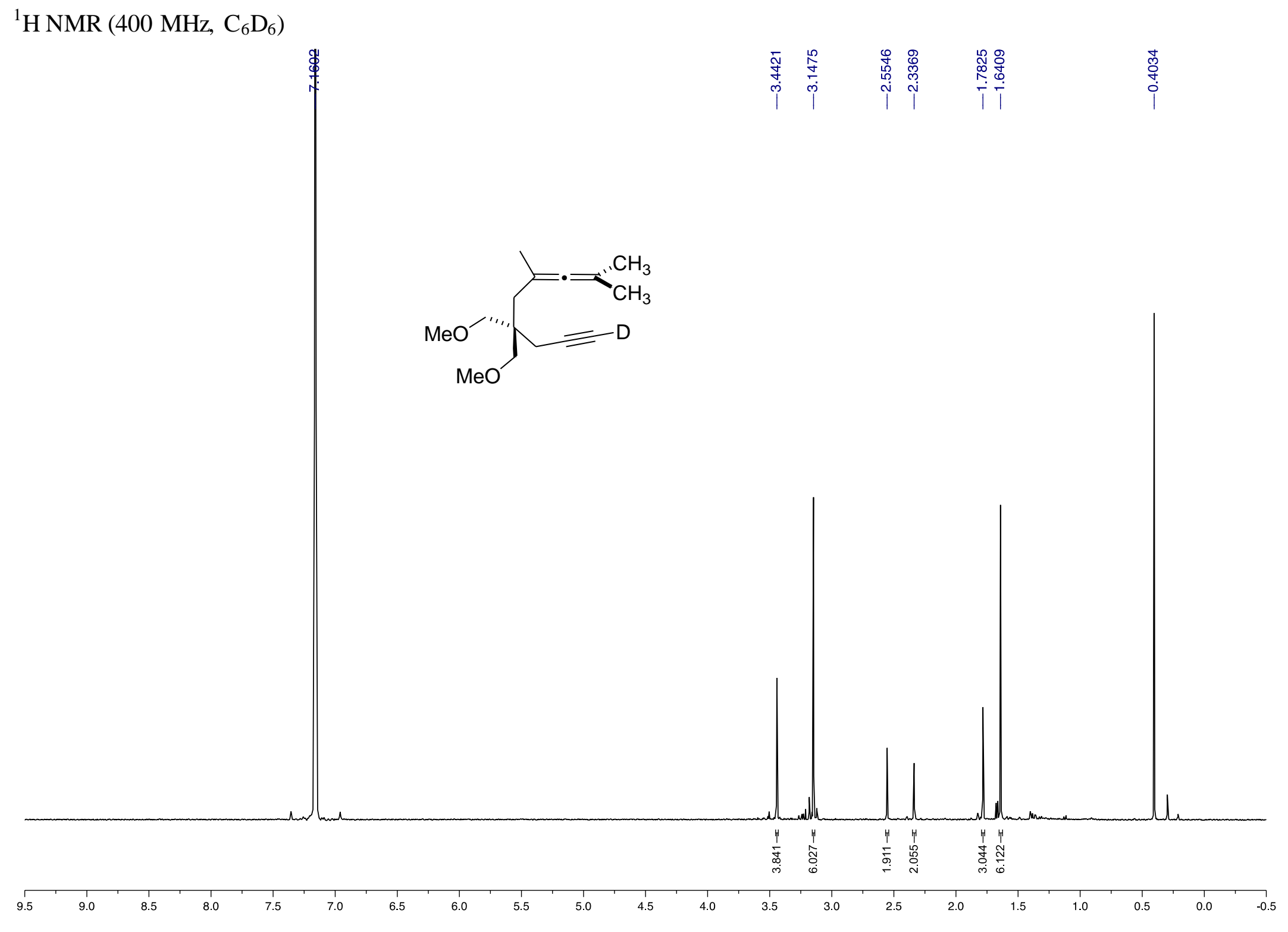




$$
-1
$$




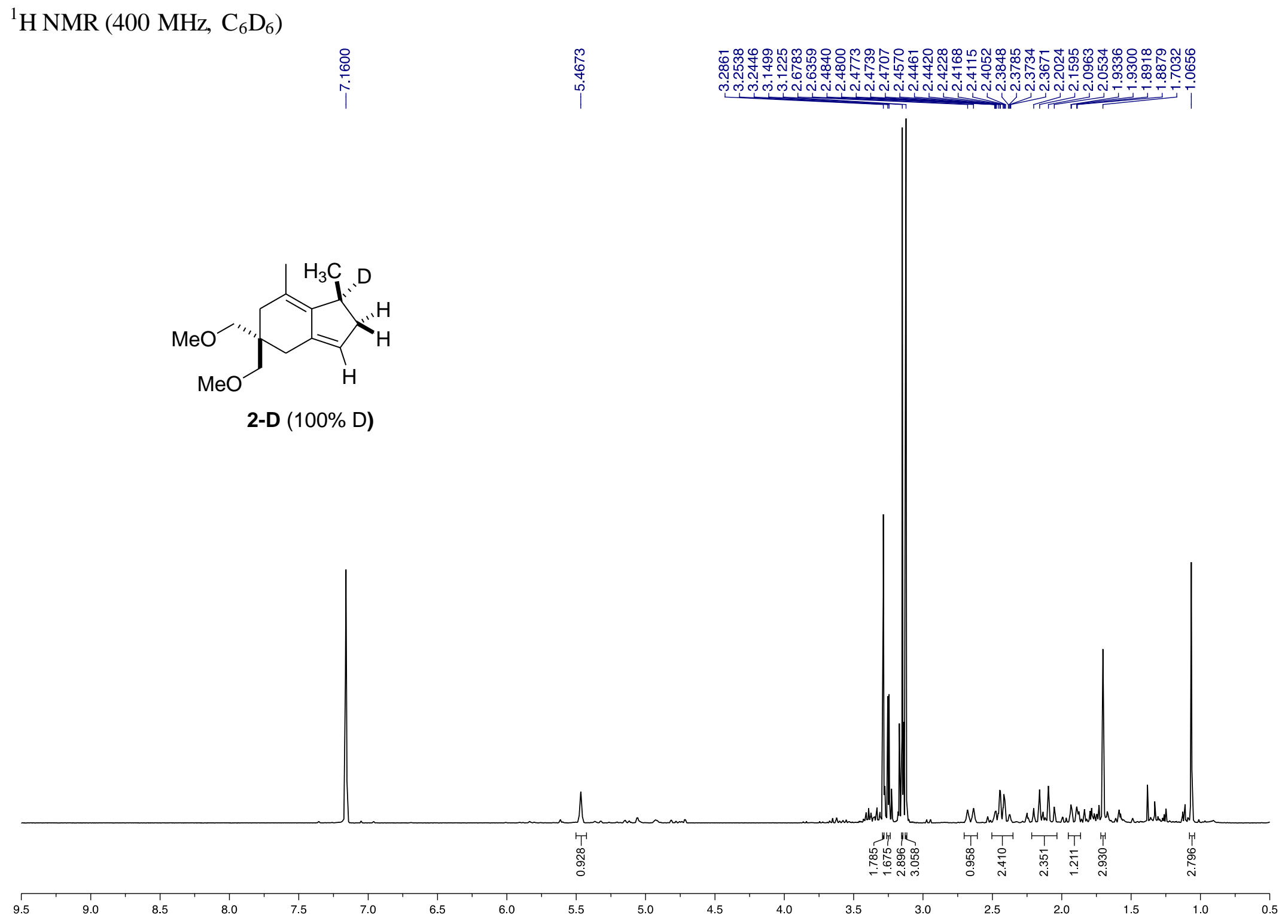


${ }^{13} \mathrm{C}$ NMR (100 MHz, $\mathrm{C}_{6} \mathrm{D}_{6}$ )
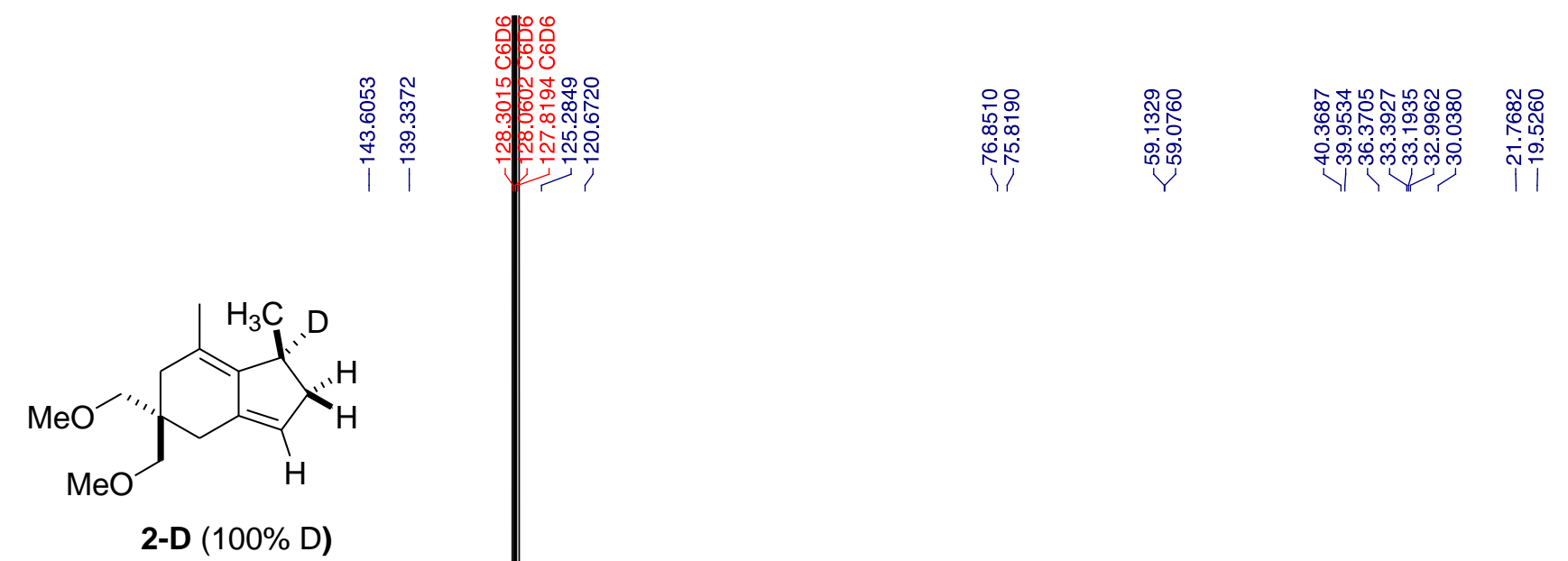

2-D (100\% D)

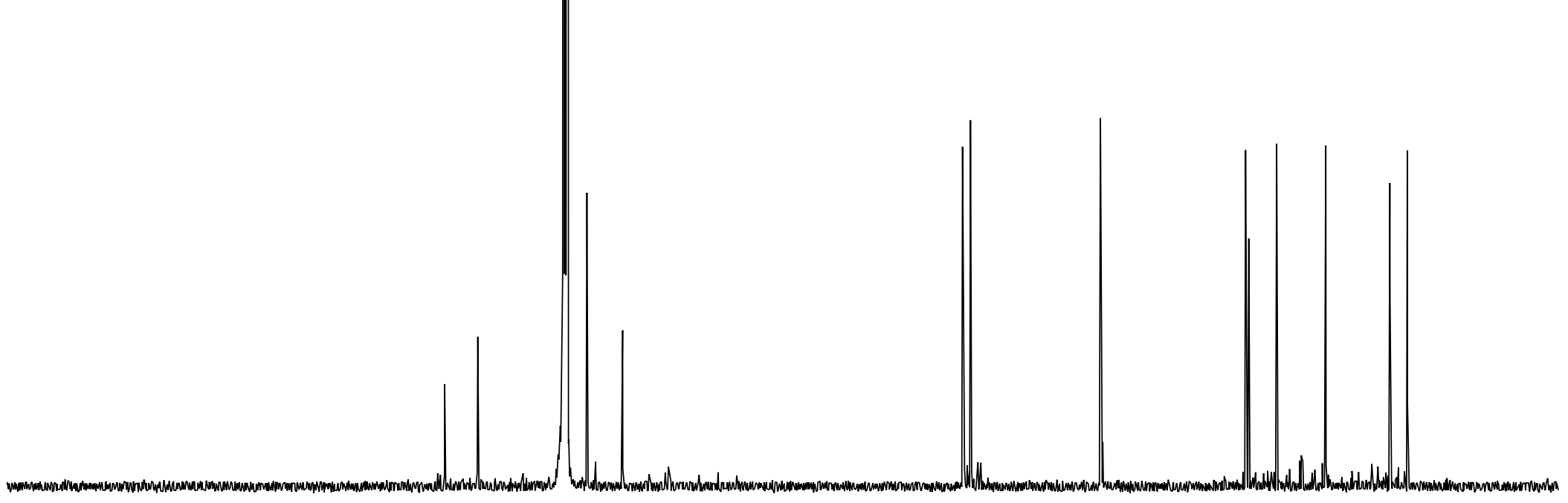




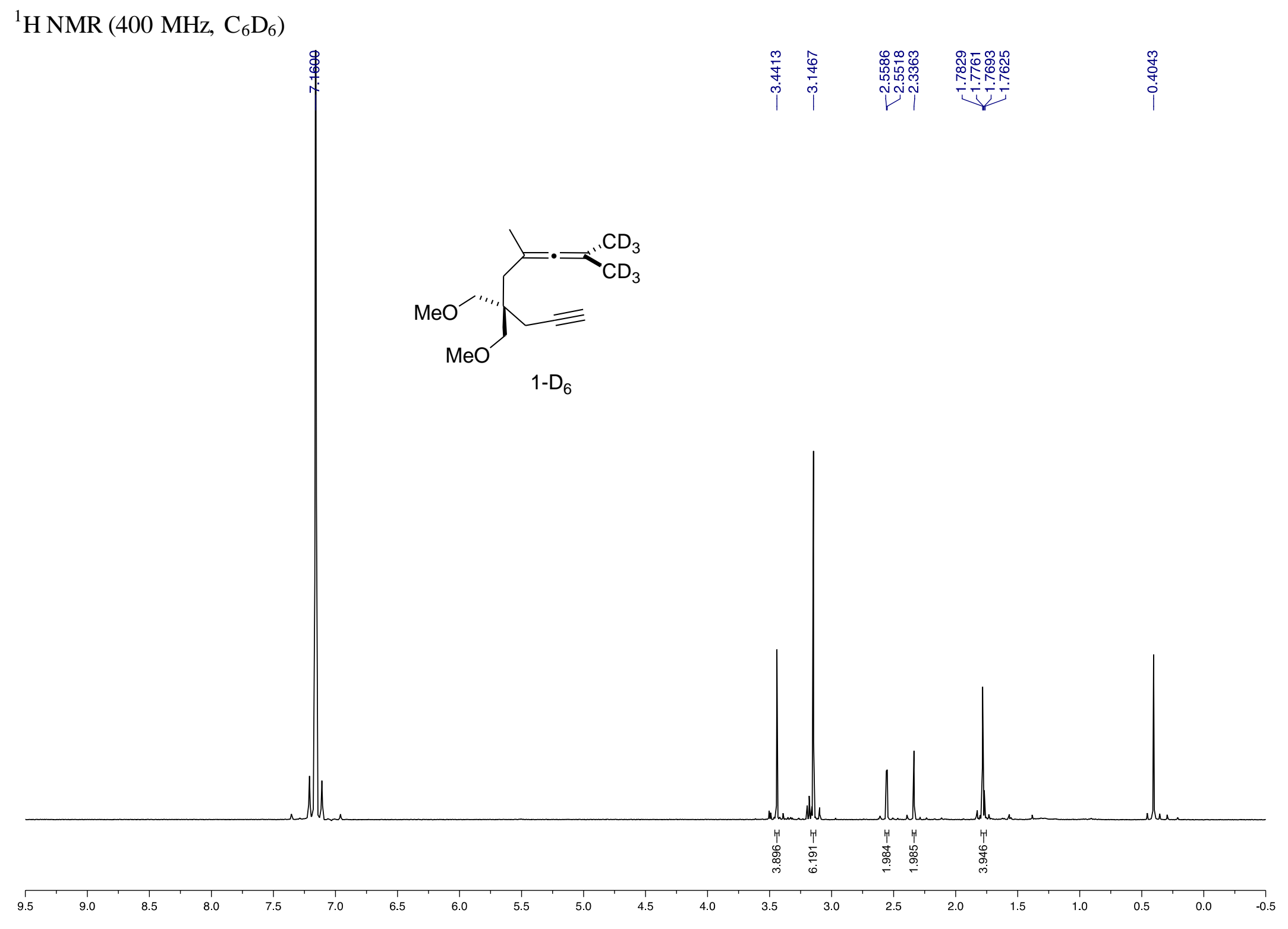


${ }^{13} \mathrm{C} \mathrm{NMR}\left(100 \mathrm{MHz}, \mathrm{CDCl}_{3}\right)$
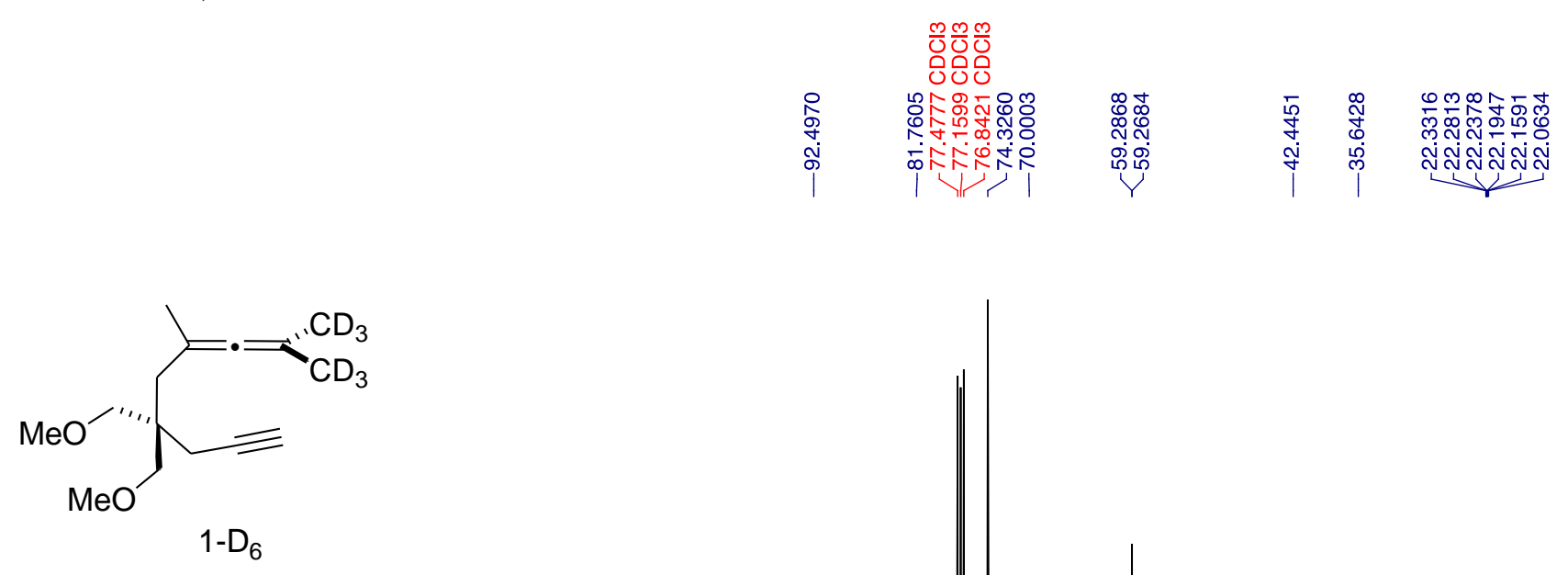

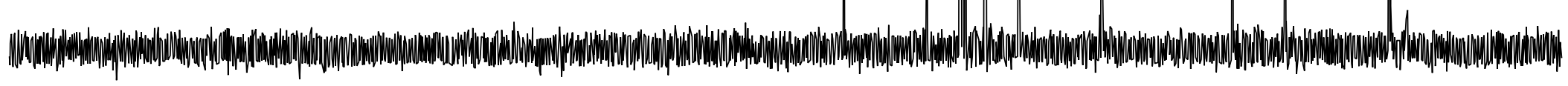


${ }^{1} \mathrm{H}$ NMR $\left(400 \mathrm{MHz}, \mathrm{C}_{6} \mathrm{D}_{6}\right)$

2- $D_{6}(100 \% D)$

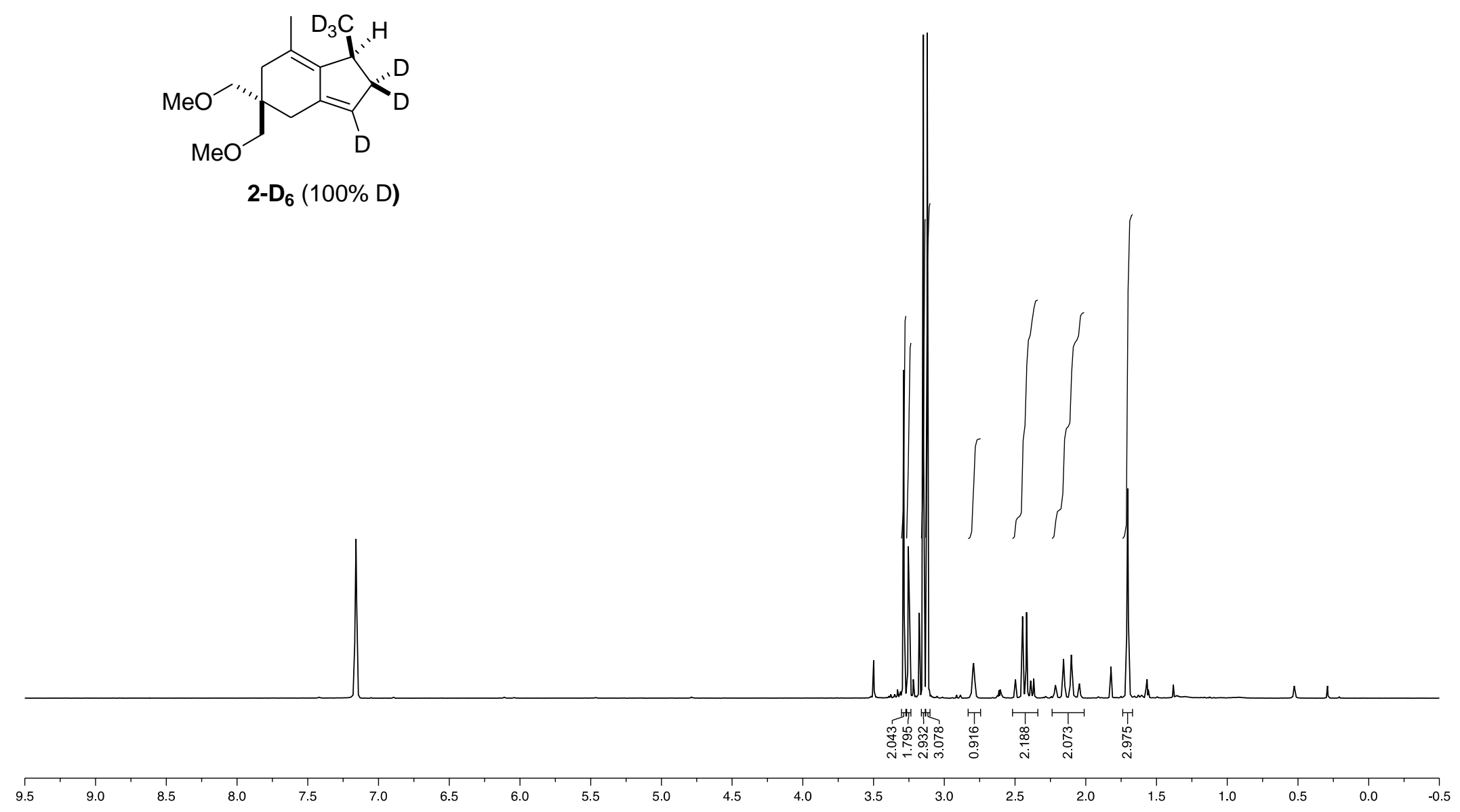

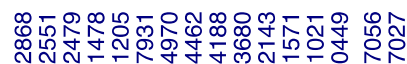

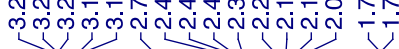


${ }^{13} \mathrm{C}$ NMR (100 MHz, $\left.\mathrm{C}_{6} \mathrm{D}_{6}\right)$

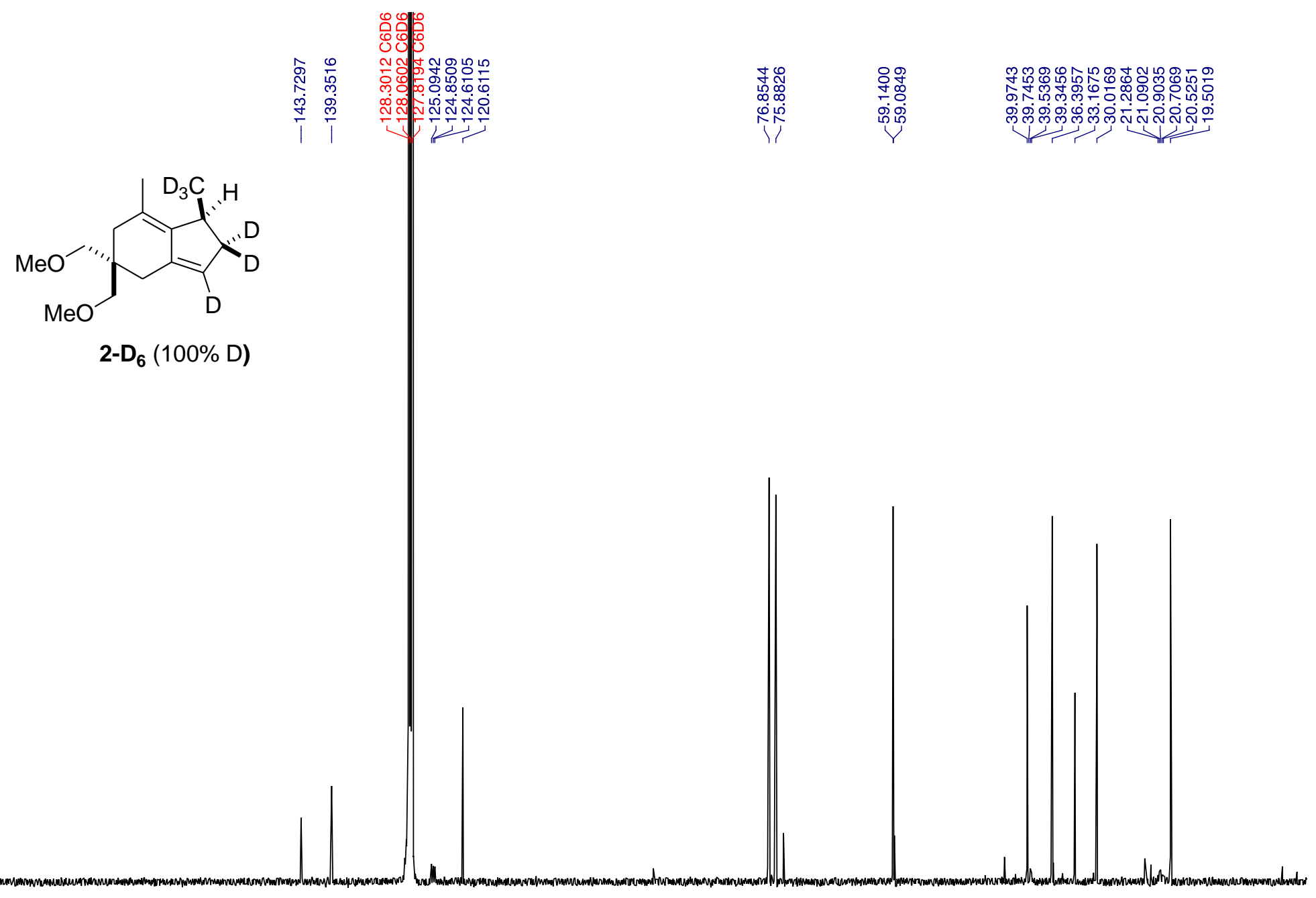

200

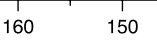

140

$120 \quad 110$
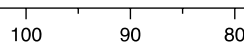

$70+60$

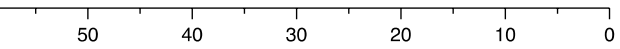




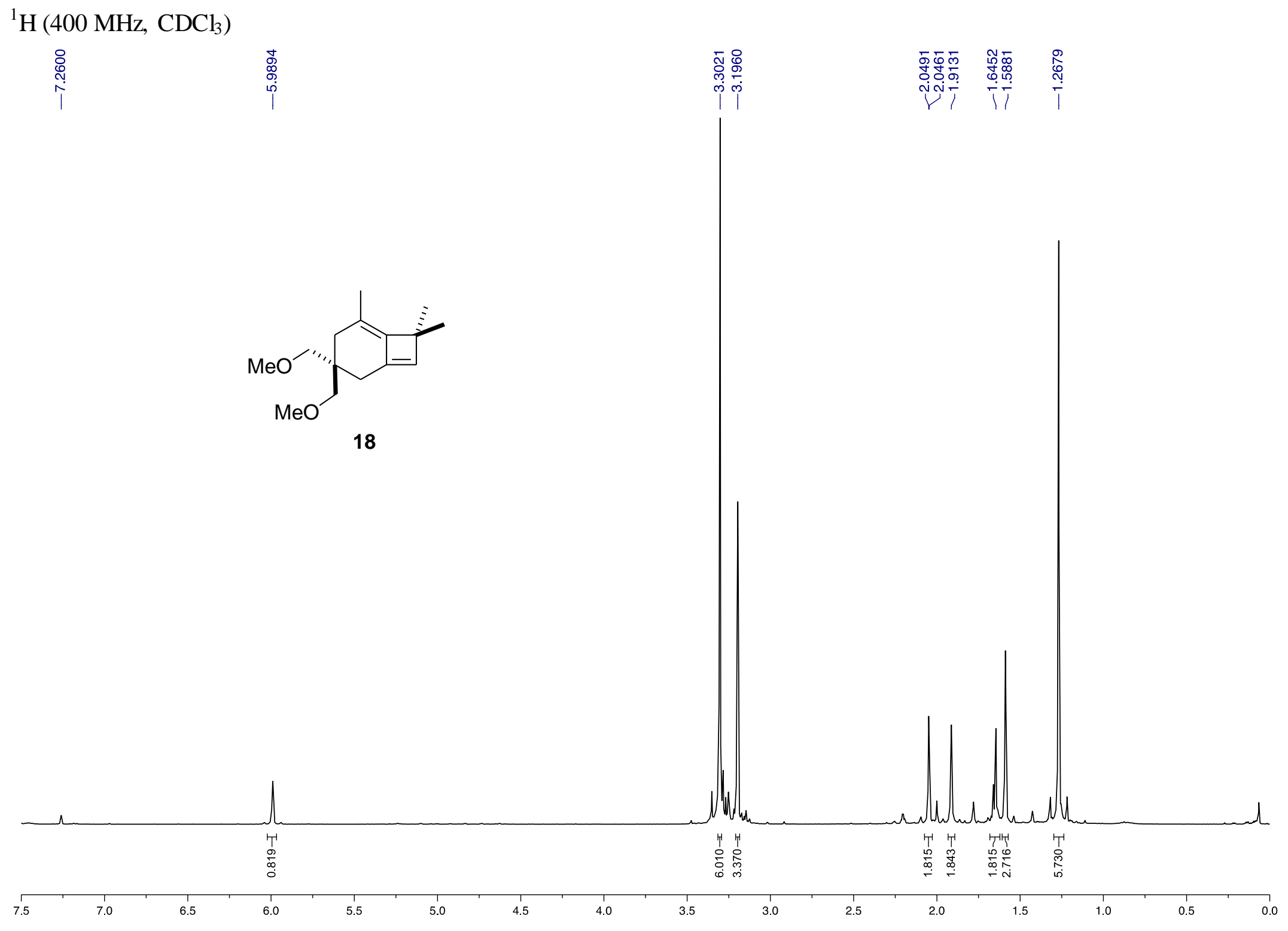


${ }^{13} \mathrm{C} \mathrm{NMR}\left(100 \mathrm{MHz}, \mathrm{CDCl}_{3}\right)$

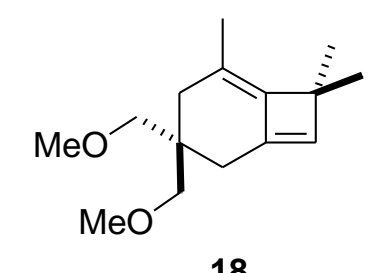

18

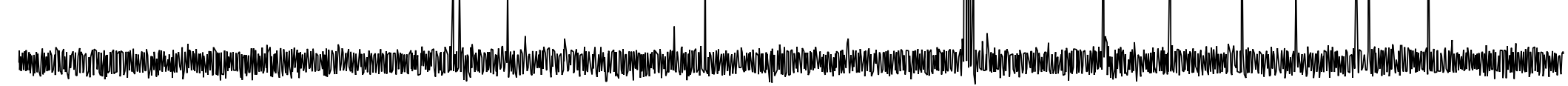


${ }^{1} \mathrm{H}$ NMR (400 MHz, $\mathrm{CDCl}_{3}$ )

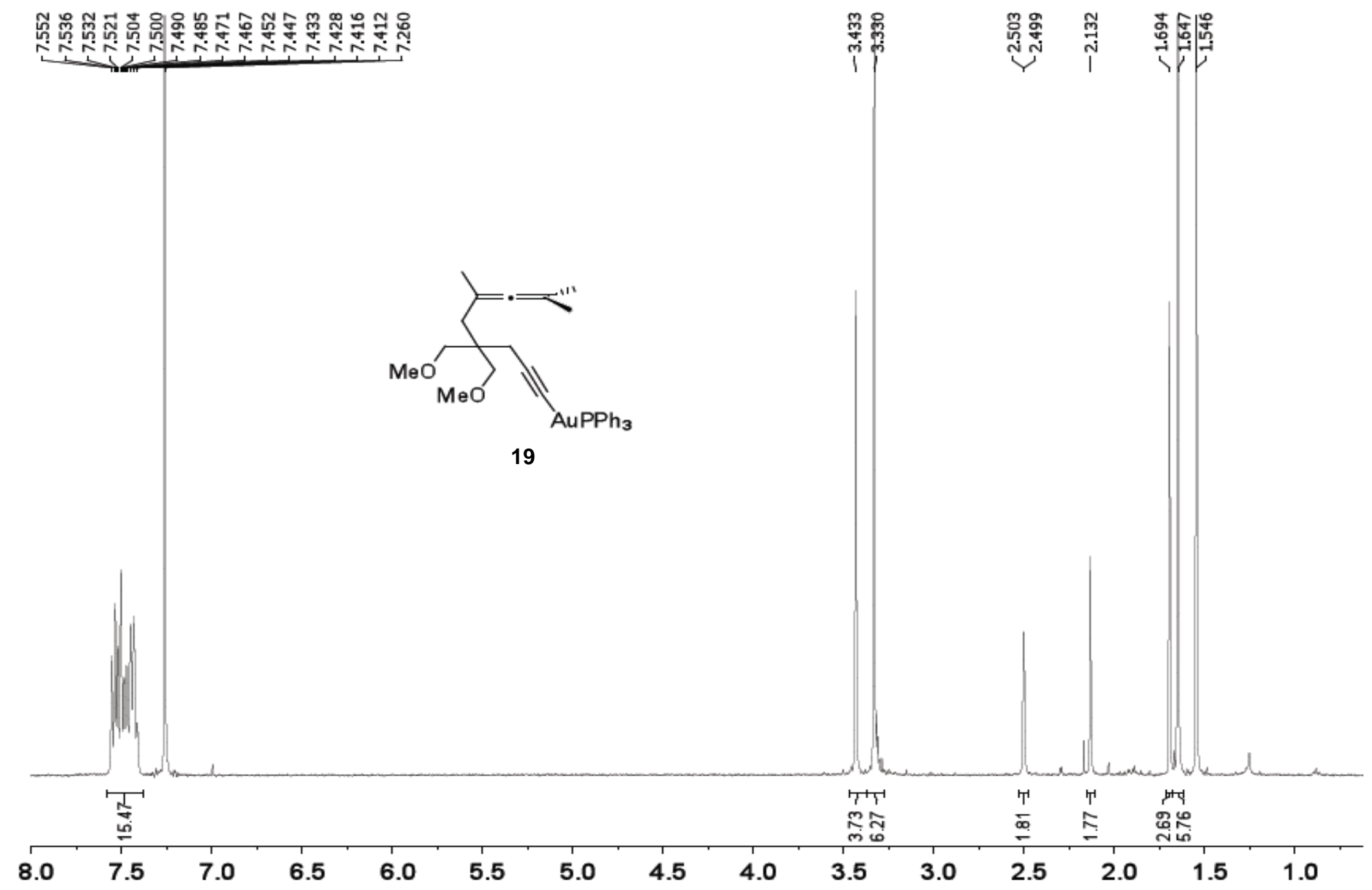




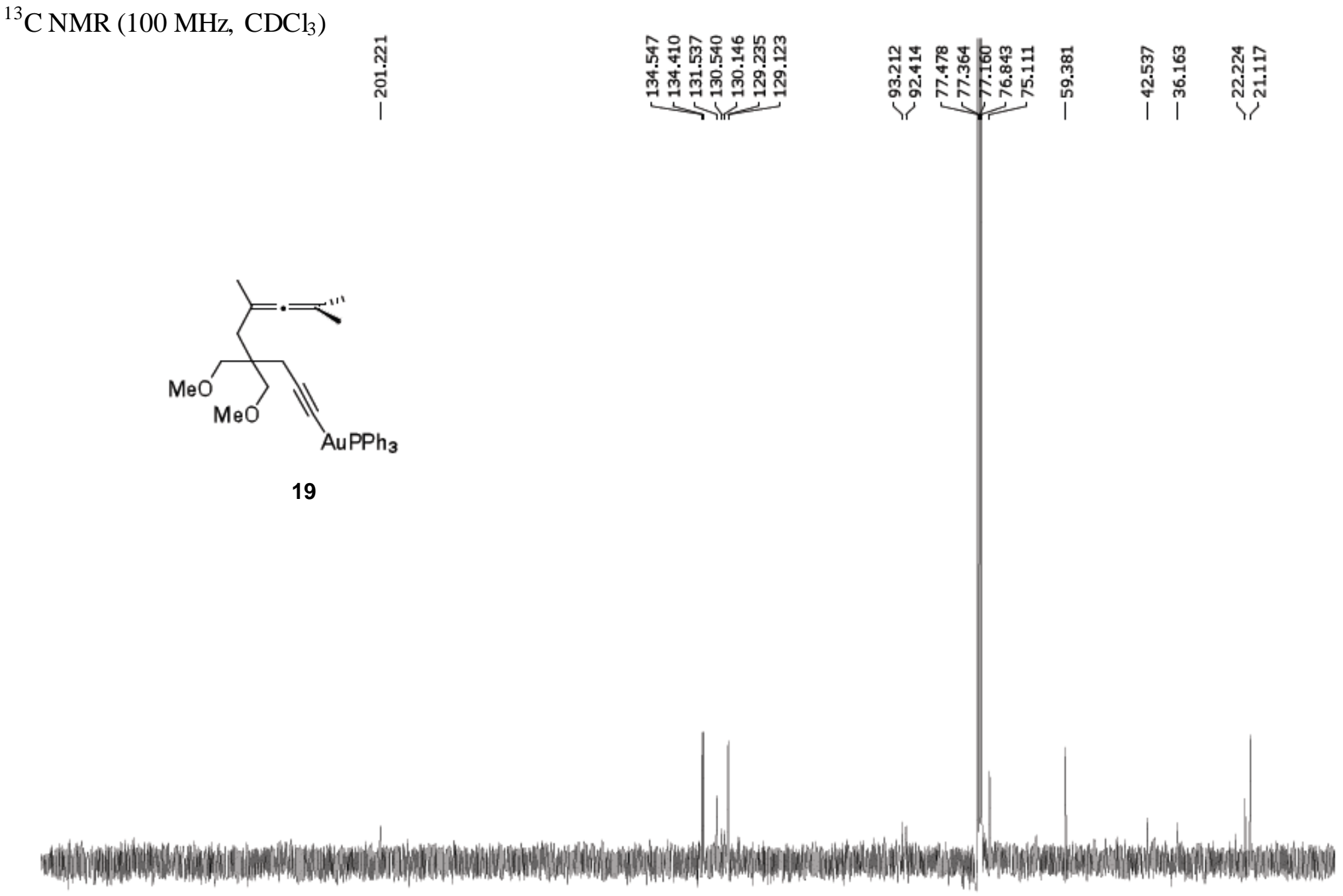

$\begin{array}{llllllllllllllll}260 & 240 & 220 & 200 & 180 & 160 & 140 & 120 & 100 & 80 & 60 & 40 & 20\end{array}$




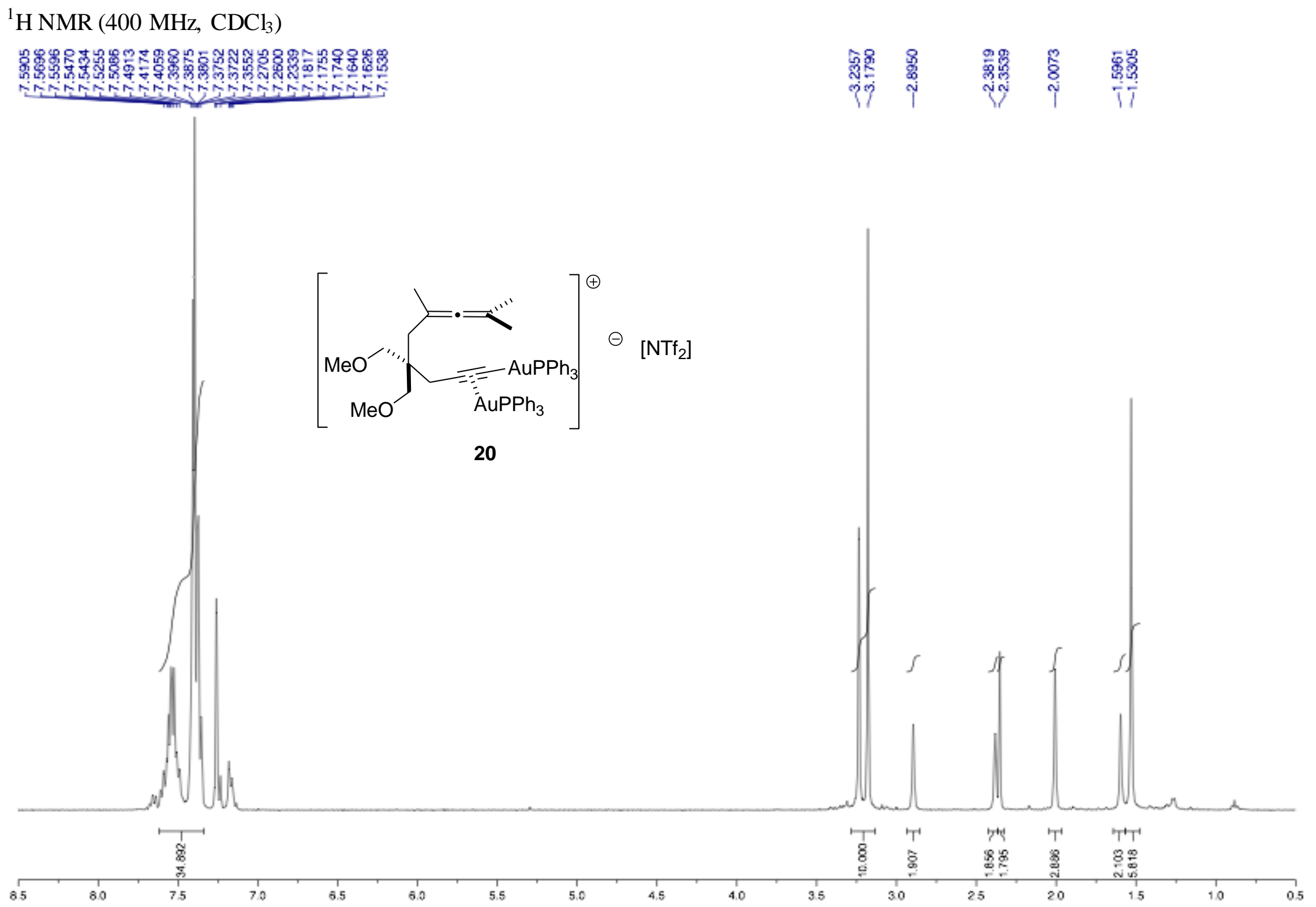


${ }^{13} \mathrm{C} \mathrm{NMR}\left(100 \mathrm{MHz}, \mathrm{CDCl}_{3}\right)$

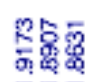

बู쪼

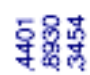

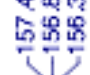

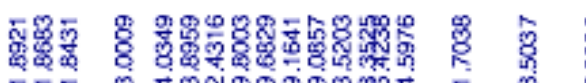

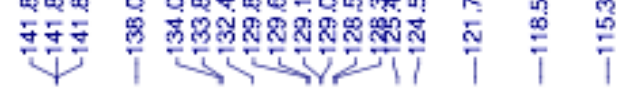

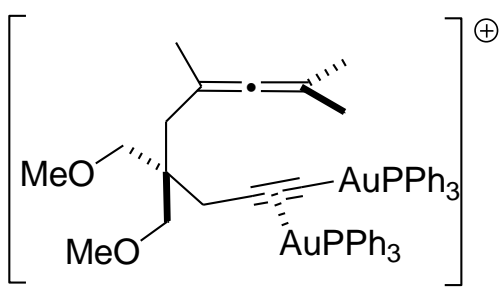

$\Theta\left[\mathrm{NTf}_{2}\right]$

20
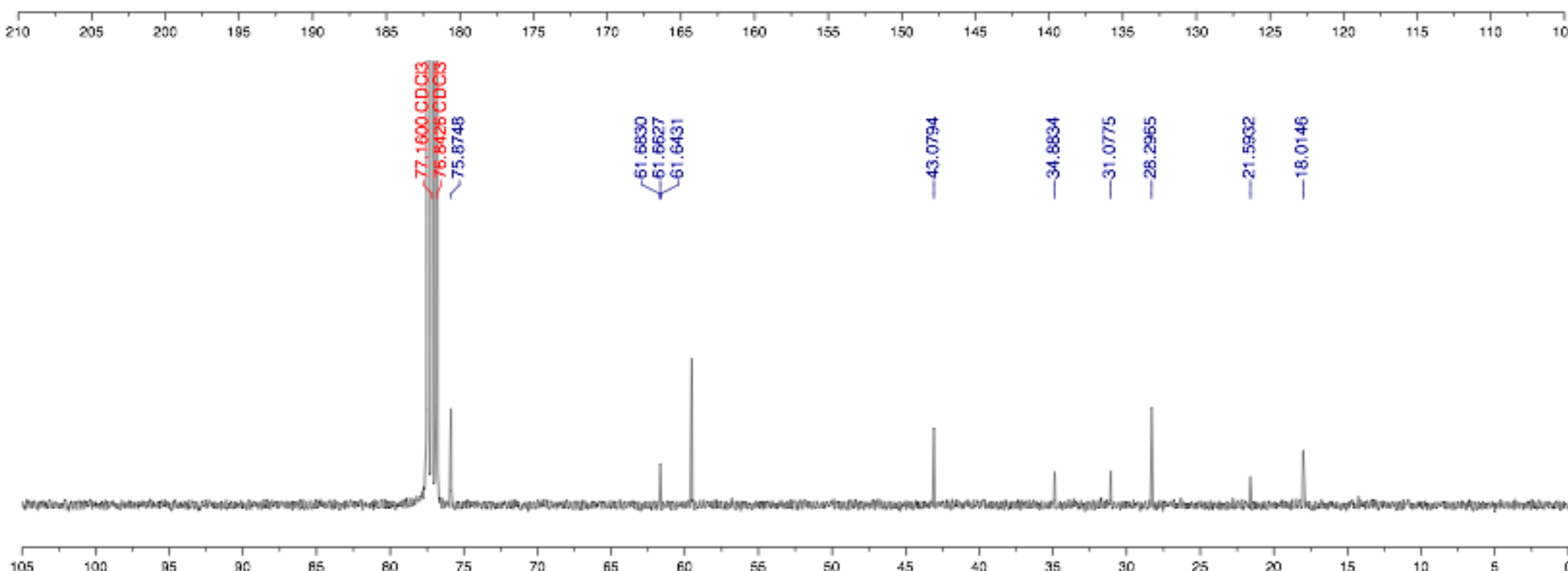
${ }^{31} \mathrm{P}$ NMR (162 MHz, $\left.\mathrm{CDCl}_{3}\right)$

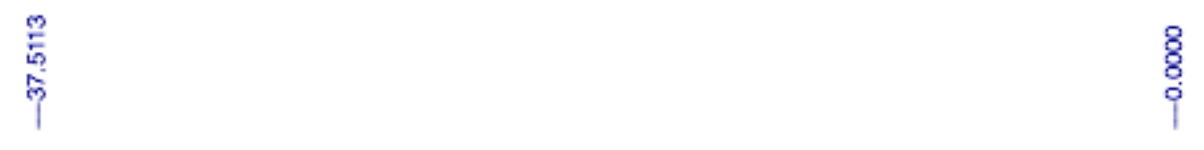

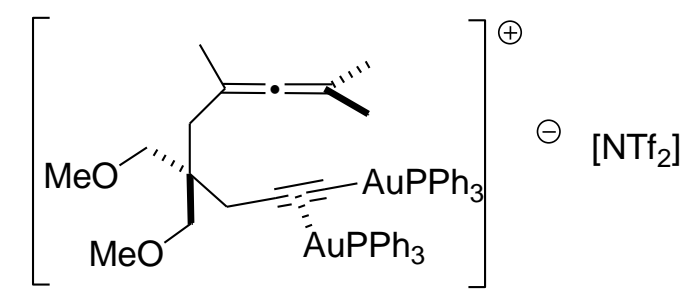

20

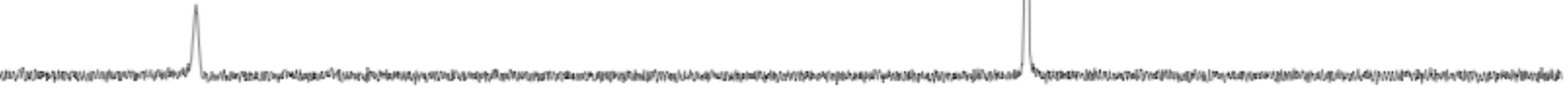

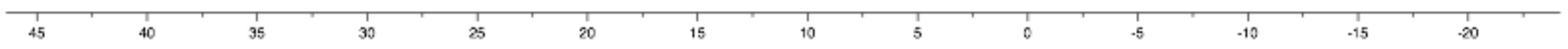

